Zentrum für Technologiefolgen-Abschätzung Centre d'évaluation des choix technologiques Centro per la valutazione delle scelte tecnologiche Centre for Technology Assessment
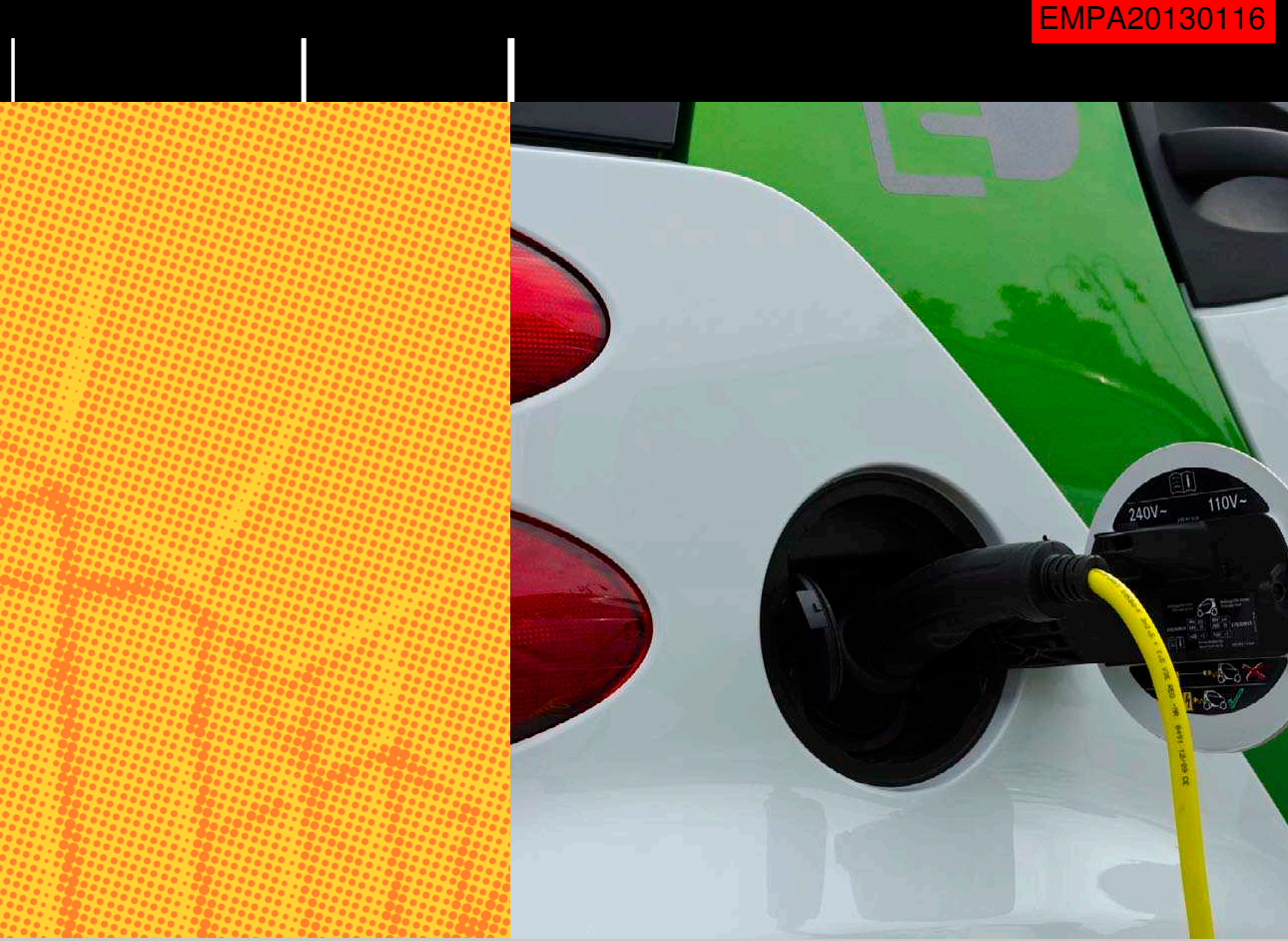

Peter de Haan, Rainer Zab

\title{
Chancen und Risiken der Elektromobilität in der Schweiz
}



Zentrum für Technologiefolgen-Abschätzung Centre d'évaluation des choix technologiques Centro per la valutazione delle scelte tecnologiche Centre for Technology Assessment

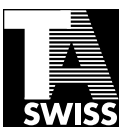

TA-SWISS 59/2013

Peter de Haan, Rainer Zab mit Beiträgen von Hans-Jörg Althaus, Katrin Bernath, Frank Bruns, Denise Fussen, Marcel Gauch, Patrick Wäger, Rolf Widmer

\section{Chancen und Risiken der Elektromobilität in der Schweiz}


Bibliografische Information Der Deutschen Nationalbibliothek Die Deutsche Nationalbibliothek verzeichnet diese Publikation in der Deutschen Nationalbibliografie; detaillierte bibliografische Daten sind im Internet über http://dnb.d-nb.de abrufbar.

Dieses Werk einschliesslich aller seiner Teile ist urheberrechtlich geschützt. Jede Verwertung ausserhalb der engen Grenzen des Urheberrechtsgesetzes ist ohne Zustimmung des Verlages unzulässig und strafbar. Das gilt insbesondere für Vervielfältigungen, Übersetzungen, Mikroverfilmungen und die Einspeicherung und Verarbeitung in elektronischen Systemen.

Die Studie «Chancen und Risiken der Elektromobilität in der Schweiz» wurde unterstützt vom Bundesamt für Energie BFE, vom Bundesamt für Strassen ASTRA sowie vom Bundesamt für Umwelt BAFU.

This work is licensed under a creative commons license<smiles>C1C[C@H]2[C@H]1[C@@H]1CC[C@H]21</smiles>

\section{(C) 2013 vdf Hochschulverlag AG an der ETH Zürich}

ISBN 978-3-7281-3487-5 (Printausgabe)

Download open access:

ISBN 978-3-7281-3488-2 / DOI 10.3218/3488-2

www.vdf.ethz.ch

verlag@vdf.ethz.ch 


\section{Inhalt}

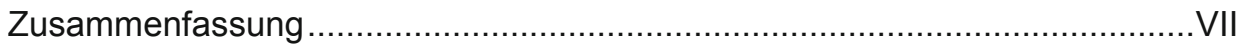

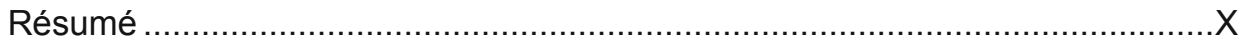

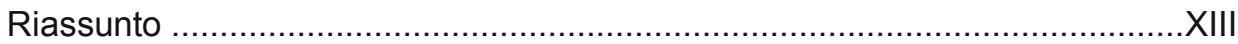

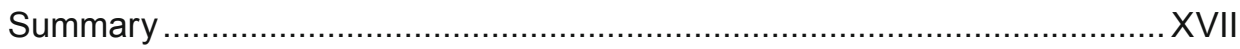

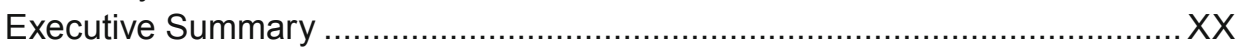

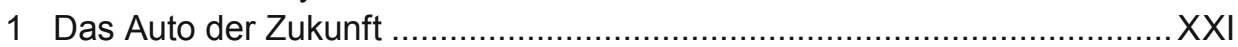

2 Elektromobilität in der Schweiz ...........................................................

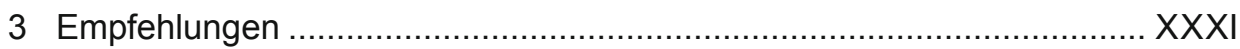

Aufbau des vorliegenden Berichts ........................................................XXII

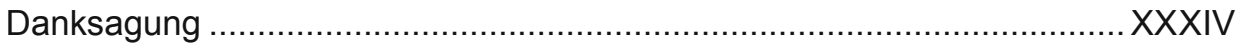

1 Bedeutung von Elektrofahrzeugen im Kontext unserer Mobilität und des Energiesystems ............................................................. 1

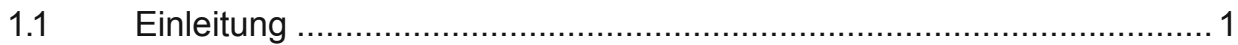

1.1.1 Warum eine TA-SWISS-Studie zur Elektromobilität? ......................... 1

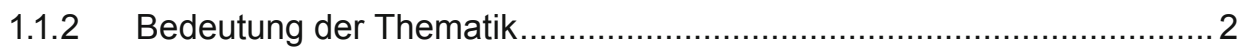

1.1.3 Systemabgrenzung ................................................................ 3

1.1.4 Ziele der vorliegenden Technikfolgenabschätzung zur Elektromobilität: sechs Leitfragen ............................................................. 3

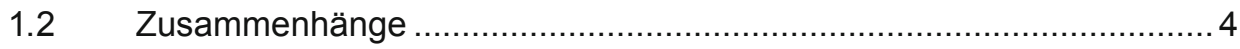

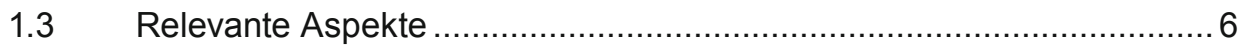

$1.4 \quad$ Allgemeine Definitionen ............................................................

1.5 Methodisches Vorgehen für die quantitative Berechnung der Chancen und Risiken................................................................ 9

$2 \quad$ Definition der drei Szenarien........................................................ 11

2.1 Systemgrenze und zu definierende Parameter................................ 11

$2.2 \quad$ Eckpunkte der Szenarien ............................................................ 12

2.3 Entwicklung von PKW-Politik, -Angebot und -Nachfrage ................... 14

2.4 Marktanteile der Elektromobile bis 2035 und 2050 .......................... 17

2.5 Produktionskapazität von Elektromobilen bis $2025 \ldots \ldots \ldots \ldots \ldots \ldots \ldots \ldots . . . .20$

2.6 Mikrosimulation der Elektromobil-Marktanteile bis 2035 …................23

2.7 Modellierung der Elektromobil-Anteile 2035 bis $2050 \ldots \ldots \ldots \ldots \ldots \ldots \ldots . . . .26$

2.8 Entwicklung von Bevölkerung, Motorisierungsgrad, Fahrleistung und Fahrzeugflotte ............................................................... 27

$2.9 \quad$ Entwicklung des Energiesystems (inkl. Strommix) ......................... 28 
3 Zukünftige Entwicklung der Fahrzeugkomponenten .........................29

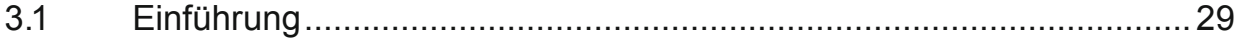

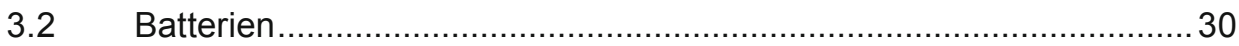

3.2.1 Gegenwärtiger Stand der Technologie ........................................ 31

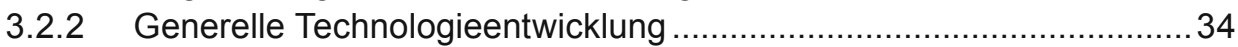

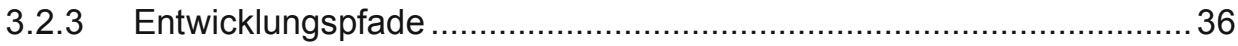

3.2.4 Annahmen der Studie für die Batterieentwicklung ............................ 38

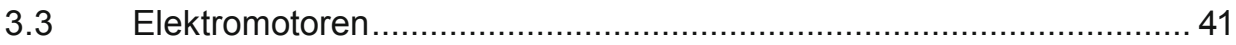

3.3.1 Gegenwärtiger Stand der Technologie .......................................... 41

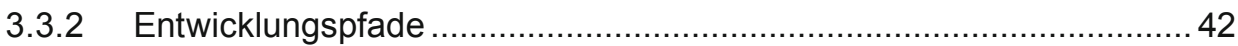

3.3.3 Annahmen der Studie für die Elektromotor-Entwicklung .................... 44

3.4 Verbrennungsmotoren (ICE-Motoren) ...........................................45

3.4.1 Gegenwärtiger Stand der Technologie .........................................46

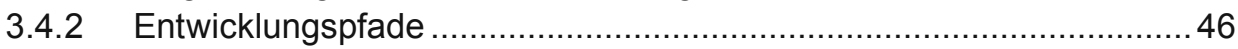

3.4.3 Annahmen der Studie für die ICE-Motorenentwicklung..................... 47

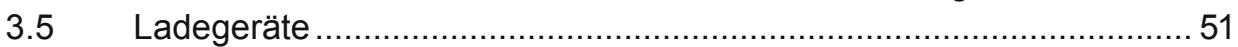

3.5.1 Gegenwärtiger Stand der Technologie …...................................... 51

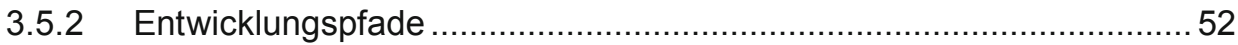

3.5.3 Annahme der Studie für die Entwicklung der Ladegeräte ...................54

3.6 Sicherheit und Komfort ........................................................... 54

3.6.1 Gegenwärtiger Stand der Technologie ..........................................54

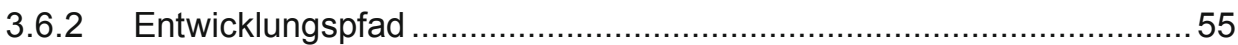

3.6.3 Annahmen der Studie für die Entwicklung von Sicherheit

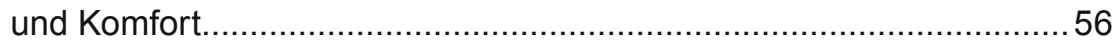

$4 \quad$ Zukünftige Entwicklung der Fahrzeuge ............................................ 59

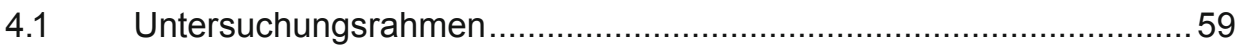

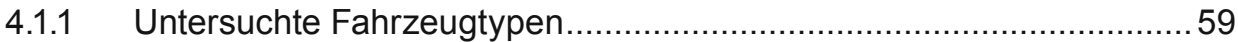

4.1.2 Gegenwärtiger Stand der Technologie ..........................................60

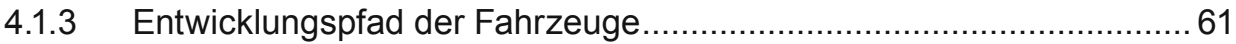

4.1.4 Annahmen der Studie für die Entwicklung der Fahrzeugparameter .... 61

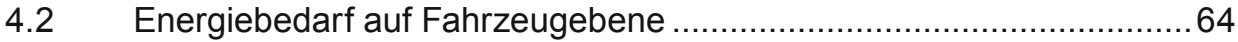

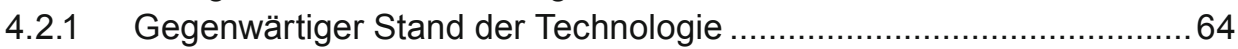

4.2.2 Methodik zur Bestimmung des Fahrzeugenergieverbrauchs..............66

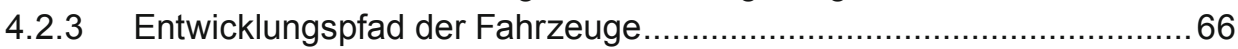

4.2.4 Annahmen der Studie für die Entwicklung des Fahrzeug-

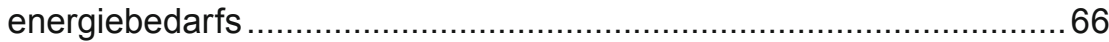

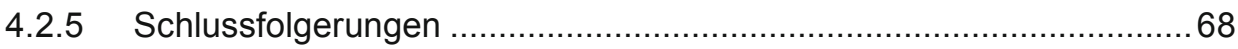

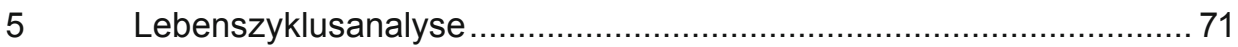

5.1 Ziel und Rahmen der Lebenszyklusanalyse .................................. 71 


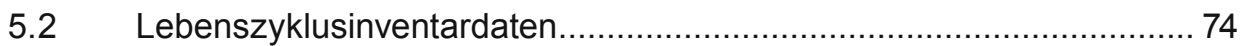

5.3 Lebenszyklusinventardaten zur Energiebereitstellung...................... 77

$5.4 \quad$ Resultate auf Stufe «Fahrzeug»................................................... 80

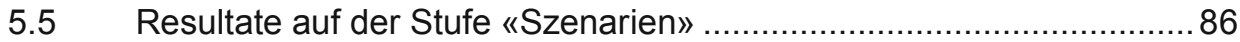

5.6 Unsicherheiten der Lebenszyklusanalye .......................................90

5.6.1 Sensitivitätsanalyse der Annahmen zu den Fahrzeug-

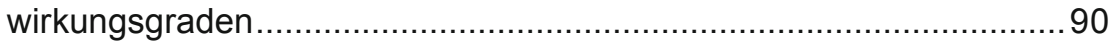

5.6.2 Sensitivitätsanalyse zur Stromerzeugung.................................... 91

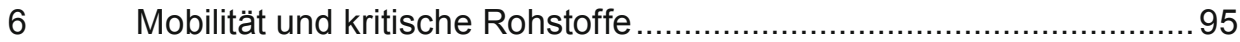

6.1 Kritikalität metallischer Rohstoffe ...................................................95

6.2 Kritische metallische Rohstoffe in den untersuchten

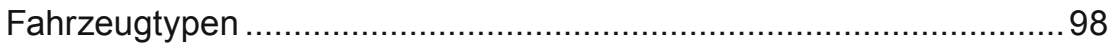

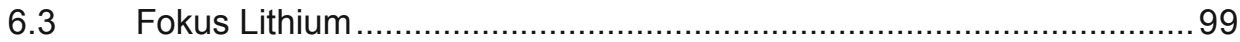

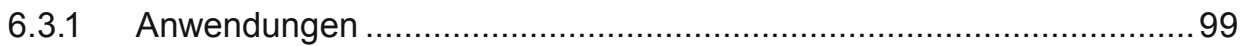

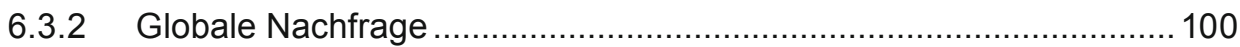

6.3.3 Nachfrage durch Neuwagen in der Schweiz.................................. 101

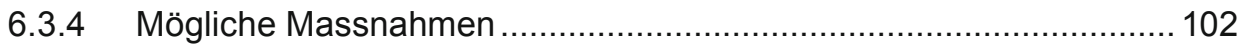

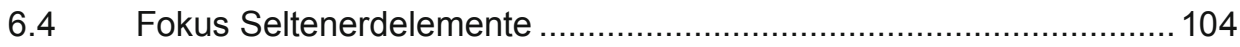

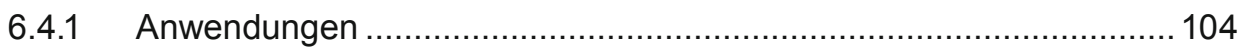

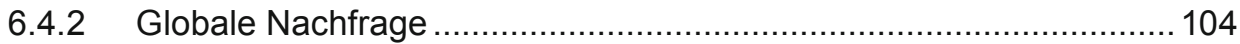

6.4.3 Nachfrage durch Neuwagen in der Schweiz ................................. 106

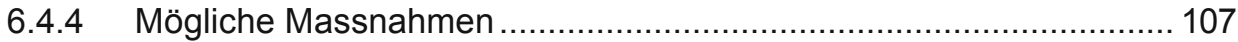

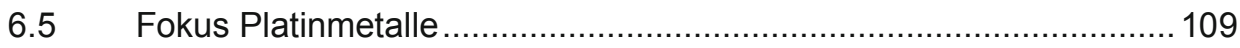

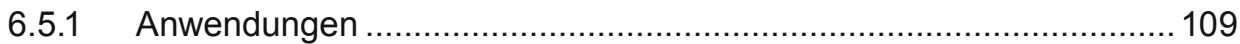

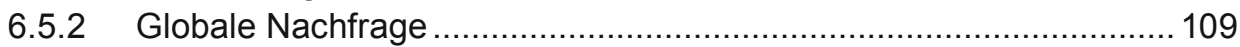

6.5.3 Nachfrage durch Neuwagen in der Schweiz................................. 110

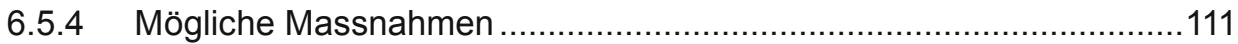

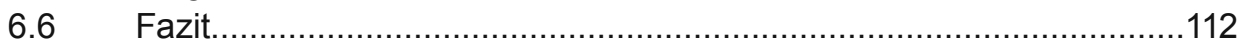

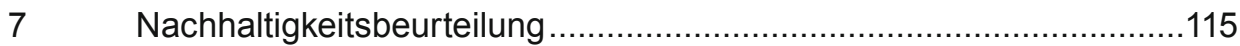

7.1 Wirtschaftliche Relevanz der Automobilbranche .............................115

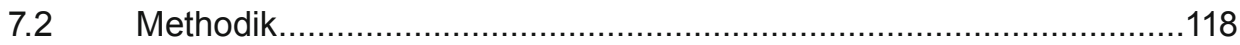

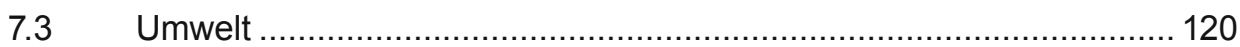

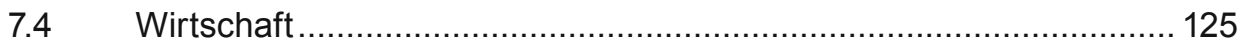

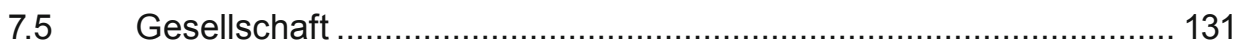

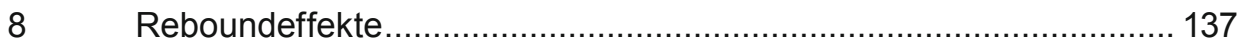

8.1 Treiber für Reboundeffekte ...................................................... 137

8.2 Können effiziente Fahrzeuge Reboundeffekte induzieren? ............... 138

8.3 Massnahmen zur Reduktion von Reboundeffekten ........................ 141

$9 \quad$ Gesamtbeurteilung, Fazit und Empfehlung................................... 143 
9.1 Resultate für alle Szenarien ........................................................ 143

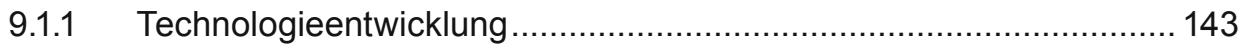

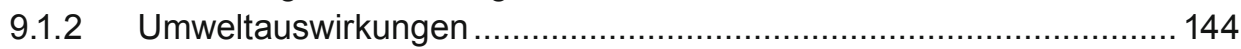

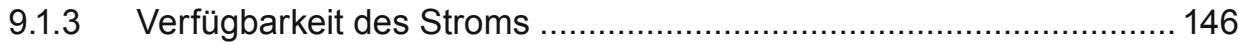

9.1.4 Rohstoffbeanspruchung...................................................... 147

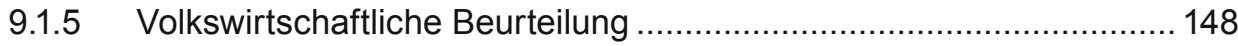

$9.2 \quad$ Gesamtbeurteilung und Fazit......................................................... 150

9.3 Diskussion und Empfehlungen zu den Leitfragen........................... 152

9.4 Mögliche Massnahmen aus der Perspektive der Stakeholder........... 160

Anhänge

A1 Literaturverzeichnis ............................................................ 169

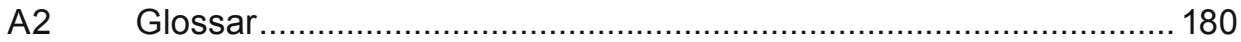

A3 Potenzial für Verlagerung von MIV-Fahrleistung hin zu Kleinst-EV (und Bahn) ................................................................................ 183

A4 Detaillierte Grundlagen zu Kapitel 2 (Marktpenetration von Elektro-

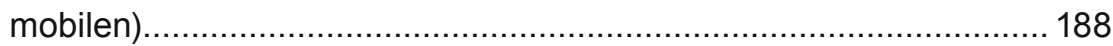

A5 Detaillierte Grundlagen zu Kapitel 5 (Lebenszyklusanalyse)............ 191

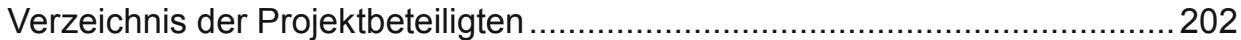

Verzeichnis der Begleitgruppenmitglieder.............................................. 203 


\section{Zusammenfassung}

Elektroautos gelten als Hoffnungsträger für eine nachhaltige oder zumindest weniger umweltbelastende Mobilität. Erste Stromfahrzeuge treten auf dem Markt bereits erfolgreich in Erscheinung. Generell erlauben es Elektrofahrzeuge, den energetischen Wirkungsgrad der Mobilität zu erhöhen und die Abhängigkeit von konventionellen Treibstoffen wie Benzin und Diesel zu senken. Gerade die Schweiz verfügt dank ihres Energiemixes, der sich zu einem grossen Teil aus der Wasserkraft speist, über günstige Voraussetzungen, um Elektrofahrzeuge umweltschonend anzutreiben. Ausserdem kann der geplante starke Ausbau erneuerbarer Stromproduktion durch Elektromobile als lokale Energiespeicher unterstützt werden.

\section{Weniger Treibhausgase durch effiziente Fahrzeuge}

Damit die ökologischen Stärken der alternativen Antriebsform zum Tragen kommen, müssen allerdings zahlreiche Bedingungen erfüllt sein. Der Massstab, an dem sich Elektroautos zu messen haben, ist umso strenger, als sich auch die konventionellen Fahrzeuge mit Verbrennungsmotoren technisch laufend weiter entwickeln, effizienter werden und weniger $\mathrm{CO}_{2}$ freisetzen.

Ein Kompaktwagen, der heute im Alltag noch durchschnittlich 7,5 Liter Benzin pro 100 Kilometer verbraucht, könnte im Jahr 2035 einen Verbrauch von nur noch 4,8 Litern erzielen, was einer Reduktion von mehr als einem Drittel entspricht. Der gleiche Kompaktwagen mit elektrischem Antrieb könnte seinen Strombedarf aufgrund von Verbesserungen bei Nebenverbrauchern wie Heizung oder Batterie-Konditionierung von heute $24 \mathrm{kWh}$ auf $16 \mathrm{kWh}$ pro $100 \mathrm{Kilo-}$ meter im Jahr 2035 drosseln und die $\mathrm{CO}_{2}$-Emissionen somit ebenfalls um gut 30 Prozent senken.

\section{Verlagerung der Umweltbelastung vom Betrieb der Fahrzeuge zu ihrer Herstellung und Entsorgung}

Dass die Ökobilanz von Elektroautos nicht wesentlich besser abschneidet als jene konventioneller Fahrzeuge, hängt damit zusammen, dass ein grosser Teil der Umweltbelastung bei der Herstellung der Vehikel anfällt: Berücksichtigt man ihren ganzen Lebenszyklus, entstehen bei den batteriebetriebenen Fahrzeugen 
bereits heute 90 Prozent der Treibhausgasemissionen bei der Produktion; bei einem Wagen der Mittelklasse mit Verbrennungsmotor sind es heute 25 Prozent, und auf längere Frist zeichnet sich eine Steigerung auf 40 Prozent ab.

In der Betriebsphase hängt die Umweltbelastung davon ab, wie viel Treibstoff die Fahrzeuge verbrauchen bzw. mit welchem Strommix Elektroautos angetrieben werden. Die Schweiz weist einen im Vergleich zu anderen Ländern $\mathrm{CO}_{2}$-armen Stromproduktionsmix auf, der auf Wasserkraft und Atomenergie beruht. Daher stösst ein mit Schweizer Elektrizität betriebenes Elektroauto heute 70 Prozent weniger Treibhausgase aus als ein vergleichbares Fahrzeug mit Verbrennungsmotor. Wenn Elektroautos hingegen den durchschnittlichen EU-Strommix tanken, der zu 52 Prozent auf fossilen Energieträgern beruht, vermindert sich die $\mathrm{CO}_{2}$-Reduktion gegenüber einem konventionellen Personenwagen auf nur 20 Prozent. Das umwelt- und energiepolitische Instrumentarium sollte daher zunehmend auf den gesamten Lebenszyklus von Fahrzeugen ausgedehnt werden. Nachhaltige Elektromobilität ist nur möglich, wenn die Ressourcen-Kreisläufe geschlossen werden.

\section{Bedeutung von Elektromobilität für die Schweiz}

Um die künftigen Auswirkungen der Elektromobilität für die Schweiz abzuschätzen, schlägt die Studie von TA-SWISS den Bogen vom einzelnen Auto zur gesamthaft durch die Schweizer Fahrzeugflotte verursachten Umweltbelastung. In drei Szenarien, die die Bandbreite möglicher Entwicklungspfade abstecken, wird die zukünftige Ausbreitung der Elektromobilität in der Schweiz modelliert. Die Studie erwartet im Vergleich zu Literaturwerten eine anfänglich eher langsame Verbreitung von Elektromobilität. So kann im Schnitt der Szenarien damit gerechnet werden, dass 2025 jeder zehnte Neuwagen elektrisch ist, 2035 wird bereits jeder zweite Neuwagen ein Elektroauto sein.

Basierend auf diesen Verbreitungsszenarien konnten die für die Jahre 2020, 2035 und 2050 erwarteten $\mathrm{CO}_{2}$-Emissionen abgeschätzt werden. Bei allen Szenarien resultiert bis im Jahr 2020 eine praktisch identische Reduktion der Treibhausgasemissionen aus dem Verkehr von 10 Prozent gegenüber heute und dies, obwohl mit einer Mobilitätszunahme von 24 Prozent gerechnet wird. Grössere Unterschiede zwischen den Elektromobilitäts-Szenarien ergeben sich ab 2035 - dann werden für das Business-as-Usual-Szenario 20 Prozent und für das optimistischste Szenario mehr als 30 Prozent Einsparungen an Treibhausgasen erwartet. 
Je stärker energiepolitische Massnahmen die Energieeffizienz der Neufahrzeuge fördern, desto eher nimmt die Elektromobilität zu. Denn Elektrofahrzeuge haben eine hohe Energieeffizienz, und insbesondere kleine bis mittlere Fahrzeuge lassen sich gut elektrifizieren - dank Fortschritten in der Batterietechnik mit künftig immer weniger Kompromissen. Eine gezielte, antriebsspezifische Förderung von Elektrofahrzeugen erscheint demnach nicht notwendig.

\section{Neue Finanzierungsmodelle gefragt}

Die Energieeffizienz der Strassenfahrzeuge wird künftig etwas stärker zunehmen als die Gesamtmobilität. Damit nehmen nicht nur die $\mathrm{CO}_{2}$-Emissionen ab, sondern auch die Erträge aus der Mineralölsteuer. Strom für Elektromobile lässt sich technisch kaum höher besteuern als «normaler» Strom. Je mehr Elektromobilität, desto grösser wird der Druck, auf eine fahrleistungsabhängige Besteuerung zu wechseln. Die Studie von TA-SWISS empfiehlt, solche fahrleistungsabhängige Steuermodelle auch von der Primärenergieeffizienz abhängig zu machen; dann läuft man nicht Gefahr, dass ein solcher Systemwechsel die Marktdurchdringung von Elektrofahrzeugen bremst. Um negativen Rückkopplungseffekten vorzubeugen, ist die Mobilität gesamthaft zu verteuern, sodass umweltfreundlichere und günstigere Fahrzeuge nicht zu einer Zunahme des Gesamtverkehrs führen.

\section{Bestens abgestütztes wissenschaftliches Fundament}

Die Studie von TA-SWISS beruht auf einer Vielzahl von Komponenten. Sie verwendet die gleichen Grundannahmen zu Bevölkerungswachstum und Mobilitätszunahme wie die Energieperspektiven des Bundesamts für Energie. Die Annahmen, welche den verschiedenen BFE-Szenarien zugrunde liegen, werden nach Möglichkeit übernommen. Diese Prognosen verbindet die Studie mit eigenen Kalkulationen zur technischen Entwicklung der unterschiedlichen Fahrzeugklassen und -komponenten und führt eine konsistente Bottom-Up-Berechnung durch. Die Studie malt damit gewissermassen die BFE-Szenarien aus und konkretisiert sie.

Die Berechnungen zur Marktpenetration der Elektrofahrzeuge berücksichtigen, dass für die nächsten Jahre die Produktionskapazitäten in der Automobilindustrie noch ein limitierender Faktor sein können. Ab zirka dem Jahr 2020 spielt nur noch eine Rolle, ob sich die angebotenen Elektrofahrzeuge gegenüber den ebenfalls jährlich besser werdenden Autos mit Verbrennungsmotor am Markt durchsetzen können. Dazu wird für jedes Jahr der schweizerische Neuwagen- 
markt simuliert, indem eine Million hypothetischer Haushalte aus konkreten konventionellen und elektrischen Modellvarianten einen Neuwagen kaufen müssen. Ausserdem analysiert die Studie den Lebenszyklus verschiedener Fahrzeugkategorien vom Kleinwagen bis zum Offroader und ermittelt die in den verschiedenen Phasen anfallende Umweltbelastung. Die hierzu verwendeten Modelle basieren auf den aktuellsten, wissenschaftlich abgestützten Umweltinventaren von Li-IonBatterien und einer im Rahmen des THELMA-Projekts entwickelten und für Elektromobilität optimierten Ökobilanzierungsmethode.

\section{Résumé}

Les voitures électriques suscitent l'espoir de voir émerger une mobilité durable ou, en tout cas, moins polluante. Les premiers véhicules électriques lancés sur le marché sont d'ores et déjà prometteurs. D'une manière générale, ce type de véhicule permet d'augmenter le rendement énergétique de la mobilité et de diminuer la dépendance aux carburants conventionnels tels que l'essence et le diesel. La Suisse dispose justement, grâce à son mix énergétique qui repose en grande partie sur l'énergie hydraulique, de conditions favorables pour promouvoir les véhicules électriques non polluants. En outre, la production de courant renouvelable, dont on prévoit une forte augmentation, peut s'appuyer sur des véhicules électriques comme accumulateurs d'énergie locaux.

\section{Moins de gaz à effet de serre grâce à des véhicules plus efficients}

L'exploitation des atouts écologiques de cette forme de propulsion alternative dépend toutefois de nombreuses conditions. Les critères sur lesquels repose l'évaluation des voitures électriques sont d'autant plus sévères que les véhicules conventionnels équipés de moteurs à combustion connaissent également un développement constant, qu'ils deviennent plus efficients et qu'ils dégagent moins de $\mathrm{CO}_{2}$.

Une voiture compacte qui consomme actuellement 7,5 litres d'essence sur 100 kilomètres en moyenne pourrait, en 2035, parcourir la même distance avec 4,8 litres seulement, ce qui représente une réduction de plus d'un tiers. La même voiture compacte dotée d'un moteur électrique pourrait quant à elle, grâce aux améliorations apportées aux équipements auxiliaires comme le chauffage ou le 
conditionnement des batteries, passer de $24 \mathrm{kWh}$ actuellement à $16 \mathrm{kWh}$ sur 100 kilomètres en 2035, ce qui représente également une diminution de 30 pour cent des émissions de $\mathrm{CO}_{2}$.

\section{Transfert de la charge environnementale de l'utilisation des véhicules au niveau de leur fabrication et élimination}

On constate que l'écobilan des voitures électriques n'est pas fondamentalement meilleur que celui des véhicules conventionnels. L'explication en est simple: une grande partie de la pollution est générée par la fabrication des véhicules ellemême: si l'on prend en effet en considération leur cycle de vie global, on constate que 90 pour cent des émissions de gaz à effet de serre sont générés actuellement lors de la production des véhicules électriques, alors que pour une voiture de moyenne catégorie équipée d'un moteur à combustion ce taux est aujourd'hui de 25 pour cent; on estime qu'à long terme ce même taux augmentera à 40 pour cent.

Durant la phase d'exploitation, la pollution dépend de la quantité de carburant consommée par les véhicules, respectivement du mix d'énergie avec lequel les voitures électriques fonctionnent. En comparaison internationale, la Suisse affiche un mix de production électrique pauvre en $\mathrm{CO}_{2}$ qui fait la part belle à l'énergie hydraulique et à l'énergie atomique. Une voiture électrique fonctionnant avec de l'électricité suisse émet aujourd'hui 70 pour cent moins de gaz à effet de serre qu'un véhicule comparable doté d'un moteur à combustion. $\mathrm{Si}$, en revanche, les voitures électriques font le plein avec le mix électrique moyen de l'UE, dont 52 pour cent proviennent de sources d'énergie fossiles, la réduction de $\mathrm{CO}_{2}$ n'est plus que de 20 pour cent par rapport aux voitures de tourisme conventionnelles. Les instruments de la politique environnementale et énergétique devraient donc être de plus en plus appliqués à l'ensemble du cycle de vie des véhicules. La mobilité électrique durable n'est en effet possible que dans le cadre d'un cercle de ressources vertueux.

\section{L’importance de la mobilité électrique pour la Suisse}

Pour pouvoir estimer les impacts futurs de la mobilité électrique pour notre pays, l'étude réalisée par TA-SWISS propose de passer de la pollution générée par chaque voiture individuelle à la pollution causée par l'ensemble du parc des véhicules suisse. La propagation de la mobilité électrique en Suisse est modélisée au moyen de trois scénarios qui couvrent l'ensemble des voies de développe- 
ment possibles. Selon cette étude et les scénarios qu'elle avance, la propagation de la mobilité électrique devrait être plutôt lente au début. Si, à l'horizon 2025, une voiture neuve sur dix sera électrique, une sur deux le sera en 2035.

Ces scénarios permettent ensuite d'estimer les émissions de $\mathrm{CO}_{2}$ pour les années 2020, 2035 et 2050. Tous les scénarios envisagés prévoient, d'ici 2020, une réduction pratiquement identique des émissions de gaz à effet de serre produites par le trafic, soit de 10 pour cent par rapport à aujourd'hui, et ce bien que l'augmentation de la mobilité soit estimée à 24 pour cent. Les différences les plus importantes entre les scénarios se dessineront à partir de 2025, car le scénario «business as usual» prévoit des économies de gaz à effet de serre de 20 pour cent et le scénario le plus optimiste plus de 30 pour cent.

Plus les mesures de politique énergétique promeuvent l'efficience énergétique des véhicules neufs et plus la mobilité électrique a tendance à augmenter. En effet, les véhicules électriques affichent une efficience énergétique élevée, et notamment les véhicules petits à moyens peuvent être facilement électrifiés, grâce aux progrès accomplis dans le domaine de la technique des batteries, technique qui fera de moins en moins de compromis à l'avenir. Un encouragement ciblé, orienté spécifiquement vers les moteurs des véhicules électriques ne semble dès lors pas être nécessaire.

\section{On recherche de nouveaux modèles de financement}

L'efficience énergétique des véhicules augmentera à l'avenir davantage que la mobilité globale. En d'autres termes, non seulement les émissions de $\mathrm{CO}_{2}$ diminueront, mais également les recettes provenant des impôts sur les huiles minérales. D'un point de vue technique, l'électricité destinée aux véhicules électriques ne peut pratiquement pas être imposée à un taux plus élevé que l'électricité «normale». Plus la mobilité électrique se généralisera, plus la pression sera forte pour passer à une imposition en fonction de la performance des véhicules. L'étude de TA-SWISS recommande de faire dépendre de tels modèles d'imposition également de l'efficience au niveau de l'énergie primaire; on éviterait ainsi le risque qu'un tel changement de système ne freine la pénétration du marché par les véhicules électriques. Afin de prévenir des effets de rétroaction non souhaités, il vaut imposer globalement la mobilité, de sorte que les véhicules moins polluants et plus avantageux n'entraînent pas une augmentation du trafic global. 


\section{De solides bases scientifiques}

L'étude de TA-SWISS repose sur un grand nombre d'éléments. Elle utilise les mêmes hypothèses de base concernant la croissance de la population et l'augmentation de la mobilité, ainsi que les perspectives énergétiques de l'Office fédéral de l'énergie. Les hypothèses sur lesquelles reposent les différents scénarios de l'OFEN sont reprises dans la mesure du possible. L'étude combine ces pronostics avec des calculs en propre concernant le développement des différentes classes de véhicules et des composants, et propose un calcul bottom-up cohérent. Elle étoffe en quelque sorte les scénarios de l'OFEN et les concrétise.

Les calculs de la pénétration du marché des véhicules électriques tiennent compte du fait que les capacités de production de l'industrie automobile pourront être un facteur limitant ces prochaines années. A partir de l'année 2020 environ, la seule question importante sera celle de savoir si les véhicules électriques proposés pourront s'imposer sur le marché au détriment des voitures équipées de moteur à combustion, qui s'améliorent elles aussi chaque année. A cet effet, le marché suisse des voitures neuves fait l'objet d'une simulation annuelle, sur la base de l'hypothèse selon laquelle un million de ménages achète une nouvelle voiture parmi les variantes de modèles conventionnels et électriques.

L'étude analyse en outre le cycle de vie de différentes catégories de véhicules, de la petite voiture au tout-terrain, et estime la pollution générée au cours des différentes phases. Les modèles utilisés dans ce contexte reposent sur des inventaires environnementaux les plus récents, scientifiquement fondés, des batteries lithium-ion et sur une méthode d'écobilan développée dans le cadre du projet THELMA et optimisée pour la mobilité électrique.

\section{Riassunto}

Le automobili elettriche fanno ben sperare in una mobilità sostenibile o se non altro meno inquinante. I primi veicoli elettrici vengono già immessi con successo sul mercato. In generale i veicoli elettrici consentono di aumentare il grado di efficacia energetica della mobilità e di ridurre la dipendenza dai carburanti tradizionali quali la benzina e il diesel. Proprio la Svizzera presenta dei presupposti favorevoli per promuovere i veicoli elettrici a basso impatto ambientale, grazie al 
suo mix energetico, composto in parte di energia idroelettrica. Inoltre il previsto forte incremento nella produzione di energie rinnovabili può essere agevolato dall'utilizzo di veicoli elettrici come accumulatori locali di energia.

\section{Meno gas serra grazie a veicoli più efficienti}

Affinché si concretizzino i vantaggi ecologici di questa forza motrice alternativa, devono tuttavia essere soddisfatte numerose condizioni. I criteri per la valutazione delle automobili elettriche diventano tanto più rigorosi quanto maggiori sono la continua evoluzione tecnica dei veicoli tradizionali con motori a combustione, la relativa efficienza e la riduzione di emissioni di $\mathrm{CO}_{2}$.

Un'automobile compatta, che attualmente consuma in media 7,5 litri di benzina ogni 100 chilometri, potrebbe raggiungere nel 2035 un consumo di soli 4,8 litri, pari a una riduzione di più di un terzo. La stessa automobile compatta fornita di motore elettrico potrebbe, in seguito al miglioramento di dotazioni ausiliarie quali ad esempio il riscaldamento o la calibrazione della batteria, ridurre il proprio fabbisogno elettrico dagli attuali $24 \mathrm{kWh}$ a $16 \mathrm{kWh}$ ogni 100 chilometri nel 2035, contenendo pertanto anche le emissioni di $\mathrm{CO}_{2}$ di un buon 30 percento.

\section{Trasferimento dell'inquinamento ambientale dalla fase di utilizzo dei vei- coli alla loro produzione e smaltimento}

II life cycle analysis delle automobili elettriche non risulta fondamentalmente migliore rispetto ai veicoli tradizionali; questo dipende dal fatto che gran parte dell'inquinamento ambientale è da ricondurre alla fase di produzione dei veicoli. Se si considera l'intero ciclo di vita, si constata che attualmente nei veicoli a batteria il 90 percento delle emissioni di gas serra è generato durante la produzione; per un'automobile di classe media con motore a combustione questa percentuale è oggi al 25 percento, mentre a lungo termine si prospetta un incremento al 40 percento.

Nella fase d'utilizzo l'inquinamento ambientale dipende da quanto carburante consumano i veicoli ovvero dal mix di elettricità con cui sono alimentate le vetture. Rispetto ad altri paesi, la Svizzera presenta un mix di energia di produzione a basso contenuto di $\mathrm{CO}_{2}$, basato sull'energia idroelettrica e sull'energia nucleare. Perciò un'automobile elettrica azionata con elettricità svizzera genera oggi il 70 percento di gas serra in meno rispetto a un veicolo equivalente a combustione. Se invece le automobili elettriche vengono alimentate con il mix di energia elettrica 
medio dell'UE, che consta per il 52 percento di fonti energetiche fossili, la riduzione di $\mathrm{CO}_{2}$ rispetto a un'automobile tradizionale è pari al solo 20 percento. L'insieme di strumenti di politica ambientale ed energetica dovrebbe quindi essere sempre più applicato all'intero ciclo di vita dei veicoli. La mobilità elettrica sostenibile è possibile solo se si considerano i cicli delle risorse nella loro interezza.

\section{Importanza della mobilità elettrica per la Svizzera}

Con lo scopo di valutare i futuri effetti della mobilità elettrica per la Svizzera, lo studio di TA-SWISS considera l'inquinamento ambientale dalla singola automobile fino all'intera flotta di veicoli svizzeri. Lo studio prevede la futura diffusione della mobilità elettrica in Svizzera mediante tre scenari che tracciano diversi possibili modelli si sviluppo. Diversamente dai valori indicati nella letteratura, lo studio prevede inizialmente una diffusione piuttosto lenta della mobilità elettrica. Facendo una media tra i diversi scenari, si può ipotizzare che nel 2025 un'automobile nuova su dieci sarà elettrica, mentre nel 2035 il rapporto salirà già a una su due.

Sulla base di questi scenari è stata fatta una stima delle emissioni di $\mathrm{CO}_{2}$ per il 2020,2035 e il 2050. In tutti e tre gli scenari risulta che fino al 2020 ci sarà una riduzione pressoché identica, pari al 10 percento rispetto a oggi, delle emissioni di gas serra prodotti dal traffico, e questo nonostante si preveda un incremento del 24 percento della mobilità. Maggiori differenze tra gli scenari si ottengono a partire dal 2035 - quando per lo scenario Business-as-Usual sono attese flessioni dei gas serra del 20 percento, mentre per lo scenario più ottimista di oltre il 30 percento.

Più incisive sono le misure di politica energetica che richiedono efficienza energetica dei nuovi veicoli, maggiore è la diffusione della mobilità elettrica. I veicoli elettrici presentano infatti un'elevata efficienza energetica; soprattutto i veicoli di piccola e media grandezza possono essere ben elettrificati grazie ai progressi compiuti nella tecnologia delle batterie, tecnologia che in futuro sarà caratterizzata da sempre meno compromessi. Non appare pertanto necessaria una promozione mirata e specifica dei motori dei veicoli elettrici.

\section{L’opportunità di attivare nuovi modelli di finanziamento}

L'efficienza energetica dei veicoli stradali aumenterà in futuro in maniera leggermente superiore rispetto alla mobilità generale. Diminuiranno così non solo le 
emissioni di $\mathrm{CO}_{2}$, ma anche i proventi dalle tasse sui carburanti. Da un punto di vista tecnico l'elettricità per le automobili elettriche non può essere tassabile in misura maggiore dell'elettricità «normale». Maggiore sarà la mobilità elettrica, maggiore sarà la pressione a passare a una tassazione basata sulla performance dei veicoli. Lo studio di TA-SWISS raccomanda quindi di subordinare questo genere di modelli fiscali basati sulla quantità di chilometri percorsi anche all'efficienza a livello dell'energia primaria; non si corre così il rischio che il nuovo sistema di tassazione freni la penetrazione dei veicoli elettrici sul mercato. Per evitare effetti boomerang negativi occorre rincarare la mobilità nel suo complesso, in maniera tale che veicoli più ecologici ed economici non generino un incremento del traffico nel suo complesso.

\section{Una solida base scientifica}

Lo studio di TA-SWISS poggia su una molteplicità di elementi. Utilizza le medesime ipotesi di fondo relative alla crescita della popolazione e all'aumento della mobilità nonché le prospettive energetiche dell'Ufficio federale dell'energia (UFE). Le ipotesi alla base dei diversi scenari dell'UFE vengono riprese ogni qualvolta possibile. Lo studio combina queste previsioni coni propri calcoli relativi all'evoluzione tecnica delle diverse classi di veicoli e dei componenti ed effettua un calcolo sistematico bottom-up. Lo studio raffigura quindi in un certo qual modo gli scenari dell'UFE, concretizzandoli.

I calcoli della penetrazione sul mercato dei veicoli elettrici tengono conto che per i prossimi anni le capacità di produzione dell'industria automobilistica possano essere ancora un fattore limitante. A partire dal 2020 circa diviene invece determinante sapere se i veicoli elettrici offerti si possono affermare sul mercato rispetto alle automobili con motore a combustione, in miglioramento di anno in anno. A tale fine l'evoluzione del mercato svizzero di automobili nuove viene simulata, ipotizzando che ogni anno un milione di economie domestiche debbano comprare un'automobile nuova tra varianti di modelli tradizionali ed elettrici.

Lo studio analizza infine il ciclo di vita di diverse categorie di veicoli, dalle automobili di piccole dimensioni fino ai fuoristrada, e stima l'inquinamento ambientale generato nelle diverse fasi. I modelli utilizzati a tal fine si basano sugli inventari ambientali più aggiornati e scientificamente fondati relativi alle batterie Li-lon e su un metodo di analisi del ciclo di vita sviluppato nel quadro del progetto THELMA, ottimizzato per la mobilità elettrica. 


\section{Summary}

Electric cars are seen as the great hope for a sustainable or at least less polluting mode of transport. The first electric cars to appear on the market are already a success. Generally speaking, electric cars increase the energy efficiency of travel and reduce dependency on conventional fuels such as petrol and diesel. Thanks to its energy mix, of which a large part comes from hydroelectric power, Switzerland has the right conditions to generate environmentally friendly energy for electric vehicles. Furthermore, the planned major expansion of renewable energy production may be supported by electric vehicles as a form of local energy storage.

\section{Lower greenhouse gas emissions with efficient vehicles}

However, in order to reap the ecological benefits of alternative fuels, many conditions need to be met. The benchmark against which electric cars are measured is becoming even more rigorous as conventional combustion engine vehicles are continually technically refined to make them more efficient and ensure lower $\mathrm{CO}_{2}$ emissions.

By 2035, a compact car which today uses on average 7.5 litres of petrol per 100 kilometres could be using only 4.8 litres; this corresponds to a reduction of more than one third. The same compact car which runs on electricity could cut its energy use from $24 \mathrm{kWh}$ to $16 \mathrm{kWh}$ per 100 kilometres by 2035 through improvements in auxiliary systems such as heating and battery conditioning. This corresponds to a reduction of around 30 percent in $\mathrm{CO}_{2}$ emissions.

\section{Shift in environmental pollution from operation of vehicles to their manu- facture and disposal}

One major reason why the life cycle assessment of electric cars is not substantially better than that of conventional vehicles is due to environmental pollution during the manufacture of the car: if we take into consideration the entire life cycle, 90 percent of greenhouse gas emissions from battery-powered vehicles are produced during manufacture. This compares with 25 percent for mid-sized cars with combustion engines today, increasing to 40 percent over the longer term. 
During operation, environmental pollution depends on how much fuel the vehicle consumes, or in the case of electric cars, the electricity mix. In comparison with other countries, Switzerland has one of the lowest $\mathrm{CO}_{2}$ producing electricity mixes, based on hydroelectric and nuclear power. Operating an electric car powered by Swiss electricity therefore produces 70 percent fewer greenhouse gas emissions than a comparable combustion engine vehicle. By contrast, if the electric car is charged using the average EU electricity mix, of which 52 percent comes from fossil fuels, the $\mathrm{CO}_{2}$ reduction in comparison with a conventional car is reduced to 20 percent. Consequently, environmental and energy policy instruments should be increasingly extended to cover the entire vehicle life cycle. Sustainable electromobility is only possible if the resource life cycle is closed.

\section{What electromobility means for Switzerland}

In order to assess the future impact of electromobility in Switzerland, the TASWISS study links individual cars with the environmental pollution caused by the entire Swiss vehicle fleet. Three scenarios based on the range of possible development paths are applied to model the future expansion of electromobility in Switzerland. Compared with literature values, the study initially expects a rather slow uptake of electromobility. Based on these scenarios, we can calculate that on average, in 2025 one in ten new cars will run on electricity, and in 2035 every second new car will be an electric car.

On the basis of this distribution scenario, we can estimate the expected $\mathrm{CO}_{2}$ emissions in 2020, 2035 and 2050. In all the scenarios there is an almost identical 10 percent reduction in greenhouse gas emissions from transport by 2020 compared with today, despite a calculated 24 percent increase in mobility. From 2035 , there are major variations between the electromobility scenarios: the business-as-usual scenario predicts a 20 percent reduction in greenhouse gases, while the optimistic scenario predicts a 30 percent reduction.

The more actively energy policy measures promote energy efficiency for new cars, the more likely it is that electromobility will increase. Electric cars have high energy efficiency and small and mid-sized cars in particular are well suited to running on electricity - thanks to advances in battery technology, which will mean increasingly fewer compromises in future. Accordingly, targeted drive-specific support for electric cars does not seem to be necessary. 


\section{New financing model required}

In future, the energy efficiency of road vehicles will improve more rapidly than the rate of total mobility. This will mean not only a reduction in $\mathrm{CO}_{2}$ emissions, but also in income from fuel taxes. Electricity for electric cars is not taxed any higher than «normal» electricity. The higher the rate of electromobility, the greater the pressure to switch to a taxation system based on kilometres travelled will become. The TA-SWISS study recommends also basing this kind of distance travelled-based taxation models on primary energy efficiency; this would avoid the risk of such a system change slowing the market penetration of electric cars. In order to prevent negative feedback effects, an increase in the general cost of mobility is needed to ensure that more environmentally friendly and less expensive vehicles do not result in an increase in the general traffic.

\section{Extremely well supported scientific basis}

The TA-SWISS study is based on a range of components. It applies the same underlying assumptions on population growth and increase in mobility as the Energy Perspectives of the Swiss Federal Office of Energy (SFOE). The assumptions on which the various SFOE scenarios are based are adopted as far as possible. The study links this projection with its own calculations on the technical development of various vehicle classes and components performing a consistent bottom-up calculation. Thus it describes and more specifically substantiates the scenarios outlined by the SFOE.

The calculations for market penetration by electric cars take into consideration the potentially limiting factor of production capacity in the automotive industry in the years ahead. From approximately 2020 , it will remain to be seen whether the electric cars available are able to prevail over combustion engine cars which are also constantly improving year-on-year. To this end, the Swiss new car market is simulated for each year, in which a million hypothetical households must purchase a new car, choosing between existing conventional and electric car models.

The study also analyses the life cycle of various vehicle categories from small cars to offroaders, and determines the environmental pollution resulting from each phase. The models applied here were based on the latest, scientifically supported life cycle inventories of Li-ion batteries, and on life cycle assessment methods developed within the framework of the THELMA project and optimised for electrical mobility. 


\section{Executive Summary}

Elektrofahrzeuge können einen bedeutenden Beitrag zu einem umweltfreundlicheren Verkehrssystem leisten, auch wenn die Vorteile von Elektromobilen gegenüber modernen Fahrzeugen mit Verbrennungsmotoren weniger gross sind als oft angenommen. Dies zeigt eine Untersuchung der erwarteten Entwicklung in den nächsten Jahrzehnten anhand einer Modellierung des Konsumentenverhaltens, verschiedener Szenarien sowie von Lebenszyklusanalysen. Entscheidend für eine positive Umweltbilanz der Elektrofahrzeuge ist vor allem eine umweltfreundliche Stromproduktion. Aber auch, wenn in den nächsten 40 Jahren vorübergehend vermehrt Strom mit Gaskraftwerken erzeugt werden muss, wie es die Energiestrategie des Bundes vorsieht, bleibt eine rasche Verbreitung von Elektrofahrzeugen aus Klimasicht attraktiv. Da auch Fahrzeuge mit Verbrennungsmotor in den nächsten Jahrzehnten deutlich effizienter werden sollten, dürfte der $\mathrm{CO}_{2}$-Ausstoss der schweizerischen Autoflotte trotz gesteigerter $\mathrm{Ge}$ samtmobilität zurückgehen. Szenarienrechnungen zeigen, dass bei einer Politik der Förderung der Effizienz im Strassenverkehr allgemein der Anteil der Elektromobilität automatisch zunimmt, was zu einem weiteren Rückgang des Energieverbrauchs führt. Ein weiterer Vorteil der Elektromobilität liegt darin, dass sich die Abhängigkeit von Erdöl reduziert und die Integration lokal produzierter Elektrizität unterstützt wird.

Neue Geschäftsmodelle (neue Car-Sharing-Ansätze, vermehrte Pay-as-youdrive-Modelle) und neue Anbieter (wie Energieversorgungsunternehmen und konzessionierte Transportunternehmen) können den Trend hin zu mehr Elektromobilität zusätzlich erleichtern. Sie können auch sicherstellen, dass vermehrte Elektromobilität den öffentlichen Verkehr (ÖV) stärkt und nicht konkurrenziert, namentlich auf Langstrecken und in Ballungszentren.

Da wir davon ausgehen, dass die Energieeffizienz der künftigen Fahrzeuge mit konventionellem Verbrennungsmotor so stark verbessert wird, dass der Energiebedarf der steigenden Gesamtmobilität überkompensiert wird, dürften sowohl der $\mathrm{CO}_{2}$-Gesamtausstoss als auch die Mineralölsteuereinnahmen zurückgehen. Dies kann zu Finanzierungslücken führen. Hohe Priorität hat daher ein rascher Übergang von der Mineralölbesteuerung zu einem Road- oder Mobility-Pricing, unabhängig davon, wie schnell und wie weit sich Elektromobilität verbreiten wird. Bereits eine erhöhte Energieeffizienz konventioneller Antriebskonzepte geht mit 
einer Verlagerung der Umweltbelastung vom Betrieb in Richtung Herstellung und Lebensende einher, und neben der Energie geraten auch die benötigten nicht energetischen Ressourcen vermehrt in den Fokus. Die Elektromobilität verstärkt diese Trends nochmals deutlich, namentlich wegen der benötigten Elektronik und der Batteriesysteme. Nachhaltige Elektromobilität erfordert daher verstärkt, dass das umwelt- und energiepolitische Instrumentarium auf den gesamten Lebenszyklus ausgedehnt wird und namentlich, dass dadurch ein Schliessen der Kreisläufe der verwendeten Metalle gewährleistet ist.

Elektromobilität gilt vielerorts als die grüne Verkehrstechnik par excellence, die den Weg in eine moderne nachhaltige Welt mit erneuerbaren Energien ebnet. TA-SWISS, das Zentrum für Technologiefolgen-Abschätzung der Akademien der Wissenschaften Schweiz, hat daher 2011 eine Studie zu den «Chancen und Risiken der Elektromobilität» in Auftrag gegeben. Sie soll der Frage nachgehen, inwiefern die in die Elektromobilität gesetzten Hoffnungen gerechtfertigt sind. Im Hinblick auf die Nachhaltigkeit und unerwünschte Nebenwirkungen soll die Studie insbesondere den "ganzen Lebenszyklus» von Elektrofahrzeugen und Infrastruktur, von der Produktion über die Nutzung bis zur Entsorgung berücksichtigen, sie soll wirtschaftliche Fragen in Betracht ziehen und schliesslich Handlungsempfehlungen vorlegen.

\section{Das Auto der Zukunft}

Ob und wie der breite Markteintritt der Elektromobilität gelingen wird, hängt stark von der technischen und ökonomischen Weiterentwicklung einzelner Schlüsselkomponenten ab. Obwohl die technischen Grundlagen weitgehend bekannt sind, ist unklar, wie diese Faktoren in Zukunft zusammenspielen und damit den Erfolg oder Misserfolg der Elektromobilität beeinflussen werden. Ein Ziel dieser Studie war deshalb, das gesamthafte zukünftige Verbesserungspotenzial von elektrischen und konventionellen Fahrzeugen nach einheitlicher Methodik abzuschätzen.

\section{Prognose der technologischen Entwicklung}

Die Batterie ist die teuerste Komponente eines Elektrofahrzeugs und damit - zusammen mit der beschränkten Reichweite - wohl der wichtigste limitierende Faktor für die Penetration von Elektromobilität. Die Entwicklung von Kosten und 
Leistung der Batterien bestimmen daher die zukünftige Verbreitung von Elektrofahrzeugen wesentlich mit. Bis 2035 kann mit einer Zunahme der Batteriekapazität von 100 auf $300 \mathrm{Wh} / \mathrm{kg}$ bei einer gleichzeitigen Reduktion der Kosten von 900 auf 250 US $\$ / k W h$ gerechnet werden. Bei gleichbleibendem Batteriegewicht (300 Kilogramm) wird dies zu einer wesentlichen Erhöhung der elektrischen Reichweite von heute rund 150 auf gegen 600 Kilometer für batteriebetriebene Elektrofahrzeuge (BEV) führen.

Im Gegensatz zu den Batterien sind Elektromotoren und Leistungselektronik bereits heute sehr energieeffizient. Obwohl sich auch diese Technologien noch weiter verbessern lassen, sind hier in den nächsten Jahrzehnten keine grösseren Effizienzgewinne mehr zu erwarten. Anders sieht es dagegen bei den $\mathrm{Ne}-$ benverbrauchern im Elektrofahrzeug aus. Da der Elektromotor nur wenig Abwäme produziert, muss der ganze Heizenergiebedarf über die Batterie gedeckt werden, was die Reichweite von BEV massiv reduzieren kann. Ebenfalls zusätzlichen Strom kostet die Klimatisierung der Batterie, welche oftmals nur in einem engen Temperaturbereich betrieben werden darf, und schliesslich können ineffiziente Ladegeräte bis zu 20 Prozent des Strombedarfs verschwenden.

Werden die verschiedenen Verbesserungsmassnahmen realistisch kombiniert, kann der zukünftige Effizienzgewinn modelliert werden. Der gleiche Kompaktwagen mit elektrischem Antrieb könnte somit seinen Strombedarf von heute $24 \mathrm{Ki}$ lowattstunden auf 16 Kilowattstunden pro 100 Kilometer im Jahr 2035 reduzieren, was einer Einsparung von 30 Prozent entspricht. Überraschend ist, dass das prozentuale Verbesserungspotenzial für Elektrofahrzeuge eher kleiner ist als für Fahrzeuge mit Verbrennungsmotor (ICE). Ein Kompaktwagen, der heute im Alltag 7,5 Liter Benzin pro 100 Kilometer verbraucht, könnte im Jahr 2035 auf einen Verbrauch von 4,8 Liter kommen, was einer Reduktion von mehr als einem Drittel entspricht.

\section{Trend zu geringerer Umweltbelastung}

Basierend auf der erwarteten technologischen Entwicklung wurde die Ökobilanz der zukünftigen Fahrzeuge berechnet. Abbildung 1 zeigt, dass die heutige Treibhausgasbilanz von ICE-Mobilität um Faktoren höher liegt, als von Elektromobilität, die mit Strom aus Schweizer Produktion und Import von Kernenergie gemäss der Energieperspektive 2050 des Bundes (BFE-Szenario) betrieben wird. Das zukünftige Reduktionspotenzial ist jedoch bei ICE-Fahrzeugen höher als bei $\mathrm{BEV}$, welche aufgrund von grösserem Produktionsaufwand und $\mathrm{CO}_{2}$-reicherem 
Strom in Zukunft etwas mehr Treibhausgasemissionen ausstossen werden. Die Emissionen durch die Fahrzeugproduktion werden leicht steigen, da für den angestrebten Leichtbau vermehrt Aluminium und Kompositmaterialien eingesetzt werden.

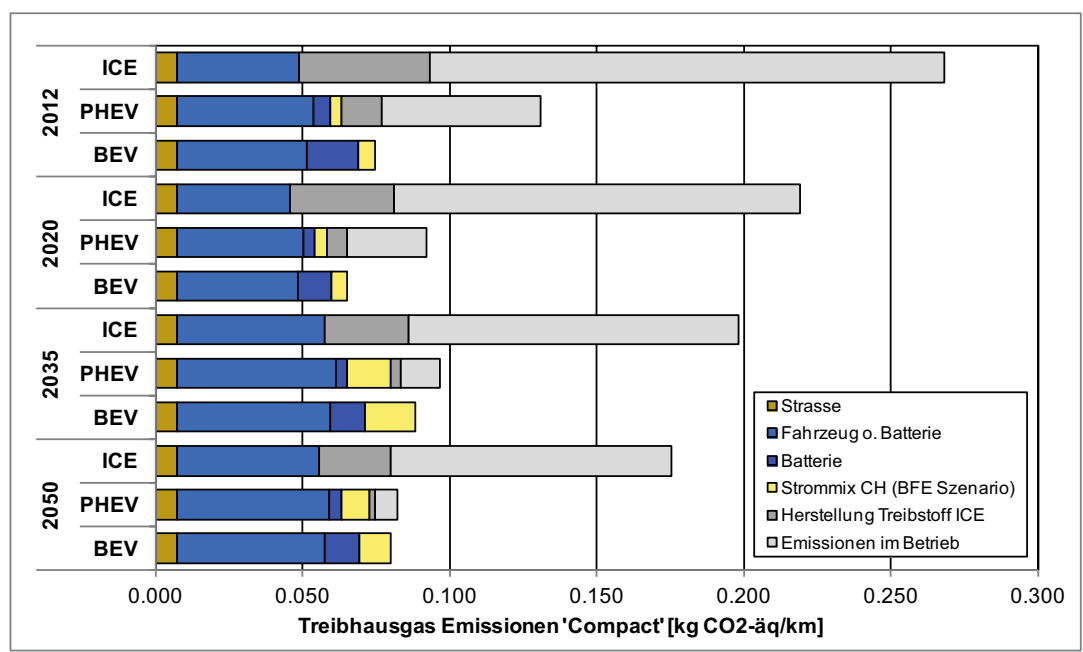

Abbildung 1: $\quad$ Lebenszyklus-Treibhausgasemissionen für die drei untersuchten Antriebsstränge (ICE, PHEV, BEV) und die vier Zeitpunkte (2012/2020/2035/2050) für die Fahrzeugklasse «Kompakt».

Die Abbildung 1 zeigt auch, dass bei BEV und Plug-in-Hybriden (PHEV) der weitaus grösste Teil der Umweltbelastung bei der Herstellung von Fahrzeug und Batterie anfällt. Die schrumpfende Kluft zwischen fossil getriebenen Fahrzeugen und reinen Elektromobilen zeigt sich auch beim Ressourcenverbrauch in Abbildung 2. Beim Kompaktwagen Golf zum Beispiel halbiert sich der Unterschied bis im Jahr 2050 faktisch von einem heutigen Faktor 3 auf 1,5.

Eine ganz entscheidende Rolle für die Nachhaltigkeit spielt sodann, wie die Elektrizität, mit der die Fahrzeuge angetrieben werden, hergestellt wird. Legt man den Rechnungen den Schweizer Strommix zugrunde, wie er heute aus Wasserkraft und Kernenergie sowie in deutlich kleinerem Masse aus fossilen Quellen und erneuerbaren Energieanlagen erzeugt wird, erscheint die Elektromobilität aus Sicht der Umwelt- und Klimabelastung deutlich attraktiver, als wenn der Strommix eingesetzt wird, welcher derzeit in Europa im Durchschnitt zur Ver- 
wendung kommt. Der Anteil erneuerbarer Energiequellen wie der Wasserkraft, die in der Schweiz knapp 40 Prozent der Elektrizität liefert, ist im europäischen Strom deutlich geringer, dafür ist der Anteil aus fossilen Quellen mit gut 50 Prozent, nicht zuletzt aus Kohle, erheblich grösser. Ähnlich hoch ist derzeit auch auf globaler Ebene der fossile Anteil am Strom. Werden Elektrofahrzeuge mit derartigem Strom betrieben, sind sie, wie Sensitivitätsanalysen zeigen, de facto nicht umwelt- und klimafreundlicher als entsprechende moderne Vehikel mit optimierten Verbrennungsmotoren. So schrumpft die Reduktion der Klimagasemissionen gegenüber einem fossil getriebenen Auto in der Kompaktklasse von 70 auf 20 Prozent, wenn statt dem heutigen Schweizer Produktionsmix der europäische Strom getankt wird (siehe Kapitel 5.6.2).

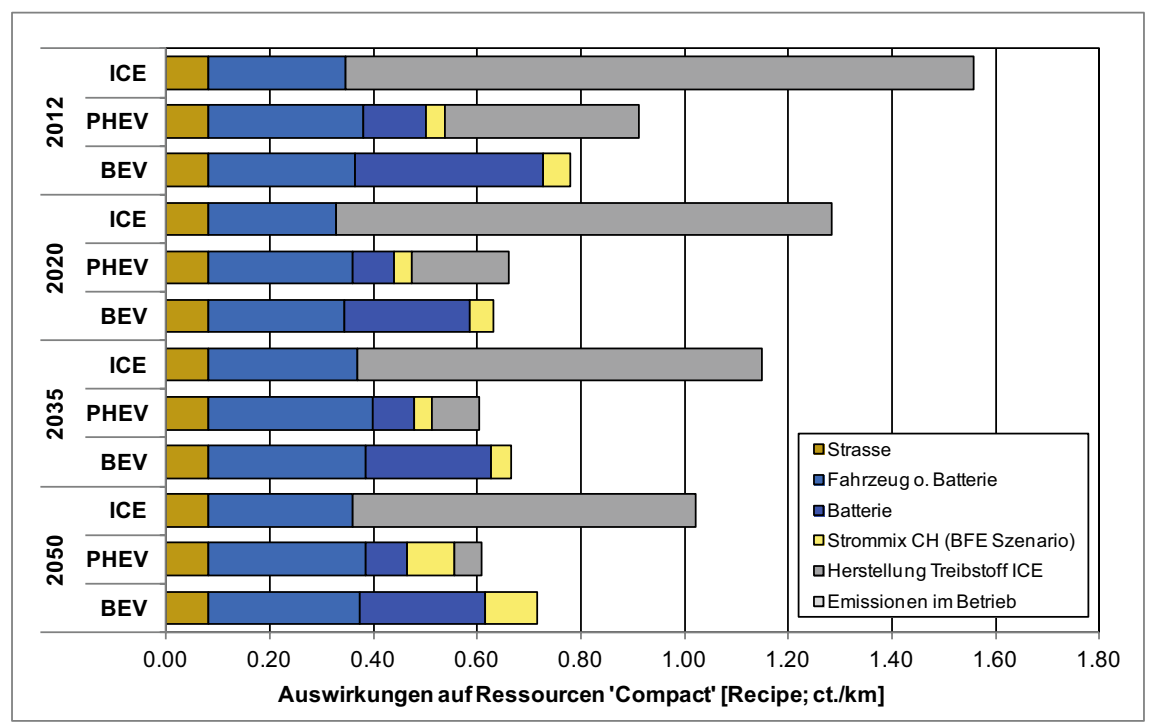

Abbildung 2: $\quad$ Bewertung des Ressourcenverbrauchs nach der ReCiPe-Methodik für die drei untersuchten Antriebsstränge (ICE, PHEV, BEV) und die vier Zeitpunkte (2012/2020/2035/2050) für die Fahrzeugklasse «Kompakt». 

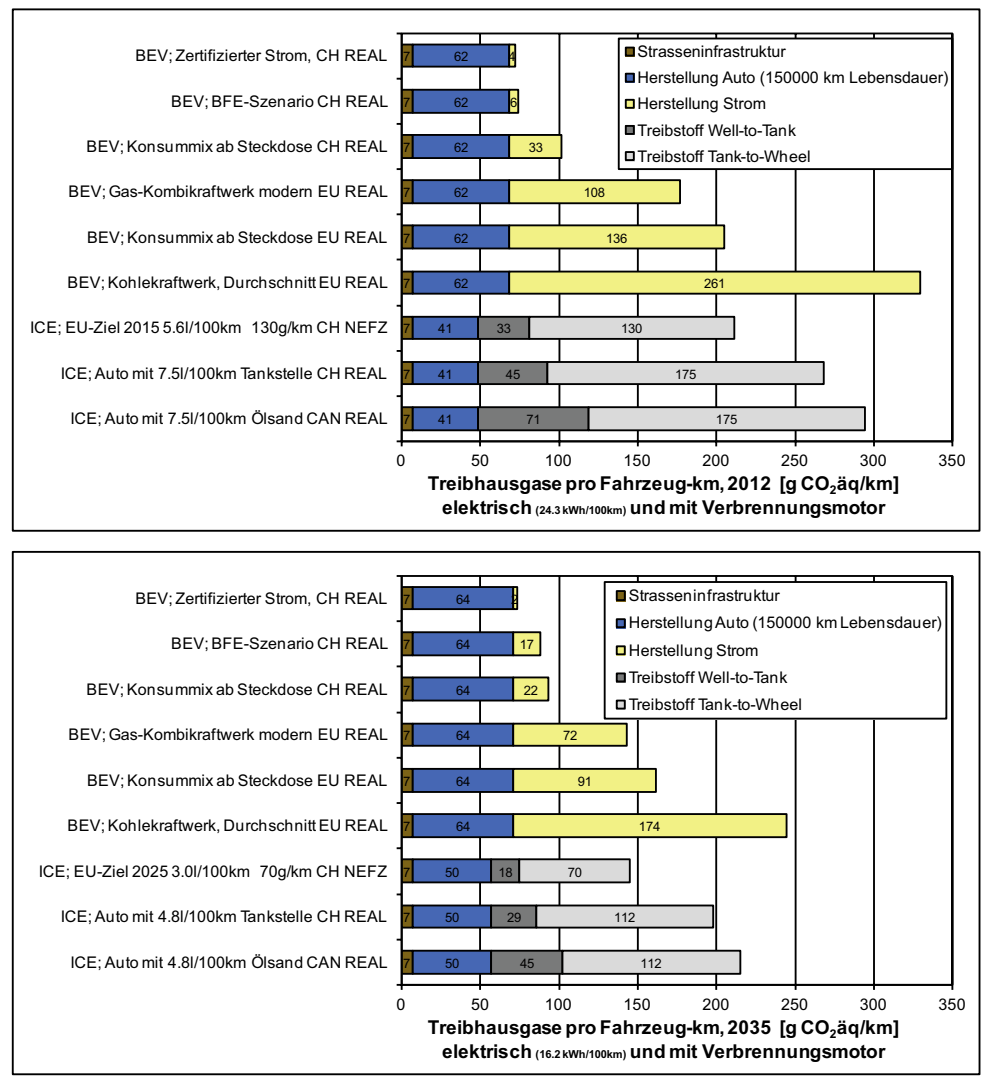

Abbildung 3: $\quad$ Lebenszyklus-Treibhausgasemissionen für zwei untersuchte Antriebsstränge (ICE, BEV) und zwei Zeitpunkte (2012/2035). Für das Elektrofahrzeug im Alltagsbetrieb (REAL) wurde der Strommix variiert von zertifiziertem Ökostrom bis Kohlestrom. Beim ICE Fahrzeug im Alltagsbetrieb (REAL) wurde die Benzinqualität variiert (Tankstellenmix $\mathrm{CH}$ bzw. Benzin aus kanadischen Ölsanden). Zum Vergleich wird ein ICE Fahrzeug gemäss den Zielvorgaben der EU im NEFZ-Fahrzyklus gezeigt. 


\section{Elektromobilität in der Schweiz}

Um die Bedeutung der Elektromobilität für die Schweiz aufzuzeigen, wurden mittels Modellrechnungen für drei Szenarien die Anteile der Fahrzeugtypen (fossil und elektrisch betrieben) für die Jahre bis Mitte des Jahrhunderts quantitativ abgeschätzt. Als Vorgabe dienten die Energieverbrauchsziele der EU und der Schweiz für die Personenwagenneuzulassungen (Mittelwert für 2015: $130 \mathrm{~g} \mathrm{CO}_{2} / \mathrm{km}$; ab 2020: $95 \mathrm{~g} \mathrm{CO}_{2} / \mathrm{km}$ ), welche die Effizienzentwicklung der Fahrzeuge mit Verbrennungsmotor steuern (Kapitel 4.2.4). Die kurzfristig bis 2020 überhaupt mögliche Produktion von Elektrofahrzeugen wurde abgeschätzt, stellt aber für keines der drei Szenarien einen limitierenden Faktor dar - das Hauptwachstum setzt in allen drei Szenarien erst später ein.

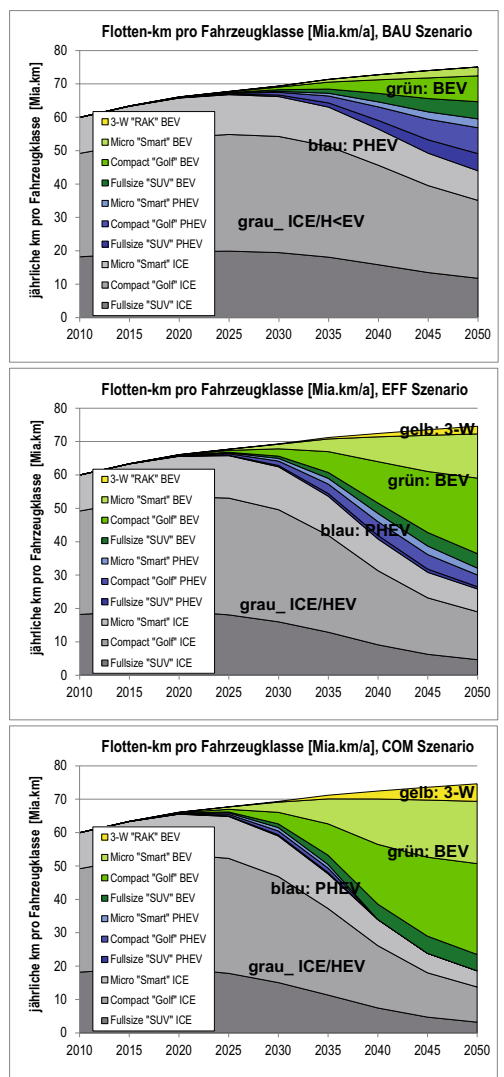

Abbildung 4: Flottenkilometer der verschiedenen Fahrzeugklassen und Antriebstypen. 
Für die drei Szenarien BAU, EFF und COM wurde angenommen, dass sich die Elektromobile unterschiedlich schnell weiterentwickeln (siehe Kapitel 2.6). In EFF und COM steigen zudem die Energiepreise stark an und die Batterien werden schneller besser und günstiger. Die Preise der Elektromobile fallen, sie werden zunehmend konkurrenzfähig mit den konventionellen Verbrennerfahrzeugen (welche sich aber ebenfalls stetig weiter entwickeln). Auch wird Sicherheit ein aktives Feld der Politik - Car-to-Car- und Car-to-Infrastructure-Kommunikation verbreiten sich immer mehr. Mit einer Mikrosimulation des Automarkts wurde bestimmt, welche Modelle sich durchsetzen: Eine virtuelle Käuferpopulation entscheidet dabei anhand der Spezifikationen der Fahrzeuge und dem in der Realität beobachteten Kaufverhalten der Konsumenten, welchen Autos der Vorzug gegeben wird. So schrecken zum Beispiel im BAU- und EFF-Szenario die Ein-AutoHaushalte vor dem Kauf von Elektroautos aufgrund der Reichweitenlimitation zurück; im COM-Szenario gilt diese Einschränkung nicht, da hier von einer Änderung des Mobilitätsverhaltens hin zu mehr Intermodalität und mehr gemischten Wegeketten von motorisiertem individuellem Verkehr (MIV) und öffentlichem Verkehr (ÖV) ausgegangen wird, z.B. dank neuen IT-Applikationen mit Echtzeitplanung. Das heisst, man wechselt viel einfacher vom universellen Verbrennungsmotorfahrzeug auf ein in Reichweite und Funktionalität limitiertes Elektrofahrzeug. Dadurch nimmt die Elektromobilität im COM-Szenario am schnellsten zu.

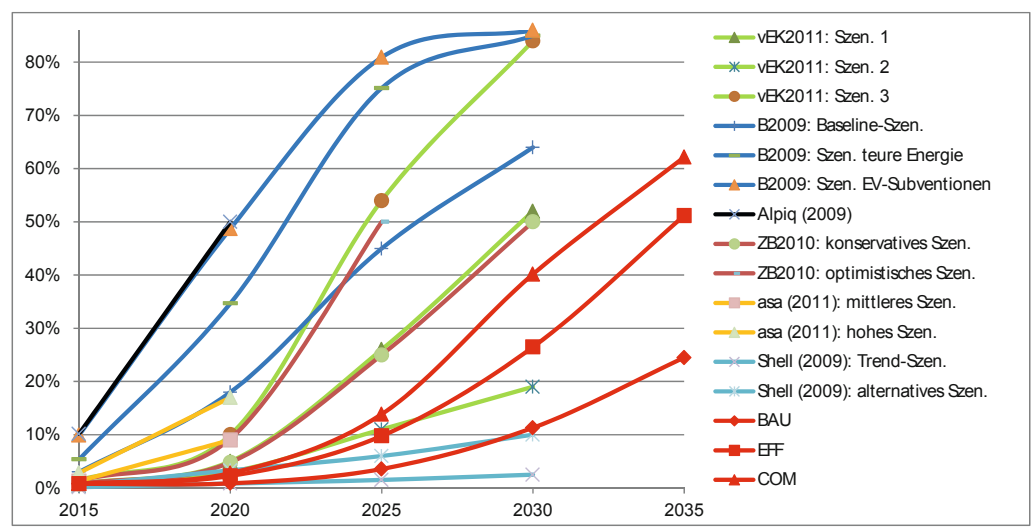

Abbildung 5: Schätzungen zum Anteil der Elektrofahrzeuge am Neuwagenmarkt in der EU (van Essen und Kampmann), den USA (Becker et al.), Deutschland (Shell) und in der Schweiz (alpiq; Zah und Binder; asa); rot eingezeichnet die Ergebnisse für die drei Szenarien BAU, EFF und COM der vorliegenden Studie. 
Gemäss den Szenarien wird die Zukunft im Verkehr ganz generell eine Reduktion der Treibhausgasemissionen mit sich bringen. So resultiert bei allen Szenarien, also auch bei einer Fortschreibung der derzeitigen Entwicklung bis zum Jahr 2020 eine praktisch identische Reduktion der Treibhausgasemissionen aus dem Verkehr von 10 Prozent gegenüber heute und dies, obwohl mit einer Mobilitätsausweitung von 24 Prozent gerechnet wird. Auch im Jahre 2035 beträgt die Reduktion des Elektromobilitätsszenarios (COM) gegenüber dem BAU-Szenario erst 17 Prozent. Erst im Jahr 2050 sind es dann über 30 Prozent.

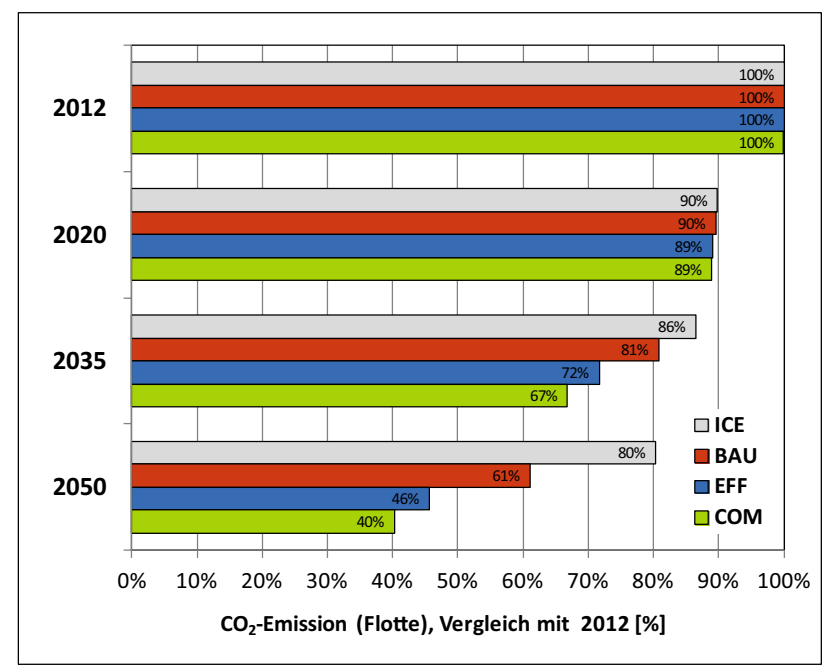

Abbildung 6: Gesamthafte Reduktion der Treibhausgasemissionen in den Szenarien BAU, EFF und COM und einer ausschliesslichen Optimierung von Verbrennungsfahrzeugen (ICE) gegenüber dem Jahr 2012.

Auch im Business-as-Usual-Szenario, bei dem einzig die heutigen Trends fortgeschrieben werden und keine zusätzliche Steuerung in Richtung Energieeffizienz (EFF-Szenario) oder Elektrifizierung (COM-Szenario) erfolgt, findet eine gewisse Elektrifizierung der Flotten statt. Auch für das BAU-Szenario gelten die $\mathrm{CO}_{2}$-Flottenziele der EU und der Schweiz (siehe Abbildung 6).

Selbst wenn auch in der Schweiz in zwei Jahrzehnten laut der derzeitigen Energiestrategie des Bundes die Stromerzeugung aus Gaskraftwerken mit einer entsprechenden Freisetzung von Treibhausgasen ein vorübergehendes Maximum erreicht, muss nicht mit einer deutlichen Verschlechterung der Umweltbilanz im 
COM-Szenario gerechnet werden (siehe Abbildung 7). Abschätzungen dieser Studie zeigen, dass die für die Elektromobilität notwendigen Strommengen zumindest in den nächsten zwei Jahrzehnten unterhalb der vom Bund in seiner Planung dafür eingesetzten Mengen liegen (siehe Kapitel 9.1.3). Damit konnte in dieser Untersuchung mit den aus den Planungsstudien des Bundes ersichtlichen Emissionsdaten für die zur Verfügung stehende Elektrizität gerechnet werden.

\section{Vorteile der Elektromobilität}

Insgesamt zeigt der Vergleich der drei Szenarien, dass sowohl eine Förderung der Effizienz generell (EFF) als auch eine gezielte Förderung der Elektromobilität (COM) gegenüber dem Business-as-Usual-Szenario leicht vorteilhaft ist. Positiv zugunsten des Effizienz- (EFF) und des Elektromobilitätsszenarios (COM) fällt insbesondere die Verminderung von Treibhausgasen ins Gewicht. Bis im Jahr 2050 würde sich der Ausstoss von Kohlendioxidäquivalent in den Förderszenarien gegenüber einer Business-as-Usual-Entwicklung deutlich reduziert haben (-25 Prozent bei EFF, -34 Prozent bei COM).

Vorteile weisen Elektrofahrzeuge im Vergleich zu Verbrennungsmotorautos auch im Bereich Umwelt auf (Kapitel 5). Da sind es vor allem Luft- und Lärmbelastung, die bei Elektrofahrzeugen etwas tiefer liegen als bei Verbrennungsmotoren. Was das Nachhaltigkeitsziel der Minimierung des Verlustes von metallischen Rohstoffen angeht, ist die Tendenz bei beiden ähnlich ungewiss. Bei den wirtschaftlichen Aspekten fällt die Bilanz beim Ziel «Investitions- und Betriebskosten für Infrastruktur minimieren» bei der Elektromobilität schlechter aus als bei den fossilbetriebenen Autos. Bei beiden ist mit höheren Investitions- und Betriebskosten für die Fahrzeuge zu rechnen und es gilt die Verfügbarkeit der verschiedenen Rohstoffe im Auge zu behalten. Wirtschaftlich leicht positiver bewertet wird das Elektromobilitätsszenario dagegen unter Punkten wie «Absatzmärkte für Schweizer Unternehmen schaffen» oder «Flexibilität des Systems erhöhen». Markant besser schneidet es zudem beim Bestreben ab, die Abhängigkeit von einzelnen Energieträgern zu senken. Die Möglichkeit, erneuerbare Energien für den Verkehr zu nutzen, ist denn auch einer der wichtigen Pluspunkte. Auch bei der Erhöhung der Sicherheit im Strassenverkehr, die im gesellschaftlichen Bewertungsteil diskutiert wird, steht die Elektromobilität leicht besser da als konventionelle Fahrzeuge, weil der gegenwärtige eSafety-Trend, der vor allem kleineren und leichteren Fahrzeugen mehr Sicherheit bringt, durch eine Ausweitung der Elektromobilität verstärkt werden dürfte. Spezifische Probleme der Elektromobilität im Zusammenhang mit Reboundeffekten zeigen sich in der Untersuchung dagegen keine. 


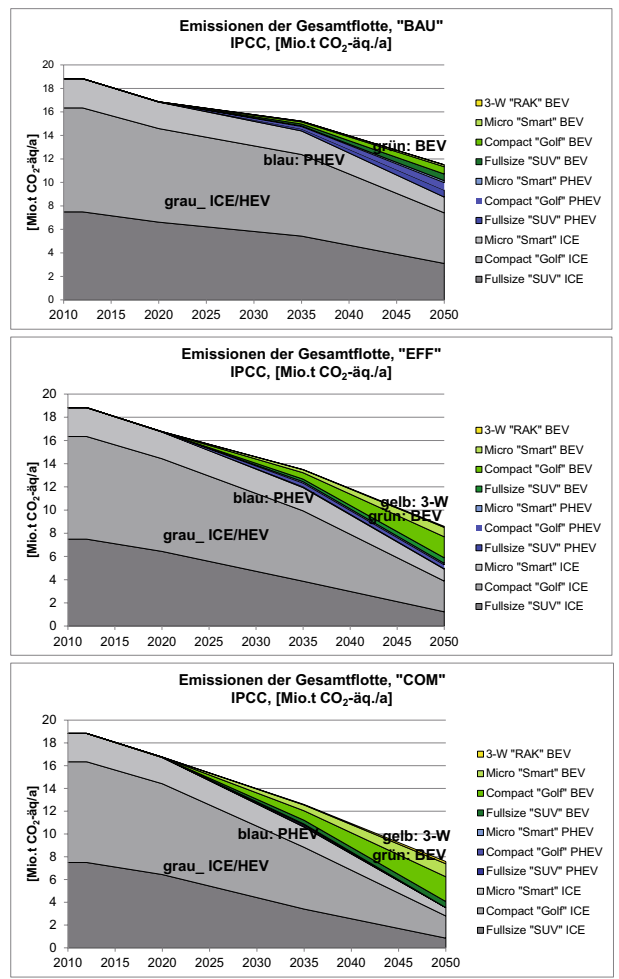

Abbildung 7: $\quad$ Gesamter $\mathrm{CO}_{2}$-Ausstoss des motorisierten Individualverkehrs in der Schweiz.

Die Unterschiede zwischen den beiden Szenarien EFF und COM sind relativ klein. Dies, weil eine generell stringente Effizienzpolitik nicht nur vielfach Elektromobile begünstigt, sondern auch, weil hier die fossil getriebenen Fahrzeuge gezwungenermassen auch effizienter werden. Innerhalb dieser beiden Szenarien sind die Unterschiede bei der Bewertung entsprechend auch bei den anderen Faktoren gering. Bei vielen Bewertungspunkten kommt das COM-Szenario dennoch besser weg als das EFF-Szenario. Ein wichtiger Trend im COM-Szenario ist die Verlagerung hin zu kleineren Fahrzeugen - so ergibt die Modellierung der Flottenentwicklung für das COM-Szenario bis 205010 Prozent Anteil an ultraleichten Mikrofahrzeugen (3-Wheeler wie Renault Twizy). Diese neue Fahrzeugkategorie für den urbanen Bereich wird durch einen elektrischen Antrieb erst ermöglicht und erlaubt eine Dematerialisierung der Mobilität. 


\section{Nachteile der Elektromobilität}

Negativ zu Buche schlagen dagegen bei der Elektromobilität neben den erwähnten höheren Investitionskosten auch der höhere Ressourcenverbrauch bei der Herstellung der Fahrzeuge. Dies führt generell zur Verlagerung der Umweltbelastungen aus der Nutzphase in die Vorketten. Dadurch wird auch die Messung - und damit die gesetzliche Regulierung der Umweltbelastungen - erschwert. Noch vordringlicher ist jedoch die Feststellung, dass im derzeitigen Steuersystem die Einnahmen aus der Mineralölsteuer bei einer Ausweitung der Elektromobilität markant schrumpfen. Je schneller sich Elektrofahrzeuge - und generell effizientere Autos - durchsetzen, umso rascher brechen die Steuereinnahmen ein.

\section{Empfehlungen}

Eine der wichtigsten Empfehlungen lautet denn auch, möglichst bald den Übergang von der Besteuerung der fossilen Treibstoffe auf eine kilometerabhängige Besteuerung einzuleiten (siehe Kapitel 8.3 und 9.3). Angesichts der insgesamt geringen Differenzen zwischen dem Effizienz- und dem Elektromobilitätsszenario drängen sich im Übrigen keine technologiespezifischen Anreize zur Förderung der Elektromobilität auf. Würden solche ergriffen, bestünde angesichts der kleinen Unterschiede und der Unsicherheiten, die Szenarien immer mit sich bringen, vielmehr die Gefahr, ineffiziente Entwicklungen zu unterstützen. Zu empfehlen sind jedoch technologieneutrale Massnahmen wie Mindestvorschriften, Maximalwerte sowie Mittelwerte für den Treibhausgasausstoss der Personenwagenneuzulassungen zur Erhöhung ihrer Gesamteffizienz und Limitierung des gesamten Energieverbrauchs. Damit wird indirekt auch die Elektromobilität gefördert. Die Studie listet eine ganze Reihe von einzelnen Schritten auf, die der Bund, die Kantone, die Gemeinden, die Autobranche, die Elektrizitätswirtschaft und interessierte Verbände angehen können und sollten (Kapitel 9.4). 


\section{Aufbau des vorliegenden Berichts}

In der Einleitung (Kapitel 1) werden die Ausgangslage, die Motivation und die Fragestellung der Studie erläutert, zudem finden sich darin Literaturanalyse, Begriffsdefinitionen sowie der Beschrieb der Gesamtmethodik (Peter de Haan und Rainer Zah).

Zu Beginn der Studie werden die drei Szenarien erarbeitet (Kapitel 2), welche sich einerseits in die bestehenden Grundlagenarbeiten der Bundesämter (Bevölkerungsperspektiven des BFS, Verkehrsperspektiven des ARE, Energieperspektiven des BFE) einordnen und anderseits die Breite der möglichen künftigen Entwicklung aufspannen und die nachfolgenden Auswirkungen auf Umwelt, Wirtschaft und Gesellschaft aufzeigen (Peter de Haan und Daniel Arnet).

Daran schliesst die Analyse der Fahrzeugkomponenten (Kapitel 3) an, die untersucht, welche relevanten Technologien bei Batterien, Motoren, Leistungselektronik und Ladegeräten schon am Markt erhältlich sind und wie sich die Technologie im Untersuchungszeitraum weiterentwickeln könnte (Rainer Zah und Marcel Gauch).

Basierend auf den Trends bei den Einzelkomponenten wird darauf die zukünftige Entwicklung der Fahrzeuge analysiert (Kapitel 4). Hierbei werden für typische Fahrzeugkategorien und unterschiedliche Antriebssysteme (konventionell, elektrisch, plug-in-hybrid), Energiebedarf, Materialzusammensetzung und Gewicht abgeschätzt (Rainer Zah und Marcel Gauch).

Es folgt die Analyse der Umweltauswirkungen von Elektromobilität mittels der Lebenszyklusanalyse (Kapitel 5). Im Mittelpunkt stehen Energieeffizienz, Treibhausgasemissionen und weitere Umweltauswirkungen heutiger und zukünftiger Elektromobilität aus der Lebenszyklusperspektive: Produktion, Nutzung und Entsorgung von Fahrzeugen und Infrastruktur sowie Energiebereitstellung (Rainer Zah, Marcel Gauch und Hans-Jörg Althaus).

Die Lebenszyklusanalyse kann systemische Aspekte wie zum Beispiel Fragen der Kritikalität von Rohstoffen nicht abdecken. In einer separaten Betrachtung, welche auch die Nachfrage nach kritischen Rohstoffen für Nicht-Transport-Anwendungen abdeckt (Kapitel 6), werden Verfügbarkeit und Nutzungskonkurrenz 
von kritischen Metallen beurteilt; es wird dabei zwischen geologischer, ökonomischer, technischer, geopolitischer und ökologischer Knappheit differenziert (Patrick Wäger, Denise Fussen und Rolf Widmer).

Auch volkswirtschaftliche und gesellschaftliche Auswirkungen werden durch die Lebenszyklusanalyse nicht abgedeckt. Die sozioökonomischen Folgen von Elektromobilität für die Schweiz werden analysiert, so beispielsweise mögliche Konsequenzen auf den Steuerhaushalt und Infrastrukturausgaben der öffentlichen Hand (Kapitel 7) (Denise Fussen, Katrin Bernath und Peter de Haan).

Die Lebenszyklusanalyse betrachtet die Umweltbelastung pro zurückgelegten Fahrzeugkilometer; sie kann dabei verschiedene Fahrzeuggrössen unterscheiden und die unterschiedliche Flottenzusammensetzung je Szenario berücksichtigen. Um die Resultate einander gegenüberstellen zu können, ist die angenommene Verkehrsleistung in jedem der drei Szenarien aber gleich. Aber könnten energieeffizientere Fahrzeuge, und insbesondere Elektromobile, zu Mehrverkehr führen? Kapitel 8 untersucht, aus welchen Gründen solche sogenannten Reboundeffekte auftreten könnten und wie ihnen entgegengewirkt werden kann (Peter de Haan).

In der anschliessenden Gesamtbeurteilung (Kapitel 9) werden Bilder zukünftiger Elektromobilitätsszenarien in der Schweiz gezeichnet und ihre Konsequenzen diskutiert. Im Mittelpunkt steht die Frage, wie das zukünftige Verkehrs- und Transportsystem der Schweiz konzipiert sein müsste, damit Elektrofahrzeuge ihre Stärken zugunsten einer nachhaltigen Mobilität ausspielen können. Diese Gesamtbetrachtung schliesst die Bereitstellung von Elektrizität mit ein und berücksichtigt auch das Vehicle-to-Grid-Potenzial und die allfällige Nutzung von ausgemusterten Fahrzeugbatterien in stationären Anwendungen. Die Studie schliesst mit konkreten Empfehlungen für eine nachhaltige Nutzung der Elektromobilität (Peter de Haan \& Rainer Zah). 


\section{Danksagung}

Dieses Buch hätte nicht entstehen können ohne die mehrfache kritische Begutachtung durch die Mitglieder der Begleitgruppe, denen wir hiermit herzlich für ihre Teilnahme und ihre teils ausführlichen Kommentare danken: Jörg Fromm (Präsident der Begleitgruppe), Heidi Blattmann, Christian Bühlmann, Lino Guzzella, Stefan Hirschberg, Kurt Hug, Christoph Jahn, Mario Keller, Dieter Kraft, Cornelia Moser, Tobias Ott, Felix Reutimann, Martin Schiess, Fridolin Stähli, Daniel Wachter und Michael Weber.

Ebenso danken wir Sergio Bellucci und Lucienne Rey vom Schweizerischen Zentrum für Technologiefolgenabschätzung (TA-SWISS) für die kooperative Projektleitung, und den Bundesämtern für Energie (BFE), Raumplanung (ARE), Strassen (ASTRA) und Umwelt (BAFU) sowie dem Basler Fonds der Familie Ernst Basler für die Unterstützung des Projekts.

Für ausführliche Fachgespräche, wertvolle Hinweise und schriftliche Ergänzungen bedanken wir uns ferner bei Christian Bach, Roland Frei, Lorenz Raymann und Patrik Soltic sowie bei Daniel Arnet, der seine Masterarbeit an der ETH Zürich im Rahmen dieser Studie durchführte. Ein besonderer Dank geht an Heidi Blattmann und Christine D’Anna-Huber für kommunikative Unterstützung sowie die Schlussredaktion des Manuskripts.

Diese Arbeit beruht zu grossen Teilen auf der Ökobilanzierung von Elektromobilität, welche zusammen von Empa und dem Paul Scherrer Institut (PSI) im Rahmen des Projekts THELMA (Technology centered electric mobility assessment) durchgeführt und vom Kompetenzzentrum des ETH-Bereichs für Energie und Mobilität (CCEM), der Schweizer Erdölvereinigung und von Swiss Electric Research kofinanziert wurde. 


\section{Bedeutung von Elektrofahrzeugen im Kontext unserer Mobilität und des Energiesystems}

\section{$1.1 \quad$ Einleitung}

\subsubsection{Warum eine TA-SWISS-Studie zur Elektromobilität?}

Der «ökologische Fussabdruck» pro Kopf ist in den Industrieländern zu gross, als dass weitere 1 bis 1,5 Milliarden Einwohner von Schwellenländern ein ähnliches Wohlstandsniveau anstreben könnten, ohne dass dazu auf Kosten künftiger Generationen Ressourcen benötigt würden. Von diesem allgemeinen Umweltwissen hin zu einer Verhaltensabsicht und schliesslich zu tatsächlichen Verhaltensänderungen ist ein weiter Weg. Diese Problematik akzentuiert sich in der Vergangenheit wie in der Zukunft - bei der individuellen motorisierten Mobilität, wo die Kosten und Auswirkungen des Gesamtsystems hoch sind, der Nutzen aber beim Einzelnen anfällt und der Einfluss einer individuellen Verhaltensänderung scheinbar gering ist.

Die Elektromobilität erscheint in diesem Zusammenhang als vielversprechende Zukunftstechnologie. Effizient und lokal emissionsfrei soll sie uns eine gegenüber heute nochmals individuellere und flexiblere Mobilität ermöglichen. Entsprechend ist das Thema in den Medien ein Dauerbrenner. Längerfristig soll dadurch die auf fossile Energie basierte Mobilität abgelöst werden, zumindest im Nahverkehr. Bei näherer Betrachtung zeigen sich allerdings Knackpunkte, welche für die Gesamtbilanz der Elektromobilität entscheidend sind. Analogien finden sich bei Biotreibstoffen (wo auf anfängliche Euphorie nach einer umfassenden Technikfolgenabschätzung Ernüchterung folgte) und bei der Vision einer Wasserstoffgesellschaft. Die systematische Aufarbeitung der Technikfolgen erlaubt, Politikziele und -instrumente so zu wählen, dass eine nachhaltige Entwicklung möglich wird.

Die Elektromobilität steht auf der Schwelle zum Marktdurchbruch. Eine Vielzahl an technischen Grundlagen und Zukunftsprognosen stehen zur Verfügung. Erst das Zusammenwirken vielfältiger Systemkomponenten und Faktoren entscheidet jedoch darüber, in welchen Mobilitätssegmenten die Elektromobilität insge- 
samt für das Mobilitäts- und Energiesystem sowie für die Gesellschaft Vorteile bringt. Um dies zu beurteilen, bietet sich eine umfassende Technologiefolgenabschätzung von Elektromobilität an.

\subsubsection{Bedeutung der Thematik}

Nationale Bedeutung: Die Relevanz der Elektromobilität ist vor allem bei der Klimadebatte, der lokalen Luftreinhaltung, der künftigen Stromproduktion in der Schweiz (Bandenergie vs. lokale stochastische Produktion) und der Clean-TechStrategie gegeben. Innerhalb der Erarbeitung der Energiestrategie 2050 des Bundesrates stellt sich ebenfalls die Frage, wie schnell die Marktdurchdringung der Elektromobilität erfolgen kann, welche Vor- und Nachteile sich dabei einstellen und welche Instrumente eingesetzt werden sollen, um die Vorteile zu sichern und die Nachteile zu mindern.

Internationale Bedeutung: Die Elektromobilität ist eine der Schlüsseltechnologien zur Reduktion der Abhängigkeit von fossilen Energiequellen sowie zur Erreichung der Klimaschutzziele vieler Industriestaaten. Für die Automobilindustrie erfordert sie neue Technologien, neue Geschäftsmodelle sowie die Sicherung des Nachschubs an seltenen, nicht erneuerbaren Ressourcen.

Technologische Bedeutung: Mit Elektromobilität verlagern sich fossile Versorgungsketten hin zum Strom, der Trend zu Elektrizität als «Ausgleichswährung» zwischen den Energieträgern verstärkt sich. Das bis anhin losgelöste Strassenverkehrenergiesystem wird mit dem übrigen Energiesystem verknüpft, und zwar bidirektional auf den untersten Netzebenen.

Wirtschaftliche Bedeutung: Elektromobilität beschleunigt den ohnehin stattfindenden, aus dem ICT-Sektor bekannten Trend, dass die Wertschöpfung weniger im Gerät als in der Mobilitätsdienstleistung liegt. Klassischer Automobilbau wird in dem Sinne an Bedeutung verlieren zugunsten neuer Geschäftsmodelle, und bei der Elektromobilität sind neue Schlüsseltechnologien (Batterien, Elektromotoren, Hochleistungselektronik) wichtig. Dies stellt ein Risiko dar für etablierte Automobil- und Motorenbauer, bietet aber neue Chancen für Zulieferer - auch für innovative KMU in der Schweiz.

Politische Bedeutung: Viele der potenziell positiven systemischen Effekte der Elektromobilität, insbesondere die Interaktion mit der dezentralen stochastischen 
Stromproduktion, erfordern das frühzeitige Setzen von Anreizen, Vorschriften und Normen sowie regulatorische Eingriffe namentlich im Elektrizitätssystem, um neue Geschäftsmodelle zu ermöglichen.

Gesellschaftliche Bedeutung: Die Elektromobilität verstärkt den Trend weg vom universellen Fahrzeug («Renn-Reise-Limousine») hin zu einer Ausdifferenzierung der Fahrzeugnutzung (Nahverkehr, Langstrecke usw.). Die Elektromobilität kann auch eine Zunahme der Mobilität insgesamt oder die Verlagerung von anderen Verkehrsträgern (wie Langsamverkehr und öffentlicher Verkehr) hin zu Elektromobilität induzieren (sogenannter Reboundeffekt).

\subsubsection{Systemabgrenzung}

Die vorliegende Studie beschreibt die positiven und negativen Eigenschaften von Elektrofahrzeugen bei ihrer Produktion, im Betrieb und in der Entsorgung. Sie konzentriert sich auf Elektromobilität in der Schweiz und fokussiert auf den individuellen motorisierten Personentransport. Um eine umfassende und in sich kohärente Lebenszyklusbetrachtung zu gewährleisten, werden jedoch auch wichtige Prozesse ausserhalb der Schweiz (Rohstoffe, Fahrzeugherstellung, Energiebereitstellung, Entsorgung), ausserhalb des Personentransports (Wechselwirkungen mit dem Strassengüterverkehr und dessen Energiesystem) und ausserhalb der individuellen Mobilität (Abhängigkeit von sowie Kombinationsmöglichkeiten mit dem öffentlichen Verkehr) betrachtet.

Elektromobilität wird für die vorliegende Studie (siehe Kapitel 1.4) definiert als individuelle Mobilität, die auf Fahrzeugen basiert, welche hauptsächlich von einem Elektromotor angetrieben werden und den Strom hauptsächlich aus stationären Quellen beziehen. Explizit werden auch als «Elektroauto» angekündigte dreirädrige Fahrzeugkonzepte betrachtet, welche rein zulassungsrechtlich Motorräder sind.

\subsubsection{Ziele der vorliegenden Technikfolgenabschätzung zur Elektromo- bilität: sechs Leitfragen}

Für den Zeitraum bis 2035/2050 werden mögliche Entwicklungspfade der Elektromobilität in der Schweiz in drei Szenarien skizziert und deren Chancen und Risiken für Umwelt, Wirtschaft und Gesellschaft beleuchtet. Die komplexen Zusam- 
menhänge der Technologieentwicklung und Marktpenetration werden analysiert und Einflussmöglichkeiten sowie deren Wirkungen aufgezeigt. Die Technologiefolgenabschätzung richtet sich an den Lebenszyklusphasen (Produktion, Betrieb, Entsorgung) aus und thematisiert insbesondere die folgenden sechs Leitfragen:

- Wie könnten sich die unterschiedlichen Formen von Elektrofahrzeugen weiterentwickeln? Welche Marktpenetrationsverläufe und Marktanteile sind minimal und maximal möglich?

- Wie wichtig ist die Rolle seltener Materialien für die Produktion von Elektrofahrzeugen (Batterien und Permanentmagnete)? Könnte es für andere Technologiezweige zu Knappheit kommen?

- Wie viel Strom wird zusätzlich benötigt? Wie können Elektrofahrzeuge den grösstmöglichen positiven Nutzen erbringen?

- Gibt es spezifischen Handlungsbedarf für die nachhaltige Entsorgung von Elektrofahrzeugen und das Schliessen der Stoffkreisläufe?

- Sind Reboundeffekte zu erwarten und lassen sich diese vermeiden? Wann müssten Einnahmenausfälle der öffentlichen Hand durch neue Steuersubstrate kompensiert werden?

- Sollen allgemein energieeffiziente Fahrzeuge gefördert werden oder spezifisch Elektrofahrzeuge?

\subsection{Zusammenhänge}

Die Elektromobilität erscheint in erster Linie als Ersatz fossiler Energieträger für die Fortbewegung eines Autos. Damit kommt aber just jener Energieträger ins Spiel, der als Rückgrat des gesamten künftigen Energiesystems zu betrachten ist und als "Ausgleichswährung» zwischen allen anderen Energieträgern funktioniert. Damit beeinflusst die Elektromobilität auch das Gesamtenergiesystem. Elektroautos beenden die auf Benzin und Diesel basierende «getrennte Energiewelt» der Mobilität. Zudem kann die Elektromobilität sich in unserem heutigen Mobilitätssystem nicht nur einen Platz sichern, sondern durch Rückkopplung dieses Mobilitätssystem auch wieder beeinflussen. Im Folgenden greifen wir einige dieser Aspekte qualitativ auf, auch wenn sie im quantitativen Teil der Studie, d.h. bei der Bewertung aufgrund einer Lebenszyklusanalyse, nicht explizit berücksichtigt werden können:

- Elektromobile können Teil eines lokalen Energiesystems mit einem gewissen Autarkiegrad sein. Der Strom von Fotovoltaikanlagen auf dem Dach kann in 
der Batterie dann eingespeichert werden, wenn sie anfällt. Fahrzeuge stehen durchschnittlich 23 von 24 Stunden täglich (wenn auch teilweise am Arbeitsplatz und nicht zu Hause). Wenn diese Standzeiten genutzt werden, um die Batterien zu laden, lässt sich namentlich die Belastung der unteren Netzebene durch lokale Einspeisung reduzieren bzw. das Erfordernis des Abwerfens lokaler Einspeisung im Falle von Netzinstabilitäten (oder eventuell auch zur Erhaltung der Netzstabilität) vermindern. In Deutschland wird der Eigenverbrauch von Fotovoltaikstrom aus diesen Gründen bereits im Rahmen der EEG gefördert. Mit der Energiestrategie 2050 formuliert der Bundesrat das Ziel, eine entsprechende Eigenverbrauchsregelung (Net Metering) auch in der Schweiz einzuführen (Bundesrat 2012).

- Auch eine Zusammenfassung von Elektrofahrzeugen zu einem virtuellen Regelleistungskraftwerk ist denkbar, eine entsprechende zentral gesteuerte Kommunikation und den jederzeitigen Zugriff auf die meisten Fahrzeuge vorausgesetzt, vor allem in Form negativer Regelleistung (Unterbrechen der Ladevorgänge). Dies kann den Zubaubedarf an Regelleistung reduzieren. Bei Vorliegen entsprechender Geschäftsmodelle ist sogar eine Verbilligung der Elektromobilität denkbar, zum Beispiel über reduzierte Batteriemietgebühren. Allerdings ist aus heutiger Sicht eher zu erwarten, dass die Anstrengungen sich auf «Smart Charge» beschränken und Rückspeisung von Elektrofahrzeugbatterien ins Netz nur in Einzelfällen stattfindet und stattdessen die weitere (stationäre) Verwendung älterer Batterien («2nd Life») in den Vordergrund tritt.

- Elektromobilität kann auch zu einer Förderung der Intermodalität beitragen, und umgekehrt würde die Elektromobilität von Fortschritten bei der Verknüpfung von motorisiertem Individualverkehr (MIV) und öffentlichem Verkehr (ÖV) profitieren, namentlich durch Echtzeitinformationssysteme. Im Rahmen der vorliegenden Studie gehen wir im dritten der betrachteten Szenarien von einem solchen stärker vernetzten Mobilitätssystem aus, bei dem Leute häufiger umsteigen und spezifische Fahrzeugtypen daher gezielter verwenden. Wir belassen die Gesamtzahl der Fahrzeugkilometer jedoch konstant und ändern nur die Fahrzeugzusammensetzung, um den Vergleich zwischen den Szenarien nicht zu erschweren.

- Gerade elektrische Zwei- und Dreiräder können einen wesentlichen Einfluss auf unser heutiges Mobilitätssystem haben. Sogenannte «Pedelecs» werden namentlich für Freizeit- und Arbeitswege als sehr gut geeignet einge- 
stuft. Sie können das Radfahren auch insgesamt erleichtern, erlauben die Bewältigung grösserer Distanzen und schwieriger Topografie, tragen zu einer Verminderung von gesundheitlichen Problemen bei und können sich als Ersatz von Autofahrten anbieten (Le Bris 2011; Lanzendorf und Prill 2011).

\subsection{Relevante Aspekte}

Es existiert bereits eine Vielzahl von Studien zur Energieeffizienz und den Treibhausgasemissionen von Elektromobilität. Ebenso finden sich viele Prognosen zur zukünftigen Rolle von Elektromobilität in der Gesellschaft. Oftmals werden aber nur Einzelaspekte betrachtet und nicht der gesamte Lebenszyklus von der Herstellung (sowohl der Fahrzeuge wie auch der Energie) über den Betrieb bis zur Entsorgung. Diese partielle Betrachtungsweise verleitet oft zu Schlüssen, welche aus der Gesamtschau heraus nicht unterstützt werden können.

Die Umweltauswirkungen des Strassentransports manifestieren sich in mehreren Umweltsystemen (Giannouli et al. 2006). Der Betrieb von Elektrofahrzeugen wurde in diversen Well-to-Wheel- (WTW) und Life-Cycle-Assessment-Studien (LCA; ISO 14‘040 2006; ISO 14‘044 2006) untersucht (für eine Übersicht siehe Althaus, Gauch 2010). WTW-Studien beschränken sich typischerweise auf die Betriebsphase und vernachlässigen die benötigte Infrastruktur. Meist fokussieren sie auch auf Energiebedarf und Treibhausgasemissionen (Carbon Footprint). Im Gegensatz dazu berücksichtigen LCA-Studien auch die Herstellung und Entsorgung der Fahrzeuge und teilweise auch der Strasseninfrastruktur. Auch weisen sie meist zusätzliche Indikatoren aus, zum Beispiel bezüglich Gesundheits-, Ökosystem- oder Ressourcenschäden. Diese Studien zeigen, dass sich die Herstellung eines Elektrofahrzeuges signifikant unterscheidet von der Herstellung eines Fahrzeuges mit einem Verbrennungsmotor, und schliessen daraus, dass die Berücksichtigung der Fahrzeuginfrastruktur ein Muss für einen sinnvollen Vergleich ist. Gezeigt wurde auch, dass die Produktion und Entsorgung von Elektrofahrzeugen einen relevanten Teil der Umweltbelastung im Lebenszyklus ausmachen kann, sofern der Strom zum Betrieb der Fahrzeuge mit umweltfreundlichen Technologien erzeugt wird (Althaus und Gauch 2010; Bauer und Simons 2010; Notter et al. 2010). Bei einer fossilen Stromerzeugung hingegen dominiert oftmals die Betriebsphase die Lebenszykluseffekte des Fahrzeuges.

Ressourcenfragen werden oft ausserhalb von Lebenszyklusbetrachtungen diskutiert. Dabei geht es insbesondere auch darum, ob die durch Zukunftstechnologien induzierte Nachfrage nach Ressourcen nachhaltig gedeckt werden kann. 
Wie verschiedene, kürzlich erschienene Studien gezeigt haben, stellt sich diese Frage in besonderem Masse für die sogenannten geologisch seltenen Metalle (d.h. Metalle, welche in durchschnittlichen Konzentrationen <0.01 Prozent in der Erdkruste vorkommen), welche nach heutigem Stand des Wissens bei verschiedenen Zukunftstechnologien eine wesentliche Rolle spielen werden und deren Versorgungslage teilweise als kritisch eingestuft wird (siehe z.B. Andersson, Rade 1999; Andersson 2001; Andersson, Rade 2001; Angerer et al. 2009a; European Commission 2010; National Research Council 2008; U.S. DOE 2011; Wäger et al. 2010). Für die Elektromobilität von Bedeutung sind in diesem Zusammenhang etwa Kupfer (Batterien, Leitungen), Lithium (Batterien), Platingruppenmetalle (Elektronik) oder seltene Erden wie Cer (Batterien), Dysprosium (Permanentmagnete), Lanthan (Batterien), Neodym (Batterien, Permanentmagnete), Praseodym (Batterien, Permanentmagnete) oder Samarium (Permanentmagnete) (U.S. DOE, 2011). Eine nachhaltige Versorgung mit diesen Metallen wird nicht zuletzt auch davon abhängen, ob es gelingt, sie aus Produktionsabfällen und ausgedienten End-of-Life-Produkten EoL zurückzugewinnen (Wäger et al. 2011a).

Gesellschaftlich relevant ist namentlich die Verkehrssicherheit. Die Lautlosigkeit von Elektrofahrzeugen wird kritisch diskutiert, hier dürfte es sich jedoch eher um vorübergehende Gewöhnungs- und Regulierungseffekte handeln. Bei den Zweirädern führt die Elektromobilität jedoch zu höheren mittleren Geschwindigkeiten und einer Zunahme entsprechender Unfallbilder, was bereits erste regulatorische Massnahmen (Unterteilung in Kategorien nach Maximalgeschwindigkeit; Einstufung als Motorfahrräder; Helmpflicht) erfordert hat.

Bei den ökonomischen Effekten steht der höhere inländische Anteil an der Wertschöpfungskette ebenso wie die Stärkung forschungsintensiver Industriezweige im Vordergrund. Die Reduktion der Abhängigkeit von importierter fossiler Energie und des damit einhergehenden Kapitalexports ist in der Regel volkswirtschaftlich positiv (siehe z.B. McKinsey 2009). Jedoch stellt die Umlagerung der Steuersubstrate weg von der Mineralölsteuer eine Herausforderung für den Fiskus dar, die Elektromobilität wird deshalb als Wegbereiter für Road-Pricing-Ansätze zur Finanzierung von Unterhalt und punktuellem Ausbau des Strassennetzes gesehen.

\subsection{Allgemeine Definitionen}

In ihrer breitesten Bedeutung umfasst die Elektromobilität den Güter- oder Personentransport mit Fahrzeugen, die durch Elektromotoren angetrieben werden. 
Dies beinhaltet Züge, Trams und Trolleybusse sowie Individualverkehrsmittel, die den Strom für ihren Antrieb aus Batterien, Kondensatoren, Brennstoffzellen oder sonstigen Generatoren beziehen. Für die vorliegende Studie gilt das Interesse der Individualmobilität. In diesem Bereich sind heute vor allem Fahrzeuge im Gespräch, welche die elektrische Energie in Batterien speichern. Denkbar sind aber auch Varianten, die ihren Strom aus Kondensatoren beziehen. Elektrofahrzeuge können die elektrische Energie von stationären Quellen (im Allgemeinen aus dem Stromnetz) beziehen.

Entsprechend definieren wir hier Elektromobilität als individuelle Mobilität, die auf Fahrzeugen basiert, welche (i) hauptsächlich von einem Elektromotor angetrieben werden und (ii) Elektrizität hauptsächlich aus stationären Quellen beziehen. Im Folgenden gehen wir auf mehrere Fahrzeugarten und Antriebskonzepte ein, um die Grenzen der Definition zu verdeutlichen.

Zwei- und Dreiräder: Elektrische Zweiräder gehören zur Elektromobilität, sobald Elektrizität gegenüber der Muskelkraft die grössere Bedeutung zukommt. Elektrofahrräder gehören also eher nicht dazu (Tretunterstützung, der Fahrer muss immer treten), Elektromotorräder (inkl. Scooter) aber schon. Erwähnenswert sind auch mehrere als «Elektroauto» angekündigte dreirädrige Fahrzeugkonzepte, welche rein zulassungsrechtlich als Motorräder und nicht als Personenwagen gelten.

Brennstoffzellenfahrzeuge: Brennstoffzellenfahrzeuge (fuel cell vehicle, FCV) werden oft auch als elektrische Fahrzeuge verstanden. Der Energieträger ist hier Wasserstoff, Elektrizität wird in der Brennstoffzelle produziert und in einer Batterie zwischengespeichert, bevor sie in Bewegungsenergie umgesetzt wird. Die Batterie ist nötig, da eine Brennstoffzelle die Leistung nicht schnell genug regulieren kann, um ein Auto zu beschleunigen. In diesem Sinne sind Brennstoffzellenfahrzeuge nach unserer Definition keine Elektrofahrzeuge, sondern (Hybrid-) Wasserstofffahrzeuge (so wie eine dieselelektrische Lokomotive als Diesellokomotive verstanden wird, auch wenn ein dieselbetriebener Generator Strom produziert und damit ein Elektromotor betrieben wird). Wasserstoff wird heute aus Erdgas hergestellt. Würde Wasserstoff aus elektrischer Energie über die Elektrolyse hergestellt (was energetisch nur Sinn macht, falls dazu regenerativer Strom verwendet wird, der aus zeitlichen oder örtlichen Gründen im Überfluss vorhanden ist), könnten Brennstoffzellenfahrzeuge auch als Elektrofahrzeuge gelten, weil sie dann ähnliche Konsequenzen hätten wie batterieelektrische Fahrzeuge. In der vorliegenden Studie werden Brennstoffzellenfahrzeuge jedoch nicht untersucht. 
Hybridfahrzeuge: Hybridfahrzeuge (hybrid electric vehicle, HEV) werden teilweise (paralleler Hybrid sowie Toyota-Hybrid-System) oder ganz (serieller Hybrid) von einem Elektromotor angetrieben. Zu unterscheiden ist zwischen klassischem Hybrid (HEV) sowie Plug-In-Hybriden (plug-in hybrid electric vehicle, PHEV), welche die Batterie auch direkt ab Stromnetz aufladen können. Hybridfahrzeuge, welche ausschliesslich Benzin oder Diesel tanken, aber nicht an der Steckdose aufgeladen werden können, wie Toyota Prius, Honda Civic hybrid usw., zählen a priori nicht zu den Elektrofahrzeugen im Sinne der vorliegenden Definition. Plug-In-Hybride können, müssen aber nicht als Elektromobile aufgefasst werden: Wenn die Batteriekapazität eine nominale Reichweite von zirka 20 Kilometern aufweist, kann im Alltag (wenn das Fahrzeug vor allem im Agglomerationsverkehr eingesetzt wird) bereits mehr als die Hälfte der Fahrleistung mit Elektrizität aus stationären Quellen zurückgelegt werden. Dann handelt es sich nach unserer Definition um Elektromobilität (die Reichweite von 20 Kilometern ist nicht technisch begründet, sondern ergibt sich aus der erforderlichen Reichweite, um de facto im Alltag mindestens 50 Prozent der Fahrleistung mit der in der Batterie gespeicherten Energie zurücklegen zu können). Da die Grenze von 20 Kilometern eher niedrig ist und absehbar von nahezu allen PHEV erfüllt werden wird, und da ausserdem anzunehmen ist, dass eher jene Konsumenten PHEV erwerben, welche vom Einsatzprofil her einen grossen Teil aller Fahrzeugkilometer im Alltag elektrisch zurücklegen können, zählen wir PHEV pauschal zur Elektromobilität.

Range Extender: Wesentlich ist auch hier die Kapazität der Batterie. Wenn solche Fahrzeuge eine Batteriekapazität für ungefähr 20 Kilometer Reichweite aufweisen, handelt es sich um Elektromobile. Bei allen aktuell angekündigten Range Extender ist diese Bedingung erfüllt. Die Begrifflichkeiten zwischen PlugIn-Hybrid, Range Extender sowie Range Extension sind allerdings noch nicht geklärt, in der Literatur sind hierzu unterschiedliche Abgrenzungen zu finden. Für die vorliegende Studie wird Range Extender als Synonym für PHEV aufgefasst.

\subsection{Methodisches Vorgehen für die quantitative Berechnung der Chancen und Risiken}

Die Beurteilung der Elektromobilität hängt wesentlich davon ab, wie viel Elektromobilität es denn geben wird. Bei einem schnellen Aufbau der Elektrofahrzeugflotte sind Rohstoffprobleme und Anpassungsprobleme bei der Energiebereit- 
stellung und der Fahrzeugbetankung eher zu erwarten als bei einem langsameren Aufbau. Dazu entwickeln wir konsistente Szenarien. Sie variieren in der Geschwindigkeit des Aufbaus der Elektrofahrzeugflotte; davon wiederum ist abhängig, wie sich das Energiesystem sowie das übrige Verkehrssystem ausrichten müssten.

Die Szenarien postulieren, basierend auf den Annahmen für die Energiestrategie 2050 des Bundesrates, Top-Down-Effizienzziele für neu zugelassene Personenwagen, welche jedes Jahr steigen. Für eine konsistente Technikfolgenabschätzung zur Elektromobilität muss allerdings zuerst analysiert werden, wie sich dieser Top-Down-Verlauf aus Effizienzfortschritten von Einzelkomponenten bottom-up erklären und nachmodellieren lässt. Dazu muss auf Ebene der Komponenten und der Fahrzeuge die heutige Ausgangslage sowie die absehbare Entwicklung beschrieben werden.

Ein Kernmodul der Technikfolgenabschätzung zur Elektromobilität bildet die Lebenszyklusanalyse; die Lebensphasen der Herstellung, Nutzung und Entsorgung werden dabei zusammengefasst. Welcher Strommix hier zugrunde gelegt wird, hat grossen Einfluss auf die Resultate. In dieser Studie wird, wie für die Lebenszyklusanalyse allgemein üblich, die Durchschnittsbetrachtung verwendet (d.h., die Elektrofahrzeuge verwenden den durchschnittlichen in der Schweiz konsumierten Strommix). Die marginale Betrachtungsweise (würde ein Plus von Elektrofahrzeugen mit einem zusätzlichen Kohlekraftwerk betrieben oder aber umgekehrt mit vielen dezentralen Fotovoltaikanlagen auf Garagendächern?) wird in Sensitivitätsanalysen miteinbezogen.

Die Lebenszyklusanalyse vermag nicht alle relevanten Aspekte zu integrieren. Die Ressourcenverfügbarkeit in kurzer sowie mittlerer Frist, zusammen mit der allfälligen Konkurrenzierung der Elektromobilität durch andere Anwendungsfelder sowie die volkswirtschaftliche Beurteilung stellen zusätzliche wichtige Elemente der Technikfolgenabschätzung dar. Auch Reboundeffekte könnten auftreten und zu vermehrter Mobilität führen.

Die Resultate werden, je nach Szenario, zusammengeführt und einer Gesamtbeurteilung unterzogen. Unter Berücksichtigung der Grenzen der Genauigkeit wird ein Fazit gezogen. Darauf aufbauend formulieren wir Empfehlungen für Entscheidungsträger, die dabei helfen sollen, negative Folgen im weiteren Umgang mit der Elektromobilität zu antizipieren und möglichst zu vermeiden. 


\section{Definition der drei Szenarien}

\subsection{Systemgrenze und zu definierende Parameter}

Die vorliegende TA-SWISS-Studie verwendet den Szenarienansatz, wozu sowohl ein Trendszenario als auch normative Szenarien entwickelt werden. Sie bestehen aus Beschreibungen eines möglichen Stands des Mobilitäts- und Energiesystems der Schweiz in den Jahren 2035 und 2050. Die Szenarien gehen zwar von der heutigen Situation aus, setzen zum Teil aber auch grössere Innovationsschritte sozioökonomischer Art voraus. Die Stärke solcher normativer Szenarien besteht darin, vom Zukunftszeitpunkt aus zurück in die Gegenwart zu schauen («Backcasting») und auf diese Weise "von der Zukunft zu lernen» und Handlungspotenziale für die nahe Zukunft zu identifizieren. Die Szenarien sind im Kontext von Entwicklungen in den Bereichen Umwelt, Gesellschaft und Wirtschaft zu betrachten: Einerseits beeinflussen diese Entwicklungen den Bereich der Antriebe, Infrastrukturen, Fahrzeuge und Verkehrsnachfrage; andererseits wirkt der «innere Kreis» auf diese zurück. Grundlegende Arbeiten, welche für die Definition der Szenarien herangezogen wurden, sind IEA (2010), BFE (2012a, 2012b), BAFU (2012) sowie Bundesrat (2012). Die Eckdaten wurden dabei, wo immer möglich, in Übereinstimmung gebracht mit den Energieperspektiven des BFE, den Bevölkerungsprognosen des BFS sowie der Entwicklung des Personenverkehrs des ARE.

Elektromobilität umfasst die Fahrzeuge selber (Zweiräder und Kleinst-EV sowie «normale» Autos) sowie das Energiesystem (inklusive Ladeinfrastruktur) und «weiche» Faktoren wie die Geschäftsmodelle und Angebotskonzepte (inkl. ITLösungen für Reichweiten- und Ladestations-Planung und neue Car-SharingAnsätze). Auf allen Ebenen ist in Zukunft mit deutlichen Entwicklungen zu rechnen. Der Boom bei den elektrischen Zweirädern hat bereits vor einigen Jahren begonnen, es ist absehbar, dass bis etwa 2020 eine Vielzahl von Fahrrädern und kleinen Motorrädern einen elektrischen (Zusatz-)Antrieb aufweisen werden. Bei den Autos sind erst Ende 2010 die ersten wirklichen Serienfahrzeuge mit Elektroantrieb auf den Markt gekommen. Es wird bis mindestens 2017 dauern, bis weltweit eine Million batterieelektrische Autos hergestellt sind und durch die «Economy Of Scales» nennenswerte Kostensenkungseffekte in der Fertigung eintreten. Bis zirka 2020 werden sich auch die Leistungsdichten um etwa 50 Prozent erhöhen und die Kosten je batterieelektrische Kilowattstunde werden 
sich ungefähr halbieren. Mit weitergehenden Fortschritten wird in dieser Studie jedoch nicht gerechnet. Technologiesprünge sind zwar grundsätzlich möglich, können aber nicht im Rahmen einer Technikfolgenabschätzung antizipiert werden.

Die bei Elektromobilen zu erwartenden Entwicklungen sind den künftigen Fortschritten konventionell angetriebener Fahrzeuge gegenüberzustellen: In der EU27 wie auch in der Schweiz gilt für die PKW-Neuzulassungen des Jahres 2015 der Zielwert von $130 \mathrm{Gramm} \mathrm{CO}_{2}$-Emissionen pro Kilometer, als Fernziel (frühestens für das Jahr 2020, wahrscheinlich einige Jahre später) gelten 95 Gramm. Dies bedingt für die nächsten zehn Jahre Effizienzfortschritte von rund 4 Prozent pro Jahr. Um diese Werte zu erreichen, werden Elektrofahrzeuge ein Teil der Lösung sein, da sie keine $\mathrm{CO}_{2}$-Emissionen verursachen. Bis 2016 werden in der Schweiz die Elektromobile keine zahlenmässig nennenswerte Rolle spielen. Bis 2020 werden je nach Szenario 50000 bis maximal 100000 Elektrofahrzeuge in der Schweiz immatrikuliert sein (de Haan 2011).

Knappheit kann verursacht werden durch die steigende Nachfrage nach Elektromobilität, aber auch durch zunehmenden Bedarf in anderen Sektoren. In den Szenarien muss deshalb auch betrachtet werden, welche anderen Anwendungen seltene Materialressourcen benötigen.

\subsection{Eckpunkte der Szenarien}

Die Szenarien beruhen auf einem Business-as-Usual-Szenario, welches als Referenz für weitere zwei Szenarien dient. Diese sind jeweils von einer unterschiedlichen Marktdurchdringung der Elektrofahrzeuge gekennzeichnet, welche sich aus den zugrunde liegenden Annahmen ergeben und nicht ex ante festgelegt wurden.

Bei den Szenarien handelt es sich um denkbare und mögliche Zustände, welche in sich konsistent sein sollten. Es wird aber keinerlei Aussage darüber getroffen, mit welcher Wahrscheinlichkeit sie eintreten werden oder welches der drei Szenarien die höchste Wahrscheinlichkeit aufweist. In der Realität kann es auch Zwischenzustände geben, die aus einer Mischung der verschiedenen Szenarien bestehen. Da die Szenarien den wahrscheinlichen Entwicklungsraum (Spielmann et al. 2005) aufspannen, eignen sie sich für den Zweck einer Technikfolgenabschätzung. Es wurden aber bewusst keine Extremszenarien gewählt: Der künftige Marktanteil der Elektromobilität wird weder null noch 100 Prozent betragen. 


\begin{tabular}{|c|c|c|c|}
\hline Szenario & Business-as-Usual (BAU) & Effizienz (EFF) & Connected Mobility (COM) \\
\hline $\begin{array}{l}\text { Storyline } \\
\text { kurz }\end{array}$ & $\begin{array}{l}\text { Der «normale» technische } \\
\text { Fortschritt soll angewandt } \\
\text { werden, damit die Autos jährlich } \\
\text { effizienter, aber nicht } \\
\text { wesentlich kleiner werden }\end{array}$ & $\begin{array}{l}\text { Autos müssen einen grösseren } \\
\text { Beitrag zur Energieeinsparung } \\
\text { leisten als bisher. Dabei wird in } \\
\text { Kauf genommen, dass Autos im } \\
\text { Durchschnitt kleiner werden. }\end{array}$ & $\begin{array}{l}\text { Wie «Effizienz»; zusätzlich } \\
\text { kommt es zu Verhaltensände- } \\
\text { rungen hin zu einer stärker } \\
\text { «vernetzten Mobilität»: Längere } \\
\text { Fahrten werden meist mit ÖV } \\
\text { kombiniert }\end{array}$ \\
\hline $\begin{array}{l}\text { Storyline } \\
\text { länger }\end{array}$ & $\begin{array}{l}\text { Fortsetzung der bisherigen } \\
\text { Verkehrs- und Energiepolitik, } \\
\text { ohne neuartige politische } \\
\text { Massnahmen im Bereich. Das } \\
130 \mathrm{~g} \text {-Ziel für Neuwagen wird } \\
2015 \text { erreicht, das } 95 \mathrm{~g}-Z i e l \text { für } \\
\text { ein Stichjahr zwischen } 2020 \\
\text { und } 2025 \text { festgelegt. }\end{array}$ & $\begin{array}{l}\text { Verstärkte Förderung von } \\
\text { energieeffizienten Antrieben für } \\
\text { den MIV, jedoch ohne } \\
\text { technologie-spezifische } \\
\text { Förderung und staatliche } \\
\text { Vorinvestitionen in technologie- } \\
\text { spezifische Infrastruktur (wie } \\
\text { z.B. Ladeinfrastruktur). }\end{array}$ & $\begin{array}{l}\text { «Pull-» (Echtzeit-Info zu freien } \\
\text { P+R-Parkplätze/Ladestationen } \\
\text { +ÖV-Situation) und «Push-» } \\
\text { Faktoren (dynamisches Road } \\
\text { Pricing, vermehrte Staugefahr) } \\
\text { machen Kombination mit ÖV } \\
\text { attraktiver. Damit Wegfall der } \\
\text { Reichweiten-Problematik, und } \\
\text { Akzeptanz von Kleinstfahrzeu- } \\
\text { gen: Man setzt stark auf } \\
\text { Elektroautos }\end{array}$ \\
\hline Ausland & $\begin{array}{l}\text { Es wird angenommen, dass } \\
\text { andere Industriestaaten eine } \\
\text { ähnliche Politik verfolgen. Die } \\
\text { Schwellenländer verfolgen eine } \\
\text { Wachstumspolitik. }\end{array}$ & $\begin{array}{l}\text { Es wird angenommen, dass } \\
\text { andere Industriestaaten eine } \\
\text { ähnliche Politik verfolgen. Die } \\
\text { Schwellenländer verfolgen } \\
\text { zunächst eine Wachstums-, } \\
\text { später eine Effizienzpolitik. }\end{array}$ & $\begin{array}{l}\text { Es wird angenommen, dass } \\
\text { andere Industriestaaten eine } \\
\text { ähnliche Politik verfolgen. Die } \\
\text { Schwellenländer verfolgen } \\
\text { zunächst eine Wachstums-, } \\
\text { später eine Elektropolitik. }\end{array}$ \\
\hline Klimapolitik & $\begin{array}{l}\text { Schwach; Abweichung von } \\
2^{\circ} \text {-Ziel akzeptiert }\end{array}$ & $\begin{array}{l}\text { Stark. Gibt der Energiepolitik } \\
\text { Rückhalt. Fokus auf Reduktion } \\
\text { des Energieverbrauchs. }\end{array}$ & $\begin{array}{l}\text { Stark. Gibt der Energiepolitik } \\
\text { Rückhalt. Fokus auf schnellen } \\
\text { Ausbau erneuerbarer Energien }\end{array}$ \\
\hline $\begin{array}{l}\text { Energie- } \\
\text { politik }\end{array}$ & $\begin{array}{l}\text { Die neue Energiepolitik wird } \\
\text { langsam umgesetzt. AKW } \\
\text { bleiben lange am Netz, neue } \\
\text { Grosskraftwerke werden } \\
\text { gebaut, die CO2-Emissionen } \\
\text { in Kauf genommen, der Ausbau } \\
\text { der erneuerbaren Energien } \\
\text { erfolgt nur zögerlich und v.a. im } \\
\text { Ausland }\end{array}$ & $\begin{array}{l}\text { Stärkerer Fokus auf Energie- } \\
\text { effizienz und eine gewisse } \\
\text { Autonomie. CH hat eine } \\
\text { Vorreiterrolle bei Effizienzvor- } \\
\text { schriften für Gebäude, Geräte, } \\
\text { Prozesse, Personenwagen. } \\
\text { Sektorielles Vorgehen ohne } \\
\text { grossen Einbezug von } \\
\text { Systemeffekten. Förderung der } \\
\text { erneuerbaren Energien im } \\
\text { Inland. }\end{array}$ & Wie Effizienz \\
\hline Ressourcen & $\begin{array}{l}\text { Infolge globaler Wohlstands - } \\
\text { zunahme und Mobilitäts-zunah- } \\
\text { me Verdreifachung des } \\
\text { Fahrzeugbestandes auf 2,1 } \\
\text { Milliarden PKW/LNF 2050, } \\
\text { höhere Nachfrage nach } \\
\text { Ressourcen. }\end{array}$ & $\begin{array}{l}\text { Dank der höheren Effizienz und } \\
\text { abnehmenden mittlerern } \\
\text { Fahrzeuggrösse nimmt die } \\
\text { Nachfrage nach Schlüsselres- } \\
\text { sourcen leicht ab. }\end{array}$ & $\begin{array}{l}\text { Das Wachstum der Elektromo- } \\
\text { bilität führt zu einer verstärkten } \\
\text { Nachfrage nach den } \\
\text { entsprechenden Schlüsselres- } \\
\text { sourcen. }\end{array}$ \\
\hline Gesellschaft & $\begin{array}{l}\text { Keine Änderungen gegenüber } \\
\text { heute. }\end{array}$ & $\begin{array}{l}\text { Regressive Effekte infolge } \\
\text { höherer Mobilitätskosten }\end{array}$ & $\begin{array}{l}\text { Individuelle Mobilität } \\
\text { vergleichbar mit BAU. }\end{array}$ \\
\hline Datenschutz & $\begin{array}{l}\text { Keine Änderungen gegenüber } \\
\text { heute. }\end{array}$ & $\begin{array}{l}\text { Höhere Effizienz, frühere } \\
\text { Einführung von Car-To-Car- } \\
\text { und Infrastructure-To-Car- } \\
\text { Kommunikation. }\end{array}$ & $\begin{array}{l}\text { Höherer Anteil an Kleinstfahr- } \\
\text { zeugen, frühere Einführung von } \\
\text { Car-To-Car- und Infrastructure- } \\
\text { To-Car-Kommunikation. }\end{array}$ \\
\hline
\end{tabular}

\section{Tabelle 1: $\quad$ Definition der Szenarien: Storylines und Rahmenbedingungen.}


Tabelle 1 gibt die Hauptrichtungen und Eckpunkte der drei Szenarien wieder. Das erste Szenario ist dabei ein Trendszenario; es beschränkt sich nicht auf politische Massnahmen, wie sie heute schon beschlossen sind, sondern umfasst auch wahrscheinliche künftige politische Massnahmen, welche einer Fortsetzung der bisherigen Politik entsprechen. Die Bezeichnung dieses ersten Szenarios als «Business As Usual» wurde in Anlehnung an die bundesrätliche Energiestrategie gewählt, welche ihrerseits ein Weiter-wie-bisher-Szenario kennt, welches ein Trendszenario ist, dabei aber ebenfalls absehbare und wahrscheinliche neue politische Massnahmen einschliesst.

\subsection{Entwicklung von PKW-Politik, -Angebot und -Nachfrage}

Im Bereich der konventionellen Verbrennungsmotoren basiert die vorliegende Studie auf dem 130g-Szenario aus asa (2011). Damit wird explizit modelliert, dass auch die konventionell angetriebenen Fahrzeuge jährlich besser werden, und zwar in den Jahren bis 2020 mit hohen jährlichen Fortschrittsraten (Tabelle 2).

Die Tabelle 3 zeigt die Entwicklung der regulatorischen $\mathrm{g} \mathrm{CO}_{2} / \mathrm{km}$-Mittelwerte. Zusätzliche Informationen finden sich in Anhang A4 (Tabelle 29). Es handelt sich hier um die Normwerte gemäss Typprüfzyklus nach neuem europäischen Fahrzyklus NEFZ, die verwendeten $\mathrm{g} \mathrm{CO}_{2} / \mathrm{km}$-Werte für $\mathrm{PHEV}$ und $\mathrm{EV}$ sind die regulatorisch vorgegebenen Werte im Rahmen des Vollzugs zur $130 \mathrm{~g} \mathrm{CO}_{2} / \mathrm{km}-\mathrm{Re}-$ gulierung (bzw. für den $95 \mathrm{~g} \mathrm{CO}_{2} / \mathrm{km}$-Zielwert, für welchen wir ein Phasing-In ab 2020 annehmen). Für die Lebenszyklus-Berechnungen im nächsten Kapitel werden hingegen reale Energieverbrauchswerte sowie andere Strommixe unterlegt. 


\begin{tabular}{|c|c|c|c|}
\hline Szenario & BAU & Effizienz (EFF) & Connected Mobility (COM) \\
\hline Technik & $\begin{array}{l}\text { Autonomer technischer } \\
\text { Fortschritt, getrieben durch } \\
\text { das } 130 \mathrm{~g} \text {-Ziel für } 2015 \text { und } \\
\text { das } 95 \mathrm{~g} \text {-Ziel für ca. } 2020-25 \text {; } \\
\text { Fortschritt bei Verbrennungs- } \\
\text { motoren bis } 2025 \text { jährlich } \\
\text { grösser als bei Elektromobi- } \\
\text { len, danach gleich gross bis } \\
\text { ca. } 2035\end{array}$ & $\begin{array}{l}\text { Weil das staatliche Handeln } \\
\text { auf Effizienz, aber nicht } \\
\text { technologiespezifisch auf } \\
\text { Elektroantriebe zielt, treten } \\
\text { Huhn-Ei-Probleme auf. Ein } \\
\text { wesentlicher Teil der PKW } \\
\text { wird weiterhin mit (teilweise } \\
\text { hybridisierten) Verbrennungs- } \\
\text { motoren betrieben }\end{array}$ & $\begin{array}{l}\text { Schnellere technische Fort- } \\
\text { schritte und schnelleres } \\
\text { Einsetzen der Skaleneffekte. } \\
\text { Durchbrechung von } \\
\text { Huhn-Ei-Problemen infolge } \\
\text { der vermehrten Intermodalität } \\
\text { (ÖV für lange Teilstrecken; } \\
\text { damit keine Reichweiten- } \\
\text { problematik) }\end{array}$ \\
\hline Antriebe & $\begin{array}{l}\text { Die politischen Effizienzvor- } \\
\text { ga-ben richten sich am } \\
\text { Fortschritt bei den Verbren- } \\
\text { nungsmotoren aus, welche } \\
\text { den wichtigsten Teil der } \\
\text { Antriebe ausmachen. } \\
\text { Elektroantriebe setzen sich in } \\
\text { Teilsegmenten (City Cars, } \\
\text { Flottenfahrzeuge) durch. }\end{array}$ & $\begin{array}{l}\text { Starke Rolle für Hybride (in } \\
\text { Industrieländern ca. } 50 \\
\text { Prozent im Jahr 2050) und } \\
\text { Plug-In-Hybride/reine EV. }\end{array}$ & $\begin{array}{l}\text { Elektroförderung schliesst } \\
\text { Plug-In-Hybride ein. } \\
\text { Verbrennungsmotoren bei } \\
\text { PKW noch als Range } \\
\text { Extender im Einsatz. }\end{array}$ \\
\hline $\begin{array}{l}\text { Energie- } \\
\text { preise }\end{array}$ & $\begin{array}{l}\text { Mittel bis hoch, aber nicht } \\
\text { sehr hoch (Öl: unter } 200 \\
\text { USD/bbl), d.h. «normale» } \\
\text { technische Fortschritte } \\
\text { führen im MIV-Bereich } \\
\text { bereits zu einer Abnahme der } \\
\text { Energiekosten je Kilometer. }\end{array}$ & $\begin{array}{l}\text { Hoch, übt starken Druck in } \\
\text { Richtung Effizienzstrategie } \\
\text { aus }\end{array}$ & Wie Effizienz \\
\hline Preise & $\begin{array}{l}\text { Pro Kilometer real günstiger } \\
\text { als heute }\end{array}$ & $\begin{array}{l}\text { Höhere Kaufkosten, über } \\
\text { Betriebsdauer amortisiert; } \\
\text { Effizienzstrategie führt aber } \\
\text { zu im Mittel kleineren } \\
\text { Fahrzeugen und damit zu } \\
\text { niedrigeren Kosten je } \\
\text { Kilometer. }\end{array}$ & $\begin{array}{l}\text { Wie Effizienz; zunehmende } \\
\text { Kombipakete aus ÖV-Zugang } \\
\text { und Kleinstfahrzeugen für die } \\
\text { erste/letzte Meile. }\end{array}$ \\
\hline $\begin{array}{l}\text { Zweiräder } \\
\text { und Kleinst- } \\
\text { fahrzeuge }\end{array}$ & $\begin{array}{l}\text { Elektrifizierung der Zweiräder } \\
\text { in Industrieländern. } \\
\text { Schattendasein von } \\
\text { Kleinst-Fahrzeugkonzepten, } \\
\text { weil das Durchschnittsauto } \\
\text { nicht kleiner wird. }\end{array}$ & $\begin{array}{l}\text { Zunahme Kleinst-Fahrzeuge. } \\
\text { Durchschnittsauto wird } \\
\text { kleiner und differenziert sich } \\
\text { in Langstreckenfahrzeuge } \\
\text { und lokal eingesetzte } \\
\text { Fahrzeuge. }\end{array}$ & $\begin{array}{l}\text { Wachstum von Kleinst- } \\
\text { Fahrzeugen. }\end{array}$ \\
\hline Sicherheit & $\begin{array}{l}\text { Passive Sicherheit bleibt } \\
\text { Hauptpfeiler. Akustische War- } \\
\text { nung des LV durch PHEV/ } \\
\text { HEV/EV wird normiert. }\end{array}$ & $\begin{array}{l}\text { Ab } 2030 \text { wird aktive } \\
\text { Sicherheit ein Handlungsfeld } \\
\text { der Politik. }\end{array}$ & $\begin{array}{l}\text { Ab } 2030 \text { wird aktive } \\
\text { Sicherheit ein Handlungsfeld } \\
\text { der Politik. }\end{array}$ \\
\hline
\end{tabular}

\section{Tabelle 2: $\quad$ Definition der Szenarien: Entwicklung des Angebots an Fahr- zeugen.}




\begin{tabular}{|c|c|c|c|c|c|c|c|c|c|}
\hline BAU & 2015 & 2016 & 2017 & 2018 & 2019 & 2020 & 2025 & 2030 & 2035 \\
\hline $\mathrm{g} \mathrm{CO}_{2} / \mathrm{km}$ - ICE (inkl. HEV) & 129.6 & 126.2 & 122.9 & 119.6 & 116.5 & 113.4 & 105.8 & 98.7 & 92.0 \\
\hline $\mathrm{g} \mathrm{CU}_{2} / \mathrm{km}$ - HHEV & 50 & 40 & 40 & 40 & 40 & 40 & 30 & 25 & 20 \\
\hline $\mathrm{g} \mathrm{CO}_{2} / \mathrm{km}$ - EV & 0 & 0 & 0 & 0 & 0 & 0 & 0 & 0 & 0 \\
\hline $\mathrm{g} \mathrm{CO}_{2} / \mathrm{fzkm}$ - Mittelwert & 128.8 & 125.3 & 122.0 & 118.8 & 115.7 & 112.6 & 102.6 & 88.9 & 71.9 \\
\hline EFF & 2015 & 2016 & 2017 & 2018 & 2019 & 2020 & 2025 & 2030 & 2035 \\
\hline $\mathrm{g} \mathrm{CO}_{2} / \mathrm{km}$ - ICE (inkl. HEV) & 129.6 & 126.2 & 122.9 & 119.6 & 116.5 & 113.4 & 106.9 & 102.5 & 98.3 \\
\hline $\mathrm{g} \mathrm{CU}_{2} / \mathrm{km}$ - HHEV & 50 & 40 & 40 & 40 & 40 & 40 & 30 & 25 & 20 \\
\hline $\mathrm{g} \mathrm{CO}_{2} / \mathrm{km}-\mathrm{EV}$ & 0 & 0 & 0 & 0 & 0 & 0 & 0 & 0 & 0 \\
\hline $\mathrm{g} \mathrm{CO}_{2} / \mathrm{fzkm}$ - Mittelwert & 128.8 & 125.2 & 121.7 & 118.2 & 114.7 & 111.3 & 97.5 & 77.4 & 50.5 \\
\hline com & 2015 & 2016 & 2017 & 2018 & 2019 & 2020 & 2025 & 2030 & 2035 \\
\hline $\mathrm{g} \mathrm{CO}_{2} / \mathrm{km}$ - ICE (inkI. HEV) & 129.6 & 123.9 & 118.5 & 113.3 & 108.3 & 103.6 & 94.4 & 90.6 & 86.8 \\
\hline $\mathrm{g} \mathrm{CU}_{2} / \mathrm{km}$ - HHEV & 50 & 40 & 40 & 40 & 40 & 40 & 30 & 25 & 20 \\
\hline $\mathrm{g} \mathrm{CO}_{2} / \mathrm{km}-\mathrm{EV}$ & 0 & 0 & 0 & 0 & 0 & 0 & 0 & 0 & 0 \\
\hline $\mathrm{g} \mathrm{CO}_{2} / \mathrm{fzkm}$ - Mittelwert & 128.8 & 122.8 & 117.0 & 111.6 & 106.3 & 101.1 & 82.4 & 55.4 & 32.8 \\
\hline
\end{tabular}

Tabelle 3: $\quad \quad \quad g \mathrm{CO}_{2} / \mathrm{km}$-Mittelwerte der PKW-Neuzulassungen, getrennt für ICE/HEV, PHEV und EV sowie für die Gesamtheit aller Neuzulassungen.

Die nachstehende Tabelle zeigt die angenommenen Fahrleistungen sowie den jeweils daraus resultierenden Energieverbrauch. Zusätzliche Informationen finden sich in Anhang A4 (Tabelle 30 bis Tabelle 32).

\begin{tabular}{|c|c|c|c|c|c|c|c|c|c|}
\hline BAU & 2015 & 2016 & 2017 & 2018 & 2019 & 2020 & 2025 & 2030 & 2035 \\
\hline Fahrleistung der PKW [Mio. fzkm/a] & $63^{\prime} 414$ & $63^{\prime} 955$ & $64 \cdot 495$ & $65^{\prime} 036$ & $65^{\prime} 577$ & $66^{\prime} 117$ & $67 \cdot 749$ & $69^{\prime} 360$ & $71^{\prime} 363$ \\
\hline davon ICE BAU & $63^{\prime} 316$ & $63^{\prime} 818$ & $64^{\prime} 319$ & $64^{\prime} 819$ & $65^{\prime} 319$ & $65^{\prime} 818$ & $66^{\prime} 866$ & $66^{\prime} 501$ & $63^{\prime} 812$ \\
\hline davon PHEV: BAU & 47 & 66 & 86 & 106 & 127 & 147 & 439 & $1^{\prime} 427$ & $3^{\prime} 773$ \\
\hline davon EV: BAU & 47 & 66 & 86 & 106 & 127 & 147 & 439 & $1^{\prime} 427$ & $3^{\prime} 773$ \\
\hline \multicolumn{10}{|l|}{ Energie [PJ] } \\
\hline B/D/G, BAU & 150 & 147 & 144 & 140 & 137 & 134 & 119 & 107 & 95 \\
\hline E BAU & 0.07 & 0.10 & 0.13 & 0.16 & 0.18 & 0.2 & 0.6 & 1.9 & 4.9 \\
\hline \multicolumn{10}{|l|}{$\mathrm{CO}_{2}$-Emissionen [Mio t/a] } \\
\hline $\mathrm{B} / \mathrm{D} / \mathrm{G}, \mathrm{BAU}$ & 11.1 & 10.8 & 10.6 & 10.4 & 10.1 & 9.9 & 8.8 & 7.9 & 7.0 \\
\hline EFF & 2015 & 2016 & 2017 & 2018 & 2019 & 2020 & 2025 & 2030 & 2035 \\
\hline Fahrleistung der PKW [Mio. fzkm/a] & $63^{\prime} 414$ & $63^{\prime} 955$ & $64^{\prime} 495$ & $65^{\prime} 036$ & $65^{\prime} 577$ & $66^{\prime} 117$ & $67 \cdot 749$ & $69^{\prime} 360$ & $71^{\prime} 363$ \\
\hline davon ICE EF & $63^{\prime} 318$ & $63^{\prime} 812$ & $64^{\prime} 297$ & $64^{\prime} 768$ & $65^{\prime} 218$ & $65^{\prime} 649$ & $65^{\prime} 871$ & $62^{\prime} 857$ & $54^{\prime} 713$ \\
\hline davon PHEV: ET & 41 & 63 & 90 & 122 & 163 & 211 & 761 & $2^{\prime} 274$ & 4'924 \\
\hline davon EV: EF & 51 & 75 & 104 & 141 & 191 & 253 & 1'112 & $4^{\prime} 224$ & $11^{\prime} 721$ \\
\hline \multicolumn{10}{|l|}{ Energie [P] } \\
\hline B/D/G, 由下 & 150 & 147 & 144 & 140 & 137 & 133 & 118 & 103 & 84 \\
\hline E ET & 0.06 & 0.09 & 0.13 & 0.17 & 0.22 & 0.3 & 1.2 & 4.0 & 9.7 \\
\hline \multicolumn{10}{|l|}{$\mathrm{CO}_{2}$-Emissionen [Mio t/a] } \\
\hline B/D/G, 由于 & 11.1 & 10.8 & 10.6 & 10.3 & 10.1 & 9.8 & 8.7 & 7.6 & 6.2 \\
\hline com & 2015 & 2016 & 2017 & 2018 & 2019 & 2020 & 2025 & 2030 & 2035 \\
\hline \multirow{4}{*}{$\begin{array}{l}\text { Fahrleistung der PKW [Mio. fzkm/a] } \\
\text { davon ICE COM } \\
\text { davon PHEV: COM } \\
\text { davon EV: COM }\end{array}$} & $63^{\prime} 414$ & $63^{\prime} 955$ & $64^{\prime} 495$ & $65^{\prime} 036$ & $65^{\prime} 577$ & $66^{\prime} 117$ & $67 \cdot 749$ & $69^{\prime} 360$ & $71^{\prime} 363$ \\
\hline & $63^{\prime} 317$ & $63^{\prime} 804$ & $64^{\prime} 273$ & $64^{\prime} 725$ & $65^{\prime} 160$ & $65^{\prime} 563$ & $64^{\prime} 993$ & $59^{\prime} 466$ & $48^{\prime} 699$ \\
\hline & 41 & 67 & 99 & 136 & 179 & 230 & 852 & 1'962 & $2^{\prime} 228$ \\
\hline & 51 & 80 & 119 & 169 & 233 & 319 & 1'899 & $7^{\prime} 928$ & $20^{\prime} 431$ \\
\hline \multicolumn{10}{|l|}{ Energie [PJ] } \\
\hline B/D/G, COM & 150 & 147 & 143 & 139 & 135 & 131 & 110 & 88 & 65 \\
\hline E COM & 0.06 & 0.09 & 0.14 & 0.19 & 0.25 & 0.3 & 1.7 & 5.9 & 12.9 \\
\hline \multicolumn{10}{|l|}{$\mathrm{CO}_{2}$-Emissionen [Mio t/a] } \\
\hline B/D/G, COM & 11.1 & 10.8 & 10.6 & 10.3 & 10.0 & 9.6 & 8.1 & 6.5 & 4.8 \\
\hline
\end{tabular}

Tabelle 4: $\quad \mathrm{CO}_{2}$-Emissionen und Fahrleistungen, resultierender Energieverbrauch und Treibstoffabsätze ( $B=$ Benzin, $D=$ Diesel, $G=G a s$, $E=$ Elektrizität). 


\subsection{Marktanteile der Elektromobile bis 2035 und 2050}

Für die Entwicklung des Bestands der Elektromobile wird ein dreifacher Modellansatz verwendet:

- Kurzfristig: Eine obere Grenze für die Jahre bis ca. 2025 wird vorgegeben durch die maximale Produktionskapazität (siehe Kapitel 2.5);

- Mittelfristig: Für jedes Jahr werden in jedem Szenario EV-Angebotsflotten erstellt. Für jedes Jahr in jedem Szenario wird dann der schweizerische Neuwagenmarkt mit einer Mikrosimulation nachgebildet (siehe Kapitel 2.6);

- Langfristig: Für die Periode 2035 bis 2050 wird die Marktdurchdringung hin zu einem Endwert mit einer «S-Kurve» modelliert (siehe Kapitel 2.7).

Die Haupteigenschaften der Szenarien mit Bezug auf die technischen Eigenschaften von EV und ihre Beladung sind in untenstehender Tabelle spezifiziert.

\begin{tabular}{|l|l|l|l|}
\hline Szenario & BAU & Effizienz & Connected Mobility \\
\hline Steuern & $\begin{array}{l}\text { Wir gehen davon aus, dass auch mit Strom betriebene Fahrzeuge mittelfristig besteuert } \\
\text { werden (Übergang zu einer fahrleistungsabhängigen Besteuerung, welche nicht von der } \\
\text { Treibstoffart abhängt). Dieser Übergang sollte stattfinden, bevor der Kaufpreis von Elektro- } \\
\text { fahrzeugen soweit sinkt, dass deren Marktanteile relevant werden und eine ausbleibende } \\
\text { Besteuerung zu Reboundeffekten führen würde (je nach Szenario früher oder später im } \\
\text { Zeitraum 2020-2030). }\end{array}$ \\
\hline $\begin{array}{l}\text { Lade- } \\
\text { stationen }\end{array}$ & $\begin{array}{l}\text { Ladeinfrastruktur entsteht } \\
\text { ohne staatliche Beeinflus- } \\
\text { sung; Normierung der } \\
\text { Stecker usw. }\end{array}$ & Wie BAU & $\begin{array}{l}\text { Der Staat greift vor allem koordinierend ein, } \\
\text { weil die Elektroflotte schnell ausgebaut werden } \\
\text { soll: Entwicklung (mit anderen Staaten) eines } \\
\text { Standard-Schnellladesteckers. Planung der } \\
\text { Schnelllade-stationen entlang Autobahnen. }\end{array}$ \\
\hline
\end{tabular}

Tabelle 5: $\quad$ Definition der Szenarien: Elektromobile.

\section{Zurzeit produzierte oder angekündigte Elektrofahrzeuge}

Nachstehende Tabelle zeigt alle Elektromobile, welche aktuell auf dem Markt erhältlich sind oder dessen Markteintritt angekündigt wurde. Bei dieser Zusammenstellung wurde versucht, die wichtigsten technischen Parameter der Elektromobile in konsistenter Weise zusammenzutragen. Wo nötig, wurden fehlende Werte von 
Schwestermodellen (mit Benzinantrieb) übernommen oder geschätzt. Die Eckwerte dieser Elektromobile definieren den Startpunkt für unsere Annahmen zu den verfügbaren Elektromobilen in den nächsten Jahren (siehe Kapitel 2.6).

\begin{tabular}{|c|c|c|c|c|c|c|c|c|c|c|c|}
\hline car make & size class & PHEV & launch & $\begin{array}{r}\text { range } \\
{[\mathrm{km}]}\end{array}$ & $\begin{array}{r}\text { charge } \\
{[\mathrm{h}]}\end{array}$ & $\begin{array}{l}\text { pruce } \\
\text { [CHF] }\end{array}$ & $\begin{array}{r}\text { luggage } \\
\text { [liter] }\end{array}$ & $\begin{array}{r}\text { length } \\
{[\mathrm{mm}]}\end{array}$ & $\begin{array}{r}\text { mass } \\
{[\mathrm{kg}]}\end{array}$ & $\begin{array}{r}\text { battery } 1 \\
\text { [kWh] }\end{array}$ & $\begin{array}{r}\mathrm{km} / \mathrm{h} \\
{[\mathrm{s}]} \\
\end{array}$ \\
\hline Tesla Roadster & Micro & & 2010 & 343 & 3.5 & 135000 & 100 & 3937 & 1235 & 56 & 3.9 \\
\hline Mitsubishi i-MiEV & Micro & & 2010 & 150 & 8 & 45990 & 170 & 3480 & 1185 & 16 & 13.5 \\
\hline Chevrolet Volt & Compact & Yes & 2011 & 80 & 4 & 50490 & 310 & 4498 & 1715 & 16 & 9 \\
\hline Fisker Karma & Fullsize & Yes & 2011 & 80 & 9 & 129900 & 200 & 4996 & 2200 & 20 & 5.9 \\
\hline $\begin{array}{l}\text { Citroen zEro } \\
\text { Nissan leaf }\end{array}$ & $\begin{array}{l}\text { Micro } \\
\text { Compact }\end{array}$ & & $\begin{array}{l}2011 \\
2011\end{array}$ & $\begin{array}{l}150 \\
175\end{array}$ & $\begin{array}{l}8 \\
8\end{array}$ & $\begin{array}{l}45990 \\
49950\end{array}$ & $\begin{array}{l}170 \\
330\end{array}$ & $\begin{array}{l}3480 \\
4445\end{array}$ & $\begin{array}{l}1185 \\
1595\end{array}$ & $\begin{array}{l}16 \\
24\end{array}$ & $\begin{array}{l}13.5 \\
11.9\end{array}$ \\
\hline Toyota Prius Plug-in & Compact & Yes & 2012 & 23 & 1.5 & 45300 & 445 & 4460 & 1500 & 4.4 & 10.7 \\
\hline $\begin{array}{l}\text { Honda PEIV Platform } \\
\text { Volvo V60 Plug-In hybrid }\end{array}$ & $\begin{array}{l}\text { Compact } \\
\text { Compact }\end{array}$ & $\begin{array}{l}\text { Yes } \\
\text { Yes }\end{array}$ & $\begin{array}{l}2012 \\
2012\end{array}$ & $\begin{array}{l}24 \\
50\end{array}$ & $\begin{array}{l}1.5 \\
4.5\end{array}$ & $\begin{array}{r}48050 \\
50000\end{array}$ & $\begin{array}{l}310 \\
320\end{array}$ & $\begin{array}{l}4560 \\
4628\end{array}$ & $\begin{array}{l}1500 \\
2026\end{array}$ & $\begin{array}{r}6 \\
12\end{array}$ & $\begin{array}{r}10 \\
6.9\end{array}$ \\
\hline Opel Ampera & Compact & Yes & 2012 & 80 & 4 & 52900 & 310 & 4498 & 1712 & 16 & 9 \\
\hline $\begin{array}{l}\text { smart fortwo electric drive } \\
\text { Peugeot iOn }\end{array}$ & $\begin{array}{l}\text { Micro } \\
\text { Micro }\end{array}$ & & $\begin{array}{l}2012 \\
2012\end{array}$ & $\begin{array}{l}145 \\
150\end{array}$ & $\begin{array}{l}8 \\
8\end{array}$ & $\begin{array}{l}28290 \\
73800\end{array}$ & $\begin{array}{l}220 \\
170\end{array}$ & $\begin{array}{l}2695 \\
3480\end{array}$ & $\begin{array}{r}900 \\
1185\end{array}$ & $\begin{array}{r}17.6 \\
16\end{array}$ & $\begin{array}{l}12.9 \\
13.5\end{array}$ \\
\hline Renault Zoe & Compact & & 2012 & 160 & 8 & 34932 & 292 & 4086 & 1392 & 22 & 8 \\
\hline $\begin{array}{l}\text { Honda Jazz EV } \\
\text { Ford Focus Eectic }\end{array}$ & $\begin{array}{l}\text { Compact } \\
\text { Compact }\end{array}$ & & $\begin{array}{l}2012 \\
2012\end{array}$ & $\begin{array}{l}160 \\
160\end{array}$ & $\begin{array}{r}5.75 \\
3.5\end{array}$ & $\begin{array}{l}38500 \\
49000\end{array}$ & $\begin{array}{l}300 \\
277\end{array}$ & $\begin{array}{l}3900 \\
4361\end{array}$ & $\begin{array}{l}1460 \\
1614\end{array}$ & $\begin{array}{r}26.5 \\
23\end{array}$ & $\begin{array}{r}12.1 \\
10\end{array}$ \\
\hline Renault Fuence ZE & Compact & & 2012 & 160 & 8 & $47100^{*}$ & 317 & 4748 & 1543 & 22 & 9.9 \\
\hline $\begin{array}{l}\text { Renault Kangoo ZE } \\
\text { Renault Kangoo Maxi ZE }\end{array}$ & $\begin{array}{l}\text { Compact } \\
\text { Compact }\end{array}$ & & $\begin{array}{l}2012 \\
2012\end{array}$ & $\begin{array}{l}160 \\
160\end{array}$ & $\begin{array}{l}8 \\
8\end{array}$ & $\begin{array}{l}28404^{\star} \\
31500^{*}\end{array}$ & $\begin{array}{l}3000 \\
4000\end{array}$ & $\begin{array}{l}4213 \\
4597\end{array}$ & $\begin{array}{l}1410 \\
1483\end{array}$ & $\begin{array}{l}22 \\
22\end{array}$ & $\begin{array}{l}5.1 \\
5.1\end{array}$ \\
\hline Renault Twizy & 3-Wheeler & & 2012 & 115 & 4 & $9600^{*}$ & 40 & 2337 & 450 & \multicolumn{2}{|c|}{$7(<80 \mathrm{~km} / \mathrm{h})$} \\
\hline $\begin{array}{l}\text { Toyota RAV4 EV (US only) } \\
\text { Coda }\end{array}$ & $\begin{array}{l}\text { Fullsize } \\
\text { Compact }\end{array}$ & & $\begin{array}{l}2012 \\
2012\end{array}$ & $\begin{array}{l}140 \\
175\end{array}$ & $\begin{array}{r}18 \\
6\end{array}$ & $\begin{array}{r}80000^{*} \\
39900\end{array}$ & $\begin{array}{r}1031 \\
399\end{array}$ & $\begin{array}{l}4232 \\
4470\end{array}$ & $\begin{array}{l}1560 \\
1665\end{array}$ & $\begin{array}{l}90 \\
31\end{array}$ & $\begin{array}{r}8 \\
4.9\end{array}$ \\
\hline Toyota iQ EV & Micro & & 2012 & 91 & & 22950 & 80 & 2990 & 1120 & 24 & 5 \\
\hline $\begin{array}{l}\text { Tesla Model S } \\
\text { Tesla Model S }\end{array}$ & $\begin{array}{l}\text { Fullsize } \\
\text { Fullsize }\end{array}$ & & $\begin{array}{l}2013 \\
2013\end{array}$ & $\begin{array}{l}260 \\
370\end{array}$ & $\begin{array}{l}18 \\
30\end{array}$ & $\begin{array}{l}71250 \\
83750\end{array}$ & $\begin{array}{l}1042 \\
1042\end{array}$ & $\begin{array}{l}4978 \\
4978\end{array}$ & $\begin{array}{l}1735 \\
2071\end{array}$ & $\begin{array}{l}42 \\
70\end{array}$ & $\begin{array}{l}5.6 \\
5.6\end{array}$ \\
\hline Tesla Model S & Fullsize & & 2013 & 480 & 40 & 95250 & 1042 & 4978 & 2311 & 90 & 5.6 \\
\hline $\begin{array}{l}\text { Audi A1 e tron } \\
\text { Ford C-Max Energi }\end{array}$ & $\begin{array}{l}\text { Compact } \\
\text { Compact }\end{array}$ & $\begin{array}{l}\text { Yes } \\
\text { Yes }\end{array}$ & $\begin{array}{l}2013 \\
2013\end{array}$ & $\begin{array}{l}50 \\
50\end{array}$ & $\begin{array}{l}4 \\
3\end{array}$ & $\begin{array}{l}32150 \\
46863\end{array}$ & $\begin{array}{l}270 \\
765\end{array}$ & $\begin{array}{l}3954 \\
4409\end{array}$ & $\begin{array}{l}1200 \\
1510\end{array}$ & $\begin{array}{l}12 \\
10\end{array}$ & $\begin{array}{r}10.2 \\
10\end{array}$ \\
\hline WW E-UP! & Micro & & 2013 & 130 & 5 & 23186 & 80 & 3190 & 1100 & 18 & 11.3 \\
\hline Hyundai BlueOn & Micro & & 2013 & 140 & 6 & 26330 & 225 & 3585 & 1263 & 16.4 & 13.1 \\
\hline WW Golf blue e-motion & Compact & & 2013 & 150 & 8 & 36900 & 237 & 4199 & 1545 & 26.5 & 11.8 \\
\hline BMW i3 & Compact & & 2013 & 160 & 6 & 49200 & 200 & 3845 & 1250 & 27.6 & 7.9 \\
\hline $\begin{array}{l}\text { Mercedes BE-Cell } \\
\text { Chevrolet Volt MPV5 }\end{array}$ & $\begin{array}{l}\text { Compact } \\
\text { Compact }\end{array}$ & $\begin{array}{l}\text { Yes } \\
\text { Yes }\end{array}$ & $\begin{array}{l}2014 \\
2013\end{array}$ & $\begin{array}{r}100 \\
44.8\end{array}$ & $\begin{array}{l}5 \\
4\end{array}$ & $\begin{array}{l}53000 \\
50490\end{array}$ & $\begin{array}{l}486 \\
310\end{array}$ & $\begin{array}{l}4359 \\
4498\end{array}$ & $\begin{array}{l}1534 \\
1715\end{array}$ & $\begin{array}{l}11 \\
16\end{array}$ & $\begin{array}{r}11.6 \\
9\end{array}$ \\
\hline Tesla Model X & Fullsize & & 2014 & 370 & 30 & 84000 & 1042 & 4978 & 2071 & 60 & 4.4 \\
\hline Tesla Model X & Fullsize & & 2014 & 480 & 40 & 95250 & 1042 & 4978 & 2311 & 85 & 4.4 \\
\hline Audi A2 concept & Compact & & 2015 & 200 & 4 & 48050 & 310 & 3800 & 1150 & 31 & 9.3 \\
\hline
\end{tabular}

Tabelle 6: $\quad$ Liste der zurzeit angekündigten oder produzierten EV. Die Angaben zur Grösse des Gepäckraums, des Leergewichts und der benötigten Zeit für die Beschleunigung von 0 auf $100 \mathrm{~km} / \mathrm{h}$ wurden, wenn fehlend, geschätzt.

\section{Vorhersagen durch andere Forschergruppen}

Nachfolgende Tabelle zeigt im Überblick die Vorhersagen oder möglichen Verläufe (es handelt sich hier teilweise um Vorhersagen, teilweise um Szenarienberechnungen) des Anteils der Elektrofahrzeuge am Neuwagenmarkt und/oder am Gesamtautobestand von anderen Forschergruppen. Der Überblick differenziert nach der regionalen Bezugseinheit (Welt, EU, USA, Deutschland oder Schweiz). 


\begin{tabular}{|c|c|c|c|c|c|c|c|c|c|c|c|}
\hline \multirow[b]{2}{*}{ Quelle } & \multirow[b]{2}{*}{ Region } & \multicolumn{5}{|c|}{ Anteil in PKW-Neuzulassungen } & \multicolumn{5}{|c|}{ Anteil in PKW-Fahrzeugflotte } \\
\hline & & 2015 & 2020 & 2025 & 2030 & 2050 & 2015 & 2020 & 2025 & 2030 & 2050 \\
\hline Rosenfeld, Remes et al. (2011), p.78ff & World & & & & & & & $4 \%$ & & & \\
\hline Kalmbach, Bernhart et al. (2011), p.56 & World & & & $10 \%$ & & & & & & & \\
\hline J.D.Power (2010) & World & & $1.8 \%$ & & & & & & & & \\
\hline Hazimeh, Tweadey et al. (2010) & World & & $10 \%$ & & & & & & & & \\
\hline van Essen and Kampmann (2011) & World & & $4 \%$ & & & & & & & & \\
\hline Forbes (2012) & World & & $10 \%$ & & & & & & & & \\
\hline Rosenfeld, Remes et al. (2011), p.78ff & $\mathrm{EU}$ & & $16 \%$ & & & & & & & & \\
\hline McKinsey (2010), p.17: scenario 1 & EU & & & & & & & & & & $35 \%$ \\
\hline McKinsey (2010), p.17: scenario 2 & EU & & & & & & & & & & $70 \%$ \\
\hline van Essen and Kampmann (2011): scenario 1 & $\mathrm{EU}$ & $1 \%$ & $5 \%$ & $26 \%$ & $52 \%$ & & $0 \%$ & $1 \%$ & $6 \%$ & $18 \%$ & \\
\hline van Essen and Kampmann (2011): scenario 2 & $\mathrm{EU}$ & $0 \%$ & $3 \%$ & $11 \%$ & $19 \%$ & & $0 \%$ & $0 \%$ & $2 \%$ & $7 \%$ & \\
\hline van Essen and Kampmann (2011): scenario 3 & $\mathrm{EU}$ & $1 \%$ & $10 \%$ & $54 \%$ & $84 \%$ & & $0 \%$ & $1 \%$ & $12 \%$ & $33 \%$ & \\
\hline Becker et al. (2009): scenario baseline & US & $3 \%$ & $18 \%$ & $45 \%$ & $64 \%$ & & $1 \%$ & $4 \%$ & $11 \%$ & $24 \%$ & \\
\hline Becker et al. (2009): scenario high energy prices & US & $5 \%$ & $35 \%$ & $75 \%$ & $85 \%$ & & & & & & \\
\hline Becker et al. (2009): scenario operator subsidies & US & $10 \%$ & $49 \%$ & $81 \%$ & $86 \%$ & & & & & $46 \%$ & \\
\hline Alpiq (2009), p.23 & $\mathrm{CH}$ & & $50 \%$ & & & & & $15 \%$ & & & \\
\hline BFE (2010): scenario 1 & $\mathrm{CH}$ & & & & & & & $2.6 \%$ & & & \\
\hline BFE (2010): scenario 2 & $\mathrm{CH}$ & & & & & & & $6.6 \%$ & & & \\
\hline BFE (2010): scenario 3 & $\mathrm{CH}$ & & & & & & & $4.9 \%$ & & & \\
\hline BFE (2010): scenario 4 & $\mathrm{CH}$ & & & & & & & $2.2 \%$ & & & \\
\hline Zah, Binder et al. (2010): scenario conservative & $\mathrm{CH}$ & & & & $50 \%$ & & & & & & \\
\hline Zah, Binder et al. (2010): scenario optimistic & $\mathrm{CH}$ & & & $50 \%$ & & & & & & & \\
\hline asa (2011): scenario medium & $\mathrm{CH}$ & & $9 \%$ & & & & & $1 \%$ & & & \\
\hline asa (2011): scenario high & $\mathrm{CH}$ & & $17 \%$ & & & & & $2 \%$ & & & \\
\hline Shell (2009): scenario trend & $\mathrm{D}$ & & & & $2.5 \%$ & & & & & & \\
\hline Shell (2009): scenario alternative & D & & $3.3 \%$ & $6 \%$ & $10 \%$ & & & & & & \\
\hline
\end{tabular}

Tabelle 7: $\quad$ Vorhersagen zur Marktpenetration der Elektromobilität aus der wissenschaftlichen Literatur.

Nachstehende Abbildung zeigt die Bandbreite des Anteils an den PKW-Neuzulassungen für die regionalen Märkte EU, USA, Deutschland und Schweiz.

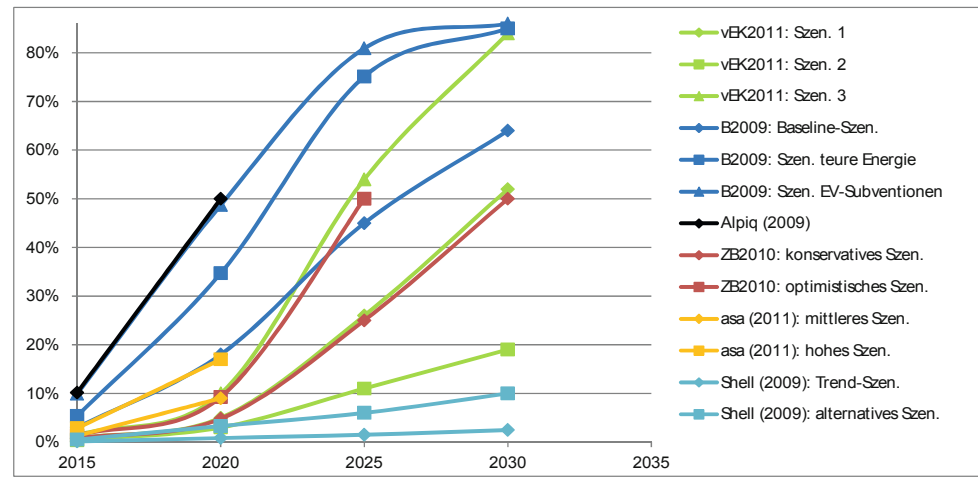

Abbildung 8: $\quad$ Mögliche Verläufe des Anteils der Elektrofahrzeuge am Neuwagenmarkt in der EU (vEK2011: van Essen und Kampman 2011), den USA (B2009: Becker et al. 2009), Deutschland (Shell 2009) und in der Schweiz (Alpiq 2009; ZB2010: Zah, Binder et al. 2010; asa 2011). 
Die in Abbildung 8 gezeigten Verläufe gehen weit auseinander; für Industrieländer werden generell höhere EV-Anteile genannt als im globalen Durchschnitt. Ursachen für die grossen Differenzen sind, dass einerseits PHEV teilweise einund teilweise ausgeschlossen sind. Anderseits kommen manche Verläufe eher normativen Zielszenarien gleich. Wir stellen sie deshalb einer Abschätzung der kurzfristigen Produktionskapazität gegenüber, um sie auf ihre Plausibilität überprüfen zu können.

\subsection{Produktionskapazität von Elektromobilen bis 2025}

Zur Abschätzung der Produktionskapazität gehen wir wie folgt vor: Ausgangspunkt ist die Weltproduktionskapazität von PKW gemäss OICA (2011), insgesamt 60,3 Millionen PKW im Jahre 2010. Wir nehmen für die vorliegende Abschätzung an, dass die relativen Marktanteile der Hersteller eingefroren bleiben, und lassen den Markt um jährlich 2,86 Prozent wachsen, damit sie im Jahre 2020 exakt 80 Millionen PKW jährlich erreicht (unsere Schätzung für den PKW-Absatz im Jahr 2020; der World Energy Outlook 2011 geht im New-Policy-Szenario sogar von 88 Millionen PKW im Jahr 2020 aus [IEA 2011, Fig. 3.11]). Tabelle 8 zeigt die gesamte Produktionskapazität in Fünf-Jahresschritten bis 2025 für die grössten Hersteller, wobei die 16 grössten festland-chinesischen Hersteller zusammengefasst wurden. Bei der Joint-Venture-Produktion, welche namentlich in China den grösseren Teil des Markts ausmacht, wurde die Herkunftsmarke verwendet. Beispiel: First Auto Works (FAW) hatten 2010 eine Totalproduktion von 2572 260, davon 896060 unter Eigenmarken (Markennamen FAW, Hongqi, Besturn, OuLai, Xiali, Haima, und KaiLi), zuzüglich 1676200 aus Joint-Venture-Produktion, welche in diesem Fall unter den Markennamen Volkswagen und Toyota erscheinen (siehe Tabelle 8).

Zur Abschätzung der Produktionskapazität für Elektrofahrzeuge wurde wie folgt vorgegangen:

- Die jährlich neu hinzukommende Produktionskapazität (Differenz der absoluten Produktion im Vergleich zum Vorjahr) wird addiert zu 8 Prozent der gesamten Vorjahresproduktionskapazität (Annahme: 8 Prozent aller Produktionslinien werden jährlich vollständig neu aufgebaut, d.h. im Mittel läuft eine Produktionslinie 12,5 Jahre). 
- Es wird angenommen, dass immer ein bestimmter Prozentsatz dieser jährlich neu konzipierten Produktionskapazität für die Produktion von EV verwendet werden könnte. Der Prozentsatz ist abhängig vom Kalenderjahr und von den Herstellergruppen (getrennt betrachtet werden Renault+Nissan, alle festlandchinesischen Hersteller, PSA+Mitsubishi, Toyota sowie alle übrigen Hersteller).

\begin{tabular}{|c|c|c|c|c|c|c|c|c|}
\hline & 2010 & 2011 & 2012 & 2013 & 2014 & 2015 & 2020 & 2025 \\
\hline TOYOTA & $7^{\prime} 268^{\prime} 000$ & $7^{\prime} 475^{\prime} 000$ & $7^{\prime} 689^{\prime} 000$ & $7^{\prime} 9099^{\prime} 000$ & $8^{\prime} 135^{\prime} 000$ & $8^{\prime} 368^{\prime} 000$ & $9^{\prime} 635^{\prime} 000$ & $11^{\prime} 093^{\prime} 000$ \\
\hline G.M. & $6^{\prime} 267^{\prime} 000$ & $6^{\prime} 446^{\prime} 000$ & $6^{\prime} 630^{\prime} 000$ & $6^{\prime} 820^{\prime} 000$ & $7^{\prime} 015^{\prime} 000$ & $7^{\prime} 216^{\prime} 000$ & $8^{\prime} 308^{\prime} 000$ & $9^{\prime} 566^{\prime} 000$ \\
\hline VOLKSWAGEN & $7^{\prime} 121^{\prime} 000$ & $7^{\prime} 324^{\prime} 000$ & $7^{\prime} 534^{\prime} 000$ & $7^{\prime} 7499^{\prime} 000$ & $7^{\prime} 971^{\prime} 000$ & $8^{\prime} 198^{\prime} 000$ & $9^{\prime} 440^{\prime} 000$ & $10^{\prime} 869^{\prime} 000$ \\
\hline China (16 OEM) & $5^{\prime} 725^{\prime} 000$ & $5^{\prime} 888^{\prime} 000$ & $6^{\prime} 057^{\prime} 000$ & $6^{\prime} 230$ '000 & $6^{\prime} 408^{\prime} 000$ & 6'591'000 & $7^{\prime} 589^{\prime} 000$ & $8^{\prime} 738^{\prime} 000$ \\
\hline HYUNDAI & $5^{\prime} 247^{\prime} 000$ & $5^{\prime} 397^{\prime} 000$ & $5^{\prime} 552^{\prime} 000$ & $5^{\prime} 710^{\prime} 000$ & $5^{\prime} 874^{\prime} 000$ & $6^{\prime} 0422^{\prime} 000$ & 6'956'000 & $8^{\prime} 009^{\prime} 000$ \\
\hline FORD & $2^{\prime} 959^{\prime} 000$ & $3^{\prime} 043^{\prime} 000$ & $3^{\prime} 130^{\prime} 000$ & $3^{\prime} 220^{\prime} 000$ & $3^{\prime} 312^{\prime} 000$ & $3^{\prime} 406^{\prime} 000$ & $3^{\prime} 9222^{\prime} 000$ & $4^{\prime} 516^{\prime} 000$ \\
\hline NISSAN & $3^{\prime} 142^{\prime} 000$ & $3^{\prime} 232^{\prime} 000$ & $3^{\prime} 324^{\prime} 000$ & $3^{\prime} 419^{\prime} 000$ & $3^{\prime} 517^{\prime} 000$ & $3^{\prime} 618^{\prime} 000$ & $4^{\prime} 165^{\prime} 000$ & $4^{\prime} 796^{\prime} 000$ \\
\hline HONDA & $3^{\prime} 592^{\prime} 000$ & $3^{\prime} 695^{\prime} 000$ & $3^{\prime} 800^{\prime} 000$ & $3^{\prime} 909^{\prime} 000$ & $4^{\prime} 021^{\prime} 000$ & $4^{\prime} 1366^{\prime} 000$ & $4^{\prime} 762^{\prime} 000$ & $5^{\prime} 483^{\prime} 000$ \\
\hline PSA & $3^{\prime} 215^{\prime} 000$ & $3^{\prime} 307^{\prime} 000$ & $3^{\prime} 401^{\prime} 000$ & $3^{\prime} 499 ' 000$ & $3^{\prime} 599^{\prime} 000$ & 3'701'000 & $4^{\prime} 262$ '000 & $4^{\prime} 907^{\prime} 000$ \\
\hline SUZUKI & $2^{\prime} 503^{\prime} 000$ & $2^{\prime} 575^{\prime} 000$ & $2^{\prime} 649^{\prime} 000$ & $2^{\prime} 724^{\prime} 000$ & $2^{\prime} 802^{\prime} 000$ & $2^{\prime} 882^{\prime} 000$ & $3^{\prime} 319^{\prime} 000$ & $3^{\prime} 821^{\prime} 000$ \\
\hline RENAULT & $2^{\prime} 396^{\prime} 000$ & $2^{\prime} 464^{\prime} 000$ & $2^{\prime} 535^{\prime} 000$ & $2^{\prime} 607^{\prime} 000$ & $2^{\prime} 682^{\prime} 000$ & $2^{\prime} 759^{\prime} 000$ & $3^{\prime} 176^{\prime} 000$ & $3^{\prime} 657^{\prime} 000$ \\
\hline FAT & 1'781'000 & 1'832'000 & $1^{\prime} 885^{\prime} 000$ & 1'939'000 & 1'994'000 & $2^{\prime} 051^{\prime} 000$ & $2^{\prime} 362^{\prime} 000$ & $2^{\prime} 719^{\prime} 000$ \\
\hline DAIMLR & 1'351'000 & $1 ' 390 ' 000$ & $1^{\prime} 430 ' 000$ & 1'471'000 & 1'513'000 & $1^{\prime} 556$ '000 & 1'792'000 & $2^{\prime} 063^{\prime} 000$ \\
\hline CHRYSLER & $340^{\prime} 000$ & $350^{\prime} 000$ & $360 ' 000$ & $370^{\prime} 000$ & $381^{\prime} 000$ & $392^{\prime} 000$ & $451^{\prime} 000$ & $519^{\prime} 000$ \\
\hline B.M.W. & $1^{\prime} 481^{\prime} 000$ & 1'524'000 & $1^{\prime} 567 ' 000$ & $1^{\prime} 612^{\prime} 000$ & $1^{\prime} 658 ' 000$ & 1'705'000 & 1'964'000 & $2^{\prime} 261^{\prime} 000$ \\
\hline MAZDA & 1'234'000 & $1^{\prime} 269 ' 000$ & 1'305'000 & 1'343'000 & 1'381'000 & $1^{\prime} 421^{\prime} 000$ & $1^{\prime} 636 ' 000$ & 1'883'00c \\
\hline MITSUBISHI & 1'057'000 & $1^{\prime} 087^{\prime} 000$ & 1'118'000 & 1'150'000 & 1'183'000 & 1'217'000 & $1^{\prime} 401^{\prime} 000$ & $1^{\prime} 613^{\prime} 000$ \\
\hline TATA & $579 ' 000$ & $596^{\prime} 000$ & $613^{\prime} 000$ & $630^{\prime} 000$ & $648^{\prime} 000$ & $667^{\prime} 000$ & $768^{\prime} 000$ & $884^{\prime} 000$ \\
\hline FUJ (Subaru) & $595^{\prime} 000$ & $612^{\prime} 000$ & $630^{\prime} 000$ & $648^{\prime} 000$ & $667^{\prime} 000$ & $686^{\prime} 000$ & $789^{\prime} 000$ & $909^{\prime} 000$ \\
\hline AVTOVAZ & $546^{\prime} 000$ & $561^{\prime} 000$ & $577^{\prime} 000$ & $594^{\prime} 000$ & $611^{\prime} 000$ & $628^{\prime} 000$ & $724^{\prime} 000$ & $833^{\prime} 000$ \\
\hline Mahindra & $167^{\prime} 000$ & $172^{\prime} 000$ & $176^{\prime} 000$ & $181^{\prime} 000$ & $187^{\prime} 000$ & $192^{\prime} 000$ & $221^{\prime} 000$ & $255^{\prime} 000$ \\
\hline Proton & $143^{\prime} 000$ & $147^{\prime} 000$ & $152^{\prime} 000$ & $156^{\prime} 000$ & $160^{\prime} 000$ & $165^{\prime} 000$ & $190^{\prime} 000$ & $219^{\prime} 000$ \\
\hline Kuozui & $115^{\prime} 000$ & $118^{\prime} 000$ & $122^{\prime} 000$ & $125^{\prime} 000$ & $129^{\prime} 000$ & $133^{\prime} 000$ & $153^{\prime} 000$ & $176^{\prime} 00 \mathrm{c}$ \\
\hline Porsche & $96^{\prime} 000$ & $98^{\prime} 000$ & $101^{\prime} 000$ & $104^{\prime} 000$ & $107^{\prime} 000$ & $110^{\prime} 000$ & $127^{\prime} 000$ & $146^{\prime} 000$ \\
\hline REST & 1'424'000 & $1^{\prime} 465^{\prime} 000$ & 1'507'000 & 1'550'000 & 1'594'000 & $1^{\prime} 640$ '000 & 1'888'000 & $2^{\prime} 174^{\prime} 000$ \\
\hline Summe & $60^{\prime} 344^{\prime} 000$ & $62^{\prime} 067^{\prime} 000$ & $63^{\prime} 844^{\prime} 000$ & $65^{\prime} 669^{\prime} 000$ & $67^{\prime} 549^{\prime} 000$ & $69^{\prime} 480^{\prime} 000$ & $80^{\prime} 000^{\prime} 000$ & $92^{\prime} 109^{\prime} 000$ \\
\hline
\end{tabular}

Tabelle 8: $\quad$ Produktion von PKW weltweit, für 2010 (gemäss OIAC 2011) und extrapoliert bis 2025 mit jährlicher Steigerungsrate von 2,86 Prozent (linear).

Das Ergebnis ist in Tabelle 9 zusammengefasst. Es zeigt, dass damit in etwa die folgenden externen Vorhersagen abgebildet werden können: Im Jahre 2011 wurden ca. 20000 EV produziert (Forbes 2012); die festlandchinesischen Hersteller gedenken im Jahre 2015 eine Million EV jährlich produzieren zu können (in unserem Modell, etwas zurückhaltender: 0,5 Millionen) (Guardian 2011), die Renault-Nissan-Gruppe will kumuliert bis 2015 1,5 Millionen EV hergestellt haben (Forbes 2012), im Jahre 2020 wird die EV-Produktionskapazität weltweit ca. 10 Prozent der Gesamtkapazität betragen (in unserem Modell: 11 Prozent). 


\begin{tabular}{|c|c|c|c|c|c|}
\hline & 2010 & 2015 & 2020 & 2025 & Summe 2010-15 \\
\hline TOYOTA & 0 & $160 ' 300$ & $814^{\prime} 300$ & $2^{\prime} 747^{\prime} 400$ & $257^{\prime} 900$ \\
\hline G.M. & 0 & $27^{\prime} 600$ & $140 ' 400$ & $473^{\prime} 800$ & $44^{\prime} 400$ \\
\hline VOLKSWAGEN & 0 & $31^{\prime} 400$ & $159^{\prime} 600$ & $538^{\prime} 400$ & $50^{\prime} 500$ \\
\hline China (16 OEM) & 0 & $505^{\prime} 000$ & $2^{\prime} 565^{\prime} 700$ & $8^{\prime} 6566^{\prime} 700$ & $812^{\prime} 600$ \\
\hline HYUNDAI & 0 & $23^{\prime} 100$ & $117^{\prime} 600$ & $3966^{\prime} 700$ & $37^{\prime} 200$ \\
\hline FORD & 0 & $13^{\prime} 100$ & $66^{\prime} 300$ & $223^{\prime} 700$ & $21^{\prime} 000$ \\
\hline NISSAN & 0 & $415^{\prime} 800$ & $2^{\prime} 112^{\prime} 300$ & $7^{\prime} 127^{\prime} 100$ & $669^{\prime} 000$ \\
\hline HONDA & 0 & $15^{\prime} 800$ & $80^{\prime} 500$ & $271^{\prime} 600$ & $25^{\prime} 400$ \\
\hline PSA & 0 & $70 ' 900$ & $360 ' 200$ & 1'215'300 & $114^{\prime} 100$ \\
\hline SUZUKI & 0 & $11^{\prime} 000$ & $56 ' 100$ & $189 ' 300$ & $17^{\prime} 700$ \\
\hline R日NAULT & 0 & $317^{\prime} 100$ & 1'610'700 & $5^{\prime} 434^{\prime} 400$ & $510^{\prime} 200$ \\
\hline FAT & 0 & 7'900 & $39^{\prime} 900$ & $134 ' 700$ & $12^{\prime} 700$ \\
\hline DAIMLRR & 0 & 6'000 & $30 ' 300$ & $102 ' 200$ & $9^{\prime} 600$ \\
\hline CHRYSLER & 0 & 1'500 & $7^{\prime} 600$ & $25^{\prime} 700$ & $2^{\prime} 400$ \\
\hline B.M.W. & 0 & $6^{\prime} 500$ & $33^{\prime} 200$ & $112^{\prime} 000$ & $10^{\prime} 500$ \\
\hline MAZDA & 0 & $5^{\prime} 400$ & $27^{\prime} 600$ & $93^{\prime} 300$ & $8^{\prime} 700$ \\
\hline MITSUBISHI & 0 & $23^{\prime} 300$ & $118^{\prime} 400$ & 399'500 & $37^{\prime} 500$ \\
\hline TATA & 0 & $2^{\prime} 600$ & $13^{\prime} 000$ & $43^{\prime} 800$ & $4^{\prime} 200$ \\
\hline FUلl (Subaru) & 0 & $2^{\prime} 600$ & $13^{\prime} 300$ & $45^{\prime} 000$ & $4^{\prime} 200$ \\
\hline AVTOVAZ & 0 & $2^{\prime} 400$ & $12 ' 200$ & $41 ' 300$ & $3^{\prime} 900$ \\
\hline Mahindra & 0 & 700 & 3'700 & $12^{\prime} 600$ & 1'100 \\
\hline Proton & 0 & 600 & $3^{\prime} 200$ & $10^{\prime} 800$ & $1^{\prime} 000$ \\
\hline Kuozui & 0 & 500 & $2^{\prime} 600$ & $8^{\prime} 700$ & 800 \\
\hline Porsche & 0 & 400 & 2100 & $7^{\prime} 200$ & 700 \\
\hline REST & 0 & $6 ' 300$ & $31^{\prime} 900$ & $107^{\prime} 700$ & $10^{\prime} 100$ \\
\hline Summe & 0 & 1'657'800 & $8^{\prime} 422^{\prime} 700$ & $28^{\prime} 418^{\prime} 900$ & $2^{\prime} 667^{\prime} 400$ \\
\hline \% Gesamtkap. & $0 \%$ & $2 \%$ & $11 \%$ & $31 \%$ & $1 \%$ \\
\hline
\end{tabular}

Tabelle 9: $\quad$ Modellierung der maximal möglichen Produktion von EV weltweit bis 2025 und prozentualer Anteil an der weltweiten Gesamtproduktionskapazität.

Die so erhaltene maximale weltweite Produktionskapazität für EV soll als obere Grenze gelten. Nachstehende Abbildung 9 zeigt, welche möglichen Verläufe der EV-Marktpenetration oberhalb dieser maximalen Produktionskapazität liegen (gestrichelte Verläufe). Wir betrachten diese als weniger plausibel. 


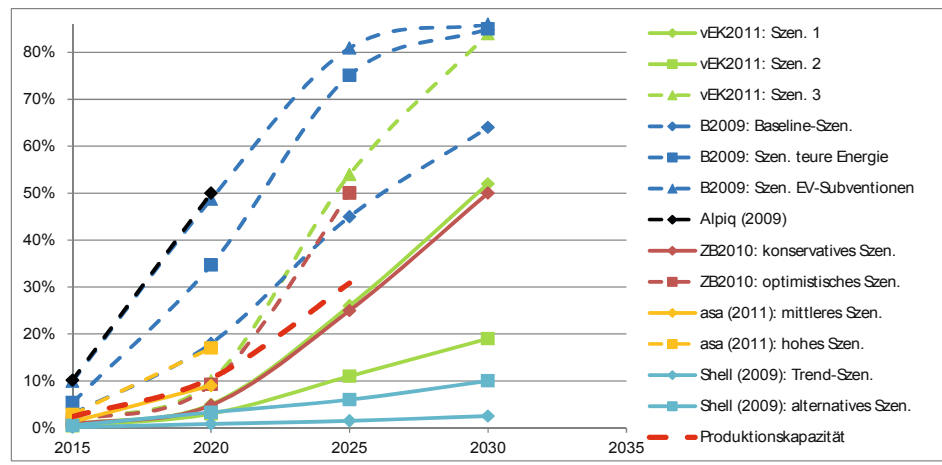

Abbildung 9: Wie Abbildung 8, ergänzt um die abgeschätzte maximale globale Produktionskapazität; Verläufe, welche diese Produktionskapazität überschreiten, werden gestrichelt eingezeichnet.

\subsection{Mikrosimulation der Elektromobilmarktanteile bis 2035}

\section{Methode und verwendetes Modell}

Die tragende Säule unserer Vorhersage der Marktpenetration von Elektromobilen in den Schweizer Automarkt bildet eine sogenannte Mikrosimulation: Für jedes Kalenderjahr bis 2035 wird für jedes Szenario eine «Angebotsflotte» erstellt. Aus dieser Angebotsflotte werden dann die neuen PKW gekauft, durch eine synthetische Bevölkerung. Diese synthetische Bevölkerung besitzt bereits Autos, und zwar für das Ausgangsjahr 2010 den Bestand der PKW in der Schweiz per 1. 9. 2009. Welches Auto ein Haushalt besitzt, hat einen Einfluss auf die Auswahlwahrscheinlichkeiten für mögliche Nachfolgemodelle, denn das Modell berücksichtigt Treueraten für Autogrössenklassen, Automarken, Treibstofftypen und Getriebearten. Auch werden, für die deutsch-, französisch- und italienischsprachigen Landesteile unterschiedliche, «Heimmarken» unterschieden, für welche eine höhere Kaufpräferenz existiert. Die synthetische Bevölkerung entspricht ansonsten den Eckwerten der Volkszählung 2000. Für andere Grenzwertverteilungen werden die Resultate einer ETHZ-Grossbefragung von 2005 verwendet: In wie vielen Fällen ein Mann oder eine Frau für den Neuwagenkaufentscheid verantwortlich zeichnet. Jeder Haushalt wird charakterisiert durch das Ge- 
schlecht, das Alter, die Einkommensklasse und den Haushalttyp (Single bei Eltern lebend; Single; Paar; Familie). Dies führt zu 40 verschiedenen Käufertypen mit je einzelnen Kaufparametern. Simuliert werden eine Million Neuwagenkäufe (in der Schweiz werden jährlich zwischen 260000 und 340000 Neuwagen verkauft; dass wir eine Million simulieren, dient lediglich dem Zweck, auch bei kleineren Marktanteilen einzelne Angebotskonzepte darstellen zu können).

Mit der Mikrosimulation erhält man nicht nur den Anteil der Elektrofahrzeuge am Marktvolumen, sondern auch die Marktverhältnisse für jedes Segment. Damit können auch die statistischen Verteilungen der Treibstoffverbräuche, $\mathrm{CO}_{2}$-Emissionswerte usw. prognostiziert werden. Das eingesetzte Mikrosimulationsmodell, sim.car, bildet auch die Basis von Analysen für die Bundesämter für Energie, Umwelt und Strassen (betreffend Fragestellung zu Bonussystemen, «Offroader-Initiative», 130-g CO $/ \mathrm{km}$-Zielwert für PKW-Neuzulassungen usw.). Das Modell wird beschrieben in de Haan et al. (2007), wissenschaftlich ausführlicher in Mueller und de Haan (2009) sowie de Haan et al. (2009).

Die Simulation ist grundsätzlich stochastisch, die Kaufverhaltensmodelle liefern lediglich Auswahlwahrscheinlichkeiten. Wenn man die Simulation mit gleichen Ausgangsgrössen und Nutzereingaben ein zweites Mal durchführt, resultieren andere Werte. Allerdings werden die aggregierten Grössen, wenn man eine Million Neuwagenkäufe simuliert, davon nur bei hinteren Kommastellen betroffen.

Die Annahmen zur mittleren $\mathrm{CO}_{2}$-Intensität der neuzugelassenen Personenwagen entsprechen grundsätzlich jenen für die schweizerischen Energieperspektiven 2050 des BFE (BFE 2012, Kap. 4.1), es zeigen sich aber leichte Abweichungen, weil namentlich die Elektromobilität einen Einfluss auf den mittleren $\mathrm{g} \mathrm{CO}_{2}$ / km-Wert aller Neuzulassungen hat. Im Jahre 2020 beträgt der mittlere Wert der Neuzulassungen 112, 111 bzw. $101 \mathrm{~g} \mathrm{CO}_{2} / \mathrm{km}$ für das BAU-, EFF- bzw. COMSzenario. Für das Jahr 2030 betragen die Werte 89, 77 bzw. $55 \mathrm{~g} \mathrm{CO}_{2} / \mathrm{km}$ (siehe Anhang A4). Die Energiepreise wurden in den drei Szenarien im Einklang mit den schweizerischen Energieperspektiven 2050 (BFE 2012, Kap. 3.3) angenommen.

Um die Reichweitenproblematik abzubilden, wird in den Szenarien BAU und EFF davon ausgegangen, dass nur für die Hälfte der Haushalte der Kauf eines EV infrage kommt, weil dies Mehr-Auto-Haushalte sind; es verbleibt ein Fahrzeug mit konventionellem Antrieb und hoher Reichweite. Im COM-Szenario wird diese Einschränkung jedoch nicht vorgenommen, weil angenommen wird, dass in diesem Szenario längere Fahrten vermehrt über kombinierte Mobilität erfolgen und 
EV vor allem als Zubringer zu ÖV-Umsteigeknoten für die erste bzw. letzte Meile eingesetzt werden.

\section{Erstellung von PKW-Angebotsflotten}

Es werden, für die ICE- und HEV-Fahrzeuge, die PKW-Flotten aus asa (2011) verwendet. Untenstehende Tabelle zeigt die Umsetzung der Szenarien in jährlichen Änderungsraten für technische Eckdaten der Elektrofahrzeuge, um damit für jedes Kalenderjahr und jedes Szenario ergänzend das Angebot an Elektrofahrzeugen in den PKW-Angebotsflotten abzubilden.

\begin{tabular}{|c|c|c|c|c|c|}
\hline & & Fullsize & Compact & Micro & 3-Wheeler \\
\hline & Marktanteil 2015 & $15 \%$ & $62 \%$ & $23 \%$ & $0 \%$ \\
\hline BAU & Änderung/Jahr & $0 \%$ & $0 \%$ & $0 \%$ & $0 \%$ \\
\hline EFF & Änderung/Jahr & $-0,50 \%$ & $-0,60 \%$ & $+0,35 \%$ & $+0,75 \%$ \\
\hline \multirow[t]{2}{*}{ COM } & Änderung/Jahr & $-0,38 \%$ & $-0,35 \%$ & $+0,35 \%$ & $+0,38 \%$ \\
\hline & Masse $(\mathrm{kg}) 2015$ & 2200 & 1500 & 1100 & 450 \\
\hline BAU & Änderung/Jahr & $-1,00 \%$ & $-1,00 \%$ & $-1,00 \%$ & $-0,00 \%$ \\
\hline EFF & Änderung/Jahr & $-3,40 \%$ & $-3,40 \%$ & $-3,40 \%$ & $-1,40 \%$ \\
\hline \multirow[t]{2}{*}{ COM } & Änderung/Jahr & $-2,50 \%$ & $-2,50 \%$ & $-2,50 \%$ & $-1,10 \%$ \\
\hline & Preis (CHF) 2015 & $90 ‘ 000$ & $48 ‘ 000$ & $37^{\prime} 000$ & $18 ‘ 000$ \\
\hline BAU & Änderung/Jahr & $-1,00 \%$ & $-1,00 \%$ & $-1,00 \%$ & $-0,00 \%$ \\
\hline EFF & Änderung/Jahr & $-1,40 \%$ & $-1,40 \%$ & $-1,40 \%$ & $-1,40 \%$ \\
\hline COM & Änderung/Jahr & $-2,00 \%$ & $-2,00 \%$ & $-2,00 \%$ & $-2,00 \%$ \\
\hline
\end{tabular}

Tabelle 10: $\quad$ Angenommene Änderungsraten der Marktanteile, der Fahrzeugmasse und der Listenpreise für die vier ElektrofahrzeugGrössenklassen.

\section{Resultat}

Nachfolgende Abbildung 10 zeigt die Marktpenetration der Elektromobile (EV+PHEV; Anteil am Neuwagenmarkt) unserer drei Szenarien im Vergleich mit der im vorausgehenden Kapitel abgeschätzten maximalen Produktionskapazität bis 2025 und im Vergleich mit anderen Studien aus der Literatur. Es zeigt sich, dass diese drei Szenarien alle klar unterhalb der Produktionskapazität liegen und sich vor allem im Zeitraum nach 2025 unterscheiden. Abbildung 11 stellt die Verläufe der Elektromobil-Marktanteile jenen der übrigen Antriebskonzepte gegenüber. 


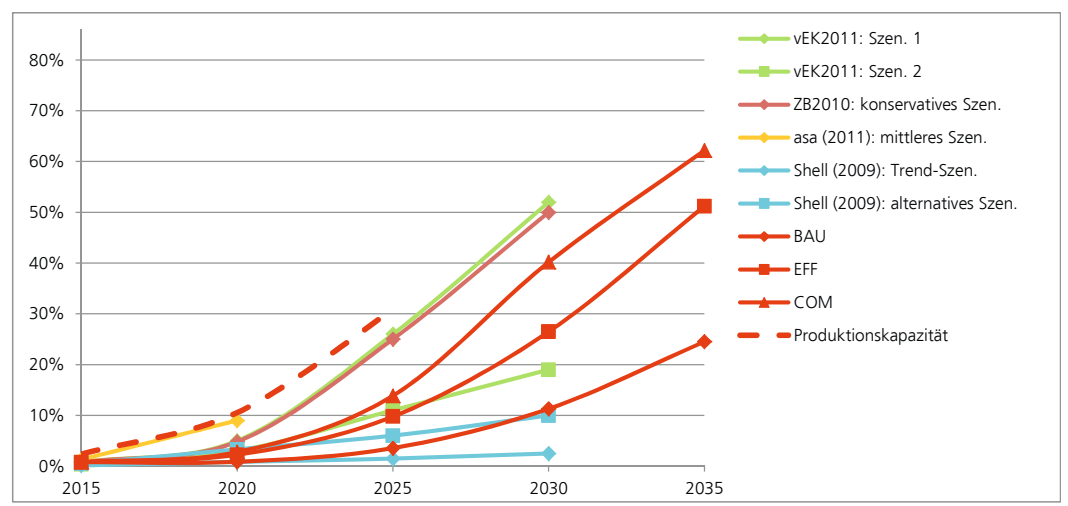

Abbildung 10: Wie Abbildung 8 (nur jene Verläufe, welche im Vergleich mit der maximalen Produktionskapazität plausibel erscheinen), ergänzt um die Verläufe der drei Szenarien der vorliegenden Studie.

\subsection{Modellierung der Elektromobilanteile 2035 bis 2050}

Die detaillierte Mikrosimulation des Automarkts macht für die langfristige Perspektive wenig Sinn. Wir verwenden deshalb ein Extrapolationsverfahren, welches aufgrund des Verlaufs der Elektromobilanteile zwischen 2015 und 2035 einen möglichst «passenden» weiteren Verlauf bis 2050 schätzt. Wir gehen dabei wie folgt vor:

- Wir verwenden die logistische Funktion; dies ist die klassische Funktionsform mit anfänglich exponentiellem Verlauf, einer anschliessenden Sättigungsphase und der Annäherung eines Endwerts, welche zur Beschreibung des Wachstums von Populationen verwendet wird;

- Wir gehen nicht davon aus, dass der finale Marktanteil von Elektromobilen 100 Prozent betragen wird, sondern weniger: Auch in 80 Jahren dürfte der Langdistanzgüterstrassentransport mit flüssigen Kohlenwasserstoffen, ähnlich dem heutigen Diesel, bewältigt werden. Ob fossilen oder biogenen Ursprungs ist unerheblich: Ein Tankstellennetz wird damit weiterhin vorhanden sein. Damit wird es auch in 80 Jahren möglich sein und Sinn machen, für Langdistanz-LNF und -PKW auch Diesel einzusetzen. Der Endwert des Elektromobilanteils 


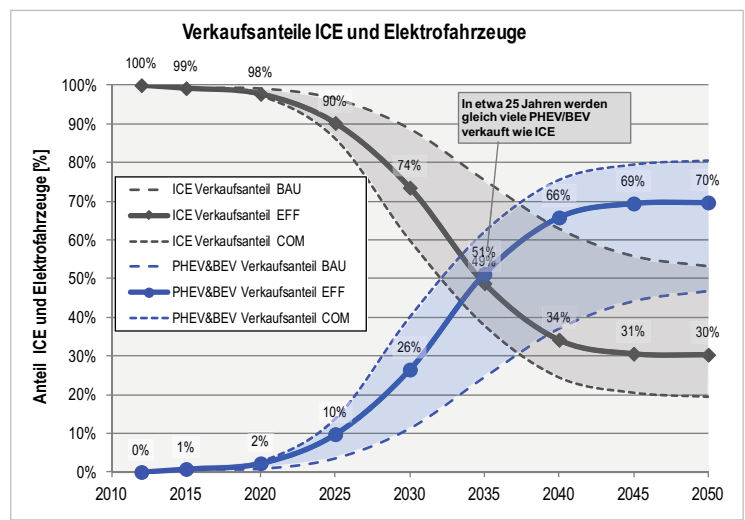

Abbildung 11: Verläufe der Marktanteile der Elektromobile (EV+PHEV) am PKWNeuwagenmarkt in den drei Szenarien der vorliegenden Studie.

hängt damit von der technischen Entwicklung, den Energiepreisen, der Energiepolitik und der Verfügbarkeit fossiler Treibstoffe ab.

- Wir verwenden die freien Parameter M (Endwert, d.h. finaler Marktanteil im Jahr 2050), $t_{s}$ (Jahr der maximalen Steigung) und a (Spreizung): Der Elektromobilmarktanteil, $\mathrm{P}$, beträgt im Jahr $\mathrm{t}$ damit $\mathrm{P}(\mathrm{t})=\mathrm{M}\left(1+\exp \left[-\left(\mathrm{t}-\mathrm{t}_{\mathrm{s}}\right) / \mathrm{a}\right]\right)^{(-1)}$.

- Die Zahlenwerte der freien Parameter werden mittels der Methode der Minimierung der Summe der Fehlerquadrate bestimmt, dabei wird die Differenz zwischen dem Funktionswert $\mathrm{P}(\mathrm{t})$ und den Resultaten aus dem vorangehenden Kapitel für die Jahre 2015, 2020, 2025, 2030 und 2035 gebildet.

Für die Szenarien BAU, EFF bzw. COM erhalten wir damit als Endwerte $M=48$ Prozent, 70 Prozent bzw. 81 Prozent; die maximalen Steigerungsraten treten im Jahr $t_{s}=2035,2032$ bzw. 2030 auf sowie a= 4,1; 3,6 bzw. 3,6. Die obenstehende Abbildung 11 zeigt die Verläufe grafisch.

\subsection{Entwicklung von Bevölkerung, Motorisierungs- grad, Fahrleistung und Fahrzeugflotte}

Die Bevölkerungsentwicklung wird dem «mittleren Szenario» des BFS (2010b) entnommen, dieses Szenario sieht ein deutliches Bevölkerungswachstum vor. Das Verkehrsaufkommen, d.h. die Gesamtzahl der im MIV zurückgelegten Personenkilometer und Fahrzeugkilometer, wird der Fortsetzung von ARE (2006) im 
Rahmen der neuen Energieperspektiven des BFE vom Herbst 2012 entnommen. Für alle drei Szenarien wird die gleiche Anzahl Fahrzeugkilometer gewählt, damit die Vergleichbarkeit gewährleistet bleibt. Allerdings wird im COM-Szenario angenommen, dass ein Teil der bisherigen PKW-Fahrleistung neu durch die Kategorie der 3-Wheeler abgedeckt werden kann. Wir analysieren mithilfe der Daten des neuen Mikrozensus Verkehr (BFS 2012), was diese Annahme für das mittlere Mobilitätsverhalten in der Schweiz bedeutet (Anhang A3). Inwiefern der vermehrter Einsatz von effizienten PKW (im EFF-Szenario) bzw. vor allem von effizienten PKW mit Elektroantrieb (im COM-Szenario) zu Reboundeffekten (mehr Fahrzeugkilometer) führen könnte, analysieren wir in Kapitel 8.

Die Annahmen zur Bevölkerungsentwicklung, zum Motorisierungsgrad sowie zur Anzahl Personenwagenkilometer pro Kopf wurden im Einklang mit den schweizerischen Energieperspektiven 2050 gewählt (BFE 2012, Kap. 7.4.4, 8.4.4 bzw. 9.4.4). Sowohl der Motorisierungsgrad (Anzahl Fahrzeuge pro 1000 ständig in der Schweiz wohnhaften Personen) als auch die Fahrzeugkilometer nehmen dabei zu, allerdings nimmt die Anzahl Fahrzeugkilometer pro Auto leicht ab.

Für die Modellierung der Fahrzeugflotte wird ein Flottenmodell verwendet: Jährlich kommen die Neuzulassungen hinzu, während ein Teil der älteren Flotte ausscheidet. Dies wird über sogenannte Überlebensraten je Kohorte simuliert. In Abhängigkeit von der Fahrzeuggrössenklasse sowie dem Alter wird jedes Jahr ein Teil jeder Kohorte ausser Dienst gesetzt. Jährlich werden etwa 7,5 Prozent des Gesamtbestandes ersetzt. Damit dauert es länger als zehn Jahre, bis der Bestand umgewälzt wird; erst nach mindestens fünf Jahren zeigen sich technologische Trendbrüche im Gesamtbestand deutlich.

\subsection{Entwicklung des Energiesystems (inkl. Strommix)}

Für die Entwicklung des Energie- und namentlich des Stromsystems werden die Ergebnisse der Energiestrategie 2050 des Bundesrates verwendet:

- Für alle drei Szenarien wird die gleiche zeitliche Entwicklung des Strommixes unterlegt (siehe Kapitel 5.3);

- In einer Sensitivitätsanalyse untersuchen wir, welchen Einfluss Variationen im Strommix auf das Gesamtresultat haben können (Kapitel 5.6.2). 


\section{Zukünftige Entwicklung der Fahr- zeugkomponenten}

\subsection{Einführung}

Elektrische Mobilität steht heute an der Schwelle zum Massenmarkt - der vollelektrische Nissan Leaf gehört bereits heute zu den 25 meist verkauften Fahrzeugmodellen. Ob und wie der breite Markteintritt gelingen wird, hängt stark von der technischen und ökonomischen Weiterentwicklung einzelner Schlüsselkomponenten ab. Obwohl die technischen Grundlagen weitgehend bekannt sind, bleibt unklar, wie diese Faktoren in Zukunft zusammenspielen und damit den Erfolg oder Misserfolg der Elektromobilität beeinflussen werden. Ziel dieses Kapitels ist es daher, mögliche Wege der zukünftigen Entwicklung von Elektrofahrzeugen und deren wichtigsten Komponenten aufzuzeigen und damit die Basis für die anschliessende Nachhaltigkeitsbeurteilung (Kapitel 7) zu legen.

Aktuelle und potenziell relevante Technologien für die Elektromobilität werden hierbei im Hinblick auf Effizienz und Marktpotenzial untersucht. Im Fokus stehen die Komponenten des elektrischen Antriebsstrangs: Batterie, Elektromotor und Leistungselektronik. Ebenfalls betrachtet wird das Off-Board-Ladesystem, welches einen wichtigen Teil der Energieversorgungskette darstellt. Darüber hinaus wird die künftige Entwicklung weiterer Faktoren analysiert, die für die Fahrzeugeffizienz wichtig sein können, wie Rollwiderstand, Gesamtgewicht, Aerodynamik oder das Heiz- und Kühlsystem. Schliesslich diskutieren wir die Technologieund Marktentwicklung auf der Stufe Gesamtfahrzeug.

Der aktuelle Stand der Technik (2012) basiert auf Fahrzeugen, die derzeit am Markt erhältlich sind. Die technologische Entwicklung wird für die kurzfristige (2020) und die mittelfristige Perspektive (2035) diskutiert und ein langfristiger Ausblick auf die mögliche Entwicklung bis zum Jahr 2050 gegeben. Da die Weiterentwicklung der Technologie natürlich nicht auf Elektrofahrzeuge beschränkt ist, wird zwischen Fahrzeugen mit Verbrennungsmotor (ICE), Plug-In-HybridElektrofahrzeugen (PHEV) und rein batteriebetriebenen Elektrofahrzeugen (BEV) unterschieden. Die Abschätzung für die verschiedenen Antriebssysteme wird nach den gleichen Prinzipien und im gleichen Detaillierungsgrad vorgenommen, um die Vergleichbarkeit der Ergebnisse zu gewährleisten. 


\subsection{Batterien}

Batterien sind derzeit der einzige auf dem Markt erhältliche Energiespeicher für Elektrofahrzeuge (siehe auch Box 1). Die Batterie ist die teuerste Komponente eines Elektrofahrzeugs und damit - zusammen mit der beschränkten Reichweite - wohl der wichtigste limitierende Faktor für die Penetration von Elektromobilität. Die Entwicklung von Kosten und Leistung der Batterien bestimmt die zukünftige Verbreitung von Elektrofahrzeugen daher wesentlich mit. Dieses Kapitel beleuchtet deshalb aktuelle und potenzielle Batterietechnologien und deren zukünftiges Entwicklungspotenzial. Es basiert im Wesentlichen auf einer kürzlich veröffentlichten Studie der CE Delft zur zukünftigen Entwicklung von Elektrofahrzeugen in Europa (Duleep et al. 2011) und wurde ergänzt durch weitere Studien und aktuelle Informationen der Industrie.

\section{Box 1: Wie funktioniert eine Batterie?}

Der Term Batterie bezeichnet elektrochemische Zellen, welche gespeicherte chemische Energie direkt in Elektrizität konvertieren können. Primärbatterien transformieren chemische Energie irreversibel in Elektrizität, bei Sekundärbatterien kann der Prozess umgekehrt werden (Wiederaufladung). Eine Batterie besteht aus mehreren einzelnen Zellen, welche die Basiselemente Anode (negative Elektrode), Kathode (positive Elektrode) und Elektrolyt beinhalten.

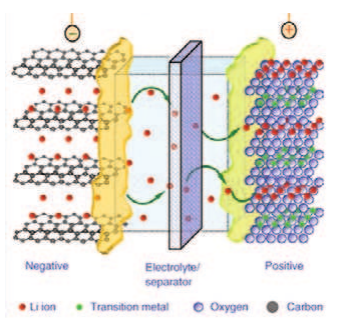

Bei der Redoxreaktion in der Batterie werden beim Entladen auf der Kathodenseite Kationen reduziert (Elektronen hinzugefügt) und auf der Anodenseite Anionen oxidiert (Elektronen weggenommen). Der Prozess kehrt sich beim Laden um. Die Elektroden werden durch einen Separator isoliert, welcher für lonen aus dem Elektrolyten durchlässig ist.

Neben der Batteriespannung gibt es weitere wichtige Indikatoren, die für die Leistung eines Elektrofahrzeugs wichtig sind: Energiedichte, Batteriekapazität, Haltbarkeit und Kosten.

Energiedichte: Gespeicherte Energie pro Masse oder Volumen.

Einheit: Wh/kg oder Wh/l.

Kapazität: In der Batterie gespeicherte Ladung. Äquivalent zum Strom, den eine Batterie bis zur vollen Entladung in einer Stunde liefern kann.

Einheit: Ampèrestunden (Ah).

\section{Haltbarkeit:}

- Kalendarische Lebensdauer: Zeitdauer, bis die Kapazität auf 80 Prozent des Anfangswerts gesunken ist.

- Zyklenzahl («Cycle Life»): Anzahl volle Lade-/Entladezyklen, bis die Kapazität auf 80 Prozent des Anfangswerts gesunken ist.

- Temperaturbereich: Bereich, dem eine Batterie bei der Lagerung oder im Betrieb ausgesetzt werden kann. 


\subsubsection{Gegenwärtiger Stand der Technologie}

Unterschiedliche Batterietypen finden gegenwärtig in EV Verwendung: LithiumIonen (Li-lon), Nickel-Metall-Hybrid (NiMH), Natrium-Nickel-Chlorid (Zebra) und Bleisäure sind die gebräuchlichsten Batterietechnologien. Die Lithium-IonenBatterie ist jedoch dabei, sich im Mobilitätsbereich aufgrund ihrer hohen Energiedichte und ihrem grossen Entwicklungspotenzial durchzusetzen.

\section{Lithium-lonen-Technologie}

Unterschiedliche Batterietechnologien werden unter dem Oberbegriff «LithiumIonen-Batterie» zusammengefasst. Während gegenwärtig alle kommerziellen Li-Ion-Batterien Graphit-Anoden besitzen, variiert die Zusammensetzung der Kathode. Eisen-Phosphat-Kathoden gelten als sicherste und günstigste Technologie, weisen aber niedrigere Energiedichten auf. Nickel- und Kobalt-Kathoden zeigen hohe Energiedichten, sind aber weniger sicher und weniger dauerhaft, während die Eigenschaften von Mangan-Kathoden dazwischen liegen.

Im Handel erhältliche Lithium-Ionen-Batterien haben eine Energiedichte auf dem Zellniveau von 130 bis $160 \mathrm{Wh} / \mathrm{kg}$. Dies entspricht etwa einem Drittel der theoretischen Grenze von 400 bis $500 \mathrm{Wh} / \mathrm{kg}$, ist aber wenig im Vergleich zur Energiedichte von Benzin (11 $000 \mathrm{Wh} / \mathrm{kg}$ ). Durch die zusätzliche Verpackung, Verdrahtung und Steuerelektronik reduziert sich die durchschnittliche Energiedichte auf Stufe Batterie auf 80 bis $110 \mathrm{Wh} / \mathrm{kg}$, was etwa 35 bis 40 Prozent niedriger ist als auf der Zellstufe. Gemäss einer Analyse von Grünig, Witte et al. (2011) betrug die durchschnittliche Kapazität von Batterien der betrachteten Elektrofahrzeuge (vorwiegend Concept Cars) $23 \mathrm{kWh}$, wobei BEV-Batterien eine Kapazität zwischen 10 und $30 \mathrm{kWh}$ bei einem durchschnittlichen Batteriegewicht von 299 Kilo und PHEV-Batterien eine Kapazität zwischen 10 und 20 kWh und ein durchschnittliches Batteriegewicht von 145 Kilo aufwiesen.

Der grundsätzliche Aufbau einer Lithium-lonen-Batterie ist bei allen etablierten Technologien sehr ähnlich, die Unterschiede liegen vorwiegend in der Zusammensetzung des Kathodenmaterials. Die Technologien weisen etwas unterschiedliche Vor- und Nachteile auf. Beispielsweise gelten Batterien mit LithiumEisenphosphat-Kathode als sicherer als cobalthaltige Batterien, welche dafür Vorteile bei der Energiedichte aufweisen. 
Das Thema Sicherheit von Li-lonen-Batterien wird nach spektakulären Fällen von Selbstentzündungen vor allem im Bereich der Unterhaltungselektronik kontrovers diskutiert. Moderne Fahrzeugbatterien werden zwar rigoros geprüft (Kurzschlussfestigkeit, Durchstich, Crash etc.) und eine Selbstentzündung kann weitgehend ausgeschlossen werden. Trotzdem bestehen Batterien aus brennbaren Materialien und können bei einem normalen Fahrzeugbrand auch indirekt entzündet werden. Bei allen heutigen Batterietypen können dabei toxische Emissionen (fluorhaltige Verbindungen) beim Abbrennen des Elektrolyten entstehen.

Folgende Abbildung veranschaulicht spezifische Unterschiede verschiedener Batterietypen:

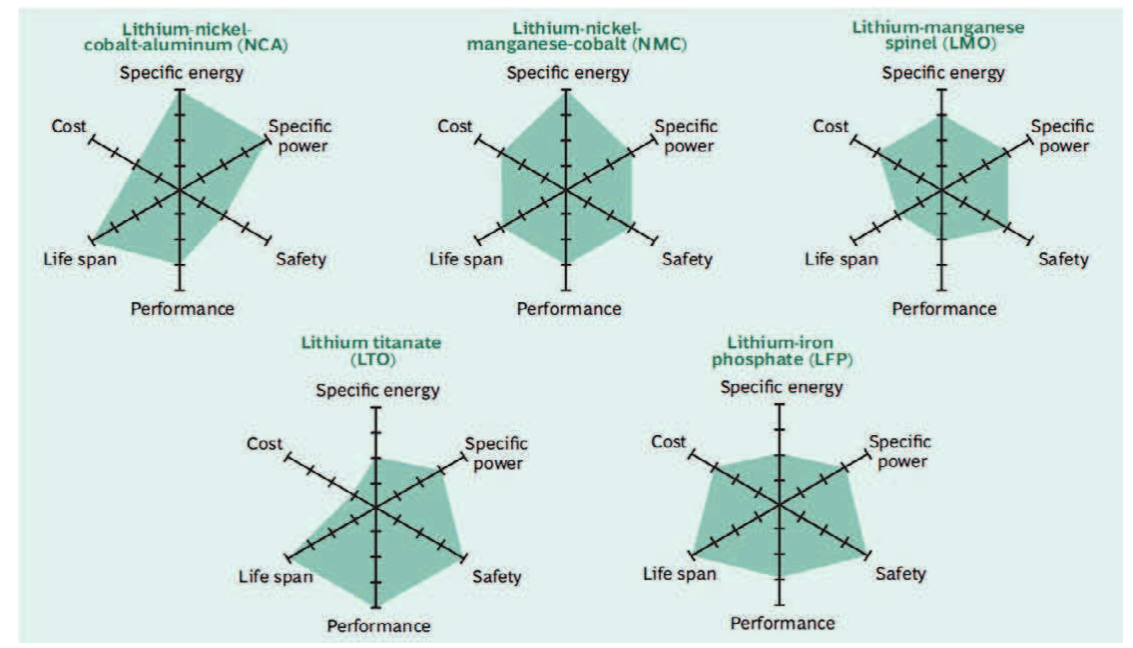

Abbildung 12: Veranschaulichung der spezifischen Eigenschaften von fünf gängigen Li-Ionen-Batterietypen. Je grösser die dunkle Fläche, desto besser die Eigenschaften bezüglich des entsprechenden Indikators. Grafikquelle: BCG (2010).

Alterung und Ladefähigkeit der Batterien sind zentrale Faktoren für den Erfolg der Elektromobilität. Die Zielwerte für 80 Prozent Batterieleistung liegen bei:

- 12 Jahre kalendarische Lebensdauer bei einer Temperatur von $35^{\circ} \mathrm{C}$

- 5000 vollständige Ladezyklen 
- 200000 bis 300000 Teilladezyklen (wichtig für Hybridfahrzeuge, bei denen die Batterie ständig aufgeladen und entladen wird)

- Arbeitsbereich von $-40^{\circ} \mathrm{C}$ bis $+66^{\circ} \mathrm{C}$ (ohne zusätzliche Batterieklimatisierung)

Inwieweit diese Zielgrössen von den heute verwendeten Batterien erfüllt werden, ist schwierig zu sagen. Einige der heute am Markt erhältlichen Batterien dürften die geforderten Ladezyklen bei Normalladung (keine Schnellladung) bereits erfüllen, während das gegenwärtige Batteriealter bei etwa zehn Jahren liegt und es noch wenig Informationen zu den Auswirkungen von Extremtemperaturen gibt. Deshalb wird heute häufig eine Batterieheizung und -kühlung vorgesehen, was den Energieverbrauch des Fahrzeugs erhöht. Mittelfristig sollte eine Batterie alle Zielgrössen erreichen, um kommerziell erfolgreich sein zu können.

Der Energiebedarf und die Treibhausgasemissionen aus der Batterieherstellung werden gegenwärtig kontrovers diskutiert. Die zurzeit umfangreichste und transparent dokumentierte Studie von Notter et al. (Notter, Gauch et al. 2010) weist Treibhausgasemissionen von $6,0 \mathrm{~kg} \mathrm{CO}$ eq $/ \mathrm{kg}$ Batterie aus (basierend auf dem europäischen Strommix). Andere Studien kommen dagegen auf bis zu viermal höhere Werte (Zackrisson, Avellán et al. 2010; Frischknecht 2012). Eine neue amerikanische Studie (Dunn et al. 2012) bestätigt in einer detaillierten Analyse die Werte von Notter et al. und zeigt als Treibhausgasemission 5,1 kg $\mathrm{CO}_{2} \mathrm{eq} / \mathrm{kg}$ Batterie.

Bei den Kostenschätzungen für Li-lon-Batterien gibt es grosse Unterschiede zwischen Zell- und Batterieniveau. Boston Consulting Group (BCG 2010) schätzt die Zellkosten auf 720 bis 770 US $\$ / k W h$ und die Batteriekosten auf 1000 bis 1110 US $\$ / k W h$. CE Delft (Duleep et al. 2011) schätzt für das Jahr 2012 die nicht subventionierten Zellkosten auf 500 US\$/kWh und die entsprechenden Batteriekosten auf 750 bis 800 US $\$ / k W h$.

\section{Andere Batterietechnologien}

Obwohl Li-lon-Batterien den Markt immer stärker dominieren, hat die SalzSchmelz-Technologie (z.B. ZEBRA-Batterie) auch eine gewisse Bedeutung erlangt. Diese Technologie ermöglicht eine hohe Energiedichte und Zuverlässigkeit bei niedrigen Herstellungskosten, da die Batterien auf breit erhältlichen 
Komponenten beruhen. Der Hauptnachteil ist aber die hohe Betriebstemperatur von $300^{\circ} \mathrm{C}$, was einen konstanten Stromverbrauch von 60 bis $80 \mathrm{~W}$ verursacht, auch wenn das Auto nicht fährt. Ein grosser Vorteil der ZEBRA-Batterie ist hingegen die hohe Betriebssicherheit (Eigensicherheit).

\subsubsection{Generelle Technologieentwicklung}

Die erste Generation von Li-Ion-Batterien wurde erst 2011/2012 im Elektromobilmarkt eingeführt. Die Produktionsdauer einer Li-lon-Generation beträgt rund vier bis fünf Jahre, um Investitionen und F\&E-Kosten zu decken. Entwicklungsziel für die nächste Generation ist primär die Erhöhung der Energiedichte und die Reduktion der Herstellungskosten bei Aufrechterhaltung einer Ladezyklenzahl von mehr als 2000 und eines Batteriealters von mehr als 10 Jahren. Die vielversprechendsten neuen Lithiumchemien beruhen auf Silizium, Schwefel und Luft (Sauerstoff). Die Nanostrukturierung der Elektrodenoberflächen erlaubt eine weitere Erhöhung der Energiedichte und der Ladestabilität.

\section{Lithium-Silizium-Anoden}

Nanostrukturiertes Silizium ist ein attraktives Anodenmaterial als Ersatz für Graphit-Anoden, da die theoretische Kapazität von $4000 \mathrm{mAh} / \mathrm{g}$ zehnmal höher liegt als bei Graphit. Zusätzlich ist Silizium breit verfügbar, was die Produktionskosten niedrig hält. Das Hauptproblem liegt bei einer Volumenänderung von 400 Prozent während der Lade-/Entladezyklen, was die Anwendung in aktuellen Batteriedesigns verhindert. Prototypen von nanostrukturierten Silizium-Anoden der ersten Generation, die von Nexeon entwickelt wurden, haben eine Kapazität von $1000 \mathrm{mAh} / \mathrm{g}$, währenddem die zweite Anodengeneration bis zu $3600 \mathrm{mAh} / \mathrm{g}$ haben soll. Beide liessen sich in konventionellen Batteriesystemen integrieren, der Nutzen ist jedoch beschränkt, solange die Kathoden keine höhere Kapazität als 100 bis $300 \mathrm{mAh} / \mathrm{g}$ aufweisen.

\section{Lithium-Schwefel-Kathoden}

Schwefelbasierte Kathoden weisen eine theoretische Kapazität von 1600 mAh/g auf, viel höher als die Kapazitäten der gegenwärtigen Metalloxid- und PhosphatKathoden von $300 \mathrm{mAh} / \mathrm{g}$. Diese höhere Kapazität erlaubt fünfmal höhere Energiedichten als konventionelle Li-lon-Batterien. Probleme bereiten aber noch signifikante strukturelle Veränderungen während der Lade-/Entladezyklen und die 
Auflösung der Lithium-Polysulfide im Elektrolyten. Sion (2011) gibt an, eine Prototypzelle mit einer spezifischen Energiedichte von mehr als $350 \mathrm{Wh} / \mathrm{kg}$ entwickelt zu haben und in der nahen Zukunft $600 \mathrm{Wh} / \mathrm{kg}$ erreichen zu wollen.

\section{Lithium-Luft}

Diese Technologie basiert auf Sauerstoff als katalytische Luft-Kathode. Im Vergleich zu allen anderen Li-lonen-Technologien bietet die Li-Luft-Technologie wesentliche Vorteile. Mit Sauerstoff als unlimitiertem aktivem Kathodenmaterial wird die Batteriekapazität nur durch die Anode begrenzt. Da die Kathode üblicherweise die teuerste Komponente ist, beinhaltet die Li-Luft-Technologie auch ein sehr grosses Sparpotenzial. Mit einer theoretischen Energiedichte von $13000 \mathrm{Wh} / \mathrm{kg}$ ist es die einzige Batterietechnologie, mit der sich potenziell Energiedichten im Bereich der flüssigen Kohlenwasserstoffe erzielen lassen.

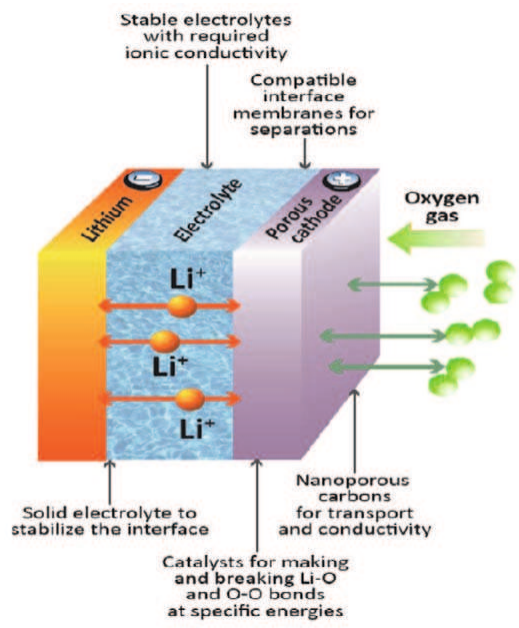

Abbildung 13: Diagramm einer Lithium-Luft-Zelle (Quelle: Duleep et al. 2011).

Die Technologieentwicklung befindet sich jedoch noch in einem experimentellen Laborstadium und viele Herausforderungen müssen gemeistert werden. Zum Beispiel sind die Sauerstoffdiffusionsraten in die poröse Kathode noch viel zu niedrig, und es entstehen dabei Verkrustungen, die den Kontakt zwischen Luft und Elektrolyte reduzieren. Zusätzlich können kleinste Verunreinigungen durch Wasser zur Bildung von Wasserstoff und akuter Explosionsgefahr führen. PolyPlus (2011) meldet jedoch, dass ihre Li-Luft-Zelle für die Anwendung in Sensoren 
kurz vor der Kommerzialisierung steht und bereits Energiedichten von $700 \mathrm{Wh} /$ kg erreicht.

Eine Publikation in Nature (Jung et al. 2012) nennt eine theoretische Energiedichte der Elektrode von $13500 \mathrm{Wh} / \mathrm{kg}$ und stabiles Verhalten über 100 Zyklen unter Verwendung eines speziellen Elektrolytmaterials. Bei einer beschriebenen Reduktion der Energiedichte um eine Grössenordnung durch die Nebenkomponenten resultieren so $1300 \mathrm{Wh} / \mathrm{kg}$ auf Batterieniveau.

\subsubsection{Entwicklungspfade}

Gemäss Duleep et al. (2011) können die folgenden Entwicklungen in der Batterietechnologie erwartet werden:

- 20 bis 25 Prozent Erhöhung der spezifischen Energiedichte bei ähnlicher Kostenreduktion bis 2016, erzielt durch optimiertes Batteriedesign und -verpackung

- 70 bis 75 Prozent Erhöhung der spezifischen Energiedichte und 50 Prozent Kostenreduktion (pro kWh) bis 2020/2022 mit der Markteinführung von siliziumbasierten Anoden-Materialien

- Potenzial für eine Verdreifachung der Energiedichte und 70 Prozent Kostenreduktion (pro kWh) bis 2030 mit der Einführung von Li-Schwefel-Kathoden

- Verlängerung der Batterielebensdauer von gegenwärtig zehn Jahren auf 13 bis 15 Jahre bis 2020

Li-Luft wurde bei der Abschätzung des Verbesserungspotenzials nicht berücksichtigt, da die Kommerzialisierung für portable IT-Geräte mindestens 15 bis 20 Jahre und für Fahrzeugbatterien 25 bis 30 Jahre dauern dürfte.

\section{Entwicklung der Energiedichte}

Abbildung 14 zeigt die von Duleep et al. (2011) prognostizierte Entwicklung der Energiedichte auf Stufe Batterie (inklusive Gehäuse, Kabel und Überwachungselektronik) und stellt sie anderen Prognosen und der vergangenen Entwicklung von Li-Ion-Zellen für den ICT-Sektor gegenüber. Die Entwicklung der Energie- 
dichte von Li-lon-Zellen im ICT-Bereich hat zu einer heutigen Energiedichte von rund $250 \mathrm{Wh} / \mathrm{kg}$ geführt; dies ist etwa doppelt so viel wie bei heutigen Li-lonBatteriesystemen für Fahrzeuge. Die zukünftige Entwicklung der Energiedichte hängt einerseits von der kontinuierlichen Verbesserung der Herstellungsprozesse ab, andererseits von der Marktpenetration neuer Technologien (wie z.B. siliziumbasiertes Anodenmaterial). Die diesbezügliche Schätzung des Entwicklungspotenzials in der Roadmapstudie des Fraunhofer-ISI-Instituts (Fraunhofer 2011) deckt sich mit der Prognose von Duleep et al. (2011). Konkrete Schätzungen der zukünftigen, am Markt erhältlichen Energiedichten sind dennoch mit hohen Unsicherheiten verbunden (Abbildung 14, grauer Unsicherheitsbereich).

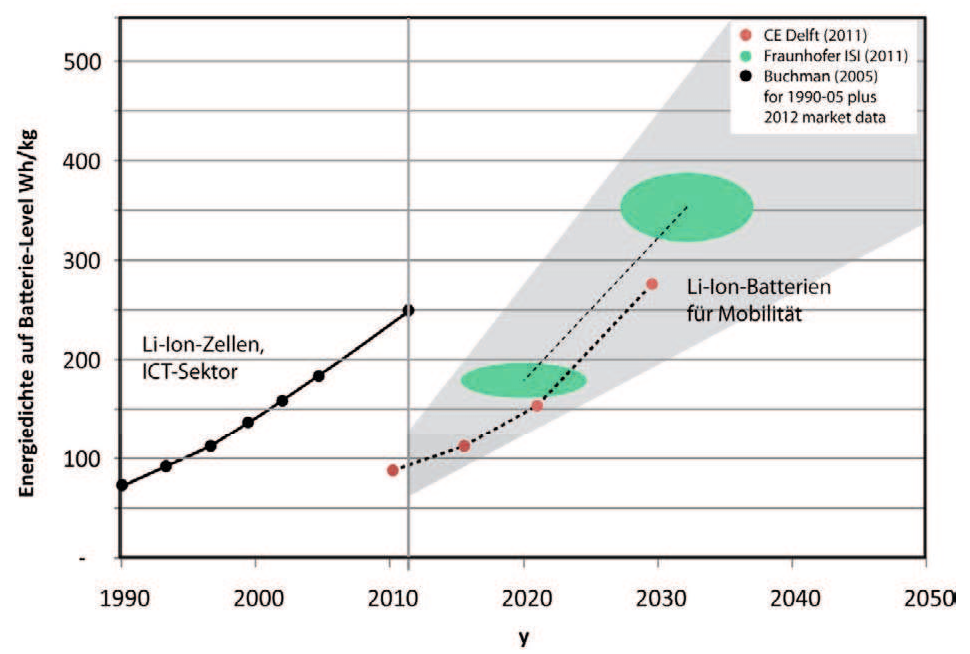

Abbildung 14: Vergangene Entwicklung der Energiedichte von Li-lon-Zellen und prognostizierte Entwicklungstrends für Li-Ion-Batterien für den Mobilitätseinsatz.

\section{Entwicklung der Kosten}

Abbildung 15 zeigt die von CE Delft prognostizierte Entwicklung der Kosten auf Stufe Batterie und stellt sie anderen Prognosen und der vergangenen Entwicklung von Li-lon-Zellen im ICT-Sektor gegenüber. Die Kosten für Li-lon-Zellen im ICT-Bereich sind über die letzten 20 Jahre signifikant gesunken. Auch die Kosten für Li-lon-Batterien für den Mobilitätssektor haben sich in den Jahren seit ihrer 
Markteinführung bereits deutlich vermindert. Generell zeigen die vier untersuchten Marktstudien ähnliche Kosten-Reduktionsraten auf, starten aber auf verschiedenen Preisniveaus, was auf die hohe Varianz der Batteriekosten hinweist.

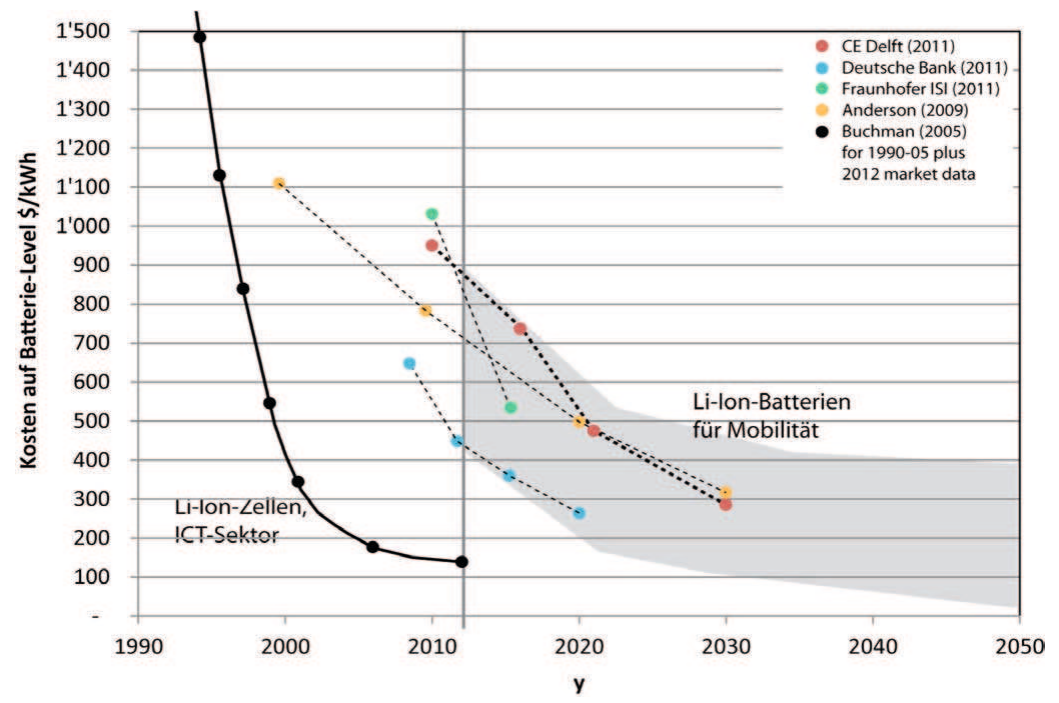

Abbildung 15: Vergangene Entwicklung der Kosten von Li-lon-Zellen und prognostizierte Entwicklungstrends für Li-lon-Batterien für den Mobilitätseinsatz.

In einer aktuellen Pressemitteilung (Envia 2012) wird sogar behauptet, $400 \mathrm{Wh} /$ $\mathrm{kg}$ auf Zellniveau zu einem geschätzten Preis von USD 125/kWh bereits erreicht zu haben. Diese Werte würden den Prognosen für 2030 entsprechen.

Generell zeigen sich grosse Unsicherheiten in der Prognose der Li-lon-Batterieentwicklung. In dieser Studie folgen wir daher den eher konservativen Werten der umfassenden Technologieentwicklungsstudie von Duleep et al. (2011) und ergänzen diese durch eine Unsicherheitsanalyse der Ökobilanz für die Jahre 2035 und 2050.

\subsubsection{Annahmen der Studie für die Batterieentwicklung}

In Tabelle 11 finden sich die aus der Technologieanalyse abgeleiteten Faktoren für die zukünftige Entwicklung der Li-Ion-Batterien für die Elektromobilität. Die 
Angaben beziehen sich auf Kompaktwagen. Die entsprechenden Angaben für die anderen Fahrzeugklassen finden sich im Anhang A5.

\begin{tabular}{|c|c|c|c|c|c|c|}
\hline Faktor & Einheit & & 2012 & 2020 & 2035 & 2050 \\
\hline \multirow[t]{3}{*}{ Batteriemasse } & \multirow[t]{3}{*}{$\mathrm{kg}$} & ICE/HEV & - & - & - & - \\
\hline & & PHEV & 100 & 100 & 100 & 100 \\
\hline & & BEV & 300 & 300 & 300 & 300 \\
\hline \multirow{3}{*}{$\begin{array}{l}\text { Spezifische } \\
\text { Energiedichte }\end{array}$} & \multirow[t]{3}{*}{$\mathrm{Wh} / \mathrm{kg}$} & ICE/HEV & - & - & - & - \\
\hline & & PHEV & 100 & 154 & 313 & 360 \\
\hline & & BEV & 100 & 154 & 313 & 360 \\
\hline \multirow{3}{*}{$\begin{array}{l}\text { Batteriekapazität } \\
(100 \%)\end{array}$} & \multirow[t]{3}{*}{$\mathrm{kWh}$} & 0 & - & - & - & - \\
\hline & & PHEV & 10 & 15 & 31 & 36 \\
\hline & & BEV & 30 & 46 & 94 & 108 \\
\hline \multirow{3}{*}{$\begin{array}{l}\text { Batteriewirkungsgrad } \\
\text { (ohne Ladegerät) }\end{array}$} & \multirow[t]{3}{*}{$\%$} & ICE/HEV & $0 \%$ & $0 \%$ & $0 \%$ & $0 \%$ \\
\hline & & PHEV & $92 \%$ & $94 \%$ & $95 \%$ & $96 \%$ \\
\hline & & BEV & $92 \%$ & $94 \%$ & $95 \%$ & $96 \%$ \\
\hline \multirow[t]{3}{*}{ Lebensdauer Batterie } & \multirow[t]{3}{*}{$\mathrm{km}$} & ICE/HEV & - & - & - & - \\
\hline & & PHEV & $100^{\prime} 000$ & $150 ' 000$ & $150^{\prime} 000$ & $150^{\prime} 000$ \\
\hline & & BEV & $100^{\prime} 000$ & $150 ' 000$ & $1500^{\prime} 000$ & $150^{\prime} 000$ \\
\hline
\end{tabular}

Tabelle 11: $\quad$ Getroffene Annahmen für die zukünftige Batterieentwicklung für Fahrzeuge der Kompaktklasse.

Die Batteriemasse wird für die Zukunft als konstant angenommen. Bei konstanter Batteriemasse und steigender Energiedichte ergeben sich damit grössere Reichweiten. Die Batteriemasse für BEV liegt gegenwärtig bei zirka 300 Kilo (Nissan Leaf, Renault Fluence) und für PHEVs bei zirka 100 Kilo (Chevrolet Volt rund 200 Kilo für eine Batteriekapazität von $16 \mathrm{kWh}$ ). Es bestehen Unterschiede im Einsatz der Batterie (und den Anforderungen an die Batterie) zwischen HEV und $B E V$, diese Unterschiede führen dazu, dass die theoretische Kapazität teilweise nie ausgeschöpft wird: HEV fahren im «Charge Sustaining Mode» mit einem State-Of-Charge (SOC) von 30 bis 70 Prozent, während BEV im «Charge Depleting Mode» den SOC von anfänglich 100 Prozent auf beinahe null Prozent reduzieren. PHEV nehmen hier eine Zwischenstellung ein, verhalten sich anfänglich wie BEV, bei längeren Fahrten wie HEV.

Bei der spezifischen Energiedichte stützen wir uns auf die Schätzungen der CEDelft-Studie (Duleep et al. 2011) ab, welche eher konservativer sind als die Schätzungen des Fraunhofer Instituts (Fraunhofer 2011), ergänzt durch eine konservative Annahme für 2050 von $350 \mathrm{Wh} / \mathrm{kg}$ (siehe auch Abbildung 14). 
Die Batteriekapazität ergibt sich aus dem Produkt des Batteriegewichts mit der spezifischen Energiedichte und bezeichnet die maximal entnehmbare Energie bei hundertprozentiger Entladung. Heute wird diese theoretische Kapazität von einigen Herstellern begrenzt (z.B. auf 30 bis 80 Prozent statt null bis 100 Prozent), wodurch die Lebensdauer der Batterien verlängert werden kann zulasten der Speicherkapazität, welche wiederum die Reichweite definiert.

Der Batteriewirkungsgrad zeigt das Verhältnis von Entlade- zu Ladeenergie. Ein Teil der Ladeenergie wird in Wärme umgewandelt, sodass beim Entladen nicht die gesamte ursprünglich geladene Energiemenge zur Verfügung steht. Blei-Akkus weisen Wirkungsgrade von rund 75 Prozent auf, während sich mit $\mathrm{NiMH}$ - und NiCD-Batterien höhere Wirkungsgrade erzielen lassen. Die coulombsche Effizienz von Li-lon-Batterien liegt typischerweise über 99 Prozent. Die coulombsche Effizienz bezeichnet die physikalisch mögliche Effizienz der Ladungsspeicherung und Ladungsfreigabe. Unter realen Bedingungen kommen noch ohmsche Verluste beim Lade- und Entladeprozess dazu, abhängig vom inneren Widerstand der Batterie und des verwendeten Stromflusses. Diese Verluste führen zu einer Aufwärmung der Batterie, was die Lebensdauer herabsetzen kann. Moderne Li-Ionenbatterien haben sehr geringe innere Widerstände, wodurch diese Wärmeverluste niedrig ausfallen. Trotzdem haben einige heutige Batteriesysteme eine Klimatisierung vorgesehen, welche die Batterietemperatur im Interesse einer verlängerten Lebensdauer kontrolliert. Dieses Thermomanagement macht bei der heutigen Fahrzeuggeneration den Hauptteil der Verluste aus, der Gesamtwirkungsgrad der Batterie kann je nach Temperatur zwischen 85 und 96 Prozent schwanken mit einem Mittelwert von 92 Prozent (Geringer 2012). Für diese Studie wurde ein Batteriegesamtwirkungsgrad von gegenwärtig 92 Prozent und eine langfristige Verbesserung (optimiertes Thermomanagement) auf 96 Prozent angenommen. Die Effizienz des Ladegerätes ist dabei nicht inbegriffen und wird separat berücksichtigt.

Lebensdauer: Ziel der Fahrzeugindustrie ist es, unter Normalbedingungen das Auswechseln der Batterien zu vermeiden. Konkret sollte eine Batterie mehr als 150000 Kilometer Fahrleistung überstehen und ein Alter von 12 Jahren erreichen. Aus Kundenperspektive sind lange Garantielaufzeiten für die Batterien wichtig. Für das PHEV Opel Ampera gilt z. B. eine Garantie von acht Jahren oder 160000 Kilometern, während Nissan und Mitsubishi fünf Jahre oder 100000 Kilometer Garantie für die Modelle Leaf und iMiev gewähren. In dieser Studie haben wir deshalb für 2012 eine Batterielebensdauer von 100000 Kilometer und nach 2012 von mehr als 150000 Kilometer angenommen. Daraus resultieren 
1,5 Batterien für einen Fahrzeuglebenszyklus im Jahr 2012 und nur noch eine Batterie für die zukünftigen Jahre (Althaus und Gauch 2010).

\subsection{Elektromotoren}

Elektromotoren konvertieren elektrische Energie in mechanische. In BEV und HEV operieren Elektromotoren auch umgekehrt - als Generator - und wandeln beim Bremsen mechanische Energie in Elektrizität zurück (Rekuperation).

\subsubsection{Gegenwärtiger Stand der Technologie}

\section{Bauformen}

Unterschiedliche Bauformen von Elektromotoren werden in HEV und BEV eingesetzt; bürstenlose Gleichstrommotoren, aber auch synchrone und asynchrone Wechselstrommotoren.

Synchronmotoren sind Wechselstrommotoren, die mit einer konstanten Drehzahl rotieren, welche einem Bruchteil der Wechselstromfrequenz entspricht. Die Drehzahl von Asynchronmotoren verlangsamt sich dagegen mit zunehmendem Drehmoment und das Magnetfeld wird mit Spulen und nicht mit Permanentmagneten erzeugt.
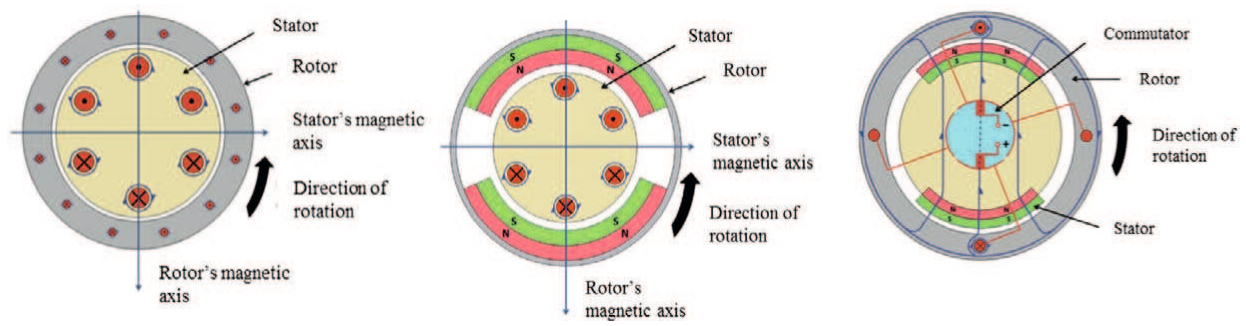

Abbildung 16: Schematische Darstellung eines Asynchronmotors (links), eines permanent magnetisierten Synchronmotors (Mitte) und eines permanent magnetisierten Gleichstrommotors (rechts). Adaptiert nach Pautzke 2010. 


\section{Effizienz}

Elektromotoren haben heute eine typische Effizienz von rund 90 Prozent. Bürstengleichstrommotoren sind besonders einfach zu steuern, weisen aber eine niedrigere Effizienz auf. Verbesserungen in der Regeltechnik haben die komplexe Steuerung von Wechselstrommotoren vereinfacht, weshalb einfache, aber ineffiziente Gleichstrommotoren an Bedeutung verlieren (Pautzke 2010).

Permanent magnetisierte Synchronmotoren weisen den höchsten Wirkungsgrad auf, da kein Induktionsstrom erzeugt werden muss. Die Herstellungskosten können jedoch aufgrund der teuren Permanentmagnete höher sein, auch wird für die leistungsfähigsten Magnete das Seltene-Erde-Element Neodym benötigt. Synchronmotoren mit Fremderregung sind günstiger und wegen der Induktionswicklung flexibler im Design. Die Vorteile von Asynchronmotoren liegen dagegen bei der einfachen und robusten Konstruktion. Sie benötigen kaum Unterhalt und werden daher häufig im Industriebereich eingesetzt (Neudorfer 2009). Der Nachteil der Asynchronmotoren liegt bei der zusätzlichen nötigen Induktionsspannung, was gegenüber permanent magnetisierten Synchronmotoren zu einer um etwa 2 Prozent niedrigeren Effizienz führt.

\subsubsection{Entwicklungspfade}

Jede Elektromotorbauform weist ihre Vor- und Nachteile auf. Je nach Anwendung sind daher unterschiedliche Bauformen optimal. Bei Elektroautos dominieren permanent magnetisierte Motoren. Gemäss einer EU-Marktanalyse der CE Delft (Duleep et al. 2011) werden in HEV und BEV gegenwärtig permanent magnetisierte, bürstenlose Motoren eingesetzt. Renault hingegen setzt bewusst auf Asynchronmotoren ohne Permanentmagnete. Gründe für Motoren mit $\mathrm{Pe}$ manentmagneten sind die hohe Energiedichte und die hohe energetische Effizienz. Der Entwicklungsstand der Technologie ist relativ weit fortgeschritten und in den letzten Jahren konnten deutliche Gewichtsreduktionen erzielt werden. Die Massenproduktion von Permanentmagneten hat zu Kostenreduktionen in der Motorenherstellung geführt (Neudorfer 2009) und die Entwicklung neuer hochenergetischer Permanentmagnetmaterialien und neuer amorpher Eisenlegierungen hat zu weiteren Effizienzsteigerungen geführt (Chau, Chan et al. 2008; Wang, Li et al. 2011). Obwohl sich die Technologie noch weiter verbessern kann, sind in den nächsten Jahrzehnten keine weiteren Technologiesprünge mehr zu erwarten. 


\section{Technologieentwicklung von Elektromotoren}

Die Effizienz von Elektromotoren ist weniger drehzahlabhängig als bei ICE-Motoren. Die Diagramme der Motorencharakteristik zeigen über einen weiten Einsatzbereich Wirkungsgrade von über 80 Prozent (Abbildung 17). Dennoch wurde für diese Studie zwischen den Einsatzbereichen innerorts und ausserorts unterschieden. Dadurch konnte berücksichtigt werden, dass Elektromotoren bei sehr niedrigen Geschwindigkeiten ebenfalls einen niedrigeren Wirkungsgrad aufweisen.

Hierzu wurden zwei unterschiedliche Elektromotortypen analysiert. Als Erstes wurden Motorcharakteristik-Diagramme eines Induktionsmotors und eines permanent magnetisierten Motors basierend auf den realen Motoreneigenschaften abgebildet (Abbildung 17).
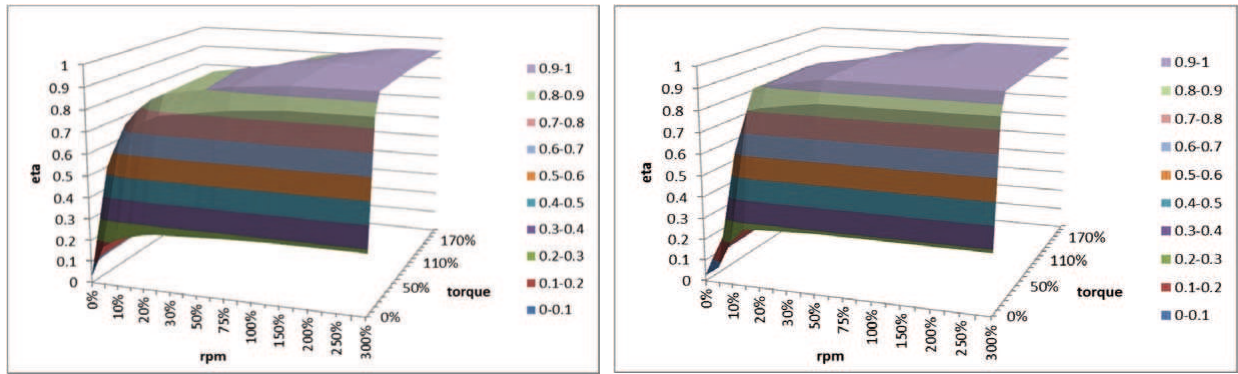

Abbildung 17: Eigenschaftsdiagramm eines asynchronen Induktionsmotors (links, Brusa ASM8.24.10) und eines permanent magnetisierten Hybridmotors (rechts, Brusa HSM6.17.12).

Dann wurden die detaillierten Wirkungsgrade für das Drehmomentverhalten im NEFZ-Zyklus berechnet. Daraus wurden schliesslich die durchschnittlichen Wirkungsgrade im Inner- und Ausserorts-NEFZ-Fahrzyklus abgeleitet (Abbildung 18). 

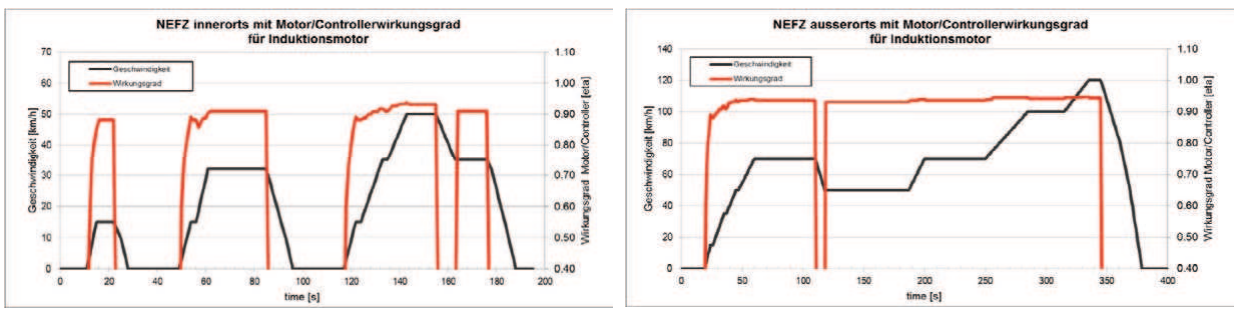

\begin{tabular}{|c|c|c|c|c|c|}
\hline $\begin{array}{r}\text { Energiebedarf } \\
\text { NEFZ } \\
\text { innerorts }\end{array}$ & $\begin{array}{r}\text { Wirkungsgrad } \\
\text { Durchschnitt } \\
\text { innerorts }\end{array}$ & $\begin{array}{r}\text { Energiebedarf } \\
\text { NEFZ } \\
\text { ausserorts }\end{array}$ & $\begin{array}{r}\text { Wirkungsgrad } \\
\text { Durchschnitt } \\
\text { ausserorts }\end{array}$ & $\begin{array}{r}\text { Energiebedarf } \\
\text { NEFZ } \\
\text { total }\end{array}$ & $\begin{array}{r}\text { Wirkungsgrad } \\
\text { Durchschnitt } \\
\text { total }\end{array}$ \\
\hline $\mathrm{kWh} / 100 \mathrm{~km}$ & $\%$ & $\mathrm{kWh} / 100 \mathrm{~km}$ & $\%$ & $\mathrm{kWh} / 100 \mathrm{~km}$ & $\%$ \\
\hline 13.31 & $89.4 \%$ & 14.84 & $93.8 \%$ & 14.28 & $92.3 \%$ \\
\hline Energiebedarf & Wirkungsgrad & Energiebedarf & Wirkungsgrad & Energiebedarf & Wirkungsgrad \\
\hline NEFZ & Durchschnitt & NEFZ & Durchschnitt & NEFZ & Durchschnitt \\
\hline innerorts & innerorts & ausserorts & ausserorts & total & \\
\hline $\mathrm{kWh} / 100 \mathrm{~km}$ & $\%$ & $\mathrm{kWh} / 100 \mathrm{~km}$ & $\%$ & $\mathrm{kWh} / 100 \mathrm{~km}$ & $\%$ \\
\hline 13.00 & $91.5 \%$ & 14.63 & $95.2 \%$ & 14.03 & $93.9 \%$ \\
\hline
\end{tabular}

Abbildung 18: Effizienzen eines asynchronen Induktionsmotors (Brusa ASM8.24.10, Abb. links, Tabelle oben) bei Betrieb innerorts und ausserorts. Vergleich mit permanent magnetisiertem Motor (Brusa HSM6.17.12, Abb. rechts, Tabelle unten), beide Motoren mit den gleichen Leistungswerten. Beispiel gerechnet für Auto der Kompaktklasse mit 1400 Kilogramm, verwendete Fahrzeugparameter siehe Kapitel 4.

Der untersuchte asynchrone Induktionsmotor ist eine Standardkonstruktion. Der permanent magnetisierte Motor ist eine fortschrittlichere und teurere Konstruktion, die für Elektrofahrzeuge optimiert ist und eine hohe Effizienz aufweist.

\subsubsection{Annahmen der Studie für die Elektromotor-Entwicklung}

Masse: Für 2012 wurde eine Motormasse inklusive Getriebe und Differenzial mit 100 Kilogramm für eine Leistung von 100 Kilowatt angenommen. Ein Topprodukt der Firma Brusa (HSM6.17.12 inkl. Getriebe/Differenzial) wiegt bereits heute nur 81 Kilogramm. Daraus leiten wir für die Entwicklung des Motorgewichts eine Reduktion um 10 Prozent pro betrachtetes Zeitintervall ab. Dies führt zu einem angenommenen Motorengewicht im Jahr 2035 von 81 Kilogramm. 
Wirkungsgrad: Für diese Studie wurde ein Wirkungsgrad von 91 Prozent für die Kombination von Motor und Kontroller angenommen, davon werden 2 Prozent Verluste für die Kraftübertragung abgezogen. Die resultierenden 89 Prozent sind repräsentativ für einen Motor der ersten Generation von Elektrofahrzeugen, der primär kostenoptimiert und nicht wirkungsgradoptimiert ist. Für den innerstädtischen Betrieb mit häufigem Start/Stopp wurde ein um 5 Prozent niedrigerer Wirkungsgrad angenommen. Für die Zukunft wurde ein Wirkungsgradgewinn (innerorts und ausserorts) von +1 Prozent bis 2020 , weitere +1 Prozent bis 2035 und weitere +1 Prozent bis 2050 angenommen. Somit erhält man 92 Prozent Wirkungsgrad für 2050 im extraurbanen Betrieb. Vergleich: Für einen heutigen Brusa-Motor HSM6.17.12 resultieren bereits heute 91 bzw. 95 Prozent im urbanen/ extraurbanen Betrieb, inklusive 2 Prozent Verlust für die Kraftübertragung sind es 89 bzw. 93 Prozent.

\begin{tabular}{|c|c|c|c|c|c|c|}
\hline Faktor & Einheit & & 2012 & 2020 & 2035 & 2050 \\
\hline \multirow{3}{*}{$\begin{array}{l}\text { Masse e-Drivetrain } \\
\text { Motor, Getriebe, } \\
\text { Controller }\end{array}$} & \multirow[t]{3}{*}{$\mathrm{kg}$} & ICE/HEV & - & - & - & - \\
\hline & & PHEV & 100 & 90 & 81 & 73 \\
\hline & & BEV & 100 & 90 & 81 & 73 \\
\hline \multirow{3}{*}{$\begin{array}{l}\text { eMotor Wirkungsgrad } \\
\text { NEFZ urban }\end{array}$} & \multirow[t]{3}{*}{$\%$} & ICE/HEV & $0 \%$ & $0 \%$ & $0 \%$ & $0 \%$ \\
\hline & & PHEV & $84 \%$ & $85 \%$ & $86 \%$ & $87 \%$ \\
\hline & & BEV & $84 \%$ & $85 \%$ & $86 \%$ & $87 \%$ \\
\hline \multirow{3}{*}{$\begin{array}{l}\text { eMotor Wirkungsgrad } \\
\text { NEFZ extraurban }\end{array}$} & \multirow[t]{3}{*}{$\%$} & ICE/HEV & $0 \%$ & $0 \%$ & $0 \%$ & $0 \%$ \\
\hline & & PHEV & $89 \%$ & $90 \%$ & $91 \%$ & $92 \%$ \\
\hline & & BEV & $89 \%$ & $90 \%$ & $91 \%$ & $92 \%$ \\
\hline
\end{tabular}

Tabelle 12: $\quad$ Annahmen für die Effizienz von Elektromotoren (innerorts/ ausserorts).

\subsection{Verbrennungsmotoren (ICE-Motoren)}

Obwohl diese Studie von Elektrofahrzeugen handelt, werden Vergleiche mit der Entwicklung der ICE-Fahrzeuge gemacht. Eine analoge Analyse des technologischen Fortschritts bei den ICE-Fahrzeugen ist daher sehr wichtig, um das Potenzial der Elektrofahrzeuge beurteilen zu können. Die Studie betrachtet durchschnittliche Werte für die gesamte Bandbreite von ICE-Motoren ohne Feinunterscheidung individueller Motorenkonzepte (z.B. Benzin- oder Dieselmotor mit und ohne Aufladung etc.). 


\subsubsection{Gegenwärtiger Stand der Technologie}

\section{Effizienzannahmen für die Studie}

ICE-Motoren weisen komplexe Eigenschaften mit einem relativ engen Optimum auf. Der beste Wirkungsgrad wird bei hoher Last und mittlerer Drehzahl erreicht. Meist wird aber in Bereichen ausserhalb des optimalen Betriebspunkts gefahren. Analog zu den Annahmen bei den Elektromotoren wurde in dieser Studie auch bei den ICE-Motoren zwischen einem durchschnittlichen Wirkungsgrad innerorts (geringe Lasten, variable Drehzahlen, relativ niedriger Wirkungsgrad) und ausserorts (höhere Lasten, konstantere Drehzahlen, höherer Wirkungsgrad) unterschieden.

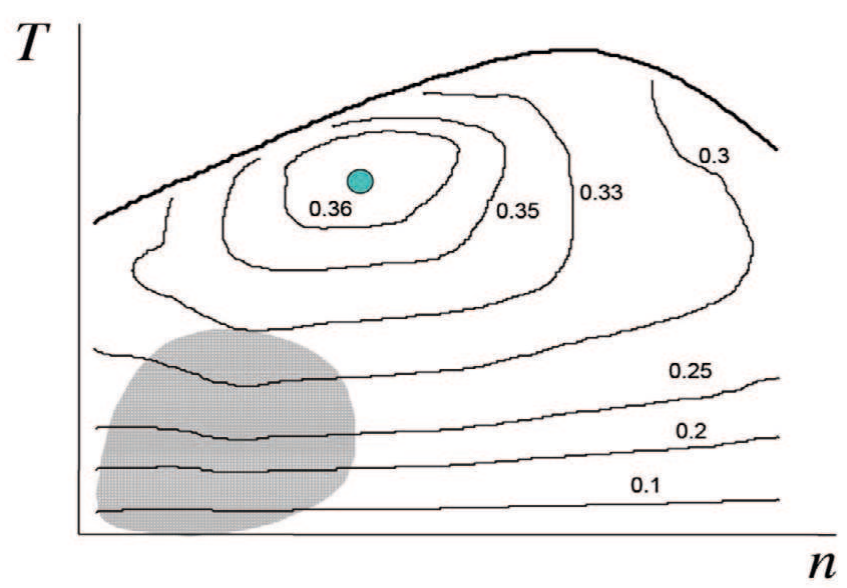

Abbildung 19: Last-Drehzahl-Diagramm (T-n) mit den charakteristischen Wirkungsgraden eines ICE-Motors: Der typische Einsatzbereich (graues Feld) liegt ausserhalb des optimalen Betriebspunkts (grauer Punkt), (Diagramm: ETHZ).

\subsubsection{Entwicklungspfade}

Eine Vielzahl an Massnahmen können einzeln oder in Kombination zur Verbesserung der Effizienz der ICE-Motoren eingesetzt werden: Downsizing (kleinerer Motor), Turboaufladung oder mechanische Kompression, direkte Kraftstoffein- 
spritzung mit höherem Druck, optimierte Zylinderkopfgeometrie, Verringerung der inneren Reibung und Zylinderabschaltung. Getriebe mit mehr Gängen ermöglichen den Betrieb näher am Punkt des optimalen Wirkungsgrades (siehe Abbildung 19). Bremsenergierückgewinnung, Start-Stopp-Automatik, Elektrifizierung der mechanisch angetriebenen Komponenten sind Massnahmen, die bereits heute Stand der Technik für die Oberklassefahrzeuge sind, und die Abwärmenutzung durch thermoelektrische Geräte befindet sich in Entwicklung. All diese Massnahmen tragen wesentlich zur Verbesserung der Effizienz bei, das Verbesserungspotenzial für die Umwandlung von Brennstoffenergie in Bewegungsenergie ist jedoch bezüglich Betriebstemperaturen und Materialeigenschaften begrenzt. Generell ist aber das energetische Verbesserungspotenzial bei ICE-Motoren immer noch überraschend hoch, da die Verbrauchsoptimierung erst seit etwa zehn Jahren ein Entwicklungstreiber ist. Vorher stand eher die Leistungs- und Abgasoptimierung im Vordergrund.

Die besten heute erhältlichen Technologien mit Benzinverbrennungsmotoren weisen Stadt/Überland/Autobahn-Wirkungsgrade von respektive 21, 27 oder 30 Prozent auf (Beispiel VW Touran 1.4 TSi DSG6 (Bach 2012)). Bei fortschrittlichen Vollhybridfahrzeugen wie dem Toyota Prius II lässt sich besonders im urbanen Einsatz der Wirkungsgrad steigern auf 29 bzw. 32 und 33 Prozent.

Ein zukunftsweisendes Konzept zur Erzielung eines noch tieferen $\mathrm{CO}_{2}$-Ausstosses ist die Kombination eines Erdgasmotors mit Hybridisierung, mit welchem ähnlich gute Wirkungsgrade wie bei guten Benzinhybriden erzielt werden können, und welches zusätzlich den Vorteil des prinzipiell um rund 20 Prozent tieferen $\mathrm{CO}_{2}$-Gehaltes von Erdgas im Vergleich mit Benzin bei gleicher Energiemenge aufweist (Beispiel: Erdgas-Hybridfahrzeug CLEVER der Empa (Bach 2008)). Damit lassen sich heute schon die Zielwerte der EU für den $\mathrm{CO}_{2}$-Ausstoss eines Fahrzeugs im Jahr 2020 unterbieten ( $\left.<95 \mathrm{~g} \mathrm{CO}_{2} / \mathrm{km}\right)$.

\subsubsection{Annahmen der Studie für die ICE-Motorenentwicklung}

Für die heutigen durchschnittlichen Wirkungsgrade wurden 17 Prozent im innerstädtischen Verkehr (ECE-Verbrauch bei Euro-4-Fahrzeugen Modelljahr 2004 bis 2006) und 27 Prozent ausserorts angenommen (Bach 2012). Für Inner- und Ausserortsbetrieb wird ein Wirkungsgradzuwachs von +3 Prozent resp. +2 Prozent bis 2020 angenommen, zusätzliche +3 bzw. +2 Prozent bis 2035 und nochmals +3 bzw. +2 Prozent bis 2050 . Diese Verbesserungen werden durch die 
kontinuierliche Einführung der oben aufgezählten Massnahmen erreicht. Eine grössere Wirkungsgradverbesserung wird für den urbanen Betrieb angenommen, weil davon ausgegangen wird, dass ab 2020 milde Hybridisierung mit der Möglichkeit der teilweisen Rekuperation von Bremsenergie als Standard gelten wird (Tabelle 13). Diese Werte werden für die durchschnittliche Neuwagenflotte im jeweiligen Verkaufsjahr angenommen.

\begin{tabular}{|c|c|c|c|c|c|c|}
\hline Faktor & Einheit & & 2012 & 2020 & 2035 & 2050 \\
\hline \multirow{3}{*}{$\begin{array}{l}\text { Masse ICE Drivetrain } \\
\text { Motor, Getriebe, } \\
\text { Treibstoff, Abgas etc. }\end{array}$} & \multirow[t]{3}{*}{$\mathrm{kg}$} & ICE/HEV & 150 & 135 & 122 & 109 \\
\hline & & PHEV & 100 & 90 & 81 & 73 \\
\hline & & BEV & - & - & - & - \\
\hline \multirow{3}{*}{$\begin{array}{l}\text { ICE-Wirkungsgrad } \\
\text { NEFZ urban }\end{array}$} & \multirow[t]{3}{*}{$\%$} & ICE/HEV & $17 \%$ & $20 \%$ & $23 \%$ & $26 \%$ \\
\hline & & PHEV & $25 \%$ & $28 \%$ & $31 \%$ & $34 \%$ \\
\hline & & BEV & $0 \%$ & $0 \%$ & $0 \%$ & $0 \%$ \\
\hline \multirow{3}{*}{$\begin{array}{l}\text { ICE-Wirkungsgrad } \\
\text { NEFZ extraurban }\end{array}$} & \multirow[t]{3}{*}{$\%$} & ICE/HEV & $27 \%$ & $29 \%$ & $31 \%$ & $33 \%$ \\
\hline & & PHEV & $28 \%$ & $30 \%$ & $32 \%$ & $34 \%$ \\
\hline & & BEV & $0 \%$ & $0 \%$ & $0 \%$ & $0 \%$ \\
\hline
\end{tabular}

Tabelle 13: $\quad$ Angenommene Motorenwirkungsgrade und Verbrauch für Fahrzeuge der Kompaktklasse im NEFZ-Verbrauchszyklus und im realen Betrieb.

Zur Plausibilisierung der Annahmen kann ein Vergleich mit den politisch definierten Reduktionszielen gemacht werden (Abbildung 20). Diese $\mathrm{CO}_{2}$-Reduktionsziele gelten als ambitiös, aber technisch machbar, wenn alle Verbesserungsmöglichkeiten kombiniert eingesetzt werden.

Abbildung 21 zeigt den Vergleich der in dieser Studie angenommenen technologischen Entwicklung mit den EU-Zielwerten. Die Entwicklung nach 2025 wurde von den Autoren angenommen und sollte als grober Richtwert betrachtet werden. 


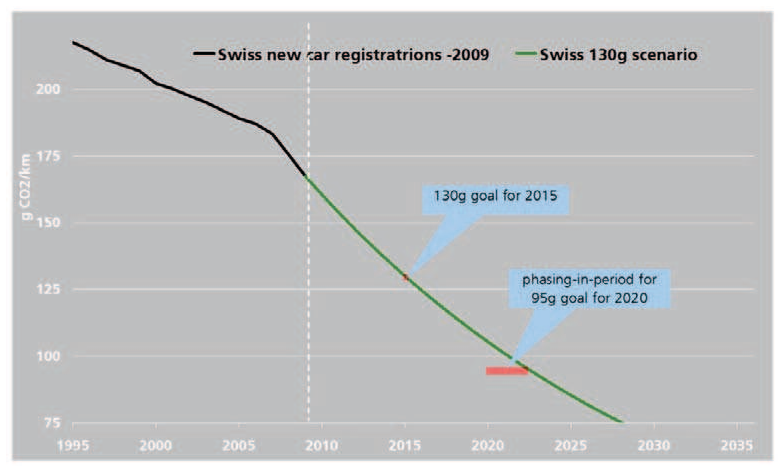

Abbildung 20: Entwicklung der $\mathrm{CO}_{2}$-Emissionen der $\mathrm{CH}$-Autoverkäufe im NEFZ-Fahrzyklus seit 1995 sowie Zielwerte der $\mathrm{CO}_{2}$-Emissionen (130 g/km im Jahr 2015, $95 \mathrm{~g} / \mathrm{km}$ im Jahr 2020 (EU-Ziel) bzw. 2023 (angepasstes $\mathrm{CH}$-Szenario).

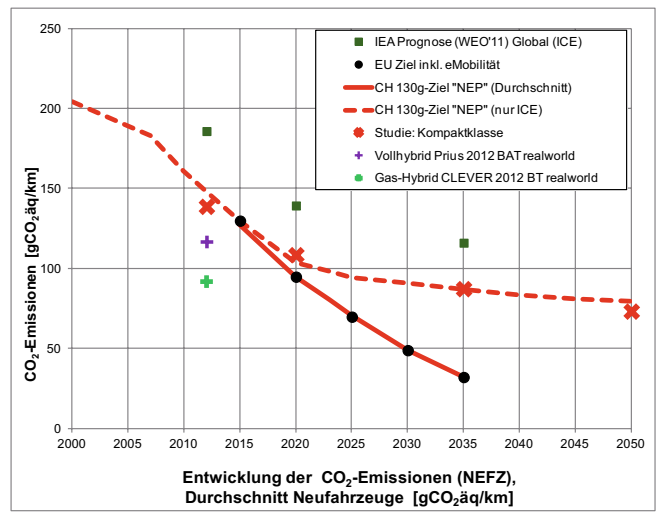

Abbildung 21: Zielwert der EU für $\mathrm{CO}_{2}$-Emissionen von Neufahrzeugen (schwarze Punkte) und für die Schweiz (rote Linie) inklusive Elektrofahrzeuge, die gemäss heutigem Usus mit $0 \mathrm{~g} \mathrm{CO}_{2} / \mathrm{km}$ eingesetzt werden. Die roten Kreuze bezeichnen die ICE-Fahrzeugentwicklung der Kompaktklasse in der Studie. Als Vergleich wird die Prognose für ICE-Fahrzeuge der IEA gezeigt (schwarze Quadrate). Zur Orientierung über das technische Potenzial dienen die Pluszeichen (+), blau für den Toyota Prius Vollhybrid im realen Betrieb und grün für das Erdgas-Hybridfahrzeug CLEVER der Empa im realen Betrieb. 


\section{Technologieentwicklung für PHEV}

Für heutige serielle PHEV (EV mit Range Extender) wurden durchschnittliche Wirkungsgrade von 27 Prozent im innerstädtischen Verkehr und 30 Prozent ausserorts angenommen. Sowohl für innerorts als auch für ausserorts wird ein Wirkungsgradgewinn von +3 Prozent bis 2020, zusätzliche +4 Prozent bis 2035 und nochmals +3 Prozent bis 2050 angenommen.

Für die Studie wird davon ausgegangen, dass die elektrische Auflademöglichkeit der PHEV-Fahrzeuge tatsächlich genutzt wird, das heisst, dass die ersten Kilometer rein elektrisch gefahren werden (Plug-In-Betriebsanteil), bevor der Range Extender eingesetzt wird. Bei laufendem Verbrennungsmotor im Range-Extender-Betrieb verbrauchen PHEV-Fahrzeuge allerdings ähnlich viel wie reine ICEFahrzeuge. Im Lauf der Entwicklung steigt der Anteil elektrisch gefahrener Kilometer an (bedingt durch höhere Batteriekapazitäten) und der Benzinverbrauch des Range Extenders nimmt entsprechend ab. Die durchschnittlich gefahrene Tagesdistanz in der Schweiz beträgt weniger als 40 Kilometer (BFS 2012), die ersten verfügbaren Plug-In-Fahrzeuge (Opel Ampera bzw. Chevrolet Volt) haben eine elektrische Reichweite von rund 50 Kilometern.

\begin{tabular}{|c|c|c|c|c|c|c|}
\hline Faktor & Einheit & & 2012 & 2020 & 2035 & 2050 \\
\hline \multirow{3}{*}{$\begin{array}{l}\text { Wirkungsgrad NEFZ } \\
\text { urban }\end{array}$} & \multirow[t]{3}{*}{$\%$} & ICE/HEV & & & & \\
\hline & & PHEV & $25 \%$ & $28 \%$ & $31 \%$ & $34 \%$ \\
\hline & & BEV & & & & \\
\hline \multirow{3}{*}{$\begin{array}{l}\text { Wirkungsgrad NEFZ } \\
\text { extraurban }\end{array}$} & \multirow[t]{3}{*}{$\%$} & ICE/HEV & & & & \\
\hline & & PHEV & $28 \%$ & $30 \%$ & $32 \%$ & $34 \%$ \\
\hline & & BEV & & & & \\
\hline \multirow[t]{3}{*}{ Plug-In-Betriebsanteil } & \multirow[t]{3}{*}{$\%$} & ICE/HEV & & & & \\
\hline & & PHEV & $60 \%$ & $75 \%$ & $85 \%$ & $90 \%$ \\
\hline & & BEV & & & & \\
\hline
\end{tabular}

Tabelle 14: $\quad$ Angenommene Motorenwirkungsgrade und Verbrauch für den Range Extender im PHEV. 


\subsection{Ladegeräte}

Elektrofahrzeuge brauchen ein Ladesystem, um die Batterie aufzuladen. Hauptziel des Ladegeräts ist es, den Strom so rasch wie möglich in die Batterie zu «pumpen», unter Vermeidung von Überhitzung, Batteriedegradation und einer Überlastung des Netzanschlusses.

Mehr Stromfluss zum Auto verkürzt die Ladezeit. Einige Arten von Batterien, wie Lithium-Titanat, LiFePO4 und sogar bestimmte NiMH-Varianten, könnten innert 10 bis 20 Minuten praktisch vollständig geladen werden. Die Stromversorgung ist aber in der Praxis durch die Kapazität des Netzanschlusses und die Leistung der On-Board-Ladeelektronik begrenzt.

\subsubsection{Gegenwärtiger Stand der Technologie}

Ein normaler einphasiger Haushaltanschluss (230 V AC, Absicherung 10 oder 16 A) kann maximal etwa 3,5 Kilowatt liefern. Elektrofahrzeuge haben normalerweise eingebaute Ladegeräte, mit denen einphasiger (230 V) oder dreiphasiger (400 V) Wechselstrom auf die Gleichspannung geregelt wird, die zur Ladung der Batterie notwendig ist (Boulanger, Chu et al. 2011). Die Ladeleistung ist bei einigen Fahrzeugen auf 3,3 Kilowatt begrenzt, um die On-Board-Hardwarekosten zu senken. Andere Fahrzeuge ertragen deutlich höhere Ströme. Der Tesla Roadster lässt sich beispielsweise mit 16,8 Kilowatt $(230 \mathrm{~V}$ bei $70 \mathrm{~A})$ über den «Tesla Home Connector» laden. Elektroautos, die den CHAdeMO-Standard unterstützen, lassen sich mit 50 Kilowatt schnellladen.

Ein 3,3 Kilowatt-Ladegerät kann ein durchschnittliches BEV in 6 bis 8 Stunden laden. 10-Kilowatt-Ladegeräte reduzieren die Ladezeit auf 2 bis 3 Stunden. Schnellladen mit 50 Kilowatt ermöglicht Ladezeiten von etwa 30 Minuten. In Entwicklung befinden sich Gleichstrom-Direktladungssysteme mit 100 Kilowatt (NPE 2011). Damit würde sich die Ladezeit auf 15 Minuten reduzieren. Leistungsfähige Schnellladesysteme haben jedoch den Nachteil grösserer notwendiger Infrastrukturanpassungen und damit deutlich höherer Kosten. Weiter können hohe Ladeströme die Lebensdauer der Batterien markant verkürzen.

Im Vergleich zur Betankung mit Treibstoff sind auch bei Schnellladung die Ladezeiten immer noch sehr lang, und falls Fahrzeuge mit grösseren Stromspeichern geladen werden müssen (Fullsize-PKW, Busse), verlängern sich die Ladezeiten 
entsprechend. Der Vorteil der elektrischen Betankung liegt dafür bei der Möglichkeit, zu Hause oder am Arbeitsplatz unabhängig von einer öffentlichen Tankstelleninfrastruktur laden zu können. Voraussetzung dazu ist der Zugang zu einer Steckdose, was jedoch in Tiefgaragen oder bei Aussenparkplätzen bisher nicht immer gegeben ist.

\subsubsection{Entwicklungspfade}

Die Herausforderung bei der zukünftigen Entwicklung liegt nicht so sehr bei technischen Hürden, sondern vielmehr bei der Etablierung einheitlicher Standards für Lademethoden und Stecker. Gegenwärtig befinden sich unterschiedliche Standards in paralleler Entwicklung, was für die Akzeptanz und Penetration der Elektromobilität hinderlich ist.

Vielversprechende Optionen werden im Bereich kabelloses Laden (induktive Ladesysteme) diskutiert. Diese befinden sich jedoch noch in der Entwicklungsphase und werden erst in Pilotprojekten eingesetzt. Im Rahmen dieses Berichts wird deshalb nicht weiter darauf eingegangen.

\section{Standardisierung der Lademethode}

In der Schweiz und in Europa hat sich der europäische Autoherstellerverband (Association des Constructeurs Européens d'Automobiles, ACEA) für den IEC 62196 der International Electrotechnical Commission (IEC) entschieden. In Nordamerika gilt dagegen der SAE- J1772-Standard der Society of Automotive Engineers (SAE).

\section{Europäischer Standard IEC 62196}

Die europäische IEC-62196-Norm kennt vier Lademodi. Modi 1 und 2 sind für langsames Laden ab Haushaltanschluss vorgesehen. Modus 3 definiert Wechselstromladen ab spezifischen, hoch abgesicherten Ladestationen und Modus 4 definiert Gleichspannungs-Schnellladen mittels externem Ladegerät direkt auf die Batterie (siehe Tabelle 15).

\section{Design des Ladesteckers}

Im Jahr 2011 hat die IEC die technischen Spezifikationen für die Basisvarianten von Ladesteckern finalisiert. Der ideale Ladestecker sollte Elektromobilität in al- 
len Teilen der Welt ermöglichen. Der IEC-62196-Standard definiert vier Designtypen. Der Typ-2-Stecker soll mit allen relevanten Leistungsniveaus zwischen einphasigen AC-Hausanschlüssen bis zu leistungsfähigen dreiphasigen 63-AAnschlüssen umgehen können. Zusätzlich ist er für zukünftige DC-Ladestationen bis 30 Kilowatt geeignet. Typ-2-Stecker wurden deshalb als Standardvorschlag für Europa definiert (NPE 2011) und unterstützen Ladeströme von 16, 32 und 63 Ampère mit Leistungen von 3,3 bis 43,5 Kilowattstunden abhängig von der Ladespannung.

\begin{tabular}{llll} 
Method & Voltage & Max current & Charger \\
\hline Mode 1 & 250 VAC, 1-phase; & 16 A & on-board \\
& 480 VAC, 3-phase & & \\
Mode 2 & $\begin{array}{l}\text { 250 VAC, 1-phase; } \\
\text { 480 VAC, 3-phase }\end{array}$ & $32 \mathrm{~A}$ & on-board \\
Mode 3 & $\begin{array}{l}\text { 250 VAC, 1-phase; } \\
\text { 480 VAC, 3-phase }\end{array}$ & $250 \mathrm{~A}$ & on-board \\
Mode 4 & 300-600 VDC & $400 \mathrm{~A}$ & off-board
\end{tabular}

Tabelle 15: $\quad$ Europäische Ladenormen.

\section{Design des Fahrzeuganschlusses}

Auf der Fahrzeugseite sind Entwicklungen zur Definition eines standardisierten Anschlusses für verschiedene Ladesysteme in Gang. Das universale «EV Combined Charging System» akzeptiert vier verschiedene Ladeoptionen von einphasiger Wechselspannung bis Gleichstromschnellladung mit nur einem Anschluss auf der Fahrzeugseite. Wichtige globale Automobilfirmen aus den USA und aus Deutschland unterstützen diese Option.

\section{Verbesserung des Lademanagements}

Elektrofahrzeuge können prinzipiell an jeder Steckdose zu jeder Zeit geladen werden. Zunehmend werden Lösungen implementiert, mit denen der Stromfluss unidirektional (Grid-To-Vehicle) oder bidirektional (Vehicle-To-Grid) gezielt kontrolliert werden kann. Beispielsweise kann das Fahrzeug zur Kosteneinsparung so programmiert werden, dass es nur während Niedrigtarifzeiten geladen wird. 
Wünschenswert im Sinne der Stromnetzbetreiber wäre die Ladung nur zu Zeiten, in denen ein Überschuss an Bandenergie im Stromnetz vorhanden ist. VehicleTo-Grid-Ideen gehen dahin, dass aus den Fahrzeugen zu Strombedarfspeakzeiten Energie aus den Batterien ins Stromnetz zurückgespiesen werden könnte.

\subsubsection{Annahmen der Studie für die Entwicklung der Ladegeräte}

\begin{tabular}{|c|c|c|c|c|c|c|}
\hline Faktor & Einheit & & 2012 & 2020 & 2035 & 2050 \\
\hline \multirow{3}{*}{$\begin{array}{l}\text { Wirkungsgrad } \\
\text { Ladegerät } \\
\text { durchschnittlich }\end{array}$} & \multirow[t]{3}{*}{$\%$} & ICE/HEV & $0 \%$ & $0 \%$ & $0 \%$ & $0 \%$ \\
\hline & & PHEV & $90 \%$ & $93 \%$ & $94 \%$ & $95 \%$ \\
\hline & & BEV & $90 \%$ & $93 \%$ & $94 \%$ & $95 \%$ \\
\hline
\end{tabular}

Tabelle 16: $\quad$ Annahmen für die Effizienz von Ladegeräten.

Heutiger Startpunkt für die Effizienz von Batterieladegeräten ist 90 Prozent, basierend auf den Untersuchungen verschiedener aktueller Elektrofahrzeuge (Geringer 2012). Einige Geräte erreichen bereits höhere Effizienzen, wie die NLGSerie der Firma Brusa mit 96 Prozent typischer Effizienz. Kleinere, technisch einfache Ladegeräte für Zweiräder können sehr tiefe Wirkungsgrade um 80 Prozent aufweisen. Ähnlich wie bei der Entwicklung von Motorencontrollern und in Anlehnung an die Erfahrungen mit Geräten aus der Informations- und Kommunikationstechnologie wird für die kommenden Jahre eine Technologieentwicklung in Richtung kostengünstigerer und fortschrittlicherer Elektronik erwartet.

\subsection{Sicherheit und Komfort}

Die hohen Ansprüche an Komfort im Auto haben zu einem hohen Entwicklungsstand geführt. Heute verfügen auch Autos der kleinen Klassen über Ausstattungen, die früher den Oberklassefahrzeugen vorbehalten waren.

\subsubsection{Gegenwärtiger Stand der Technologie}

\section{Sicherheit und Unterhaltung}

Eine Vielzahl von elektrischen Verbrauchern dient zur Sicherheit und Unterhaltung. Dazu zählen zum Beispiel Beleuchtung, Scheibenwischer, Soundsystem, 
Navigation, Abstandswarner und Rückfahrkameras. Alle diese Geräte werden mit Strom betrieben, der bei ICE-Antrieben über einen Alternator erzeugt werden muss und bei Elektrofahrzeugen direkt der Batterie entnommen wird.

\section{Heizung}

Die meisten heutigen Elektrofahrzeuge nutzen ein sehr einfaches und günstiges, dafür äusserst ineffizientes Heizverfahren, nämlich die elektrische Widerstandsheizung. Der thermische Energiebedarf eines Personenwagens ist ähnlich hoch wie der eines kleines Einfamilienhauses, da die Fahrzeuge nicht isoliert sind. Dies ergibt einen sehr hohen Heizenergiebedarf, der über die Batterie gedeckt werden muss, was die Reichweite von BEV massiv reduziert. ICE-Fahrzeuge benötigen keine zusätzliche Heizenergie, sondern beziehen diese aus der Abwärme des Verbrennungsmotors. Diese «Gratisenergie» ist der Hauptgrund (neben dem Gewicht), weshalb Wärmeisolation bei Autos noch nie thematisiert wurde.

\section{Kühlung}

Fahrzeugkühlung erfolgt mittels Klimaanlagen, welche auf Wärmepumpen beruhen. Die Effizienz einer Wärmepumpe wird mit dem Wirkungsgrad COP (Coefficient Of Performance) beschrieben.

\subsubsection{Entwicklungspfad}

Gegenwärtig werden bidirektionale Wärmepumpen entwickelt, die sowohl zum Kühlen als auch zum Heizen eingesetzt werden können. Dadurch lässt sich der Wärmeenergiebedarf deutlich senken, was wiederum zu höheren elektrischen Reichweiten führt. Der Renault ZOE (angekündigt auf Frühjahr 2013) ist das erste Serienfahrzeug, welches eine solche bi-direktionale Wärmepumpe aufweist.

Für diese Studie wurde angenommen, dass die nächste Generation elektrischer Fahrzeuge ab 2020 mit bidirektionalen Wärmepumpen gekühlt und beheizt wird. Obwohl thermische Isolation (z.B. Doppelverglasung) energetische Vorteile für Kühlung und Heizen bringen würde, wird Isolation nicht in dieser Studie berücksichtigt, da die zukünftige Marktpenetration aufgrund der Komplexität unsicher ist. 


\title{
3.6.3 Annahmen der Studie für die Entwicklung von Sicherheit und Komfort
}

Der Bedarf für Sicherheit und Komfort wird über die Jahre als konstant angenommen.

\begin{abstract}
Scheinwerfer, Scheibenwischer und andere elektrische Verbraucher (Lüftung, Unterhaltung, Navigation etc.): Der kombinierte Stromverbrauch von $1 \mathrm{kWh} / 100 \mathrm{~km}$ wird als konstant für die Fahrzeugklassen Fullsize, Compact und Micro angenommen für die Periode 2010 bis 2050 (Althaus und Gauch 2010). Dies entspricht 500 Watt Leistung bei konstantem Gebrauch bei einer Durchschnittsgeschwindigkeit von 50 Stundenkilometern. Für Kleinst-EV (3-Wheeler) wurde ein Bedarf von 0,6 kWh/100 km angenommen. In jedem Fall wird der Strombedarf aus der Batterie gedeckt.
\end{abstract}

Heizung: Für die Periode 2010 bis 2050 wird ein konstanter durchschnittlicher thermischer Energiebedarf von 3 kWh/100 km angenommen (Althaus und Gauch 2010). Dies entspricht 3 Kilowatt durchschnittlicher Heizleistung während sechs Monaten im Jahr bei einer Durchschnittsgeschwindigkeit von $50 \mathrm{~km} / \mathrm{h}^{1}$. Heizsysteme von PHEV und BEV basieren heute (2012) auf Widerstandsheizungen. In dieser Studie wird angenommen, dass ab 2020 nur noch Wärmepumpen mit einer Leistungskennzahl (Coefficient Of performance, COP) von 2,0 und ab 2035 mit einer COP-Zahl von drei eingesetzt werden (Tabelle 17). Für ICE-Fahrzeuge wird angenommen, dass der Heizenergiebedarf vollständig mit Motorenabwärme gedeckt wird.

Klimatisierung: Es wird ein konstanter Energiebedarf von $1 \mathrm{kWh} / 100 \mathrm{~km}$ angenommen (Althaus und Gauch 2010). Dies entspricht einer Leistung von 1,5 Kilowatt während vier Monaten im Jahr bei einer Durchschnittsgeschwindigkeit von 
50 Stundenkilometern. Dies gilt für alle Fahrzeugkategorien und für die gesamte Periode von 2012 bis 2050. Als Wirkungsgrad wird für 2012 eine COP-Zahl von zwei angenommen, die sich bis auf drei verbessert (ab 2035).

Gemäss der Studie von Geringer und Tobler (2012) beträgt der Energiemehrverbrauch durch die Klimaanlage $3 \mathrm{kWh} / 100 \mathrm{~km}$, wobei die besten Fahrzeuge (Nissan Leaf, Mitsubishi iMiev) nur $1 \mathrm{kWh} / 100 \mathrm{~km}$ benötigen. In europäischen Klimaverhältnissen herrschen nur drei Monate pro Jahr Temperaturen über $20^{\circ} \mathrm{C}$, im Jahresschnitt würde dies nur 0,5 Kilowattstunden Durchschnittsenergiebedarf durch die Klimaanlage bedeuten ( $3 \mathrm{kWh} \times 2 / 12$ Monate). Allerdings werden heute automatisierte Klimaanlagen zur Vermeidung von beschlagenen Scheiben schon ab $5^{\circ} \mathrm{C}$ eingeschaltet (Weilenmann, Alvarez et al. 2010), weshalb die Studie von einem etwas höheren Wert ausgeht. Die neusten Klimaanlagen verfügen über Taumelscheibenkompressoren mit einem niedrigeren Energieverbrauch.

\begin{tabular}{|c|c|c|c|c|c|c|}
\hline Faktor & Einheit & & 2012 & 2020 & 2035 & 2050 \\
\hline \multirow{3}{*}{$\begin{array}{l}\text { Sicherheit und } \\
\text { Unterhaltung } \\
\text { Leistungsbedarf }\end{array}$} & \multirow{3}{*}{$\begin{array}{l}\text { kW } \\
\text { wäh- } \\
\text { rend } \\
\text { Zeit-\% }\end{array}$} & ICE/HEV & $0,5(100 \%)$ & $0,5(100 \%)$ & $0,5(100 \%)$ & $0,5(100 \%)$ \\
\hline & & PHEV & $0,5(100 \%)$ & $0,5(100 \%)$ & $0,5(100 \%)$ & $0,5(100 \%)$ \\
\hline & & BEV & $0,5(100 \%)$ & $0,5(100 \%)$ & $0,5(100 \%)$ & $0,5(100 \%)$ \\
\hline \multirow{3}{*}{$\begin{array}{l}\text { Sicherheit und } \\
\text { Unterhaltung } \\
\text { Verbrauch } \\
\text { @50km/h avg. }\end{array}$} & \multirow{3}{*}{$\begin{array}{l}\text { kWh/ } \\
100 \mathrm{~km}\end{array}$} & ICE/HEV & 1,0 & 1,0 & 1,0 & 1,0 \\
\hline & & PHEV & 1,0 & 1,0 & 1,0 & 1,0 \\
\hline & & BEV & 1,0 & 1,0 & 1,0 & 1,0 \\
\hline \multirow{3}{*}{$\begin{array}{l}\text { Heizung } \\
\text { Leistungsbedarf }\end{array}$} & \multirow{3}{*}{$\begin{array}{l}\text { kW } \\
\text { wäh- } \\
\text { rend } \\
\text { Zeit-\% }\end{array}$} & ICE/HEV & $3,0(50 \%)$ & $3,0(50 \%)$ & $3,0(50 \%)$ & $3,0(50 \%)$ \\
\hline & & PHEV & $3,0(50 \%)$ & $3,0(50 \%)$ & $3,0(50 \%)$ & $3,0(50 \%)$ \\
\hline & & BEV & $3,0(50 \%)$ & $3,0(50 \%)$ & $3,0(50 \%)$ & $3,0(50 \%)$ \\
\hline \multirow{3}{*}{$\begin{array}{l}\text { Heizung Verbrauch } \\
\text { @50km/h avg. }\end{array}$} & \multirow{3}{*}{$\begin{array}{l}\text { kWh/ } \\
100 \mathrm{~km}\end{array}$} & ICE/HEV & - & - & - & - \\
\hline & & PHEV & 3,0 & 1,5 & 1,0 & 1,0 \\
\hline & & BEV & 3,0 & 1,5 & 1,0 & 1,0 \\
\hline \multirow{3}{*}{$\begin{array}{l}\text { Kühlung } \\
\text { Leistungsbedarf }\end{array}$} & \multirow{3}{*}{$\begin{array}{l}\mathrm{kW} \\
\text { wäh- } \\
\text { rend } \\
\text { Zeit-\% }\end{array}$} & ICE/HEV & $1,5(33 \%)$ & $1,5(33 \%)$ & $1,5(33 \%)$ & $1,5(33 \%)$ \\
\hline & & PHEV & $1,5(33 \%)$ & $1,5(33 \%)$ & $1,5(33 \%)$ & $1,5(33 \%)$ \\
\hline & & BEV & $1,5(33 \%)$ & $1,5(33 \%)$ & $1,5(33 \%)$ & $1,5(33 \%)$ \\
\hline \multirow{3}{*}{$\begin{array}{l}\text { Kühlung Verbrauch } \\
\text { @ } 50 \mathrm{~km} / \mathrm{h} \\
\text { Durchschnitt }\end{array}$} & \multirow{3}{*}{$\begin{array}{l}\mathrm{kWh} / \\
100 \mathrm{~km}\end{array}$} & ICE/HEV & 0.5 & 0.4 & 0.3 & 0.3 \\
\hline & & PHEV & 0.5 & 0.4 & 0.3 & 0.3 \\
\hline & & BEV & 0.5 & 0.4 & 0.3 & 0.3 \\
\hline \multirow{3}{*}{$\begin{array}{l}\text { Total Sicherheit } \\
\text { und Komfort } \\
\text { Benzin/Strombedarf }\end{array}$} & \multirow{3}{*}{$\begin{array}{l}\mathrm{l} / 100 \mathrm{~km} \\
\mathrm{kWh} / \\
100 \mathrm{~km}\end{array}$} & ICE/HEV & $1.0 \mathrm{I}$ & $0.8 \mathrm{I}$ & 0.7 I & $0.6 I$ \\
\hline & & PHEV & $4.5 \mathrm{kWh}$ & $2.9 \mathrm{kWh}$ & $2.3 \mathrm{kWh}$ & $2.3 \mathrm{kWh}$ \\
\hline & & BEV & $4.5 \mathrm{kWh}$ & $2.9 \mathrm{kWh}$ & $2.3 \mathrm{kWh}$ & $2.3 \mathrm{kWh}$ \\
\hline
\end{tabular}

Tabelle 17: $\quad$ Annahmen für den Energiebedarf für Sicherheit und Komfort. 


\section{$4 \quad$ Zukünftige Entwicklung der Fahrzeuge}

\subsection{Untersuchungsrahmen}

\subsubsection{Untersuchte Fahrzeugtypen}

Die unterschiedlichsten Fahrzeugtypen mit sehr verschiedenen funktionellen und technischen Eigenschaften werden in der Schweiz verkauft. Um Aussagen über die Entwicklung im gesamten Individualverkehr machen zu können, muss aus einzelnen Fahrzeugtypen eine Gesamtflotte definiert werden. In Kapitel 2.6 wurde als Ausgangsbasis für die Mikrosimulation der Flottenentwicklung eine Gesamtflotte bestehend aus vier typischen Fahrzeugtypen definiert. Wir greifen für die Untersuchung der zukünftigen Fahrzeugentwicklung auf diese vier Fahrzeugtypen zurück:

- Fullsize: Fahrzeug mit Platz für fünf Passagiere und viel Gepäck (z.B. VW Touareg)

- Compact: Fahrzeug mit Platz für vier Passagiere und wenig Gepäck (z.B. VW Golf)

- Micro: Fahrzeug mit Platz für zwei plus zwei Passagiere oder zwei Passagiere und Gepäck, hauptsächlich im urbanen Einsatz (z.B. Smart)

- 3-Wheeler: Ein- oder Zweisitzer in Leichtbauweise für urbanen Verkehr (e.g. Opel RAK-e gelten zulassungsrechtlich als Motorrad oder Kleinmotorwagen bis $550 \mathrm{~kg}$ (Ausweiskategorie B1)

In all diesen Kategorien sind bereits heute Elektrofahrzeuge angekündigt; zum Beispiel plant allein Toyota für jede der vier Kategorien Elektrofahrzeuge (siehe Abbildung 22).

Unter «3-Wheeler» wird eine neue Fahrzeugklasse verstanden, die nicht gezwungenermassen drei Räder haben muss. Die Fahrzeuge sind in den EU-Klassifizierungen L6e (leicht) und L7e (schwer) definiert, welche auch für «Quads» (vierrädrige Motorräder) gelten. Das Maximalgewicht (ohne Batterie und Zuladung) ist auf 400 Kilogramm beschränkt. 

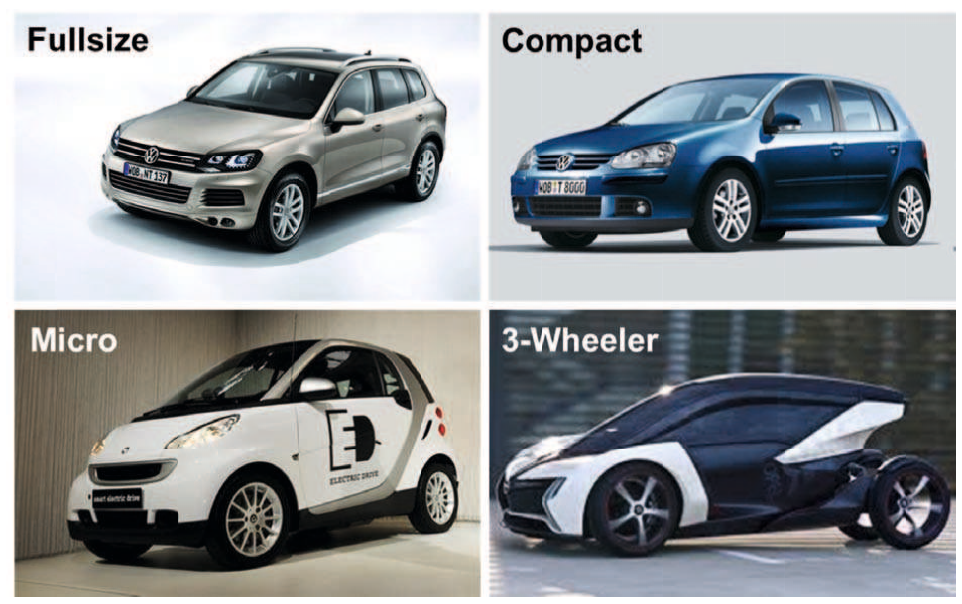

Abbildung 22: Fahrzeugtypen, wie sie in dieser Studie unterschieden werden.

Das erste Fahrzeug dieser Art am Markt ist der Renault Twizy (Markteinführung im Frühjahr 2012). Fortgeschrittene Studien wurden präsentiert von Audi (Urban Concept), Opel (RAKe) und Volkswagen (Nils). Die Fahrzeuge erreichen Spitzengeschwindigkeiten von 90 bis 130 Stundenkilometer bei einer Maximalleistung von 15 Kilowatt (Abbildung 23).
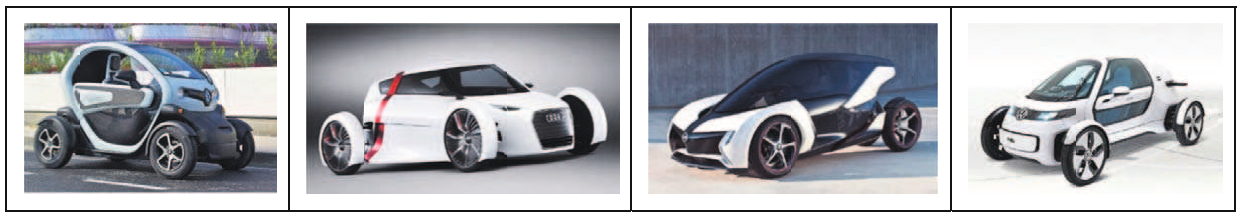

Abbildung 23: Typische Fahrzeuge der L7e Klasse, die in der Studie 3-Wheeler genannt werden: Renault Twizy, Audi Urban Concept, Opel RAKe, Volkswagen Nils (von links nach rechts).

\subsubsection{Gegenwärtiger Stand der Technologie}

Die Fahrzeuge am heutigen Markt sind innerhalb einer Klasse bezüglich Form, Gewicht, Materialien und Fertigungstechnik sehr ähnlich. Es handelt sich um Produkte mit einem sehr hohen industriellen Reifegrad. Es dürften keine radikalen Änderungen zu erwarten sein; Anpassungen erfolgen eher in kleinen Schritten. 


\subsubsection{Entwicklungspfad der Fahrzeuge}

Die Autos wurden lange Zeit immer schwerer und stärker, bis sich vor etwa vier Jahren zuerst eine Stabilisierung, gefolgt von einer leichten Trendwende, abgezeichnet hat. Durch verbesserte Konstruktionen, Werkstoffe und Produktionsverfahren rechnet die Studie daher mit einer leichten Gewichtsabnahme (ein Prozent jährlich bis 2020, 0,5 Prozent in den darauffolgenden Jahren).

Das Raumvolumen der Fahrzeuge (abgeschätzt über die Frontfläche) wird über die Jahre und für die betreffenden Fahrzeugklassen als konstant angenommen. Hingegen werden Verbesserungen beim Luft- und Rollwiderstand erwartet, damit die Anforderungen an verringerten Energieverbrauch und die Einhaltung von verschärften $\mathrm{CO}_{2}$-Emissionszielwerten erfüllt werden können.

\subsubsection{Annahmen der Studie für die Entwicklung der Fahrzeugparameter}

\section{Masse (Glider)}

Der Ausgangspunkt liegt bei 1850 Kilogramm für die Fullsize-Fahrzeugklasse, 1250 Kilogramm für die Kompakt-Klasse, 850 Kilogramm für die Micro-Klasse und 350 Kilogramm für die 3-Wheeler-Klasse.

Durch konstruktive Optimierungen und vermehrten Einsatz von Leichtbaumaterialien wird in der Studie bis 2020 eine durchschnittliche jährliche Reduktion des Glider-Gewichts von einem Prozent angenommen, von 2020 bis 2035 von einem halben Prozent Gewichtsreduktion pro Jahr und von 2035 bis 2050 noch von einem Viertelprozent pro Jahr.

\section{Frontfläche}

Es wird angenommen, dass die Frontfläche innerhalb der jeweiligen Fahrzeugkategorie über die Jahre konstant bleibt. Für die Fullsize-Klasse wird eine Frontfläche von 2,78 Quadratmeter angenommen (Beispiel: VW Touareg), eine Frontfläche von 2,22 Quadratmeter gilt für die Kompaktklasse (Beispiel: VW Golf VI), eine Frontfläche von 2,0 Quadratmeter für die Microklasse (Beispiel: Smart Fourtwo) und für die Klasse 3-Wheelers wird eine Frontfläche von 1,44 Quadratmeter angenommen (Beispiel: Opel RAKe). 


\begin{tabular}{|c|c|c|c|c|c|c|}
\hline \multirow{4}{*}{$\begin{array}{l}\text { Gesamtmasse } \\
\text { inkl. Drivetrain } \\
\text { und Batterie }\end{array}$} & \multicolumn{2}{|c|}{ Einheit } & 2012 & 2020 & 2035 & 2050 \\
\hline & \multirow[t]{3}{*}{$\mathrm{kg}$} & ICE/HEV & 1400 & 1288 & 1191 & 1140 \\
\hline & & PHEV & 1550 & 1433 & 1332 & 1276 \\
\hline & & BEV & 1650 & 1543 & 1451 & 1403 \\
\hline \multirow{3}{*}{$\begin{array}{l}\text { davon Masse } \\
\text { Chassis/Glider }\end{array}$} & \multirow[t]{3}{*}{$\mathrm{kg}$} & ICE/HEV & 1250 & 1153 & 1070 & 1030 \\
\hline & & PHEV & 1250 & 1153 & 1070 & 1030 \\
\hline & & BEV & 1250 & 1153 & 1070 & 1030 \\
\hline \multirow[t]{3}{*}{ Frontfläche $\mathrm{A}$} & \multirow[t]{3}{*}{$\mathrm{m}^{2}$} & ICE/HEV & 2,22 & 2,22 & 2,22 & 2,22 \\
\hline & & PHEV & 2,22 & 2,22 & 2,22 & 2,22 \\
\hline & & BEV & 2,22 & 2,22 & 2,22 & 2,22 \\
\hline \multirow{3}{*}{$\begin{array}{l}\text { Luftwiderstands- } \\
\text { beiwert cw }\end{array}$} & \multirow[t]{3}{*}{-} & ICE/HEV & 0,31 & 0,29 & 0,27 & 0,26 \\
\hline & & PHEV & 0,31 & 0,29 & 0,27 & 0,26 \\
\hline & & BEV & 0,31 & 0,29 & 0,27 & 0,26 \\
\hline \multirow{3}{*}{$\begin{array}{l}\text { Rollwiderstands- } \\
\text { beiwert } \mathrm{cr}\end{array}$} & \multirow[t]{3}{*}{-} & ICE/HEV & 0,011 & 0,010 & 0,009 & 0,008 \\
\hline & & PHEV & 0,011 & 0,010 & 0,009 & 0,008 \\
\hline & & BEV & 0,011 & 0,010 & 0,009 & 0,008 \\
\hline
\end{tabular}

Tabelle 18: Wichtigste Fahrzeugparameter.

\section{Aerodynamik}

Die Ausgangswerte für die Aerodynamik (Luftwiderstandskoeffizient) wurden von typischen heutigen Fahrzeugen übernommen: Fullsize und Micro 0,35, Compact 0,31, 3-Wheeler 0,25.

Für die zukünftige Entwicklung wurde eine Verbesserung des Luftwiderstandskoeffizienten um 0,02 bis 2020 , eine weitere Verbesserung um 0,02 bis 2035 , und eine Verbesserung um 0,01 bis 2050 angenommen. Für die Kompaktklasse ergeben diese Annahmen einen Wert von 0,26 im Jahr 2050, gleich wie beispielsweise ein heutiger Toyota Prius.

\section{Rollwiderstand}

Die Ausgangswerte für den Rollwiderstand wurden angenommen als: Fullsize 0,012, Compact und Micro 0,011, 3-Wheeler 0,010.

Für die zukünftige Entwicklung wurde eine Verbesserung des Rollwiderstands um 0,001 bis 2020, eine weitere Reduktion um 0,001 bis 2035 und nochmals eine Reduktion um 0,001 bis 2050 angenommen. Die Annahmen für den Fortschritt sind begründet in der prinzipiellen technischen Machbarkeit von rollwiderstandsarmen Reifen. Die Reduktion des Rollwiderstands kann jedoch Einschrän- 
kungen bei der Kurvenhaftung oder beim Bremsverhalten zur Folge haben, sodass ein Kompromiss zwischen Energieeffizienz und Sicherheit gefunden werden muss (siehe Tabelle 18).

Für das Jahr 2012 beziehen sich alle Ausgangsparameter auf die in Abbildung 22 dargestellten Fahrzeuge. Eine Extrapolation der Parameter kann als spekulativ angesehen werden, weshalb Abbildung 24 zur Plausibilisierung einige Beispiele von Fahrzeugen und deren Parameter zeigt. Die Abbildung zeigt, dass fast alle in der Studie getroffenen Annahmen zur Fahrzeugparameterentwicklung bereits in der Vergangenheit bei Einzelfahrzeugen erreicht wurden.

Es ist interessant zu sehen, dass die Zielgrössen für die Kompaktklasse im Jahr 2035 sehr ähnlich sind wie die Parameter eines Audi A2 (3L), der bereits im Jahr 2001 auf den Markt kam.

\begin{tabular}{|c|c|c|c|c|c|}
\hline & Fahrzeug & $\begin{array}{c}\text { Masse } \\
{[\mathrm{kg}]}\end{array}$ & $\begin{array}{l}\text { Front- } \\
\text { fläche } \\
\mathrm{A}[\mathrm{m} 2]\end{array}$ & $\begin{array}{c}\text { Luftwider- } \\
\text { stand } \\
\text { c_w [-] }\end{array}$ & $\begin{array}{c}\text { Rollwider- } \\
\text { stand } \\
\text { c_r }[-]\end{array}$ \\
\hline & \multirow{29}{*}{\begin{tabular}{|l} 
Mountainbike \\
Scooter \\
Superbike (head down) \\
Superbike (sitting upright) \\
Enduro \\
PAC-Car World Record ETH \\
Zerotracer \\
Acabion \\
Mini-EI 3-wheeler \\
Opel RAK e concept \\
Ligier Microcar \\
Edison2 X-Price 2010 \\
Loremo \\
WW XL1 0.9L-Car (2013) \\
Smart Roadster Coupé \\
Smart Fortwo \\
Audi A2 3L-Version \\
Polo Blue Motion \\
Golf VI 1.2 2010 Bluemotion \\
Golf V 2.0 TDI \\
Toyota Prius II \\
Opel Ampera \\
Touareg Hybrid 2010 \\
Mercedes SLR Mac Laren \\
Porsche 911 Turbo 996 \\
Ferrari Enzo \\
Audi Q7 SUV \\
Maybach Exelero \\
Formula 1 \\
\end{tabular}} & 15 & 0.75 & 0.68 & 0.007 \\
\hline & & 100 & 0.75 & 0.7 & 0.02 \\
\hline & & 200 & 0.69 & 0.48 & 0.02 \\
\hline & & 200 & 0.77 & 0.56 & 0.02 \\
\hline & & 100 & 0.8 & 0.8 & 0.02 \\
\hline & & 35 & 0.45 & 0.075 & 0.008 \\
\hline & & 640 & 1 & 0.19 & 0.02 \\
\hline & & 400 & 0.66 & 0.11 & 0.008 \\
\hline & & 400 & 0.99 & 0.31 & 0.02 \\
\hline & & 380 & 1.44 & 0.25 & 0.008 \\
\hline & & 880 & 1.7 & 0.33 & 0.011 \\
\hline & & 400 & 1.2 & 0.15 & 0.011 \\
\hline & & 590 & 1.2 & 0.22 & 0.011 \\
\hline & & 795 & 1.5 & 0.186 & 0.01 \\
\hline & & 810 & 1.58 & 0.38 & 0.011 \\
\hline & & 850 & 1.95 & 0.37 & 0.011 \\
\hline & & 895 & 2.2 & 0.252 & 0.011 \\
\hline & & 1100 & 2.05 & 0.32 & 0.011 \\
\hline & & 1271 & 2.22 & 0.31 & 0.011 \\
\hline & & 1400 & 2.22 & 0.32 & 0.011 \\
\hline & & 1500 & 2.16 & 0.26 & 0.011 \\
\hline & & 1700 & 2.07 & 0.275 & 0.011 \\
\hline & & 2315 & 2.78 & 0.35 & 0.011 \\
\hline & & 1850 & 2.03 & 0.42 & 0.011 \\
\hline & & 1760 & 2 & 0.37 & 0.011 \\
\hline & & 1450 & 2 & 0.37 & 0.011 \\
\hline & & 2220 & 2.93 & 0.41 & 0.011 \\
\hline & & 2750 & 2.5 & 0.34 & 0.011 \\
\hline & & 600 & 1.6 & 0.6 & 0.011 \\
\hline
\end{tabular}

Abbildung 24: Fahrzeugparameter verschiedener existierender Fahrzeuge als Orientierungshilfe. 


\subsection{Energiebedarf auf Fahrzeugebene}

\subsubsection{Gegenwärtiger Stand der Technologie}

Elektrofahrzeuge haben eine höhere energetische Effizienz als ICE-Fahrzeuge. Dies zahlt sich vor allem im urbanen Verkehr aus, wo Elektromotoren bei sehr unterschiedlichen Lastsituationen hohe Wirkungsgrade aufweisen und die Bremsenergie rekuperieren können.

Im Vergleich mit ICE-Fahrzeugen verringern sich die Effizienzvorteile bei Autobahnfahrten. Durch den hohen Energiebedarf im Autobahnbetrieb reduziert sich die Reichweite beträchtlich und effiziente ICE-Fahrzeuge können in Langstreckenbetrieb ihre Vorteile ausspielen.

Oftmals wird der Energieverbrauch für den Fahrzeugantrieb (Traktionsenergie) mit dem Komfortenergieverbrauch vermischt. Die benötigte Traktionsenergie ist im Sommer und im Winter grundsätzlich gleich (mit Ausnahme von Unterschieden in der Radreibung und der Luftdichte). Im Winter brauchen BEV aber massiv mehr Komfortenergie für die Heizung, was zu einer signifikanten Reduktion der Reichweite führt. Aus diesem Grund wird Traktionsenergie und Komfortenergie in dieser Studie klar separiert.

\subsubsection{Methodik zur Bestimmung des Fahrzeugenergieverbrauchs}

Beim Vergleich von Fahrzeugen, welche sich in Grösse und Antriebsart unterscheiden, sollte der Verbrauch möglichst neutral berechnet werden. Als Vergleichsbasis dient in dieser Studie ein kleines, mittleres und grosses Vehikel mit gleichem Nutzwert, jedoch unterschiedlichem Antrieb. Der Verbrauch realer Fahrzeuge streut breit; deshalb wurden hier virtuelle Fahrzeuge mit jeweils gleicher Form und Grösse, jedoch unterschiedlichem Antriebsstrang und Gewicht verwendet. Ein Elektroauto hat somit die gleichen Eigenschaften wie ein Auto mit Verbrennungsmotor, das Gewicht ist jedoch höher wegen der Batterie. Dies erhöht prinzipiell die notwendige Antriebsenergie am Rad bei gleichen Fahrleistungen.

Die Radantriebsenergie muss entweder durch einen Verbrennungsmotor, Hybridmotor oder Elektromotor erbracht werden. Für den neuen europäischen Fahrzyklus NEFZ, welcher auch in der Schweiz die Grundlage der Verbrauchsmessungen ist, kann die positive Radenergie mathematisch bestimmt werden. 
Verfügt das Fahrzeug über die Möglichkeit, einen Teil der Bremsenergie zurückzugewinnen, so kann diese Rekuperationsenergie von der positiven Radenergie abgezogen und die reine Radantriebsenergie bestimmt werden.

Je nach Wirkungsgrad der jeweiligem Technologie (beschrieben in den nachfolgenden Kapiteln) muss den Motoren unterschiedlich viel Energie zugeführt werden. Die Motorantriebsenergie ist also höher als die für die Fahrleistung massgebende Radenergie.

Zusätzlich zu dieser Motorantriebsenergie braucht ein Fahrzeug Energie für Sicherheits- und Komfortelemente, also für Beleuchtung, Unterhaltung, Scheibenwischer, Lüftung und vor allem Heizung und Kühlung.

Der gesamte Energieverbrauch umfasst die dem Fahrzeug an der Tankstelle bzw. Ladestation zugeführte Energie.

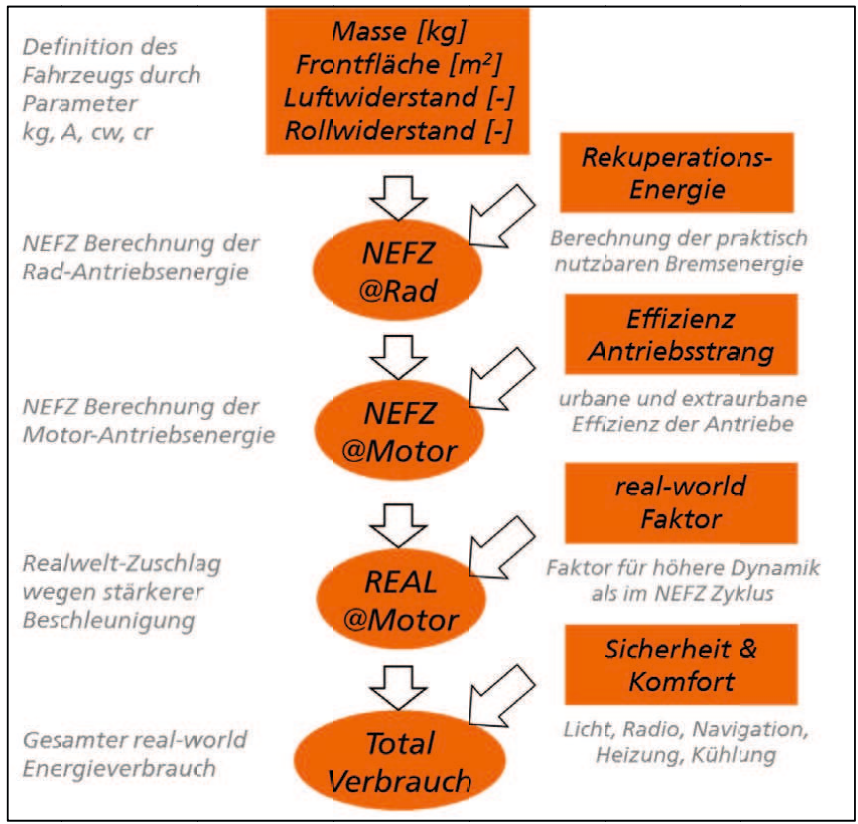

Abbildung 25: Illustration der in der Studie angewandten schrittweisen Berechnung des Fahrzeugverbrauchs mit Differenzierung von Antriebsenergie und Zusatzenergie durch Sicherheits- und Komfortelemente. 


\subsubsection{Entwicklungspfad der Fahrzeuge}

Der Verbrauch eines Fahrzeugs ist gegeben durch seine Konstruktion (Gewicht, Grösse, Form, Reifen) sowie die Effizienz des Antriebssystems und das Fahrprofil. Eine Verbrauchsreduktion kann, bei gleichbleibendem Fahrprofil, durch Verbesserung der Fahrzeugparameter oder durch Verbesserung des Antriebsstrangs erzielt werden. Die Tabelle 19 zeigt das Ergebnis, wenn die oben beschriebenen Parameter eingesetzt werden.

\subsubsection{Annahmen der Studie für die Entwicklung des Fahrzeugenergie- bedarfs}

\section{Energiebedarf im NEFZ}

Der theoretische Energiebedarf für die Traktion an den Rädern wurde für den neuen europäischen Fahrzyklus (NEFZ) berechnet unter Verwendung der Fahrzeugparameter Masse ( $m$ in $[\mathrm{kg}])$, Stirnfläche (A in $\left.\left[\mathrm{m}^{2}\right]\right)$, Luftwiderstandsbeiwert (cw-Wert [-]) und Rollwiderstandsbeiwert (cR-Wert [-]).

\section{Real-World-Zuschlag}

Der NEFZ wird häufig als nicht praxisgerecht eingestuft, weil insbesondere im innerstädtischen Betrieb in der Regel stärker beschleunigt wird als im Zyklus vorgeschrieben (de Haan und Keller 2000). Die Studie rechnet deshalb für den Radenergiebedarf mit 10 Prozent (ICE) bzw. 20 Prozent (PHEV, BEV) Zuschlag im innerstädtischen wie im ausserstädtischen Betrieb. Vom NEFZ-Energiebedarf abgezogen wird derjenige Energieanteil, welcher durch Rekuperation von Bremsenergie zurückgewonnen werden kann. Bei ICE/HEV-Motoren mit Hybridisierung wird für das Jahr 2012 noch mit keiner nennenswerten Rückgewinnung im Flottendurchschnitt gerechnet, ab 2020 jedoch mit 10 Prozent Rückgewinnung (Vollhybridfahrzeuge erreichen höhere Werte, der Anteil von reinen ICE-Fahrzeugen ohne Rekuperationsmöglichkeit ist 2020 jedoch dominant). Bei PHEVFahrzeugen wird bereits ab 2012 mit 40 Prozent und bei BEV-Fahrzeugen mit 50 Prozent Rekuperation gerechnet. Dies sind im Literaturvergleich eher hohe, aber mögliche Werte, welche im Alltag bedingen, dass vorausschauend gefahren wird und keine hohen (negativen) Beschleunigungen auftreten. 


\begin{tabular}{|c|c|c|c|c|c|c|}
\hline Faktor & Einheit & & 2012 & 2020 & 2035 & 2050 \\
\hline \multirow{3}{*}{$\begin{array}{l}\text { Verbrauch NEFZ } \\
\text { (Strom) }\end{array}$} & \multirow{3}{*}{$\begin{array}{l}\mathrm{kWh} / \\
100 \mathrm{~km}\end{array}$} & ICE/HEV & & & & \\
\hline & & PHEV & 8,6 & 9,1 & 8,9 & 8,3 \\
\hline & & BEV & 14,5 & 12,4 & 10,7 & 9,5 \\
\hline \multirow{3}{*}{$\begin{array}{l}\text { Verbrauch NEFZ } \\
\text { (Benzin) }\end{array}$} & \multirow[t]{3}{*}{$\mathrm{l} / 100 \mathrm{~km}$} & ICE/HEV & 6,0 & 4.7 & 3.8 & 3,2 \\
\hline & & PHEV & 1,9 & 1,0 & 0,5 & 0,3 \\
\hline & & BEV & & & & \\
\hline \multirow{3}{*}{$\begin{array}{l}\text { Real-World- } \\
\text { Zuschlagsfaktor } \\
\text { urban/extraurban }\end{array}$} & \multirow[t]{3}{*}{-} & ICE/HEV & 1,10 & 1,10 & 1,10 & 1.10 \\
\hline & & PHEV & 1,20 & 1,20 & 1,20 & 1,20 \\
\hline & & BEV & 1,20 & 1,20 & 1,20 & 1,20 \\
\hline \multirow{3}{*}{$\begin{array}{l}\text { Zusatzverbraucher } \\
\text { Licht, Klima, Radio } \\
\text { etc. }\end{array}$} & \multirow{3}{*}{$\begin{array}{l}\text { kWh/ } \\
100 \mathrm{~km}\end{array}$} & ICE/HEV & 1,5 & 1,4 & 1,3 & 1,3 \\
\hline & & PHEV & 4,5 & 2,9 & 2,3 & 2,3 \\
\hline & & BEV & 4,5 & 2,9 & 2,3 & 2,3 \\
\hline \multirow{3}{*}{$\begin{array}{l}\text { Real-World-Gesamt- } \\
\text { verbrauch Fahrzeug } \\
\text { (Strom) }\end{array}$} & \multirow{3}{*}{$\begin{array}{l}\mathrm{kWh} / \\
100 \mathrm{~km}\end{array}$} & ICE/HEV & & & & \\
\hline & & PHEV & 14,9 & 13,8 & 13,0 & 12,3 \\
\hline & & BEV & 21,9 & 17,7 & 15,2 & 13,8 \\
\hline \multirow{3}{*}{$\begin{array}{l}\text { Real-World-Gesamt- } \\
\text { verbrauch ab } \\
\text { Steckdose (Strom) }\end{array}$} & \multirow{3}{*}{$\begin{array}{l}\mathrm{kWh} / \\
100 \mathrm{~km}\end{array}$} & ICE/HEV & & & & \\
\hline & & PHEV & 16,5 & 14,8 & 13,8 & 13,0 \\
\hline & & BEV & 24,3 & 19,1 & 16,2 & 14,5 \\
\hline \multirow{3}{*}{$\begin{array}{l}\text { Real-World-Gesamt- } \\
\text { verbrauch Benzin) }\end{array}$} & \multirow[t]{3}{*}{$\mathrm{l} / 100 \mathrm{~km}$} & ICE/HEV & 7,5 & 6,0 & 4,8 & 4,1 \\
\hline & & PHEV & 2,3 & 1,2 & 0,6 & 0,3 \\
\hline & & BEV & & & & \\
\hline \multirow{3}{*}{$\begin{array}{l}\text { Elektrische } \\
\text { Real-World-Reich- } \\
\text { weite (bei } 100 \% \\
\text { Entladung) }\end{array}$} & \multirow[t]{3}{*}{$\mathrm{km}$} & ICE/HEV & & & & \\
\hline & & PHEV & 46 & 88 & 211 & 268 \\
\hline & & & 137 & 260 & 618 & 784 \\
\hline
\end{tabular}

Tabelle 19: Energieverbrauch im NEFZ, unter Real-World-Bedingungen, im Jahresschnitt und die theoretischen elektrischen Reichweiten bei Nutzung von 100 Prozent Batteriekapazität.

\section{Zusatzverbraucher}

Zusatzverbrauch für Sicherheit (Licht, Scheibenwischer, Navigation etc.) und Komfort (Heizung und Kühlung), für Werte siehe Kapitel 3.6.3.

\section{Gesamtverbrauch Fahrzeug}

Der Gesamtverbrauch wird aufaddiert aus NEFZ-Verbrauch plus Zuschlag für Real-World-Betrieb plus Zuschlag für Zusatzverbraucher. 


\section{Gesamtverbrauch ab Steckdose bzw. Tankstelle}

Bei den PHEV- und BEV-Fahrzeugen kommen zum Gesamtverbrauch des Fahrzeugs (welcher die Reichweite definiert) noch die Verluste des Ladegerätes dazu, was schlussendlich die ab dem Stromnetz bezogene Energiemenge definiert und damit auch die Betriebskosten und die Auswirkungen der Stromproduktion.

Bei ICE-Fahrzeugen ist der Gesamtverbrauch des Fahrzeugs gleich wie die bezogene Energiemenge an der Tankstelle, weil der Befüllungsprozess als verlustfrei betrachtet werden kann.

\section{Elektrische Reichweite}

Berechnet sich aus dem Real-World-Gesamtverbrauch der PHEV- und BEVFahrzeuge und der Batteriekapazität der Fahrzeuge bei theoretischer hundertprozentiger Entladung (siehe Tabelle 19).

\subsubsection{Schlussfolgerungen}

Technikfolgenabschätzung beinhaltet das Risiko von Schätzfehlern, besonders dann, wenn der Blick weit in die Zukunft gerichtet ist und quantitative Aussagen beinhaltet wie in dieser Studie. Um dem entgegenzuwirken, wurden alle Annahmen so transparent, realistisch und neutral wie möglich getroffen und durch Aufteilung in Teilfaktoren (z.B. Traktionsenergie vs. Komfortenergie) individuell beurteilbar gehalten. Es gilt anzumerken, dass die verschiedenen Antriebssysteme in der gleichen Weise behandelt wurden, sodass beispielsweise Massnahmen zur Gewichtsreduktion, die sowohl bei ICE- als auch bei BEV-Fahrzeugen angewandt werden können, auch bei beiden Antriebstypen in gleichem Mass berücksichtigt wurden.

Grundsätzlich überraschend ist die grosse kumulative Wirkung der Kombination kleiner Verbesserungsmassnahmen bei allen Antriebsformen. Ein Kompaktwagen, der heute 7,5 Liter Benzin pro 100 Kilometer verbraucht, kommt im Jahr 2035 auf einen Verbrauch von 4,8 Liter, was einer Reduktion von mehr als einem Drittel entspricht. Der gleiche Kompaktwagen mit elektrischem Antrieb könnte seinen Strombedarf von heute 24 Kilowattstunden auf 16 Kilowattstunden pro 
100 Kilometer im Jahr 2035 reduzieren, was eine Reduktion von gut 30 Prozent darstellt.

Interessant ist, dass das Verbesserungspotenzial für Elektrofahrzeuge eher kleiner ist als für ICE-Fahrzeuge. Die höheren Effizienzsteigerungen bei ICE-Fahrzeugen können dadurch erklärt werden, dass das Verbesserungspotenzial der Effizienz bei Verbrennungsmotoren grösser ist als bei Elektromotoren. Bei den Elektrofahrzeugen liegt das Verbesserungspotenzial dagegen vor allem bei $\mathrm{Ne}-$ benverbrauchern wie Heizung oder Batteriekonditionierung.

PHEV und BEV haben zwar im Vergleich zum ICE-Antrieb ein etwas geringeres Effizienzverbesserungspotenzial, verbunden mit dem Fortschritt bei der Batteriekapazität von 100 auf $300 \mathrm{Wh} / \mathrm{kg}$ führt dies aber bei gleichbleibendem Batteriegewicht (100 bzw. 300 Kilogramm) zu einer wesentlichen Erhöhung der theoretischen elektrischen Reichweite von rund 50 auf 200 Kilometer für das PHEV und von rund 150 auf 600 Kilometer für das BEV. 


\section{Lebenszyklusanalyse}

\subsection{Ziel und Rahmen der Lebenszyklusanalyse}

Die Studie soll die Umweltauswirkungen der im voranstehenden Kapitel entworfenen Mobilitätsszenarien von heute bis 2050 mit mehr oder weniger Elektrofahrzeugen bestimmen, um die Chancen und Risiken der Elektromobilität in der Schweiz abzuschätzen. Die Ergebnisse sollen als Basis für eine Versachlichung der öffentlichen Diskussion dienen.

Dies verlangt eine Lebenszyklusperspektive, also eine Betrachtung von Produktion, Nutzung und Entsorgung der Fahrzeuge, die für die Mobilität eingesetzt werden. Eine ausschliessliche Betrachtung der Nutzung von Fahrzeugen greift zu kurz, da Elektrofahrzeuge kaum Emissionen in der Nutzungsphase verursachen, dafür aber die Strombereitstellung bezüglich Emissionen äusserst relevant sein kann. Auch eine "Well-to-Wheel-Betrachtung», in der die Vorketten der Energiebereitstellung, nicht aber die Herstellungsprozesse der Fahrzeuge berücksichtigt werden, kann vor allem für elektrische Fahrzeuge die Umweltbelastungen deutlich zu niedrig einschätzen. Entsprechend werden komplette Lebenszyklusanalysen (= Ökobilanzen) «von der Wiege bis zur Bahre» bzw. - im Falle von Recycling von Materialien am Lebensende eines Produktes - «von der Wiege bis zur Wiege» vorgenommen. Dabei werden alle für das System nötigen Prozesse und Produkte berücksichtigt, die bezüglich Masse, Energie oder potenzieller Umweltwirkung als relevant gelten. Dieser Grundsatz wird soweit möglich auch auf Materialien angewandt, die nach dem Lebensende rezykliert werden. Es gibt also - zumindest im Vordergrundsystem, das die Fahrzeugproduktion, Nutzung und Entsorgung beinhaltet - keinen sogenannten CutOff, bei dem Materialien ohne Gut- oder Lastschrift einfach aus dem System entlassen werden. Diese Cut-Offs werden vermieden, indem das System um die Wiederaufbereitungsprozesse erweitert wird und mit den zurückgewonnenen Materialien primäre Inputmaterialien ersetzt werden. Im Hintergrundsystem, das Daten aus der Ecoinvent-v2.2-Datenbank verwendet (Frischknecht, Jungbluth et al. 2004), konnten die Cut-Offs leider nicht entfernt werden. Dadurch werden Umweltbelastungen im Hintergrundsystem überschätzt für Produkte, die überdurchschnittliche Recyclingquoten erreichen und unterschätzt für Produkte, die unterdurchschnittlich rezykliert werden. In einigen Fällen - z.B. bei der Stromübertragung oder der Stromerzeugung mit PV oder Windkraft - muss deshalb 
davon ausgegangen werden, dass die Abweichungen zulasten dieser Technologien relativ gross sind.

Um das Ziel der Studie zu erreichen, werden modular aufgebaute Ökobilanzen zu vier Zeitpunkten (2012, 2020, 2035 und 2050) erstellt. Die erste Stufe umfasst die Fahrzeuge, deren Betrieb und Entsorgung bezogen auf einen mit dem Fahrzeug zurückgelegten Kilometer. Diese Bilanzen basieren vor allem auf Lebenszyklusanalysen, die von der Empa gemäss ISO 14 040/14 044 erstellt wurden. Die Hauptquellen, die verwendet werden, sind Althaus und Gauch (2010), Althaus et al. (2012), und die Aktivitäten im THELMA-Project (THELMA 2012), daraus insbesondere Habermacher (2011). Wir untersuchen Fahrzeuge aus drei Grössenklassen mit jeweils drei Antriebstechnologien. Zusätzlich betrachten wir bei den Elektrofahrzeugen die Kategorie der Dreiräder, die für kleine, leichte Fahrzeuge wie den SAM stehen, die aber - wie der Renault Twizzy - auch vier Räder haben können. Die untersuchten Fahrzeuge sind in Abbildung 26 ersichtlich.

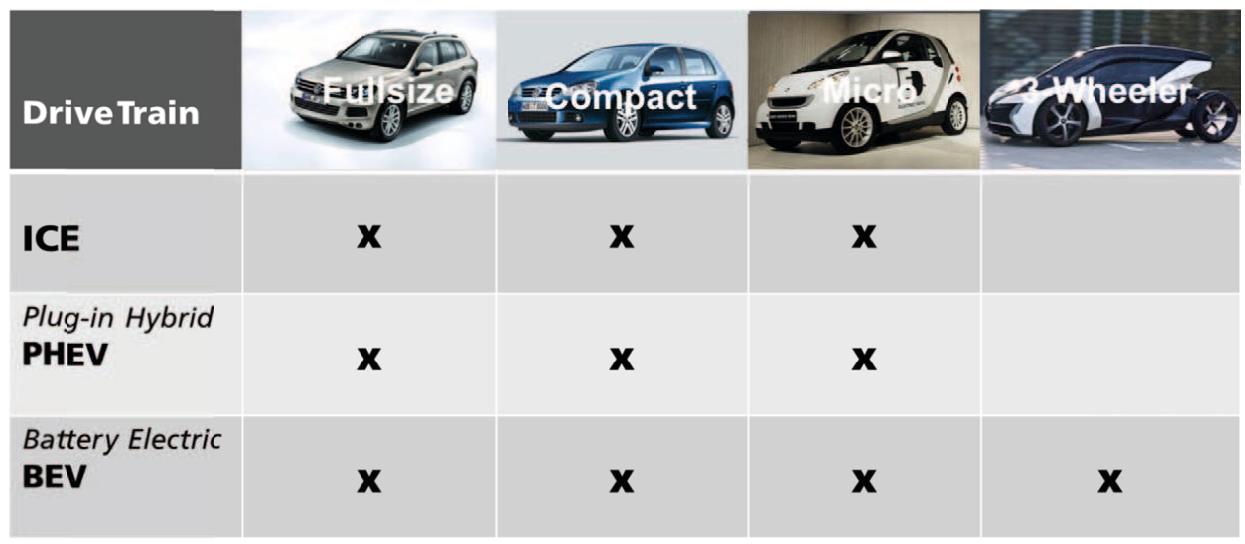
Abbildung 26: In der LCA berücksichtigte Grössenklassen, Antriebsstrang- technologien und deren Kombinationen.

Auf Basis dieser ersten Stufe lassen sich die Auswirkungen des technischen Fortschritts beurteilen und es können Fahrzeuge derselben Kategorie mit unterschiedlichen Antriebssträngen verglichen werden. Bei solchen Vergleichen ist aber zu beachten, dass die bilanzierten Fahrzeuge jeweils für durchschnittliche 
Fahrzeuge einer Kategorie stehen und dass mit diesen Resultaten also nicht beurteilt werden kann, ob ein bestimmtes Elektroauto «besser» oder «schlechter» abschneidet als ein spezifisches Benzinauto. Man kann auf dieser Stufe auch unterschiedliche Fahrzeugkategorien untereinander vergleichen, muss sich aber bewusst sein, dass ein 400 Kilogramm leichter Zweisitzer mit knapp 100 Kilometern Reichweite kein vollwertiger Ersatz eines zwei Tonnen schweren SUV mit fünf Plätzen ist, mit dem man einen Anhänger ziehen kann. Auf der zweiten Stufe werden für die drei Szenarien die Gesamtzahl der zurückgelegten MIV-Personenkilometer in der Schweiz in jedem der vier Jahre bilanziert und die daraus resultierende gesamte Umweltbelastung pro Jahr berechnet.

Umweltauswirkungen können mit unterschiedlichen Methoden bestimmt werden. Grundsätzlich unterscheidet man Methoden, die Ergebnisse für bestimmte Umweltprobleme berechnen (Midpoint-Methoden) von Methoden, die Auswirkungen auf bestimmte Schutzgüter bestimmen (Endpoint-Methoden) und von Methoden, die den Abstand zum angestrebten Umweltziel (Distance-to-Target-Methoden) berechnen. Die Mid- und Endpoint-Methoden sind naturwissenschaftlich basiert und versuchen Wirkungen möglichst wertfrei zu bestimmen, während die Distance-to-Target-Methoden sich an politisch gesetzten Umweltzielen orientieren, die nicht unbedingt etwas mit dem effektivem Schadenspotenzial zu tun haben: Die für die Schweiz entwickelte «Methode der ökologischen Knappheit», die Ergebnisse in Umweltbelastungspunkten (UBP) ausdrückt, gehört dazu. MidpointMethoden umfassen unter anderem die Klimaerwärmung, den stratosphärischen Ozonabbau, die troposphärische Ozonbildung (Sommersmog), Versauerung, Überdüngung, Humantoxizität und Ökotoxizität. Die Indikatoren für die unterschiedlichen potenziellen Probleme sind untereinander nicht vergleichbar und widersprechen sich in vergleichenden Studien oft gegenseitig, was eine Interpretation der Ergebnisse schwierig macht. Dieses Problem war der Anlass zur Entwicklung von Endpoint-Methoden, die ausgehend von den Midpoint-Ergebnissen mit Modellrechnungen bestimmen, wie gross die Wirkung auf jedes der drei Schutzgüter «menschliche Gesundheit», «Ökosystemqualität» und «Ressourcenqualität» ist. Ein direkter Vergleich der drei resultierenden Indikatoren ist zwar auch nicht möglich, doch sind die Schutzgüter weniger abstrakt als die Midpointindikatoren und ihre relative Bewertung fällt dadurch leichter. In dieser Studie verwenden wir einen Midpointindikator für das Treibhauspotenzial (Global Warming Potential, GWP) nach IPCC 2007 (IPCC 2007), den wir mit der Einheit " $\mathrm{CO}_{2}$ eq» messen und die drei Endpointindikatoren «menschliche Gesundheit», «Ökosystemqualität» und «Ressourcenqualität», berechnet mit der ReCiPe-Methodik (Goedkoop et al. 2009). 


\subsection{Lebenszyklusinventardaten}

\section{Fahrzeugproduktion}

Obwohl die Fahrzeugproduktion einen wichtigen Teil der Ökobilanz von Mobilität ausmacht und obwohl die meisten grossen Autohersteller für interne Zwecke Ökobilanzdaten benutzen, sind nur wenige Daten zur Fahrzeugproduktion öffentlich verfügbar. Die Fahrzeugdaten in vielen Ökobilanzen basieren heute auf einer Studie von Volkswagen, die Ergebnisse einer Analyse eines Golf IV der 1990er-Jahre publiziert (Schweimer und Levin 2002). Diese Studie ist auch eine der Grundlagen für die Fahrzeugproduktion in unserem Bericht und liefert die Daten für die Produktionsaufwendungen und -emissionen. Wir haben darin die Komponenten des Antriebsstranges identifiziert und von den Komponenten getrennt, die motorentechnologieunabhängig sind. Letztere bezeichnen wir insgesamt als «Glider». Die Materialzusammensetzung des Gliders wurde für die Ökobilanz von allen Fahrzeugen verwendet, während die des Antriebsstrangs nur für die thermischen Autos gebraucht wird.

Im Modell der elektrischen Fahrzeuge wird ein elektrischer Antriebsstrang abgebildet, dessen Daten vor allem auf direkten Angaben eines Schweizer Produzenten solcher Komponenten (Brusa) basieren (Habermacher 2011). Die Daten zu der Lithium-Ionen-Batterie, die im Fahrzeug eingesetzt wird, basieren auf einer umfangreichen Studie der Empa (Notter, Gauch et al. 2010), in der sämtliche Schritte des Herstellungsprozesses einzeln analysiert und bilanziert wurden. Die Massen sowie andere wichtige Parameter der Fahrzeuge und deren Hauptkomponenten sind in Kapitel 4 zusammengestellt. Die wichtigsten Eigenschaften der Batterien sind in Kapitel 3.2 zu finden, detaillierte technische Angaben finden sich im Anhang. Die Daten für die Herstellung der Fahrzeuge mit ihren Komponenten entsprechen damit denen, die demnächst in ecoinvent Version 3 publiziert werden. Die Hintergrunddaten (Strom- und Treibstoffbereitstellung, Produktion von Materialien etc.) stammen für die Berechnungen in diesem Bericht aber noch aus ecoinvent Version v2.2, da zur Zeit der Berechnungen die Version 3 noch nicht verfügbar war.

\section{Fahrzeugnutzung}

Die zur Nutzung von Fahrzeugen benötigte Energie kann sowohl für ICE und HEV als auch für EV und PHEV - je nach Quelle und betrachteter Umweltwir- 
kung - einen sehr relevanten Beitrag zu den Ökobilanzergebnissen leisten. Der Energiebedarf für die Fortbewegung eines Fahrzeuges hängt im Wesentlichen von der Masse und dem Widerstand (Roll- und Luftwiderstand) des Fahrzeugs sowie vom Fahrprofil ab, in dem das Fahrzeug benutzt wird. Um den Treibstoffoder, im Falle eines Elektroautos, den Strombedarf zu errechnen, braucht man zusätzliche Informationen zum Wirkungsgrad des Antriebsstranges des Fahrzeuges. In vielen Studien wird als Energiebedarf nur der Strombedarf des Fahrzeuges zur Fortbewegung in einem normierten Fahrzyklus, dem neuen europäischen Fahrzyklus (NEFZ) berücksichtigt. Die NEFZ-Verbräuche der untersuchten Fahrzeuge sind zusammengefasst im Kapitel 4.2.4 dargestellt und im Detail im Anhang 3 zu finden. Zu deren Berechnung wurden die Wirkungsgrade des Antriebsstranges gemäss Anhang 3 verwendet. Bei PHEV wird mit einem Anteil von 60 Prozent (2012) bis 90 Prozent (2050) gearbeitet.

Der zukünftige Energiebedarf der Benzinfahrzeugflotte wird dabei basierend auf Annahmen zur zukünftigen Technologieentwicklung und Flottenzusammensetzung abgeschätzt. Zur Validierung wurden die so berechneten Verbräuche mit den $\mathrm{CO}_{2}$-Emissionszielen des Bundesrates für die Jahre 2015 bis 2035 verglichen (siehe Abbildung 22). Dabei wurde berücksichtigt, dass diese Ziele für die gesamte Flotte gelten, also die Elektromobile zu deren Erreichen beitragen und somit der Flottenschnitt der Verbrennungsmotorfahrzeuge über den vereinbarten Zielen liegen kann. Diese Art der Berechnung stellt sicher, dass die Abschätzung zukünftiger Umweltwirkungen nur unwesentlich dadurch beeinflusst wird, dass die gesamte Verbrennerflotte, zu der auch die Diesel-, LPG- und Gasfahrzeuge zählen, durch Benzinautos repräsentiert wird.

Da der NEFZ nur sehr langsame Beschleunigungen kennt, unterschätzt man damit den Energiebedarf des Fahrzeuges in einer durchschnittlichen realen Fahrsituation um 5 bis 10 Prozent. Ausserdem werden dadurch zwei weitere Bereiche vernachlässigt, deren Berücksichtigung den Strombedarf deutlich erhöht: Ladeverluste und Energiebedarf für Komfort und Sicherheit. Ladeverluste liegen typischerweise im Bereich von 5 bis 15 Prozent. Der Energiebedarf für Komfort und Sicherheit hängt von der Fahrzeuggrösse ab und liegt heute bei Elektrofahrzeugen im Bereich von 2,25 bis $2,75 \mathrm{kWh} / 100 \mathrm{~km}$, bei Verbrennern muss mit ca. 0,2 l/100 km Zusatzverbrauch gerechnet werden. Die Werte sind in Anhang 4 zusammengestellt und aufgrund der ebenfalls in Anhang 4 aufgeführten Annahmen berechnet. In Zukunft dürften sowohl die Ladeverluste als auch der Bedarf für Komfort und Sicherheit reduziert werden, Letztere zum Beispiel durch den Einsatz von Wärmepumpen statt Widerstandsheizungen oder durch 
thermische Isolation der Fahrgastzelle. Die spezifischen Daten, mit denen für die Ökobilanzierung gerechnet wurde, finden sich ebenfalls im Anhang 4. Neben den Abgasen verursachen alle Autos, auch die elektrischen, Emissionen durch Abrieb von Reifen, Strasse und Bremsen. Bei diesen Emissionen, die mit den ecoinvent-Daten abgebildet werden, wird bei Elektroautos und Hybriden eine Reduktion des Bremsabriebs berücksichtigt, die durch die Möglichkeit des rekuperativen Bremsens erreicht wird. Lärmemissionen, die bekanntermassen einen sehr relevanten Beitrag zu Gesundheitseffekten des Autofahrens leisten können, werden aufgrund noch fehlender Daten nicht berücksichtigt. Da Lärmemissionen von Autos bis rund 50 Stundenkilometer vom Anteil des Motorengeräusches dominiert werden (bei höherer Geschwindigkeit dominieren Roll- und Strömungsgeräusche), ist zu erwarten, dass vor allem in städtischen Gebieten ein signifikanter Unterschied bezüglich Lärmemission eines Elektro- und eines Verbrennungsmotorautos festzustellen ist.

Ebenfalls zur Nutzung gehört der Fahrzeugunterhalt. Dabei fällt vor allem der Ersatz der Antriebsbatterie bei jedem zweiten Fahrzeug von 2012 ins Gewicht, der, so die Annahme, ab 2020 nicht mehr nötig sein wird.

\section{Entsorgung der Fahrzeuge}

Obwohl viele Fahrzeuge aus der Schweiz mit über 150000 Kilometern auf dem Zähler nach Osteuropa oder in den Süden exportiert und dort weiter benutzt werden, gehen wir - in Übereinstimmung mit den meisten anderen LCA-Studien - davon aus, dass die Fahrzeuge in der Schweiz fachgerecht entsorgt werden. Damit wird die Umweltbelastung des Fahrens pro Kilometer einerseits überschätzt, weil das Fahrzeug in Realität länger lebt als angenommen. Andererseits kann die Belastung durch die Fahrzeugentsorgung in Ländern, in denen kein formelles Recycling existiert, auch grösser sein als durch unser Modell berechnet. Als Entsorgungspfad wird in dieser Studie ein weitgehendes Recycling angenommen. Die Rückgewinnungsquoten sind abhängig von den jeweiligen Materialien und deren Verwendung im Fahrzeug. Die in Althaus und Gauch (2010) verwendeten Zahlen wurden übernommen. So wird zum Beispiel für das Recycling von Aluminium, das im Glider eines Fahrzeuges verbaut ist, eine Recyclingrate von über 90 Prozent angenommen, während für Aluminium, das in den Zellen der Batterien steckt, ein hundertprozentiger Verlust bilanziert wird. Dies, weil Li-Batterien in Kupferhütten rezykliert werden, in denen das Aluminium als Oxid in die Schlacke gelangt und nicht mehr als Metall zur Verfügung steht. Obwohl es durchaus möglich ist, dass in Zukunft andere Prozesse für das Batterierecycling 
angewandt werden, mit denen auch das Aluminium zurückgewonnen werden kann, gehen wir in dieser Studie auch in den zukünftigen Szenarien von dieser für die Batterien unvorteilhaften Annahme aus. Die zurückgewonnenen Materialien werden dem System gutgeschrieben.

\subsection{Lebenszyklusinventardaten zur Energie- bereitstellung}

In dieser Studie benutzen wir für die Energiebereitstellung im Jahr 2012 die Strommixdaten (Verbrauchsmix) aus ecoinvent v2.2 sowie die entsprechenden Daten zur Bereitstellung von Benzin. Während die Daten für die Benzinbereitstellung über die Jahre als konstant angenommen werden (die stetige Zunahme des Aufwands zur Gewinnung von fossilen Energieträgern, die mit höherem Energieund Wasserbedarf und damit mit höherer Umweltbelastung verbunden ist, wird vernachlässigt), wird der Strommix gemäss den Prognosen des BFE im Szenario «neue Energiepolitik» (siehe Kap. 2.6) über die Jahre verändert (Abbildung 27).

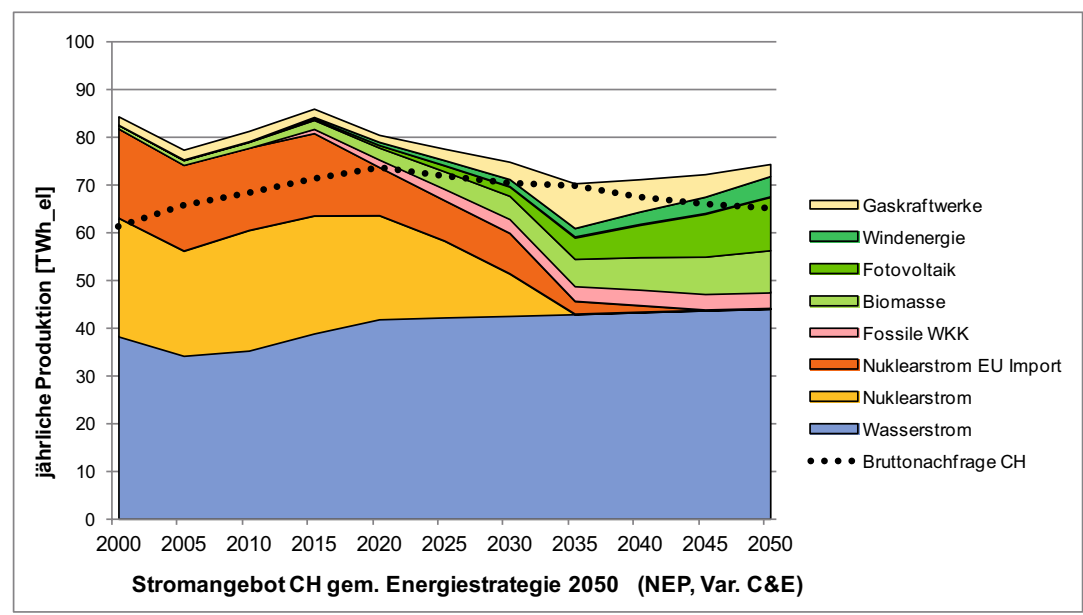

Abbildung 27: Energiestrategie des Bundesrates zur Entwicklung des Stromangebotes in der Schweiz (Flächenanteile) und Elektrizitätsverbrauch $\mathrm{CH}$ (gepunktete schwarze Linie). Zur Verhinderung einer Stromlücke sollen die wegfallenden Anteile aus der Atomenergie durch Gaskraftwerke gedeckt werden. 
Gemäss der vom Bundesrat im April 2012 vorgestellten Prognose der Zusammensetzung des zukünftigen Strommixes in der Schweiz ergibt sich ein Entwicklungspfad mit stark veränderlichen Umweltauswirkungen über die nächsten Jahrzehnte. Abbildung 29 zeigt die $\mathrm{CO}_{2}$-Emissionen des zukünftigen Strommixes. Auffällig ist die bedeutende Auswirkung der geplanten Gaskraftwerke ab 2020, welche zu einem fast fünffach höheren $\mathrm{CO}_{2}$-Impakt pro Kilowattstunde führen wird als heute. Die zunehmende Versorgung mit erneuerbaren Energien führt dann bis 2050 zu einem Rückgang dieser Emissionen.

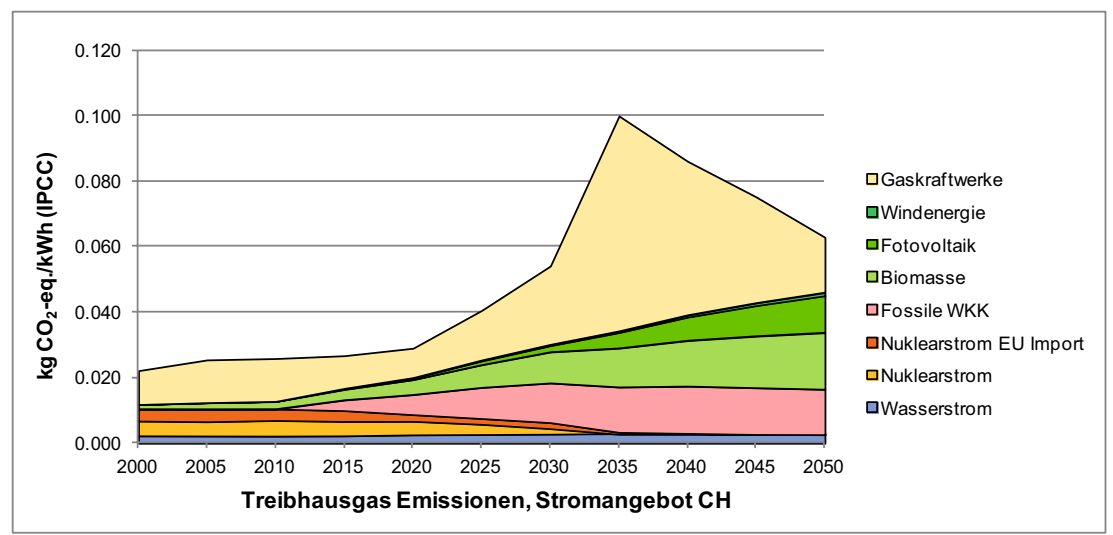

Abbildung 28: $\quad \mathrm{CO}_{2}$-Impakt des schweizerischen Strommixes gemäss Energiestrategie des Bundesrates.

Ein ähnliches Bild zeigt die Analyse der Gesamtumweltauswirkungen (Abbildung 29, Abbildung 30, Abbildung 31). Auch hier verschlechtern sich die ökologischen Auswirkungen des Stroms bis 2035 deutlich durch die Einführung von Gaskraftwerken, dafür gehen die Auswirkungen der einheimischen und importierten Nuklearenergieanteile zurück. Nach 2035 nehmen die Anteile neuer erneuerbarer Energien deutlich zu und der Anteil Strom aus Gaskraftwerken nimmt ab (siehe Abbildung 29, 30, 31).

Der globale Strommarkt ist deutlich $\mathrm{CO}_{2}$-lastiger durch die hohen Anteile fossiler Energie. Interessant ist die Tatsache, dass globale Prognosen (World Energy Outlook, siehe IEA 2011) davon ausgehen, dass die $\mathrm{CO}_{2}$-Belastung pro Kilowattstunde Strom rückläufig sein wird (siehe Anhang $A 3$, Abbildung 58). Es ist daher davon auszugehen, dass die Energiesysteme in Batterieherstellerländern (Chile 
und Bolivien für Lithium, China, Japan etc.) und Fahrzeugherstellerländern (Deutschland, USA, China etc.) in Zukunft ebenfalls nachhaltiger wird, was in den Hintergrunddaten dieser Studie nicht berücksichtigt werden konnte.

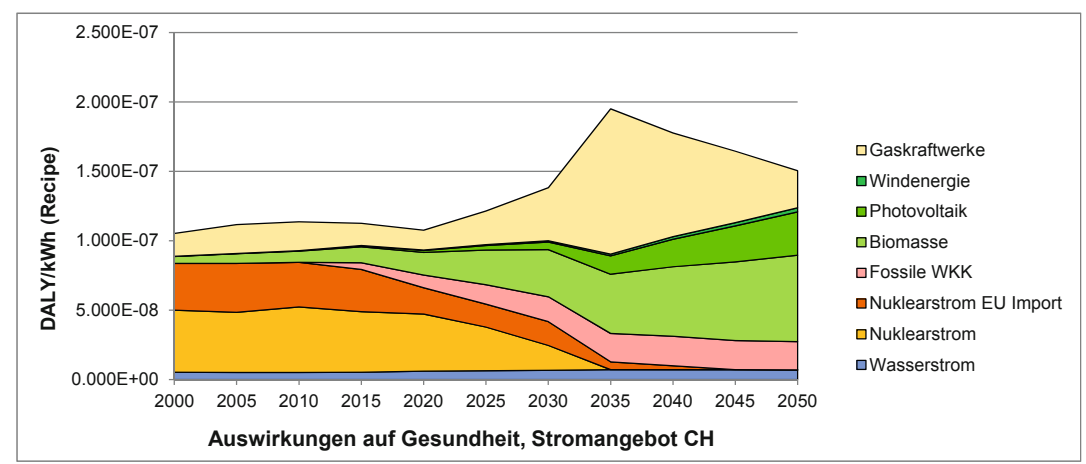

Abbildung 29: Human Health Impact (Auswirkungen auf menschliche Gesundheit nach ReCiPe-Methodik) des schweizerischen Strommixes gemäss Energiestrategie des Bundesrates.

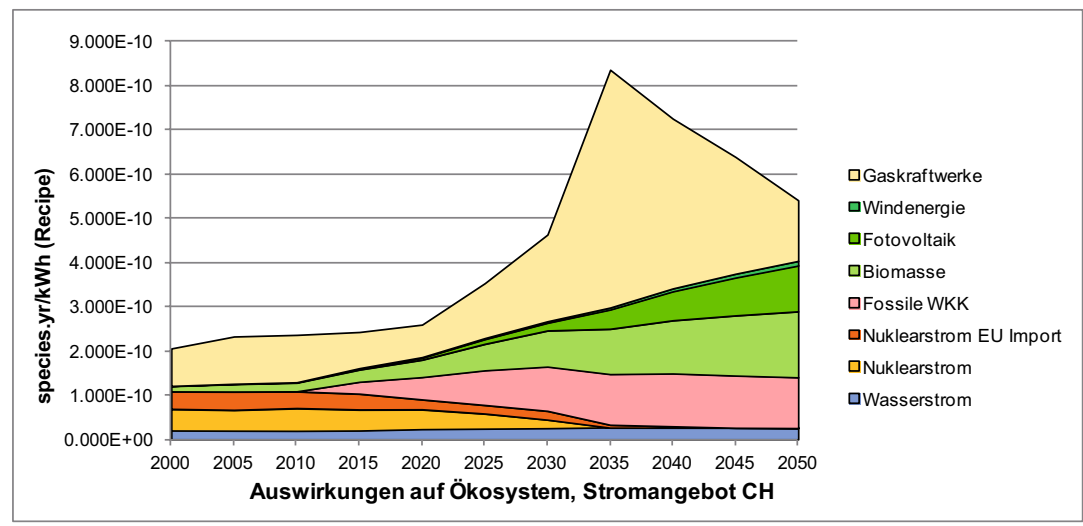

Abbildung 30: Ökosystem-Impakt (Auswirkung auf die Natur nach ReCiPeMethodik) des schweizerischen Strommixes gemäss Energiestrategie des Bundesrates. 


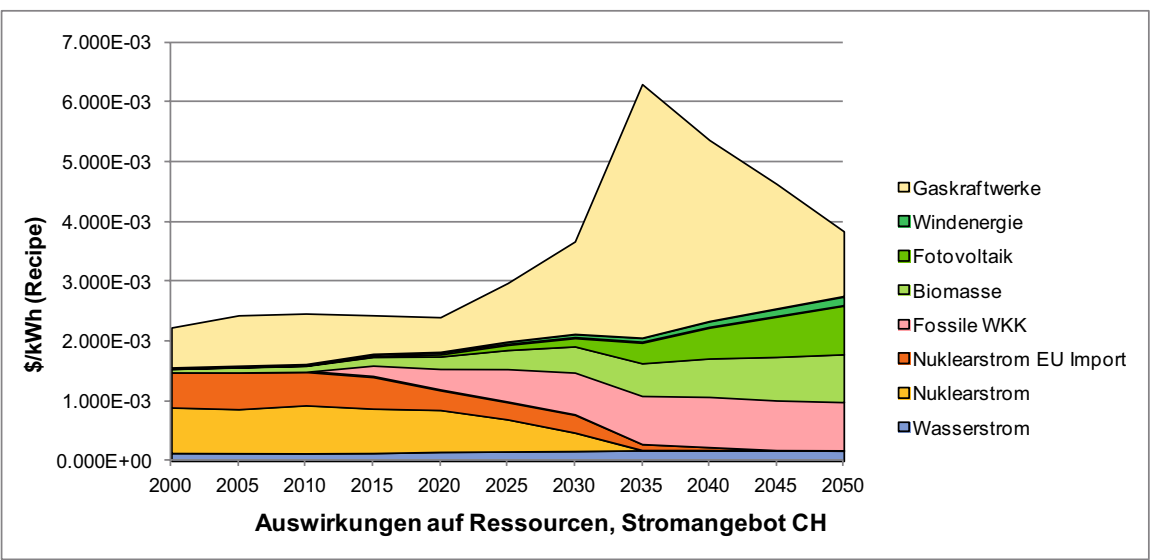

Abbildung 31: Ressourcen-Impakt (Auswirkung des natürlichen Ressourcenverbrauchs nach ReCiPe-Methodik) des schweizerischen Strommixes gemäss Energiestrategie des Bundesrates.

\subsection{Resultate auf Stufe «Fahrzeug»}

Die Treibhausgasemissionen über den ganzen Lebenszyklus eines Fahrzeugkilometers sind in Abbildung 32 für Fahrzeuge der Kompaktklasse dargestellt. Auf den ersten Blick fällt auf, dass bei ICE-Fahrzeugen der grösste Teil der Treibhausgase beim Fahren emittiert wird (hellgrauer Balken), während bei den BEV die Emissionen in die Stromproduktionskette verlagert sind (hellgrüne Balken). Durch den zusätzlichen Produktionsaufwand für die Batterien liegen die Treibhausgasemissionen für die gesamte Fahrzeugproduktion für PHEV und in besonderem Masse für BEV etwa ein Viertel über den Produktionsemissionen für ICE-Fahrzeuge.

Das zukünftige Treibhausgas-Einsparpotenzial ist für die ICE-Fahrzeuge generell höher als für die PHEV und BEV. Elektrofahrzeuge weisen bereits heute niedrige Treibhausgasemissionen auf, welche hauptsächlich durch Produktionsemissionen bestimmt sind (blaue Balken), welche in Zukunft kaum abnehmen werden. Die Abnahme in der $\mathrm{CO}_{2}$-Intensität der Batterieproduktion zwischen heute und 2020 lässt sich mit der Zunahme der Zyklenfestigkeit der Batterien erklären, welche ab 2020 keinen Batteriewechsel im Fahrzeuglebenszyklus 
mehr nötig machen. Die höhere Energiedichte von zukünftigen Batterien führt nicht zu einer Reduktion der Auswirkung der Batterieproduktion, da davon ausgegangen wird, dass die Batteriemasse konstant bleiben wird und dafür die Reichweite steigt.

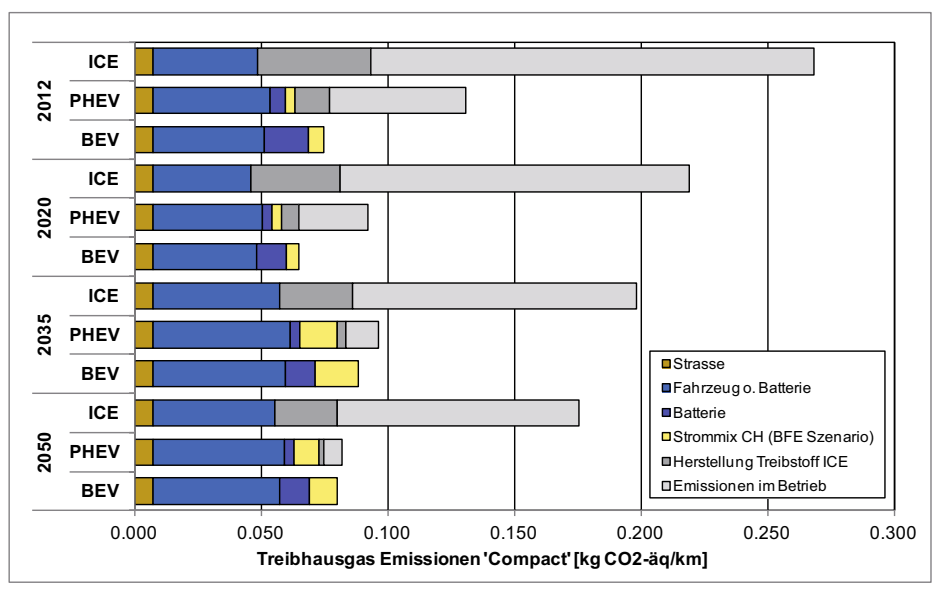

Abbildung 32: Lebenszyklus-Treibhausgasemissionen für die drei untersuchten Antriebsstränge (ICE, PHEV, BEV) und die vier Zeitpunkte (2012/20/35/50) für die Fahrzeugklasse «Kompakt».

Interessanterweise ergibt die Ökobilanzierung für 2020 und 2035 bei den BEV steigende Treibhausgasemissionen, was einerseits durch die höhere $\mathrm{CO}_{2}$-Intensität des gemäss den Energieszenarien für 2035 prognostizierten Strommixes (gelbe Balken) und andererseits durch den zunehmenden energieintensiven Leichtbau (hellblaue Balken) erklärt werden kann. Bei den PHEV bleiben die Ergebnisse praktisch konstant, da sich die Effekte der grösser werdenden elektrischen Reichweite und der steigenden $\mathrm{CO}_{2}$-Intensität des Stromes gegenseitig aufheben. Bei den ICE-Fahrzeugen zeigt sich dagegen eine deutliche kontinuierliche Reduktion der Treibhausgasemissionen über den ganzen betrachteten Zeitraum, welche sich durch die kombinierte Einführung verschiedener effizienzsteigender Massnahmen (Downsizing, Start-Stop-Abschaltung, Hybridisierung etc.) im Massenmarkt ergeben.

Zusammenfassend liegen die Treibhausgasemissionen auf Fahrzeugebene bei elektrischem Antrieb in der Kompaktklasse um 50 bis 70 Prozent niedriger als 
bei entsprechenden ICE-Fahrzeugen, wobei die Differenzen in der Zukunft kleiner werden. Bei den heutigen BEV entstehen bereits 90 Prozent der Treibhausgasemissionen in der Fahrzeugproduktion. Bei den ICE-Fahrzeugen sind es heute 25 Prozent und längerfristig 40 Prozent. Es ist wichtig anzumerken, dass sich diese Werte auf den heutigen und den für die Zukunft prognostizierten Strommix der Schweiz beziehen, welcher im Vergleich zu vielen anderen Ländern sehr $\mathrm{CO}_{2}$-arm ist. Ein für die Elektrofahrzeuge ungünstigeres Bild ergäbe sich bei einem hauptsächlich auf fossilen Energieträgern basierten Strommix.

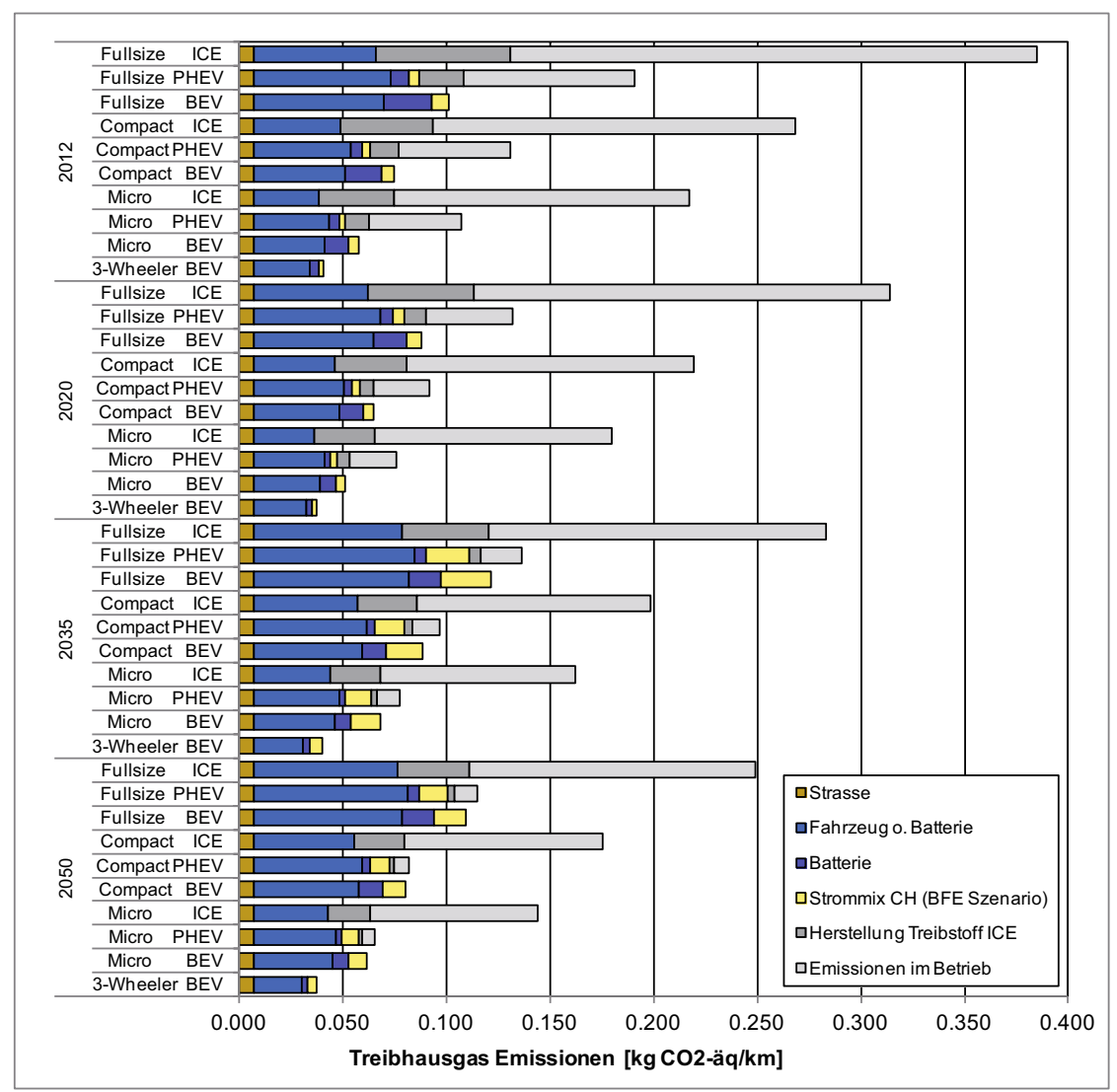

Abbildung 33: Lebenszyklus-Treibhausgasemissionen für die drei untersuchten Antriebsstränge (ICE, PHEV, BEV) und die vier Zeitpunkte (2012/20/35/50) für die vier Fahrzeugklassen (Fullsize, Compact, Micro, 3-Wheeler). 
In Abbildung 33 werden alle vier untersuchten Fahrzeugklassen verglichen. Die Unterschiede in den Ergebnissen für die vier Fahrzeugklassen lassen sich durch die unterschiedlichen Fahrzeuggewichte und Energieverbräuche erklären. Obschon das absolute Einsparpotenzial in der Fahrzeugklasse «Fullsize» am grössten ist, ist die Bedeutung von elektrischem Antrieb für diese Klasse eher gering; gemeinhin wird angenommen, dass grössere Fahrzeugkategorien einen grösseren Teil ihrer Fahrleistung im ausserstädtischen Bereich zurücklegen; auch sind die Batterieladezeiten wegen der hohen Kapazitäten sehr lang. Am anderen Ende des Spektrums liegt die Ökobilanz der Kategorie 3-Wheeler. Die Treibhausgasemissionen sind hier sehr gering, da sowohl das Gewicht als auch der Stromverbrauch sehr niedrig sind. Die Treibhausgasemissionen eines heutigen, elektrisch angetriebenen 3-Wheeler sind dadurch zirka siebenmal niedriger als die eines SUV mit ICE-Motor (siehe Abbildung 33).

Da der Vergleich der Fahrzeugkategorien wenig überraschende Ergebnisse zeigt, werden wir im Folgenden nur noch die Ergebnisse der Kompaktklasse diskutieren. Die vollständigen LCA-Resultate zu allen Fahrzeugklassen finden sich im Anhang 3.

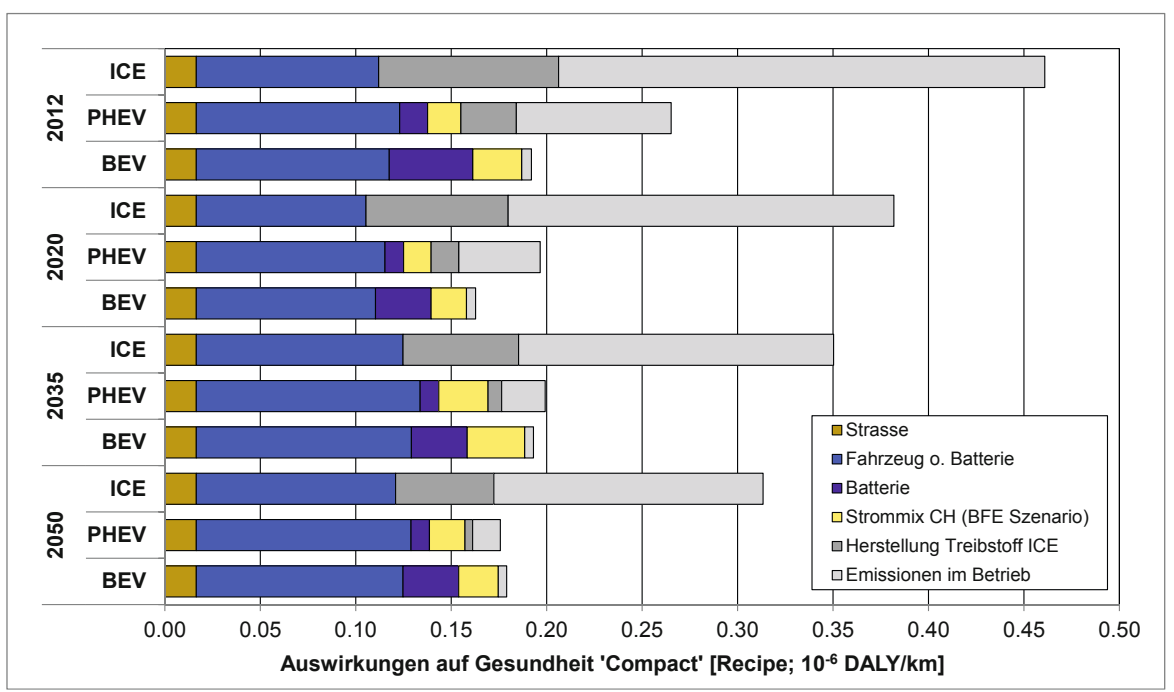

Abbildung 34: Umweltauswirkungen auf die menschliche Gesundheit nach der ReCiPe-Methodik für die drei untersuchten Antriebsstränge (ICE, PHEV, BEV) und die vier Zeitpunkte (2012/20/35/50) für die Fahrzeugklasse «Kompakt». 
Abbildung 34 zeigt die heutigen und zukünftigen Lebenszyklus-Auswirkungen auf die menschliche Gesundheit, berechnet mit der ReCiPe-Methodik (Goedkoop, Heijungs et al. 2009). Die Unterschiede zwischen den einzelnen Fahrzeugen sind geringer als bei den Treibhausgasemissionen. Sie betragen heute rund 30 Prozent zwischen ICE-Fahrzeugen und BEV und nehmen in Zukunft ab auf eine Differenz von etwa 20 Prozent im Jahre 2050. Bei den elektrischen Antrieben ist ein deutlicher Rückgang der Belastung zwischen heute und 2020 zu sehen, der von der grösseren Lebensdauer der Batterie und steigender Energieeffizienz herrührt. Ab 2020 bleiben die Auswirkungen auf die menschliche Gesundheit bei der Elektromobilität weitgehend konstant, da sich Effizienzgewinne und höhere Umweltbelastung in der Stromerzeugung gegenseitig aufwiegen (siehe Abbildung 34).

Hauptgrund für die tendenziell grössere Belastung der menschlichen Gesundheit durch die ICE-Fahrzeuge sind die Fahremissionen, welche bei ICE-Fahrzeugen deutlich höher sind. Es gilt anzumerken, dass der Klimawandel einen beträchtlichen Einfluss auf den Indikator «menschliche Gesundheit» hat, was das grundsätzlich ähnliche Muster wie bei den Treibhausgasemissionen erklärt.

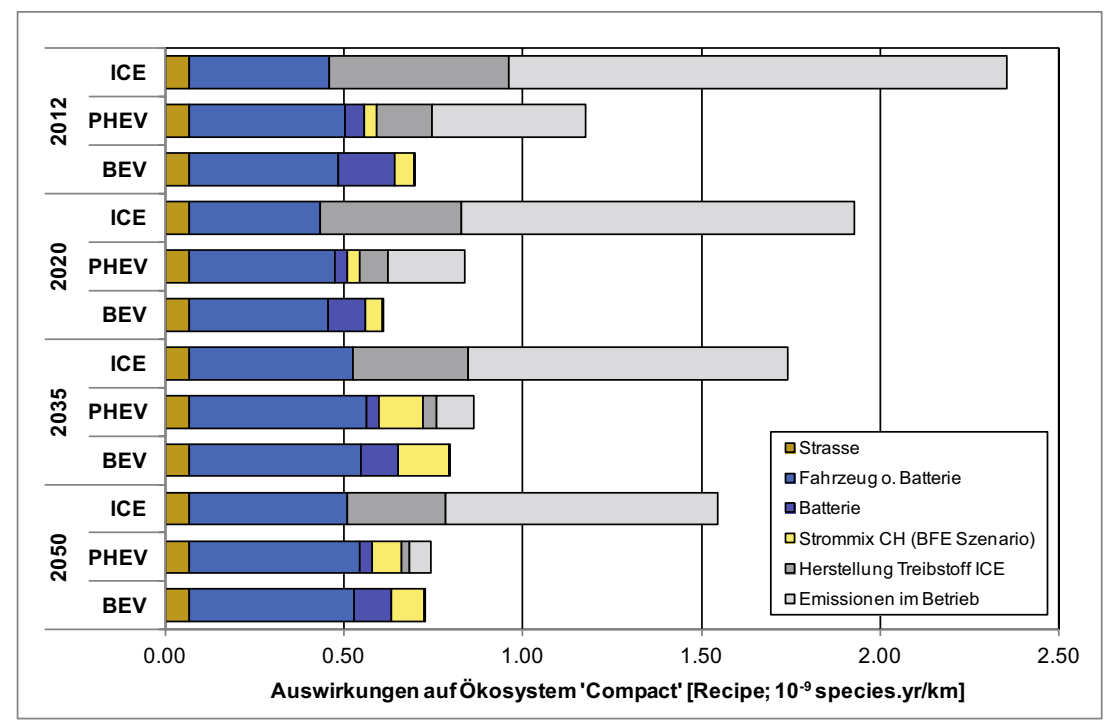

Abbildung 35: Umweltauswirkungen auf Ökosysteme nach der ReCiPe-Methodik für die drei untersuchten Antriebsstränge (ICE, PHEV, BEV) und die vier Zeitpunkte (2012/20/35/50) für die Fahrzeugklasse «Kompakt». 
Die Lebenszyklus-Umweltauswirkungen auf die Ökosysteme sind in Abbildung 35 dargestellt. Das Muster ist sehr ähnlich wie bei den Auswirkungen auf die menschliche Gesundheit. Generell weisen die elektrischen Fahrzeuge 40 bis 50 Prozent weniger Umweltauswirkung auf als die ICE-basierten Fahrzeuge, wobei die Unterschiede in Zukunft kleiner werden (siehe Abbildung 35).

Die Auswirkungen auf die Ressourcenverknappung sind in Abbildung 36 dargestellt. Hier findet logischerweise die Belastung nicht während der Nutzungsphase, sondern ausschliesslich bei der Produktion von Fahrzeug und Treibstoff statt. Da die Verknappung fossiler Treibstoffressourcen in der ReCiPe-Methodik relativ stark gewichtet wird, zeigt sich auch hier ein sehr ähnliches Muster wie bei den vorangegangenen Umweltkategorien. Die Auswirkungen auf die Ressourcenverknappung sind für elektrische Fahrzeuge etwa halb so hoch wie bei den ICE-Fahrzeugen. Die günstige Bewertung der Elektromobilität hängt mit dem fossilarmen Strommix der Schweiz zusammen.

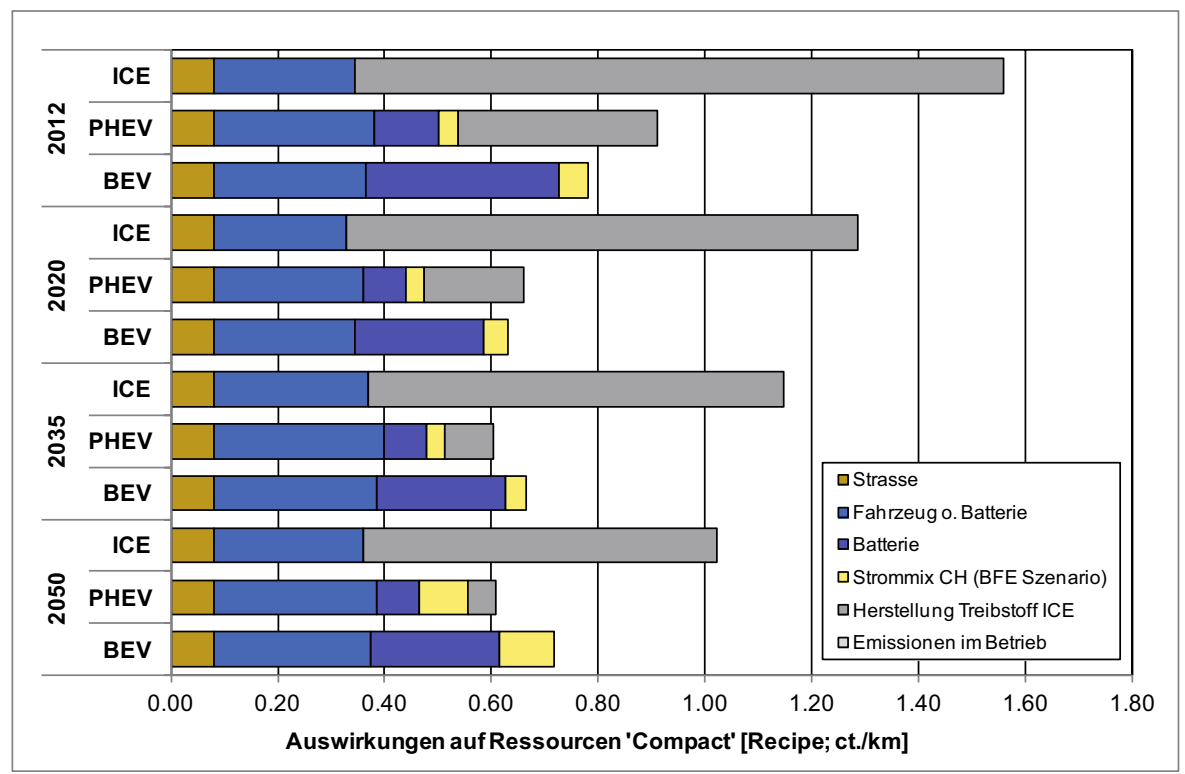

Abbildung 36: Bewertung des Ressourcenverbrauchs nach der ReCiPe-Methodik für die drei untersuchten Antriebsstränge (ICE, PHEV, BEV) und die vier Zeitpunkte (2012/20/35/50) für die Fahrzeugklasse «Kompakt». 


\subsection{Resultate auf der Stufe «Szenarien»}

In Abbildung 37 werden die für die LCA-Rechnung wichtigsten Ergebnisse der Szenarienberechnung zusammengefasst. Links sind die relativen Anteile der Fahrzeugklassen an der Gesamtflotte dargestellt. Beim Business-as-Usual-Szenario (BAU) bleiben die Anteile der Fahrzugklassen über die Jahre konstant. Beim Effizienz-Szenario (EFF) gibt es eine Verschiebung hin zu kleineren Fahrzeugen. Beim Szenario "Connected Mobility» (COM) ist diese Verschiebung noch deutlicher und auch die Fahrzugklasse der 3-Wheeler erlangt einen Marktanteil von 10 Prozent. Aus dieser Flottenzusammensetzung ergeben sich die in Abbildung 37 rechts dargestellten Flottenkilometer. Beim BAU-Szenario ist hierbei der Anteil grosser und mit ICE-Motor gefahrener Kilometer am höchsten (59 Prozent ICE-Fahrten für 2050). Im EFF-Szenario beträgt der Anteil an ICE-Fahrten noch 46 Prozent, im ambitionierten COM-Szenario sind es im Jahr 2050 noch 39 Prozent.

Diese Aufteilung der Flottenkilometer auf verschiedene Fahrzeugklassen und Antriebsstränge bildet nun den Ausgangspunkt zur Berechnung der gesamten Umweltauswirkungen (siehe Abbildung 37).

Abbildung 38 stellt jeweils die insgesamt gefahrenen Flottenkilometer und die dadurch verursachten Treibhausgasemissionen gegenüber. Alle drei Szenarien zeigen massiv rückläufige Trends in den gesamten Treibhausgasemissionen bei einer um über den Zeitraum um 24 Prozent ansteigenden Mobilität. Dieser Rückgang lässt sich einerseits durch die effizienteren ICE-Fahrzeuge im Flottenmix, andererseits aber auch durch Wechsel hin zu Elektrofahrzeugen erklären. Relativ gering sehen jedoch die Unterschiede zwischen den drei Szenarien aus. Während sich für 2020 noch gar kein Unterschied zeigt, beträgt das zusätzliche Sparpotenzial des optimistischen COM-Szenarios gegenüber dem BAUSzenario im Jahr 203517 Prozent und im Jahr 205034 Prozent. 

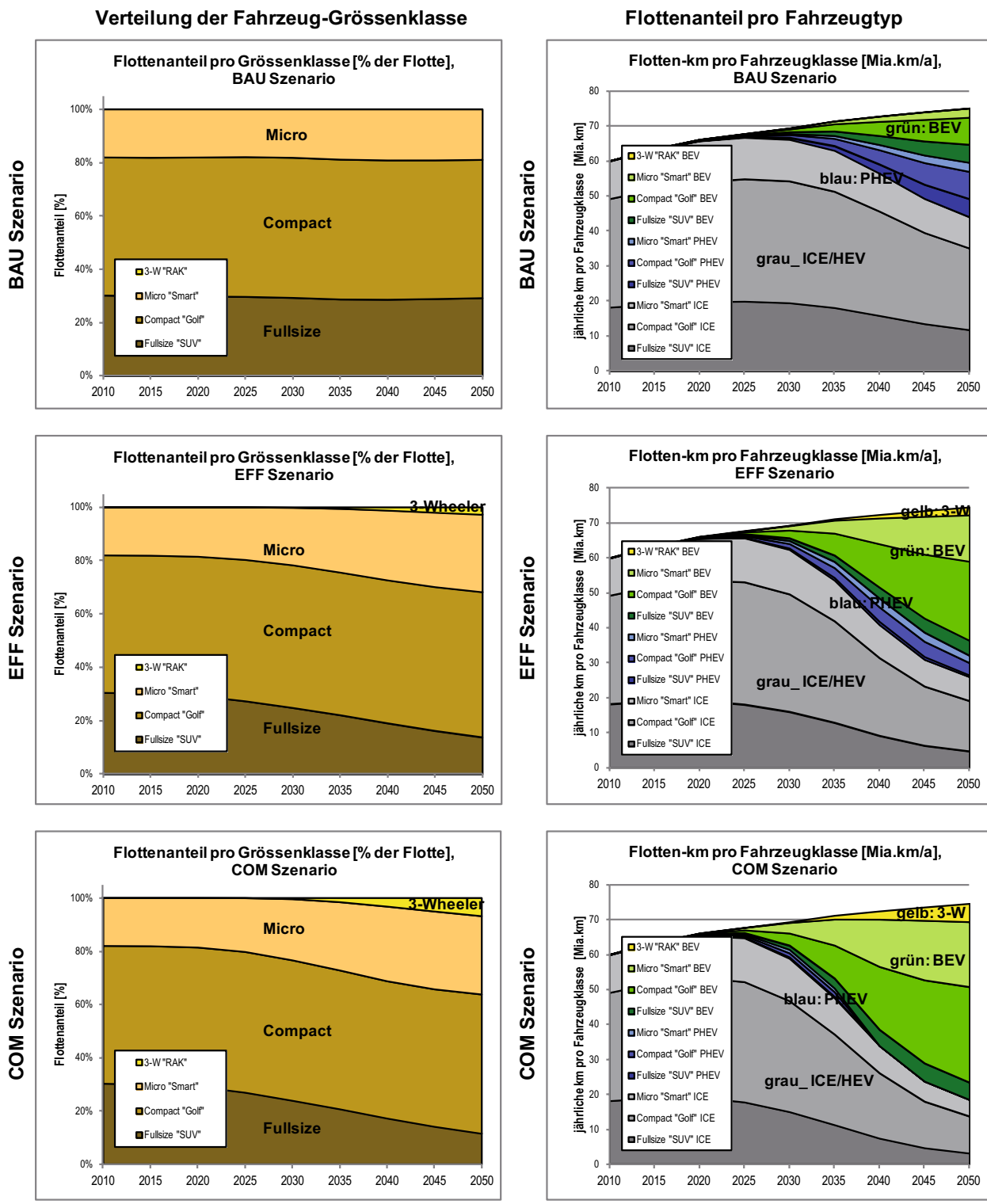

\section{Abbildung 37: Links: relative Anteile der Fahrzeugklassen an der Gesamtflot- te. Rechts: Flottenkilometer der verschiedenen Fahrzeugklas- sen und Antriebstypen.}



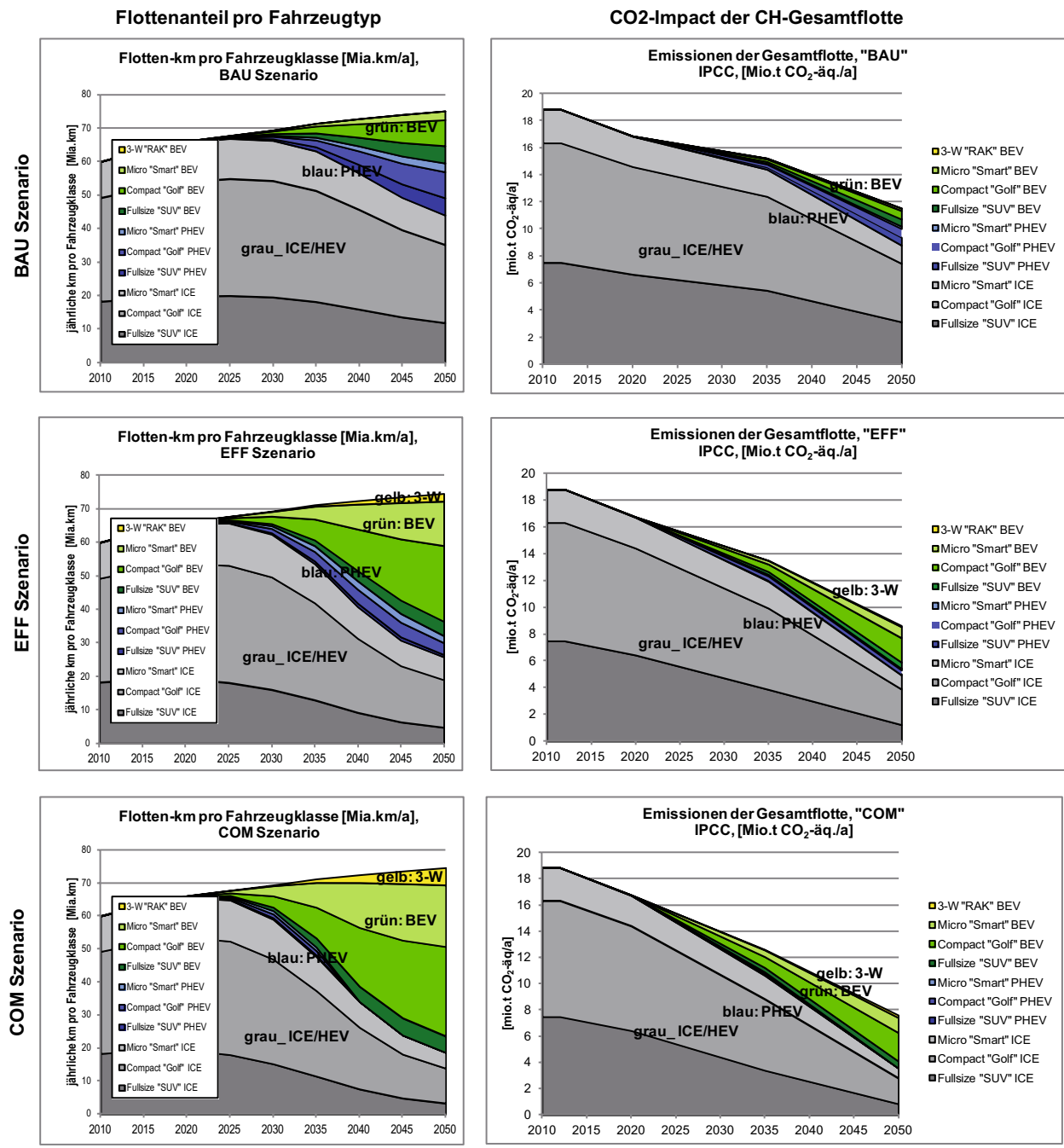

\section{Abbildung 38: Links: Flottenkilometer der verschiedenen Fahrzeugklassen und Antriebstypen. Rechts: Gesamter $\mathrm{CO}_{2}$-Ausstoss der Schweizer Individualflotte.}

In Abbildung 39 fällt auf, dass trotz grossen Fortschritten bei der Effizienz von ICE-Fahrzeugen und der prognostizierten Penetration der Flotte mit Elektrofahr- 
zeugen der Zielpfad für $\mathrm{CO}_{2}$-Reduktionen für die NEFZ-Betriebsemissionen nicht eingehalten werden kann. Dazu muss jedoch gesagt werden, dass die EUPrognosen von null Emissionen der reinen Elektrofahrzeuge ausgehen. In der Studie wurden die Emissionen der Bereitstellung der Elektrizität berücksichtigt, weshalb die Resultate höher erscheinen.

Es fällt ebenfalls auf, dass die NEFZ-Emissionen weniger als die Hälfte der Emissionen bei einer vollen Lebenszyklusanalyse ausmachen. Die in Prognosen meist übliche Vernachlässigung des Aufwands für den Bau der Fahrzeuge führt also zu einer deutlichen Unterschätzung der wahren Emissionen.

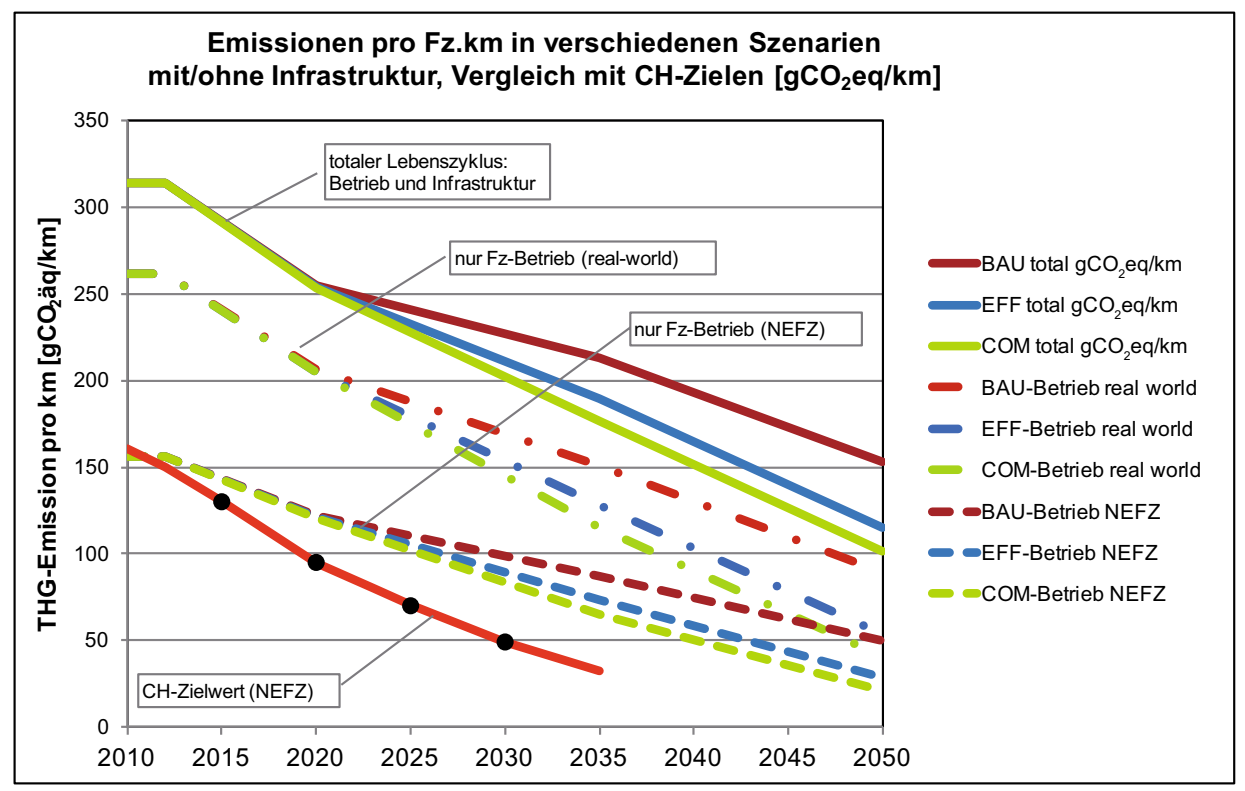

Abbildung 39: Entwicklung des gesamten $\mathrm{CO}_{2}$-Ausstosses der Schweizer Individualflotte in den drei Szenarien verglichen mit dem angestrebten EU-Reduktionspfad. Punktlinie: Reine NEFZEmissionen im Betrieb. Gestrichelte Linie: Real-World-Emissionen im Betrieb. Ausgezogene Linie: Gesamte Auswirkung inkl. Infrastrukturaufwand zum Bau und Betrieb der Fahrzeuge. 


\subsection{Unsicherheiten der Lebenszyklusanalye}

\subsubsection{Sensitivitätsanalyse der Annahmen zu den Fahrzeugwirkungs- graden}

Die Abschätzung der zukünftigen Technologieentwicklung der Elektromobilität ist mit Unsicherheiten verbunden. Besonders für den Zeitraum nach 2030 sind diese gross, da die Technologieentwicklung stark vom Eintreffen oder Ausbleiben von Entwicklungssprüngen abhängt, wie beispielsweise der Markteinführung neuer Elektrodentechnologien bei Li-lon-Batterien. Um die Auswirkungen dieser Unsicherheiten auf die Ökobilanz aufzuzeigen, wurde eine Sensitivitätsanalyse durchgeführt. Hierbei wurden sowohl für Fahrzeugparameter (Gewicht, Rollwiderstand etc.) als auch für Parameter des Antriebsstrangs (Wirkungsgrad von Antrieb und Ladegerät) pessimistische und optimistische Annahmen getroffen und jeweils zu einem Worst-Case- und einem Best-Case-Szenario kombiniert. Zusammenfassend wurden die folgenden Annahmen getroffen (Details siehe Tabelle im Anhang):

- BEV worst: Keine Fortschritte angenommen (Technologiestand von 2012 bleibt konstant bis 2050) und hoher Energiebedarf (NEFZ +40 Prozent), Widerstandsheizung; gleiches Fahrzeuggewicht trotz Aluminium (mehr Komfortkomponenten).

- BEV best: Deutliche Fortschritte bei Luft- und Rollwiderstand, 1 Prozent Gewichtsreduktion pro Jahr. Energiebedarf wie NEFZ +10 Prozent Zuschlag, Wärmepumpe.

Die Ergebnisse der Sensitivitätsanalyse sind in Abbildung 40 dargestellt. Aufgrund des in der Schweiz sehr $\mathrm{CO}_{2}$-armen Stroms wirkt sich die stark reduzierte Effizienz der Elektrofahrzeuge nur relativ schwach auf die gesamte $\mathrm{CO}_{2}$-Bilanz aus (gelbe Balken). Auch unter Worst-Case-Effizienz-Annahmen ist die Treibhausgasbilanz der Elektrofahrzeuge daher noch deutlich besser als die fossile Referenz. Anders können die Verhältnisse in Regionen mit einem höheren Anteil an fossil erzeugtem Strom (z.B. in Deutschland) aussehen. Hierzu sei auf die Sensitivitätsanalyse zur Stromanalyse verwiesen (Kapitel 5.6.2).

Ebenso stark wirken sich die Unsicherheiten bei der Entwicklung des Fahrzeuggewichts und damit des Produktionsaufwands der Fahrzeuge auf die Treibhausgasbilanz aus (blaue Balken). 
Generell zeigt die Sensitivitätsanalyse, dass die Entwicklung der Fahrzeugeffizienz nur eine relativ kleine Auswirkung auf die gesamte Treibhausgasbilanz hat und der generelle Trend sehr stabil ist.

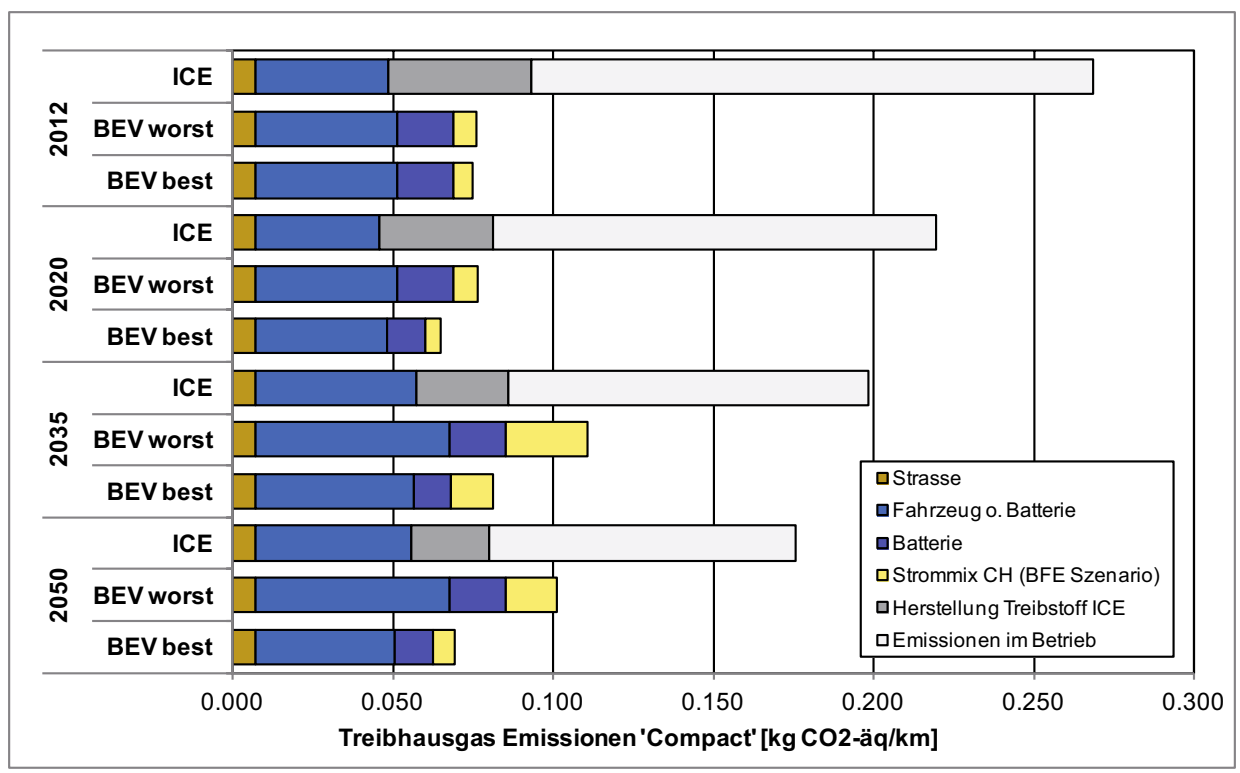

Abbildung 40: Auswirkungen von Best- und Worst-Case-Annahmen zur Fahrzeugentwicklung von Elektromobilen beim Schweizer Strommix auf die Treibhausgasemissionen im gesamten Lebenszyklus.

\subsubsection{Sensitivitätsanalyse zur Stromerzeugung}

Die Abbildung 41 zeigt, wie sich die Treibhausgasemissionen der betrachteten Fahrzeuge verändern, wenn für die Nutzung der Fahrzeuge die Art der Stromerzeugung variiert wird. Als Best Case wurde zertifizierter Schweizer Ökostrom aus Wasserkraft und Fotovoltaik angenommen, als Worst Case der europäische UCTE-Strommix herangezogen, der zu 52 Prozent fossile Stromerzeugung beinhaltet. Da sich auch die Produktion fossiler Treibstoffe ändern kann, wurde hierzu als Worst Case Treibstoff aus kanadischen Ölsanden mitbetrachtet. 


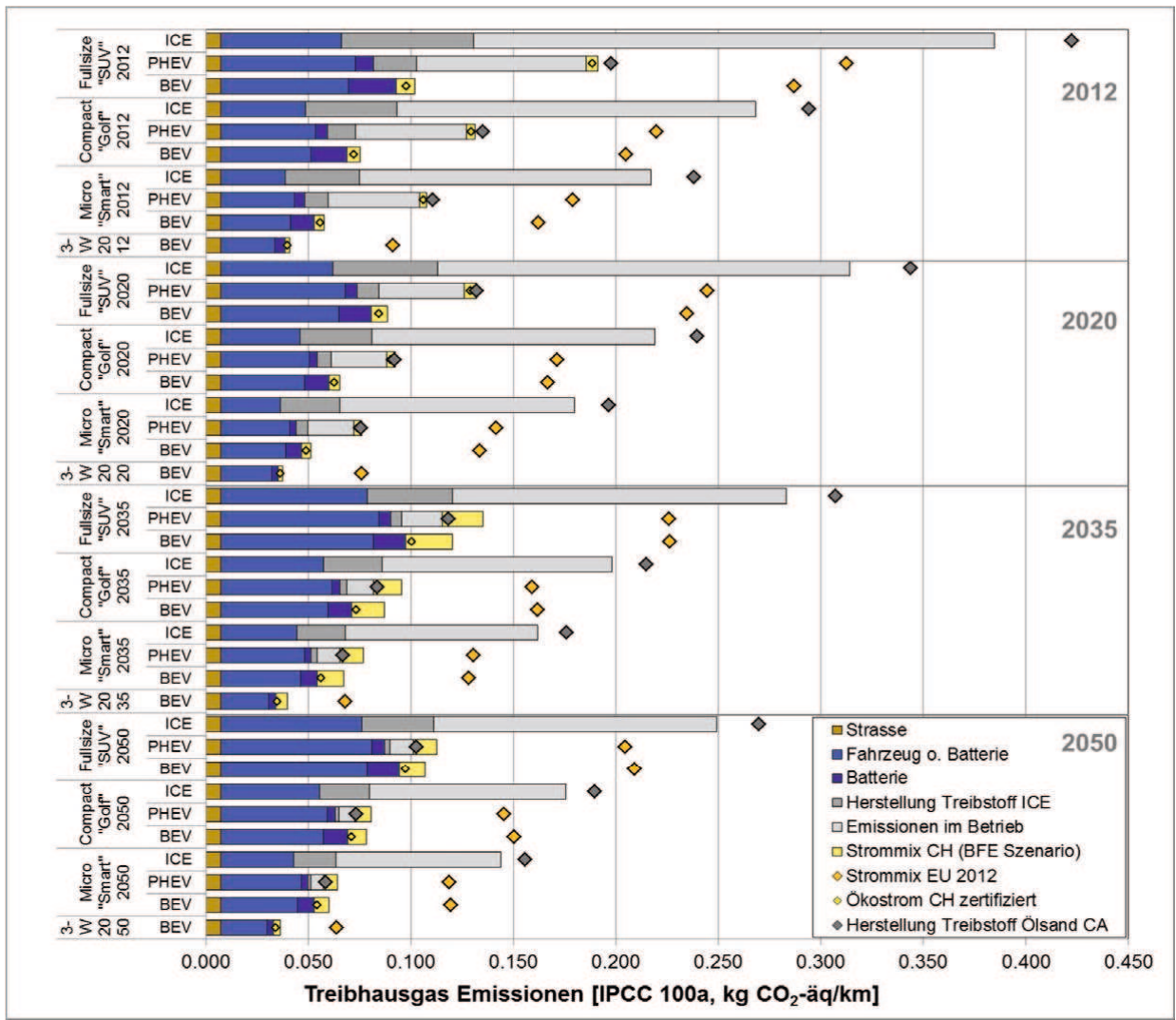

Abbildung 41: Lebenszyklus-Treibhausgasemissionen für die drei untersuchten Antriebsstränge (ICE, PHEV, BEV) und die vier Zeitpunkte (2012/20/35/50) für die vier Fahrzeugklassen (Fullsize, Compact, Micro, 3-Wheeler). Die Punkte bezeichnen jeweils die Veränderung bei Anpassung der Benzinqualität (bei ICE, Benzin aus kanadischen Ölsanden) und bei Veränderung des Strommixes für PHEV/BEV-Fahrzeuge (europäischer Strommix bzw. zertifizierter $\mathrm{CH}$-Ökostrom). 
Die Ergebnisse bestätigen die hohe Sensitivität gegenüber der Stromerzeugung. Während die $\mathrm{CO}_{2}$-Einsparung eines $\mathrm{BEV}$ der Kompaktklasse gegenüber dem ICE-Fahrzeug heute 70 Prozent beträgt, reduziert sich die Einsparung auf knapp 20 Prozent, wenn UCTE-Strom getankt wird. Bei reinem Kohlestrom wäre die Treibhausgasbelastung des BEV sogar noch höher. In der Zukunft werden die negativen Auswirkungen des UCTE-Strommixes auf die $\mathrm{CO}_{2}$-Bilanz im Vergleich zu den immer effizienteren ICE-Fahrzeugen noch grösser: 2050 würde ein mit (dem heutigen) UCTE-Mix getanktes BEV gegenüber einem ICE-Fahrzeug keine $\mathrm{CO}_{2}$-Einsparung mehr erzielen.

Deutlich geringer ist dagegen der negative Effekt unkonventioneller Ölproduktion auf die ICE-Treibhausgasemissionen. Im Schnitt führt dies zu zirka 10 Prozent zusätzlichen Treibhausgasemissionen.

Diese Interpretationen gelten nur unter Annahme einer konstant hoch bleibenden $\mathrm{CO}_{2}$-Belastung des europäischen Durchschnittsstrommixes und unter der Annahme, dass der Aufwand zur Gewinnung und Raffinierung von fossilem Treibstoff im Jahr 2050 gleich hoch sein wird wie heute.

Auf globaler Stufe zeigt eine Analyse der Strommarktprognosen der internationalen Energieagentur IEA (IEA 2011, siehe Abbildung 58), dass sich die $\mathrm{CO}_{2}-$ Belastung des globalen Strommixes auf ähnlich hohem Niveau wie bei dem europäischen Strommix bewegt (ca. $600 \mathrm{~g} \mathrm{CO}$ eq/kWh). Die Belastung pro Kilowattstunde geht jedoch trotz Zubau von Kohlekraftwerken im Lauf der Zeit zurück, weil zunehmend in erneuerbare Energien investiert wird und die neu dazukommenden fossilen Kraftwerke effizienter als die existierenden Kraftwerke sind. 


\section{$6 \quad$ Mobilität und kritische Rohstoffe}

\subsection{Kritikalität metallischer Rohstoffe}

In den vergangenen fünf Jahren ist eine Reihe von Studien erschienen, die sich mit den Versorgungsrisiken von metallischen Rohstoffen, speziell seltenen Metallen ${ }^{2}$, und ihren möglichen Auswirkungen befasst haben (European Commission 2010; National Research Council 2008; U.S. DOE 2011). Zur ihrer Beurteilung wird dabei häufig das Konzept der Kritikalität angewendet, das vom U.S. National Research Council (NRC) eingeführt und anhand einer zweidimensionalen Kritikalitätsmatrix illustriert worden ist (Abbildung 42).

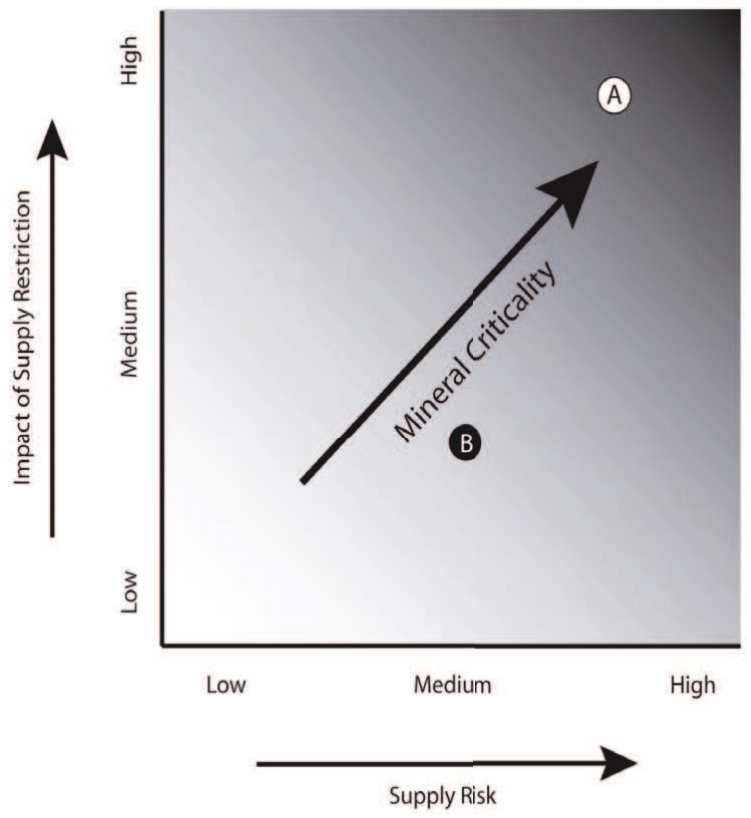

Abbildung 42: Kritikalitätsmatrix gemäss National Research Council (National Research Council 2008).

2 Konzentrationen < 0.01 Gewichtsprozent vorkommen (Wäger et al. 2010). 
Innerhalb der Kritikalitätsmatrix nimmt die Kritikalität von der unteren linken Ecke zur oberen rechten Ecke der Matrix zu, wobei die horizontale Achse die Wahrscheinlichkeit einer Versorgungsbeschränkung eines Rohstoffes und die vertikale Achse die Auswirkungen dieser Beschränkung darstellen.

Kritikalität ist ein relatives Konzept: Gemäss der Definition der Ad-hoc-Arbeitsgruppe zur Definition kritischer Rohstoffe der Europäischen Kommission wird ein Material als kritisch bezeichnet, wenn das Versorgungsrisiko und die potenziellen Auswirkungen eines Versorgungsunterbruchs höher sind als für die meisten anderen Rohstoffe (European Commission 2010).

Bei der Anwendung des Konzeptes der Kritikalität sind verschiedene Einschränkungen zu berücksichtigen.

Die Beurteilung der Kritikalität beruht auf einer Vorauswahl von Rohstoffen, die potenziell kritisch sein könnten (Erdmann und Graedel 2011). In der Studie des NRC wurden beispielsweise 11 mineralische Rohstoffe untersucht, während es in der Ad-hoc-Studie der Europäischen Kommission deren 41 waren.

Die Beurteilung der Kritikalität erfolgt immer aus einer ganz bestimmten Perspektive wie beispielsweise Unternehmen, Sektor, Land oder globale Gesellschaft. Aufgrund der unterschiedlichen Versorgungsrisiken sowie Auswirkungen von Versorgungsunterbrüchen kann dies auch bei gleichem Vorgehen zu unterschiedlichen Ergebnissen führen (Erdmann und Graedel 2011).

Das Ergebnis der Beurteilung der Kritikalität entspricht einer Momentaufnahme. Deshalb empfiehlt die Ad-hoc-Arbeitsgruppe, die Beurteilung regelmässig zu wiederholen (European Commission 2010).

Folgende Stoffe werden in bestehenden Kritikalitätsstudien ${ }^{3}$ am häufigsten als kritisch beurteilt: Indium, Niob, Platin, Rhodium, Ruthen, Seltenerdelemente und Wolfram. Weitere, häufig als kritisch beurteilte Rohstoffe sind: Antimon, Beryllium, Kobalt, Fluor, Gallium, Germanium, Lithium, Magnesium, die verbleibenden Platinmetale (Iridium, Osmium, Palladium), Zinn und Zirkon (Erdmann und Graedel 2011). ley, N. (2008), Pfleger et al. (2009), Shinko Research (Mitsubishi UFJ Research and Consulting) (2009), Thomason et al. (2008). 
In der vorliegenden Studie stecken diese Rohstoffe den Untersuchungsrahmen ab, ergänzt durch die Edelmetalle Gold und Silber, welche in Elektro- und Elektronikgeräten eine wichtige Rolle spielen. Als Hauptursachen für die Kritikalität dieser Materialien wurde die Konzentration des Angebots auf wenige Länder identifiziert, verbunden mit fehlenden Substitutionsmöglichkeiten und geringen Recyclingraten (European Commission 2010). Im Gegensatz etwa zur Studie des National Research Council wurde die geologische Verfügbarkeit der Rohstoffe in dieser Studie nicht berücksichtigt, weil die verfügbaren Angaben zu den globalen Reserven ${ }^{4}$, Grenzreserven ${ }^{5}$ und Ressourcen ${ }^{6}$ und die daraus berechneten statischen Reichweiten ${ }^{7}$ als dafür nicht geeignet beurteilt wurden. Dies unter anderem, weil Bergbauunternehmen erst bei anstehenden Investitionen in die Anpassung der Zahlen für die Reserven investieren und die Reserven in den vergangenen 50 Jahren immer wieder aufgefüllt werden konnten.

Der Abbau und die Raffination von metallischen Rohstoffen sind energieintensiv und mit Umweltauswirkungen verbunden. Die Umweltauswirkungen sind besonders hoch für Edelmetalle wie Palladium, Rhodium, Platin oder Gold. Unter Berücksichtigung der Jahresproduktionen gehen allerdings bei den metallischen Rohstoffen nach wie vor die höchsten Umweltauswirkungen von traditionellen Industriemetallen wie Eisen, Chrom, Aluminium, Nickel und Kupfer aus (UNEP 2010, Wäger 2011).

Der Umweltdimension wurde in den bisherigen Kritikalitätsstudien, wenn überhaupt, dann meist nur implizit Beachtung geschenkt. Die Ad-hoc-Gruppe der Europäischen Kommission hat mit dem Indikator «Environmental Country Risk» das Risiko für die länderspezifische Einführung von Umweltmassnahmen quantifiziert, weil diese den Zugang zu Rohstofflagerstätten und die Versorgung mit Rohstoffen einschränken könnten (European Commission 2010). Kürzlich wurde

Reserven beinhalten denjenigen Teil der Reservebasis, der unter den gegenwärtigen ökonomischen und technischen Rahmenbedingungen gefördert werden kann. Reserven sind als provisorische Inventare zu betrachten, die kontinuierlich revidiert werden (European Commission 2010; USGS 2012).

5 Die Reservebasis bzw. die Grenzreserven umfassen denjenigen Teil der Ressourcen, der spezifische, auf gängige Bergbau- und Produktionspraktiken bezogene physikalische und chemische Mindestanforderungen erfüllt, einschliesslich u.a. Erzgehalt und Abbautiefe (USGS 2012).

6 Der Begriff der Ressource bezeichnet eine Ansammlung natürlich auftretender, gasförmiger, flüssiger oder fester Materialien in oder auf der Erdkruste, aus der ein Rohstoff gegenwärtig oder möglicherweise in Zukunft ökonomisch gewonnen werden kann (USGS 2012).

$7 \quad$ Das Verhältnis zwischen den weltweiten Reserven oder Grenzreserven und der Weltjahresproduktion eines Metalls. 
eine Weiterentwicklung der vom National Research Council angewendeten Methodik veröffentlicht, in der die aus den zwei ursprünglichen Dimensionen (Versorgungsrisiko und Anfälligkeit auf Versorgungsunterbrüche) bestehende Kritikalitätsmatrix durch die Dimension «Umweltimplikationen» zu einem «Kritikalitätsraum» ergänzt wurde (Graedel et al. 2011; Nassar et al. 2011).

\subsection{Kritische metallische Rohstoffe in den unter- suchten Fahrzeugtypen}

Tabelle 20 gibt eine Übersicht über die in Komponenten der untersuchten Fahrzeugtypen (EV, HEV, PEV, ICEV) enthaltenen kritischen Metalle. Die Tabelle beruht auf ersten Abklärungen im Rahmen eines kürzlich angelaufenen Projektes (Blaser et al. 2012); andere öffentlich zugängliche Daten lagen nicht vor. Wie daraus hervorgeht, lassen sich anzahlmässig die meisten kritischeren seltenen Metalle in der in Fahrzeugen eingebauten Informations- und Kommunikationstechnik sowie in der Steuertechnik finden. Seltenerdelemente, Zinn und Kobalt sind diejenigen seltenen Metalle, die in den meisten Komponenten anzutreffen sind. Besonders hohe Konzentrationen an kritischen Metallen werden insbesondere in Leiterplatten (alle), Traktionsbatterien (Lithium, Seltenerdelemente), Elektromotoren (Neodym) und Katalysatoren (PMG) erwartet (siehe Tabelle 20).

In den folgenden Abschnitten werden exemplarisch drei seltene Metalle oder Metallgruppen hinsichtlich erwarteter Entwicklungen bei Nachfrage und Versorgung in Zusammenhang mit der zukünftigen Mobilität untersucht:

- Lithium als wesentlicher Bestandteil von Traktionsbatterien für Elektrofahrzeuge;

- Seltenerdelemente als wichtige Rohstoffe für leistungsfähige Permanentmagnete in Elektromotoren;

- Platinmetalle als Katalysatoren zur Reduktion des Schadstoffausstosses aus Verbrennungsmotoren. 


\begin{tabular}{|c|c|c|c|c|c|c|c|c|c|c|c|c|c|c|c|c|c|}
\hline & Komponenten & $\begin{array}{l}\bar{\Phi} \\
\stackrel{\stackrel{0}{\bar{\omega}}}{\bar{\omega}}\end{array}$ & $\begin{array}{l}\text { 응 } \\
0\end{array}$ & 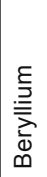 & $\begin{array}{l}\frac{\pi}{\pi} \\
\frac{0}{0} \\
\underline{2}\end{array}$ & 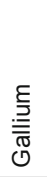 & 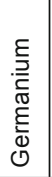 & $\begin{array}{l}\underline{\underline{\underline{g}}} \\
\underline{\underline{z}} \\
\underline{\underline{\Xi}}\end{array}$ & 鸹 & 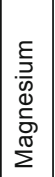 & $\stackrel{\circ}{\circ}$ & 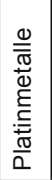 & 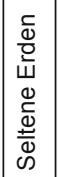 & 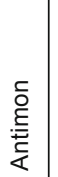 & $\stackrel{\complement}{\stackrel{ \pm}{N}}$ & 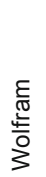 & 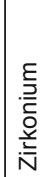 \\
\hline $\begin{array}{l}\text { BEV, HEV, } \\
\text { PHEV, ICE }\end{array}$ & Elektromotoren & - & - & - & $\mathrm{x}$ & - & - & - & - & - & - & - & $x$ & - & $\mathrm{x}$ & - & - \\
\hline $\begin{array}{l}\text { BEV, HEV, } \\
\text { PHEV, ICE }\end{array}$ & IKT/UE & $x$ & $x$ & $x$ & $x$ & $\mathrm{x}$ & $x$ & $\mathrm{x}$ & - & - & $\mathrm{x}$ & $x$ & $\mathrm{x}$ & $\mathrm{x}$ & $\mathrm{x}$ & $\mathrm{x}$ & $\mathrm{x}$ \\
\hline $\begin{array}{l}\text { BEV, HEV, } \\
\text { PHEV, ICE }\end{array}$ & Kontakte & - & $\mathrm{x}$ & $\mathrm{x}$ & - & - & - & - & - & - & - & - & - & - & $\mathrm{x}$ & - & - \\
\hline $\begin{array}{l}\text { BEV, HEV, } \\
\text { PHEV, ICE }\end{array}$ & Sensoren & - & - & - & - & - & - & - & - & - & - & $?$ & $?$ & - & $\mathrm{x}$ & - & $x$ \\
\hline $\begin{array}{l}\text { BEV, HEV, } \\
\text { PHEV, ICE }\end{array}$ & Steuergeräte & $\mathrm{x}$ & $\mathrm{x}$ & $x$ & $x$ & $\mathrm{x}$ & $\mathrm{x}$ & $\mathrm{x}$ & - & - & $\mathrm{x}$ & $\mathrm{x}$ & $\mathrm{x}$ & $\mathrm{x}$ & $\mathrm{x}$ & $x$ & $x$ \\
\hline $\begin{array}{l}\text { BEV, HEV, } \\
\text { PHEV, ICE }\end{array}$ & $\begin{array}{l}\text { User Interface } \\
\text { (Cockpit) }\end{array}$ & $x$ & $\mathrm{x}$ & - & - & $x$ & - & $x$ & - & - & - & - & $x$ & - & $\mathrm{x}$ & $?$ & - \\
\hline $\begin{array}{l}\text { BEV, HEV, } \\
\text { PHEV, ICE }\end{array}$ & Aktoren & - & - & - & $?$ & - & - & - & - & - & - & - & $?$ & - & $\mathrm{x}$ & - & - \\
\hline $\begin{array}{l}\text { BEV, HEV, } \\
\text { PHEV, ICE }\end{array}$ & Beleuchtung & - & - & - & - & $\mathrm{x}$ & - & $\mathrm{x}$ & - & - & - & - & $\mathrm{x}$ & - & $x$ & $x$ & - \\
\hline $\begin{array}{l}\text { BEV, HEV, } \\
\text { PHEV, ICE }\end{array}$ & Batterie & - & - & - & $x$ & - & - & - & $\mathrm{x}$ & - & - & - & $\mathrm{x}$ & - & - & - & - \\
\hline $\begin{array}{l}\text { HEV; } \\
\text { PHEV, ICE }\end{array}$ & Katalysatoren & - & - & - & - & - & - & - & - & - & - & $\mathrm{x}$ & $\mathrm{x}$ & - & - & - & - \\
\hline $\begin{array}{l}\text { BEV; HEV; } \\
\text { HEV; ICE }\end{array}$ & Antriebsstrang & - & - & - & $x$ & - & - & - & - & $\mathrm{x}$ & $x$ & - & $?$ & $?$ & - & $x$ & - \\
\hline $\begin{array}{l}\text { BEV; HEV; } \\
\text { HEV; ICE }\end{array}$ & Glider & - & - & - & $x$ & - & - & - & - & $x$ & $x$ & - & $?$ & $\mathrm{x}$ & $?$ & - & - \\
\hline
\end{tabular}

Tabelle 20: Vorkommen kritischer Metalle in ausgewählten Komponenten heutiger und zukünftiger Fahrzeuge:

$\mathrm{x} \quad$ kommt mit grosser Wahrscheinlichkeit vor;

? kommt eventuell vor;

- $\quad$ kommt eher nicht vor.

\subsection{Fokus Lithium}

\subsubsection{Anwendungen}

Lithium wird unter anderem in der Glas- und Keramikindustrie oder in Schmierfetten verwendet. Von immer grösserer Bedeutung ist Lithium als Rohstoff für die Herstellung von Sekundärbatterien für mobile elektronische und elektrische Ge- 
räte. Dies beinhaltet sowohl kleinere Batterien für elektronische Geräte wie beispielsweise Laptops, Mobiltelefone oder Navigationssysteme als auch grosse Batterien für Elektro- und Hybridfahrzeuge (Angerer et al. 2009b). Zwischen 1980 und 2009 hat sich die Nachfrage nach Lithium insgesamt verdreifacht; im Jahr 2010 lag sie bei 150 Kilotonnen. Die verstärkte Nachfrage nach Lithium für die Produktion von aufladbaren Batterien führte ab 2005 zu einem starken Anstieg des davor stabilen Preises von 4000 USD/kg auf 6000 USD/kg im Jahr 2009. Danach sank der Preis jedoch wieder auf rund 4500 USD/kg im Jahr 2010 aufgrund von Preisreduktionen von verschiedenen Lithiumherstellern (U.S. DOE 2011).

\subsubsection{Globale Nachfrage}

Für die nächsten Jahre wird ein weiterer Anstieg des Lithiumverbrauchs erwartet, welcher wesentlich durch die Entwicklungen im Bereich der Elektro- und Hybridfahrzeuge getrieben sein wird (Angerer et al. 2009b). Dies insbesondere deshalb, weil Li-Traktionsbatterien eine vergleichsweise hohe Menge an Lithium benötigen und weil erwartet wird, dass die Anzahl Fahrzeuge, welche künftig mit Lithium-Technologie betrieben werden könnten, sehr stark ansteigen wird (Angerer et al. 2009b).

Gemäss Szenarien des U.S. DOE (2011) könnte der Anteil der neuen Energien sowie der Elektromobilität an der Lithium-Nachfrage von einem Prozent im Jahr 2010 auf über 50 Prozent im Jahr 2025 ansteigen. Die jährliche Nachfrage 2025 würde dabei zwischen 200 und 900 Kilotonnen Lithium liegen.

Aus heute genutzten Lithiumquellen lassen sich rund 250 Kilotonnen Lithium pro Jahr produzieren, womit die kurzfristige Lithiumnachfrage gedeckt werden könnte (Abbildung 43). Für Szenarien mit einer hohen weltweiten Marktpenetration durch Elektrofahrzeuge wird jedoch das bestehende Angebot langfristig nicht zur Deckung der Nachfrage ausreichen und erfordert die Erschliessung neuer Reserven (U.S. DOE 2011). Dies führt mittel- bis langfristig zu einem höheren materiellen und energetischen Aufwand bei der Rohstoffgewinnung, mit entsprechenden negativen Auswirkungen auf Umwelt und Gesellschaft (Carles 2010; Stamp et al. 2012). 


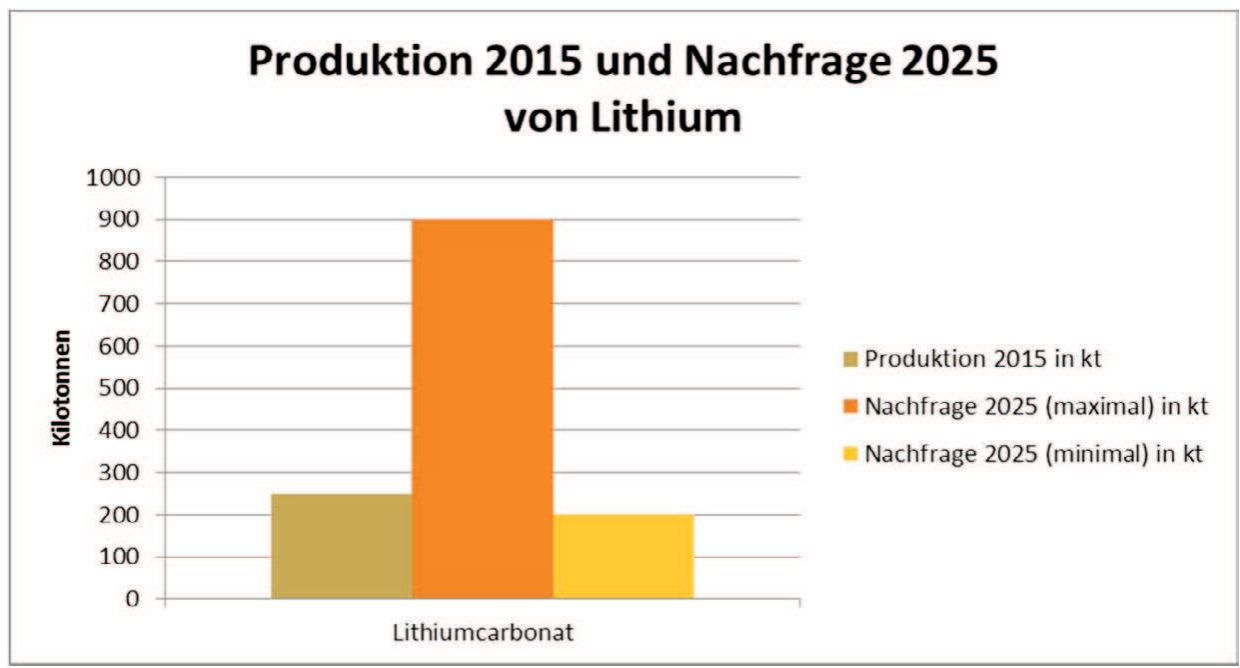

Abbildung 43: Gegenüberstellung von Produktionsniveau 2015 und minimale sowie maximale Nachfrage 2025 für Lithiumcarbonat $\left(\mathrm{Li}_{2} \mathrm{CO}_{3}\right)$ (U.S. DOE 2011).

\subsubsection{Nachfrage durch Neuwagen in der Schweiz}

Für eine Abschätzung der Nachfrage nach Lithium in den in der vorliegenden Studie betrachteten Szenarien wurde davon ausgegangen, dass die Traktionsbatterien die Hauptverbraucher von Lithium darstellen. In einem ersten Schritt wurde deshalb der Lithiumbedarf pro Batteriekapazitätseinheit ermittelt. Unter der Annahme, dass der Energieträger Lithium über den gesamten betrachteten Zeitraum eine konstante Energiespeicherkapazität haben wird, ergibt dies konstante 0,1 Kilogramm Lithium pro Kilowattstunde (Notter et al., 2010). Mithilfe der im Modell für die Szenarioberechnungen enthaltenen Batteriekapazitäten pro Fahrzeugkategorie wurde anschliessend der jeweilige Lithiumbedarf pro Fahrzeugkategorie berechnet (siehe Tabelle 21) und diese wiederum mit den Fahrzeugverkäufen gemäss den drei Szenarien verknüpft ${ }^{8}$. Aus den resultierenden jährlichen Lithiumzuströmen wurden die kumulierten Lithiumrohstofflager abgeleitet. gung bzw. das Recycling) wurden nicht berücksichtigt. 


\begin{tabular}{|l|c|c|c|c|c|}
\hline \multirow{4}{*}{ Fullsize SUV } & & $\mathbf{2 0 1 2}$ & $\mathbf{2 0 2 0}$ & $\mathbf{2 0 3 5}$ & $\mathbf{2 0 5 0}$ \\
\cline { 2 - 6 } & ICE & - & - & - & - \\
\cline { 2 - 6 } & PHEV & 1,5 & 2,3 & 4,7 & 5,4 \\
\cline { 2 - 6 } & BEV & 4,0 & 6,2 & 12,5 & 14,4 \\
\hline \multirow{4}{*}{ Compact Golf } & ICE & - & - & - & - \\
\cline { 2 - 6 } & PHEV & 1,0 & 1,5 & 3,1 & 3,6 \\
\cline { 2 - 6 } & BEV & 3,0 & 4,6 & 9,4 & 10,8 \\
\hline \multirow{3}{*}{ Micro Smart } & ICE & - & - & - & - \\
\cline { 2 - 6 } & PHEV & 0,8 & 1,2 & 2,5 & 2,9 \\
\cline { 2 - 6 } & BEV & 2,0 & 3,1 & 6,3 & 7,2 \\
\hline 3-Wheeler RAKe & BEV & 0,8 & 1,2 & 2,5 & 2,9 \\
\hline
\end{tabular}

Tabelle 21: $\quad$ Lithiumbedarf pro Fahrzeug (in kg).

In Abbildung 44 sind der jährliche Lithiumzustrom durch Neuwagen sowie das Wachstum des entsprechenden Lithiumrohstofflagers für jedes der drei Szenarien dargestellt. Wie daraus hervorgeht, steigt der jährliche Lithiumzustrom durch Neuwagen von knapp unter einer Tonne im Jahr 2015 auf Werte zwischen rund 1640 t/a und 1685 t/a im Jahr 2050 an. Das resultierende Lithiumrohstofflager im Jahr 2050 beträgt zwischen rund 22100 Tonnen und 30900 Tonnen (siehe Abbildung 44).

\subsubsection{Mögliche Massnahmen}

Massnahmen, die eine Erschliessung neuer Lithiumreserven begrenzen bzw. ganz überflüssig machen könnten, wären ein verstärktes Recycling bzw. eine Substitution. Eine Rückgewinnung von Lithium aus Lithiumbatterien findet derzeit noch kaum statt. Gesetzliche Vorgaben, wie sie in der europäischen Batterierichtlinie 2006/66/EC, der WEEE-Richtlinie 2002/96/EC und nationalen Rücknahme- und Verwertungsregeln zu finden sind, werden allerdings mittelfristig zum Ausbau des Recyclings führen. So sollen 2012 12,5 Prozent und bis 2016 22,5 Prozent der verkauften Lithium-Batterien recykliert werden, wobei allerdings vermutlich zunächst eher die teureren metallischen Anteile der Batterie (Kobalt, Kupfer) zurückgewonnen werden. Insgesamt wird das Recycling auch in Zukunft gegenüber der Primärproduktion eine untergeordnete Rolle spielen, wenn sich die Nachfrage so entwickelt wie z.B. von U.S. DOE (2011) erwartet (Carles 2010). Eine Möglichkeit der Substitution könnte beispielsweise darin bestehen, LithiumAkkumulatoren durch solche zu ersetzen, die auf häufiger vorkommenden Erdalkalimetallen aufbauen wie zum Beispiel Natrium-Schwefelbatterien. 

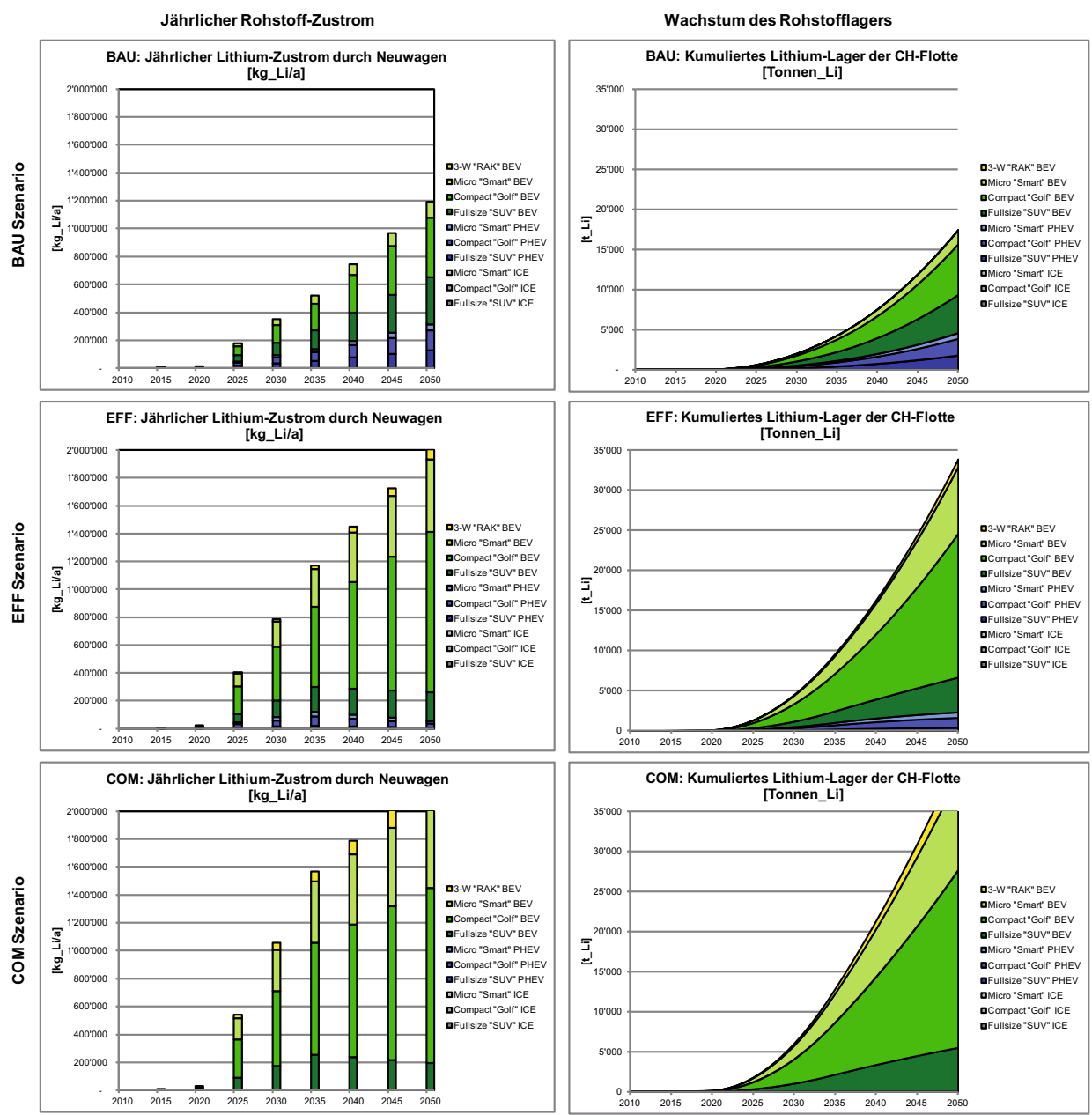

\section{Abbildung 44: Jährlicher Lithiumzustrom durch Neuwagen sowie Wachstum des entsprechenden Lithium-Rohstofflagers für die drei Szena- rien.}




\subsection{Fokus Seltenerdelemente}

\subsubsection{Anwendungen}

Seltenerdelemente (SEE) werden in vielfältigen Anwendungsbereichen eingesetzt, beispielsweise in Laser, in miniaturisierten Komponenten der ICT, in Ölraffinerien zum Umwandeln des Rohöls in Benzin, in Permanentmagneten für Windkraftanlagen, in Automobilkatalysatoren oder in der Behandlung von polierten Gläsern, Spiegeln und Präzisionslinsen (Angerer et al. 2009a; Hatch 2011). In der Mobilität werden verschiedene SEE genutzt, so etwa Neodym und Dysprosium für Permanentmagnete in Elektromotoren, Lanthan für Nickel-Metallhydrid-Akkumulatoren (NiMH) oder Cer als Katalysator zur Reduktion der COEmissionen von Verbrennungsmotoren.

\subsubsection{Globale Nachfrage}

Die Nachfrage nach SEE ist zwischen 1980 und 2009 um rund das Vierfache gestiegen. Mitte 2010 sind die Preise für SEE nach Bekanntgabe einer Verschärfung der Exportrestriktionen für SEE durch China explodiert, welches heute fast die gesamte SEE-Weltproduktion abdeckt, obwohl es nur 30 Prozent der weltweiten Vorkommen besitzt (Buller et al. 2012; Hatch 2011). Betrug beispielsweise der Preis von Neodym-Oxid Anfang 2006 noch rund 15 US-Dollar pro Kilogramm, lag er im August 2011 bei 340 US-Dollar pro Kilogramm, ging bis Mai 2012 aber wieder auf 120 US-Dollar pro Kilogramm zurück. Bei Cer wiederum hat sich der Preis von etwa 5 US-Dollar pro Kilogramm bis Mitte 2010 auf rund 150 US-Dollar pro Kilogramm im August 2011 erhöht, um dann bis Mai 2012 wieder auf 25 US-Dollar pro Kilogramm zu sinken.

Die zukünftige Nachfrage nach SEE wird wesentlich durch die technologische Entwicklung bestimmt werden. Grundsätzlich wird davon ausgegangen, dass SEE in den nächsten Jahren eine starke Nachfrage erfahren werden. Gemäss den Szenarien von U.S. DOE (2011) werden die neuen Technologien wie Windkraftanlagen und Hybrid- und Elektrofahrzeuge bis 2025 rund 40 Prozent der Neodym-Nachfrage ausmachen, gegenüber etwas mehr als 10 Prozent im Jahr 2010. Für Cer, welches u.a. in NiMH-Batterien und Autokatalysatoren eingesetzt wird, geht dieselbe Studie davon aus, dass sich die Nachfrage wegen erneuerbarer Energietechnologien und Elektromobilität zwischen 2010 und 2025 von 2,5 
auf 12 Prozent der gesamten Nachfrage erhöht. Die Nutzung von Cer bleibt also durch andere Anwendungsbereiche bestimmt.

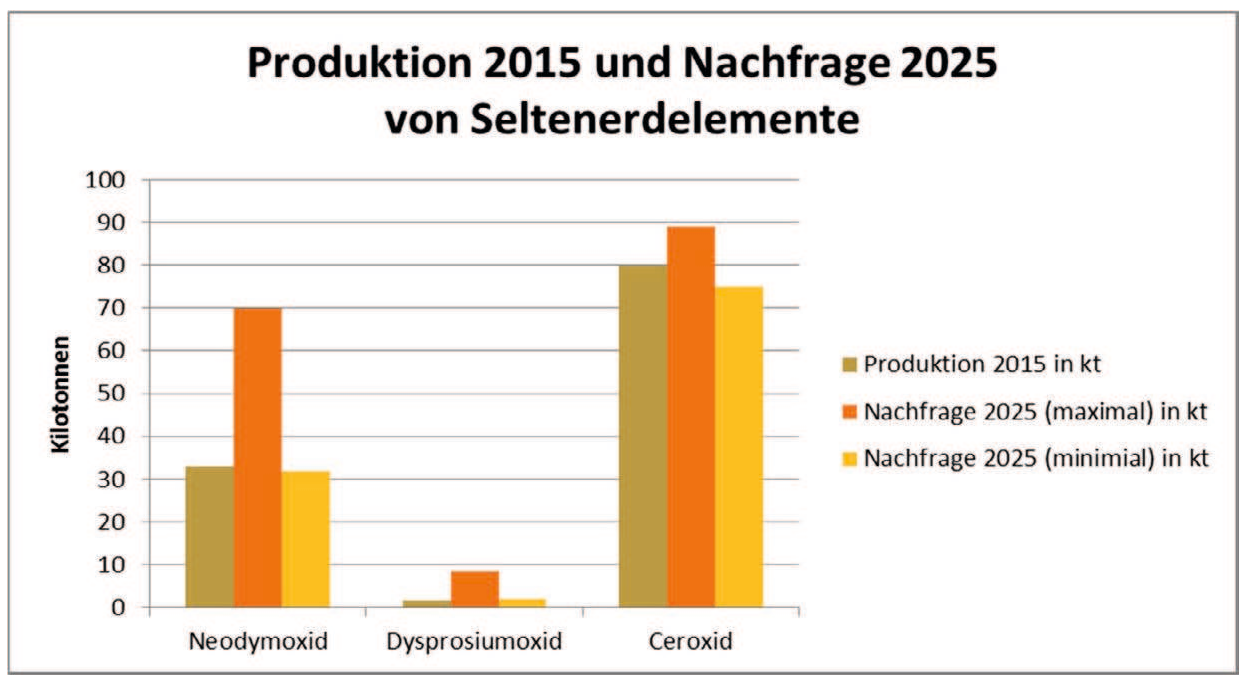

Abbildung 45: Gegenüberstellung von Produktionsniveau 2015 und minimale sowie maximale Nachfrage 2025 für ausgewählte Seltenerdelementoxide (U.S. DOE 2011).

Mit dem erwarteten starken Anstieg der Nachfrage stellt sich die Frage, ob dieser sich durch einen intensiveren Abbau von SEE decken lässt. Aktuell werden rund 95 Prozent der SEE in China abgebaut und raffiniert, doch mittlerweile ist der Aufbau von Abbau- und Raffinationskapazitäten auch in anderen Ländern wie beispielsweise den USA, Australien oder Grönland geplant oder bereits im Gange. Dadurch sollte die aktuelle Produktion von 120 Kilotonnen pro Jahr bis 2015 auf insgesamt 200 Kilotonnen im Jahr gesteigert werden können. Je nach Element sind die Potenziale jedoch sehr verschieden. Für einige Elemente, wie beispielsweise Neodym, wird das erwartete Produktionsniveau bis 2015 deutlich tiefer liegen als die erwartete maximale Nachfrage bis 2025. Bei anderen Elementen wiederum wird das Produktionsniveau bis 2015 annähernd die erwartete maximale Nachfrage bis 2025 erreichen, wie dies etwa bei Cer der Fall ist (U.S. DOE 2011; siehe Abbildung 45). 


\subsubsection{Nachfrage durch Neuwagen in der Schweiz}

Für eine Abschätzung der Nachfrage nach SEE durch die drei in dieser Studie betrachteten Szenarien am Beispiel Neodym wurde davon ausgegangen, dass Permanentmagnete (Neodym-Eisen-Bor-Magnete) im Antriebsmotor die Hauptsenke für Neodym darstellen. In einem ersten Schritt wurde deshalb zunächst der Neodymbedarf pro Masseneinheit Elektromotor ermittelt.

Unter der Annahme, dass ein Elektromotor zu 4,5 Gewichtsprozent aus Magneten und diese wiederum zu 27 Gewichtsprozent aus Neodym bestehen (Widmer und Gauch 2011), ergibt dies 0,012 kg Neodym pro Kilogramm Motor. Mithilfe der im Modell für die Szenarioberechnungen enthaltenen Motorgewichte je Fahrzeugkategorie wurde anschliessend der jeweilige Neodymbedarf pro Fahrzeugkategorie berechnet (siehe Tabelle 22) und diese wiederum mit den Fahrzeugverkäufen gemäss den drei Szenarien verknüpft. Verwendet werden dabei die Inflows in die Schweiz; die Outflows (Entsorgung bzw. Recycling) wurden nicht berücksichtigt. Aus den resultierenden jährlichen Neodymzuströmen wurden die kumulierten Neodymrohstofflager abgeleitet (siehe Abbildung 46).

In Abbildung 46 sind der jährliche Neodymzustrom durch Neuwagen sowie das Wachstum des Neodymrohstofflagers für jedes der drei Szenarien dargestellt. Wie daraus hervorgeht, steigt der jährliche Neodymzustrom durch Neuwagen von rund 0,3 Tonnen pro Jahr im Jahr 2015 auf Werte zwischen rund 82 und 88 Tonnen pro Jahr im Jahr 2050 an. Das resultierende Neodymrohstofflager im Jahr 2050 beträgt zwischen rund 1370 und 2020 Tonnen.

\begin{tabular}{|c|c|c|c|c|c|}
\hline & & 2012 & 2020 & 2035 & 2050 \\
\hline \multirow[t]{3}{*}{ Fullsize SUV } & ICE & - & - & - & - \\
\hline & PHEV & 0,90 & 0,81 & 0,73 & 0,66 \\
\hline & BEV & 0,90 & 0,81 & 0,73 & 0,66 \\
\hline \multirow[t]{3}{*}{ Compact Golf } & ICE & - & - & - & - \\
\hline & PHEV & 0,60 & 0,54 & 0,49 & 0,44 \\
\hline & BEV & 0,60 & 0,54 & 0,49 & 0,44 \\
\hline \multirow[t]{3}{*}{ Micro Smart } & ICE & - & - & - & - \\
\hline & PHEV & 0,60 & 0,54 & 0,49 & 0,44 \\
\hline & BEV & 0,60 & 0,54 & 0,49 & 0,44 \\
\hline 3-Wheeler RAKe & BEV & 0,30 & 0,27 & 0,24 & 0,22 \\
\hline
\end{tabular}

Tabelle 22: $\quad$ Neodymbedarf pro Fahrzeug (in $\mathrm{kg}$ ). 

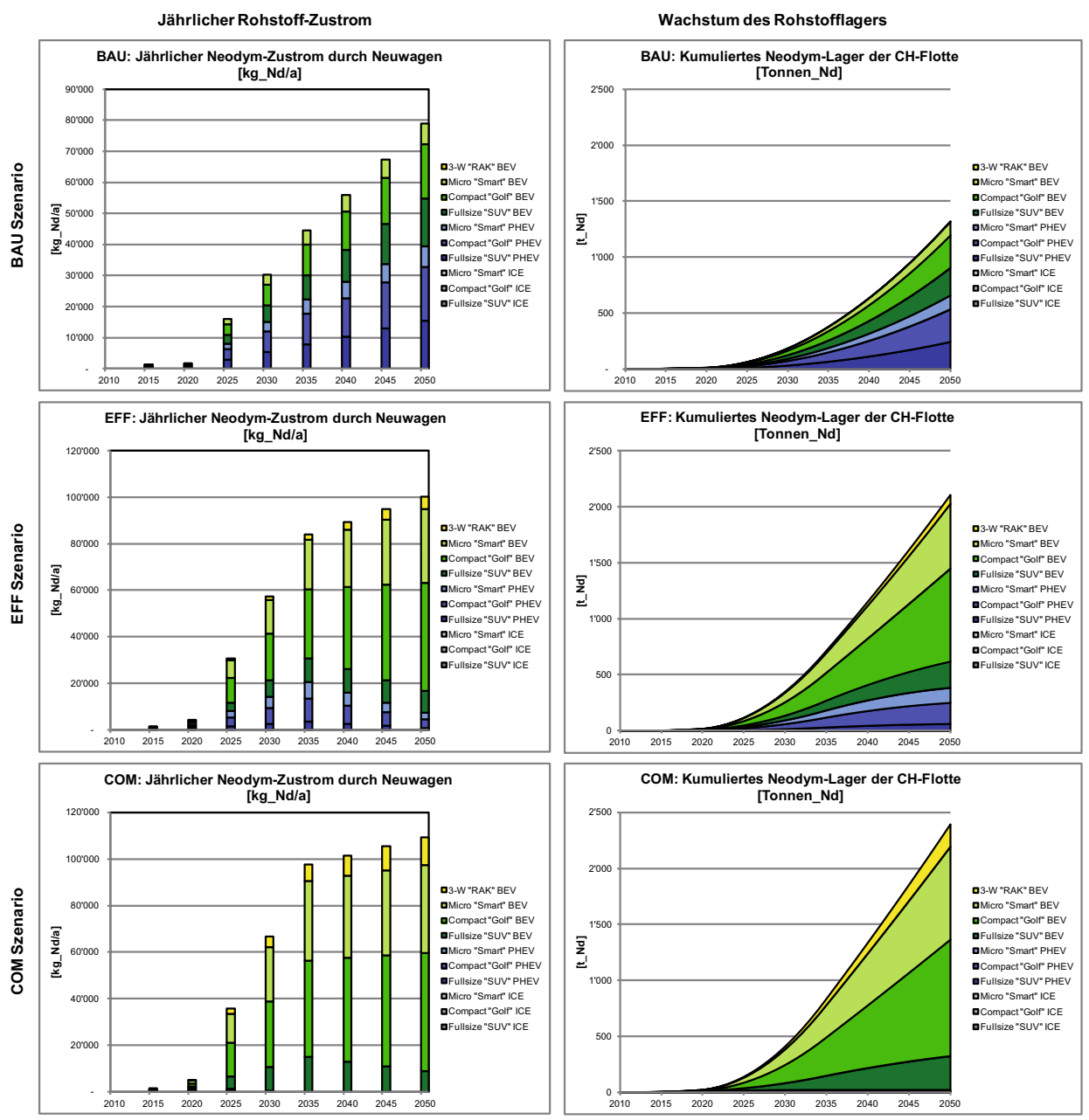

Abbildung 46: Jährlicher Neodymzustrom durch Neuwagen sowie Wachstum des entsprechenden Neodymrohstofflagers für die drei Szenarien.

\subsubsection{Mögliche Massnahmen}

Eine Entlastung der Nachfrage der SEE kann durch Substitution, effizienteren Einsatz in den jeweiligen Anwendungen und Recycling erreicht werden. 
Die Substitution von SEE wie beispielsweise Neodym oder Dysprosium ist insofern nicht trivial, als sie in starken Permanentmagneten aus heutiger Sicht höchstens durch andere SEE substituierbar sind, und eine Substitution durch andere technische Systeme wie zum Beispiel Elektromagnete zu tieferen Leistungsdichten und Wirkungsgraden führen würde (Angerer et al. 2009a; APS Panel 2009). Die Substitution kritischer SEE ist solange die beste Lösung, als das Problem damit nicht einfach auf andere kritische Rohstoffe verschoben wird. Bei einem effizienteren Einsatz wiederum gilt es grundsätzlich zu beachten, dass dies nur Sinn macht, wenn die Anstrengungen nicht mit einem erhöhten Konsum einhergehen (Reboundeffekt). Zudem kann es im Widerspruch zum Recycling stehen, wenn die Konzentrationen der Elemente in den Bauteilen so niedrig werden, dass sich eine Rückgewinnung aus energetischen Gründen nicht mehr lohnt. Eine Rückgewinnung von SEE aus Produktionsabfällen und Altfahrzeugen ist langfristig unabdingbar und insbesondere auch aus ökologischer Perspektive sinnvoll: Einerseits wird einem Verlust der Metalle entgegengewirkt, andererseits kann die Ökobilanz bei der Sekundärproduktion deutlich besser ausfallen als bei der Primärproduktion. Insbesondere entstehen bei SEE bei der Sekundärproduktion keine radioaktiven Nebenprodukte. Nicht zuletzt kann eine verstärkte Rückgewinnung dieser Metalle dabei helfen, die bestehenden Länder- und Produzentenabhängigkeiten zu reduzieren. Dies setzt jedoch unter anderem voraus, dass entsprechende Rücknahmesysteme vorhanden sind, innerhalb derer sich die Fahrzeugkomponenten abtrennen und weiterverarbeiten lassen.

Wie auch für andere Elemente, deren Lager in der Fahrzeugflotte derzeit aufgebaut werden, gilt es allerdings grundsätzlich zu beachten, dass ein Anstieg der Nachfrage nicht bzw. nur zum Teil durch eine verstärkte Rückgewinnung entlastet werden kann. Neben einer vorausschauenden Abstimmung von Nachfrage und Angebot wird es deshalb ganz wesentlich sein, dass sich die Primärproduktion in Zukunft stärker an den Prinzipien einer nachhaltigen Entwicklung orientiert und dies, etwa durch Rohstoffzertifizierungen und klare Vorgaben für Unternehmen, die im Rohstoffbereich tätig sind, unterstützt wird. Dies nicht zuletzt, weil verschiedene Projekte zur Erschliessung neuer Lagerstätten in Vorbereitung sind und mittel- bis langfristig mit einer Verschlechterung der Qualität der abgebauten Lagerstätten zu rechnen ist, was mit einem höheren materiellen und energetischen Aufwand bei der Rohstoffgewinnung einhergeht. 


\subsection{Fokus Platinmetalle}

\subsubsection{Anwendungen}

Zurzeit die bedeutsamste Mobilitätsanwendung von Platinmetallen sind Abgaskatalysatoren für Verbrennungsmotoren. Weitere Anwendungsbereiche sind Elektronik und Elektrotechnik, Schmuck, Chemie und Petrochemie, Brennstoffzellen, Dentaltechnik oder optische Gläser. In der Elektromobilität wird Platin als Katalysator in Brennstoffzellen genutzt (Buller et al., 2012; Wäger et al. 2010). Die Produktion von Platinmetallen hat in den Jahren vor 2009 um durchschnittlich 3,6 Prozent pro Jahr zugenommen. Die Preise sind zwischen 2003 und 2007 zum Teil stark gestiegen. Während sich der Preis für Platin verdoppelte, hat sich der Preis für Ruthenium fast verzwanzigfacht. Gemäss IZT (2009) werden die Preise stark von den Produzenten bestimmt und haben nichts mit der geologischen Knappheit oder den Kosten der Förderung und Verarbeitung der Elemente zu tun.

\subsubsection{Globale Nachfrage}

Für die Zukunft wird erwartet, dass sich der Bedarf an Platinmetallen durch die Nachfrage nach Zukunftstechnologien wie beispielsweise Brennstoffzellen in Elektrofahrzeugen oder miniaturisierte Kondensatoren weiter erhöht (Angerer et al. 2009a). Gemäss World Energy Outlook (IEA 2011) ist in Nicht-OECD-Ländern eine starke Vergrösserung der Fahrzeugflotte zu erwarten, die zum grössten Teil durch Fahrzeuge mit konventionellem Antrieb gedeckt werden dürfte. Der Anteil von Elektro- und Hybridautos würde dabei im Jahr 2035 in NichtOECD-Ländern 20 Prozent entsprechen und in OECD-Ländern 35 Prozent (IEA 2011). Damit sollte das Anwendungsgebiet der Autoabgaskatalysatoren kurz- bis mittelfristig bei der Nachfrage nach Platinmetallen weiterhin eine zentrale Rolle spielen.

Soweit die Produktionskapazitäten mit der erwarteten Nachfrage mithalten, ist bei Platinmetallen vorderhand nicht mit einer Knappheit zu rechnen (Angerer et al. 2009a). Allerdings sind auch hier gewisse Länder- und Produzentenabhängigkeiten vorhanden, die Risiken für die Versorgung beinhalten: Platinmetalle werden hauptsächlich in Südafrika (2009: 79 Prozent der Weltjahresproduktion) und Russland gewonnen. 


\subsubsection{Nachfrage durch Neuwagen in der Schweiz}

Für eine Abschätzung der Nachfrage nach Platinmetallen durch die drei in dieser Studie betrachteten Szenarien wurde davon ausgegangen, dass Autokatalysatoren die Hauptsenke für diese drei Elemente darstellen. Damit beschränkt sich die Betrachtung auf die drei Platinmetalle Palladium, Platin und Rhodium. Es wird kein Unterschied zwischen Katalysatoren für Diesel- (die vor allem Pt enthalten) und Benzinfahrzeugen (vor allem Pd und Rh) gemacht - d.h., es wird unabhängig vom Fahrzeugtyp der globale Mix an Palladium, Platin und Rhodium in Katalysatoren verwendet. In einem ersten Schritt wurde deshalb der Massengehalt dieser drei Elemente in einer Masseneinheit Verbrennungsmotor ermittelt. Unter der Annahme, dass etwa 5 Gramm dieser drei Platinmetalle in einem durchschnittlichen Fahrzeug mit einem 75-Kilogramm-Motor enthalten sind, ergibt dies 0,07 Gramm Platinmetalle pro Kilogramm Motor. Mithilfe der im Modell für die Szenarienberechnungen enthaltenen Motorgewichte pro Fahrzeugkategorie wurde der jeweilige Platinmetallbedarf pro Fahrzeugkategorie berechnet (siehe Tabelle 23) und diese wiederum mit den Fahrzeugverkäufen gemäss der drei Szenarien verknüptt (auch hier gehen die Inflows in die Schweiz ein, die Outflows [Entsorgung bzw. Recycling] bleiben unberücksichtigt). Aus den resultierenden jährlichen Platinmetallzuströmen wurden die kumulierten Platinmetallrohstofflager abgeleitet.

\begin{tabular}{|l|c|c|c|c|c|}
\hline \multirow{4}{*}{ Fullsize SUV } & & $\mathbf{2 0 1 2}$ & $\mathbf{2 0 2 0}$ & $\mathbf{2 0 3 5}$ & $\mathbf{2 0 5 0}$ \\
\cline { 2 - 6 } & ICE & 0,0088 & 0,0079 & 0,0071 & 0,0064 \\
\cline { 2 - 6 } & PHEV & 0,0053 & 0,0047 & 0,0043 & 0,0038 \\
\cline { 2 - 6 } & BEV & - & - & - & - \\
\hline \multirow{4}{*}{ Compact Golf } & ICE & 0,0053 & 0,0047 & 0,0043 & 0,0038 \\
\cline { 2 - 6 } & PHEV & 0,0035 & 0,0032 & 0,0028 & 0,0026 \\
\cline { 2 - 6 } & BEV & - & - & - & - \\
\hline \multirow{4}{*}{ Micro Smart } & ICE & 0,0053 & 0,0047 & 0,0043 & 0,0038 \\
\cline { 2 - 6 } & PHEV & 0,0035 & 0,0032 & 0,0028 & 0,0026 \\
\cline { 2 - 6 } & BEV & - & - & - & - \\
\hline 3-Wheeler RAKe & BEV & - & - & - & - \\
\hline
\end{tabular}

Tabelle 23: $\quad$ Platinmetallbedarf pro Fahrzeug (in $\mathrm{kg}$ ).

In Abbildung 47 sind der jährliche Platinmetallzustrom durch Neuwagen sowie das Wachstum des Platinmetallrohstofflagers für jedes der drei Szenarien dargestellt. Wie daraus hervorgeht, sinkt der jährliche Platinmetallzustrom durch Neu- 
wagen von rund 1,8 t/a im Jahr 2015 auf Werte zwischen rund 0,5 t/a und 0,8 t/a im Jahr 2050. Das resultierende Platinmetallrohstofflager im Jahr 2050 beträgt zwischen rund 48 und 57 Tonnen.
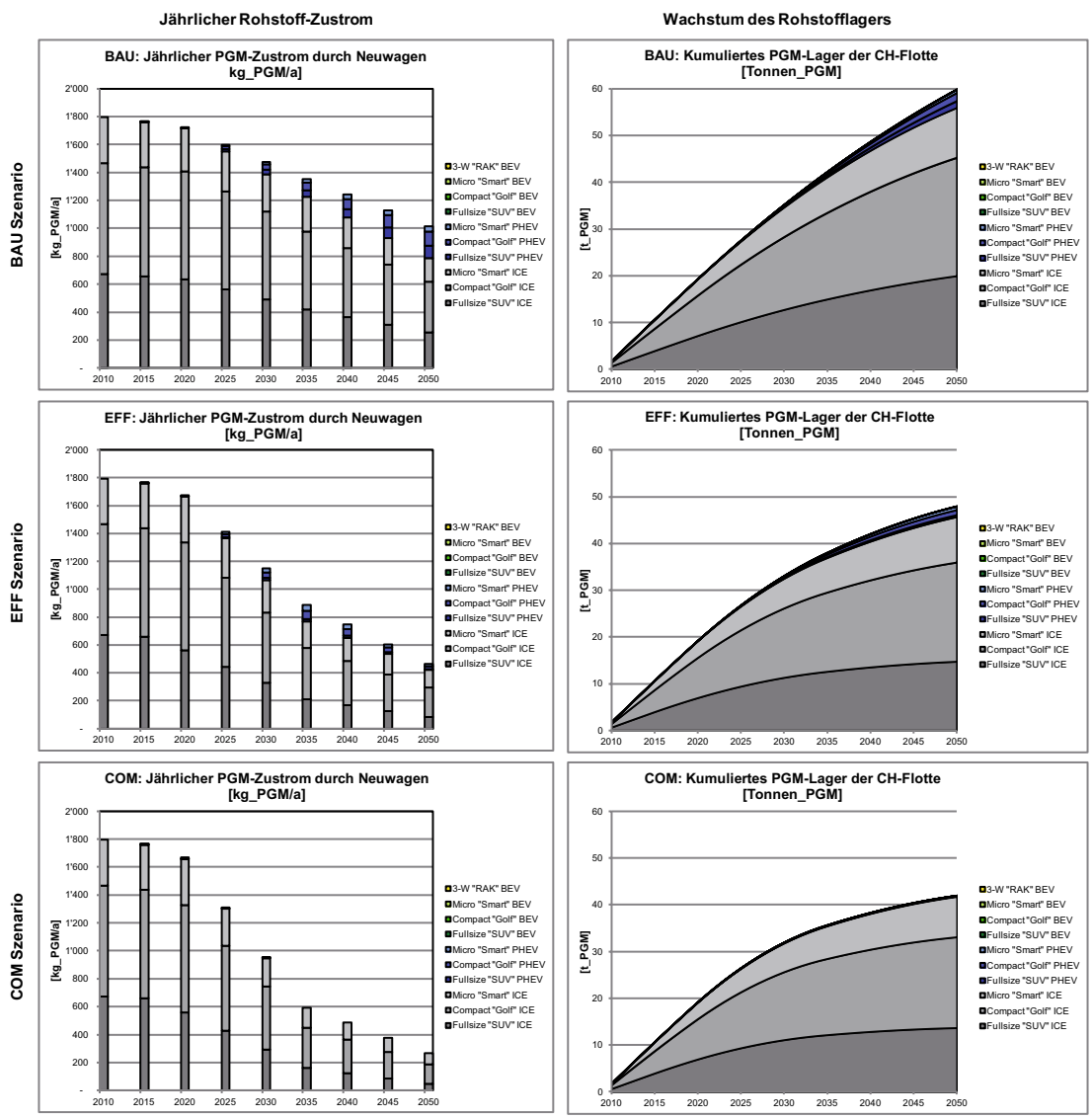

Abbildung 47: Jährlicher Platinmetallzustrom durch Neuwagen sowie Wachstum des entsprechenden Platinmetallrohstofflagers für die drei Szenarien.

\subsubsection{Mögliche Massnahmen}

Aufgrund ihres hohen Marktpreises ist die Rückgewinnung von Platinmetallen vor allem im Bereich der Produktionsabfälle bereits heute etabliert. Bei den EoL- 
Produkten sind die Recyclingraten zwar deutlich höher als beispielsweise bei Lithium oder Seltenerdelemente, unter anderem wegen des häufigen Wechsels der Produkteigentümer und des globalen Altgerätehandels besteht jedoch noch Handlungsbedarf (Wäger et al. 2010; UNEP 2011). Wie am Beispiel von Palladium gezeigt wurde, schneidet die Rückgewinnung seltener Edelmetalle aus EoLProdukten wie Elektro- und Elektronikaltgeräten aus ökologischer Sicht deutlich besser ab als ihre Gewinnung aus Erzen (Wäger et al. 2011b). Hinsichtlich einer möglichen Substitution von Platinmetallen könnten in Zukunft alkalihaltige Brennstoffe an Bedeutung gewinnen, da sie auch mit nicht platinhaltigen Katalysatoren wie Silber und Gold funktionieren (Angerer et al. 2009a).

\subsection{Fazit}

Die Hauptursache für die Kritikalität bestimmter mineralischer Rohstoffe ist weniger deren geologische Verfügbarkeit als vielmehr die Konzentration des Angebots auf wenige Länder und Firmen, verbunden mit fehlenden Substitutionsmöglichkeiten und geringen Recyclingraten.

Es ist davon auszugehen, dass die Elektromobilität zu einer Zunahme der Nachfrage nach kritischen oder potenziell kritischen Rohstoffen führen wird. Besonders deutlich wird die Zunahme bei Lithium ausfallen, dessen globale Nachfrage wesentlich durch die Elektromobilität sowie andere Elektro- und Elektronikgeräte bestimmt sein wird. Aber auch die Seltenerdelemente könnten im Bereich der Elektromobilität vermehrt zur Anwendung kommen, beispielsweise in leistungsfähigen Permanentmagneten.

Inwieweit die erwartete Nachfragesteigerung durch die Elektromobilität tatsächlich zu Versorgungsproblemen bei kritischen Rohstoffen führen könnte, hängt von verschiedenen Faktoren ab. Dazu zählen etwa die geografische Konzentration der Produktion, technologische Entwicklungen bei der (Rück-)Gewinnung der Rohstoffe, die Geschwindigkeit der Anpassung von Primärproduktionskapazitäten, der Aufbau einer geeigneten Recyclinginfrastruktur oder die Substitution durch andere Elemente oder Technologien.

Einer Zunahme der Nachfrage nach kritischen Rohstoffen kann durch verschiedene Strategien begegnet werden, darunter eine Erhöhung der Primärproduktionskapazitäten, ein verstärktes Recycling, ein effizienterer Einsatz der Rohstoffe oder Substitution. 
Sofern dem erwarteten Anstieg des Bedarfs nach seltenen Metallen nicht z.B. durch Substitution entgegengewirkt werden kann, wird die Deckung der Nachfrage weiterhin vor allem durch die Primärproduktion erfolgen müssen. Mit fortschreitendem Abbau der heute genutzten Primärressourcen wird aber der materielle und energetische Aufwand für die Primärproduktion steigen. Neben einer vorausschauenden Abstimmung von Nachfrage und Angebot wird es deshalb ganz wesentlich sein, dass sich die Primärproduktion metallischer Rohstoffe in Zukunft viel stärker an Prinzipien einer nachhaltigen Entwicklung orientiert und ihre ökologischen und sozialen Auswirkungen auf ein Minimum reduziert werden. Die Schweiz produziert diese Primärrohstoffe nicht, sondern importiert sie; als Sitz einiger der weltweit grössten Rohstoffkonzerne spielt sie überdies eine Rolle in Finanzierung und Handel. 


\section{$7 \quad$ Nachhaltigkeitsbeurteilung}

\subsection{Wirtschaftliche Relevanz der Automobil- branche}

Die motorisierte individuelle Mobilität stellt einen der grössten Wirtschaftssektoren der Schweiz dar. Allein für Personenwagen wurden 2011 rund 11 Milliarden Franken für Benzin und Diesel ausgegeben, weitere rund 12,9 Milliarden Franken kostete der Kauf neuer Autos. Die kantonalen Verkehrsabgaben betrugen über 2 Milliarden Franken, Versicherungen über 4 Milliarden Franken und der Umsatz des Garagengewerbes für Personenwagen machte rund 14 Milliarden Franken aus. Mit dem Transportgewerbe zusammen setzten über $225000 \mathrm{Be}-$ schäftigte in über 20000 Betrieben rund 90 Milliarden Franken um (Strasseschweiz 2012), das sind rund 16 Prozent des Bruttoinlandprodukts der Schweiz. Die Entwicklung von neuen Technologien wie der Elektromobilität birgt grosse Chancen für die Schweizer Wirtschaft und Gesellschaft. In verschiedenen Bereichen kann die Schweizer Wirtschaft entscheidendes Know-how einbringen und neue Entwicklungen prägen: Von den oben genannten 90 Milliarden Franken entfallen 14 Milliarden auf Schweizer Zulieferbetriebe für die internationale Automobilindustrie.

Gerade im Bereich der Elektromobilität ist die Schweiz aufgrund ihrer optimalen geografischen und politischen Rahmenbedingungen ein idealer Standort für Forschung und Innovation. Durch innovative Forschungsprojekte können Einsatzbereiche und Geschäftsmodelle für Elektromobilität sowie die nötigen sozioökonomischen Innovationsschritte erprobt werden. Auch für die Herstellung von spezifischen Produkten für die Elektromobilität wie beispielsweise Leistungselektronik, Komponenten oder ganze Fahrzeuge eignet sich die Schweiz als spezialisierter Bildungsstandort, was zu neuen Arbeitsplätzen und zusätzlicher Wertschöpfung für die Volkswirtschaft führen kann.

Während der Nutzungsphase der Elektrofahrzeuge profitieren die Anwohnenden von tieferen Lärm- und Schadstoffemissionen. Ausserdem kann die für die Elektromobilität benötigte Elektrizität innerhalb der Schweiz produziert werden und führt damit zu neuen Arbeitsplätzen und einer geringeren Abhängigkeit von importierten fossilen Rohstoffen. 
Schliesslich ergeben sich auch durch das Recycling verschiedene Möglichkeiten für die Schweizer Wirtschaft. Durch Innovation und die Weiterführung der schweizerischen Recycling- und Entsorgungsbranche können weitere Arbeitsplätze geschaffen und der Wirtschaftsstandort Schweiz gestärkt werden.

Für die verschiedenen Akteure der Schweizer Volkswirtschaft sind diese Entwicklungen meist positiv. Während die Wirtschaft von einem stärkeren Standort, neuen Aktivitäten und Arbeitsplätzen profitiert, kann die Gesellschaft von neuen Arbeitsmöglichkeiten und einer höheren Lebensqualität ausgehen. Auch der Bund profitiert durch höhere allgemeine Steuereinnahmen, was den Wirtschaftsstandort Schweiz stärkt und die Wertschöpfung erhöht. Ausserdem können dank der Elektromobilität Abhängigkeiten zu Ländern, die fossile Energien liefern, teilweise reduziert werden. Eine grosse Schwierigkeit für den Bund sind die reduzierten Einnahmen aus der Mineralölsteuer, die durch neue Steuermodelle zu kompensieren sein werden (siehe Exkurs).

\section{Exkurs: Mineralölsteuer}

Die Mineralölsteuer wird auf Treib- und Brennstoffe erhoben und ist eine wichtige Einnahmequelle für den Bund, die zu 70 Prozent in die Finanzierung der Verkehrsinfrastruktur (neben Strasse auch ÖV) fliesst, zu 30 Prozent in die allgemeine Bundeskasse. Der Gesamtertrag von jährlich über fünf Milliarden Franken entspricht rund 8 Prozent der totalen Bundeseinnahmen.

Die Einnahmen aus der Mineralölsteuer werden sowohl durch die Effizienzgewinne der Fahrzeuge wie auch durch den verstärkten Markteintritt der Elektrofahrzeuge zurückgehen. Wie stark dieser Rückgang ausfällt, hängt von der Effizienzsteigerung sowie der Entwicklung des Marktanteils der Elektromobilität ab. Je effizienter die treibstoffbetriebenen Fahrzeuge werden und je schneller sich die Elektromobilität in der Schweiz durchsetzt, desto höher ist der Rückgang der Mineralölsteuereinnahmen. Nachfolgende Abbildung zeigt für die verschiedenen Szenarien die Entwicklung der Einnahmen aus der Mineralölsteuer.

Gemäss den Berechnungen macht die Mineralölsteuer von PKW im Jahr 2015 rund 3,4 Milliarden Franken aus. In allen drei Szenarien sinken die Mineralölsteuereinnahmen signifikant. Beim BAU-Szenario würden die Einnahmen im Jahr 2035 auf rund 2,1 Milliarden Franken sinken 62 Prozent der Einnahmen im Jahr 2015. Beim Szenario EFF gehen die Erträge auf 58 Prozent (1,9 Milliarden Franken im Jahr 2035) zurück. Die Einnahmen im COM-Szenario liegen im Jahr 2035 bei nur noch 1,5 Milliarden Franken oder 45 Prozent der Einnahmen im Jahr 2015. 


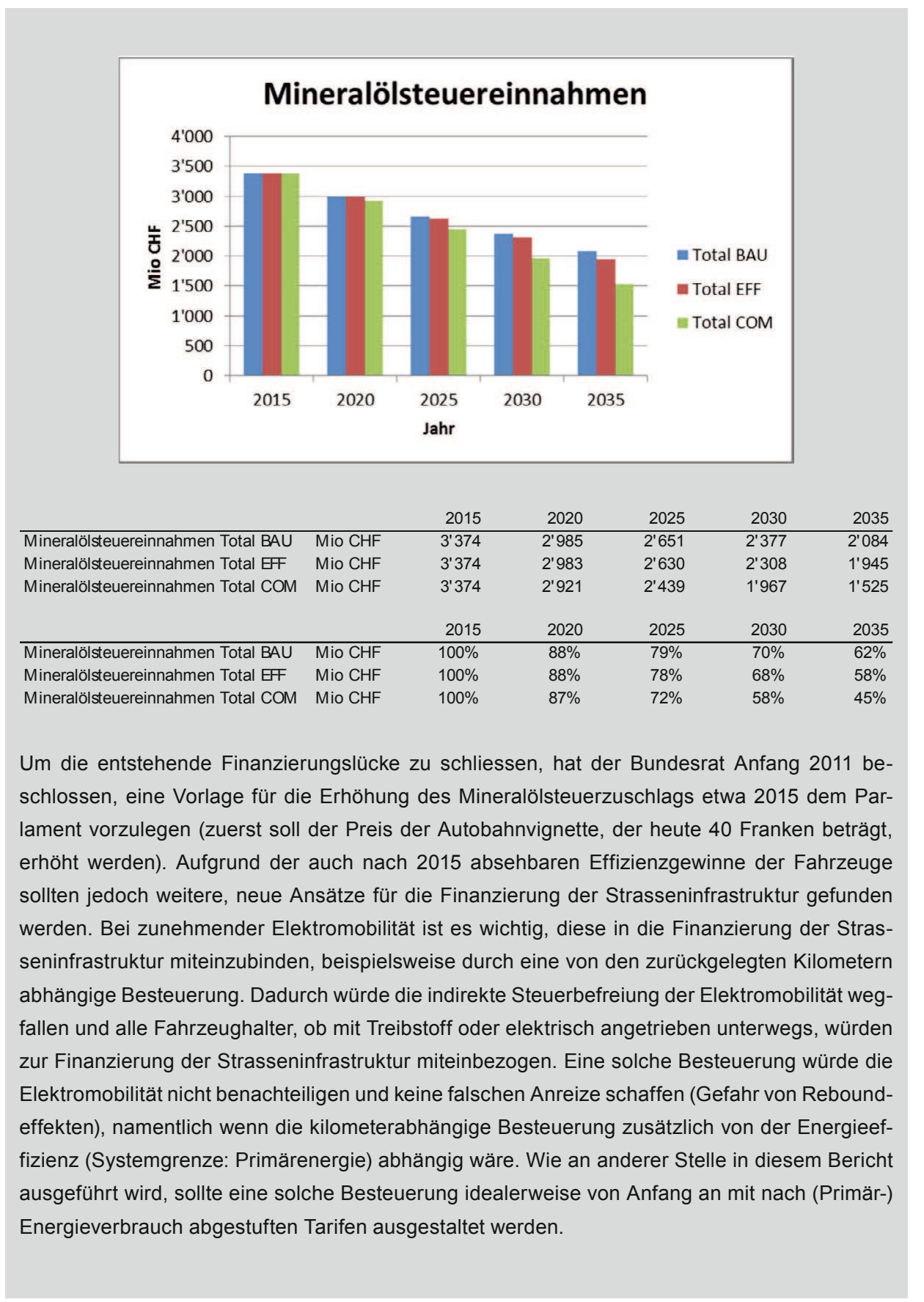




\subsection{Methodik}

Die Einflüsse der Elektromobilität auf Umwelt, Wirtschaft und Gesellschaft werden im Folgenden anhand des bestehenden Beurteilungssystems der Nachhaltigkeitsindikatoren für Strasseninfrastrukturprojekte (NISTRA; siehe ASTRA 2003) evaluiert. Im Vergleich zur ReCiPe-Methodik beinhaltet diese Methode nicht nur Indikatoren zu den Umwelteinflüssen und Ressourcen, sondern auch zu Einflüssen auf Wirtschaft und Gesellschaft. Damit wird eine Gesamtanalyse möglich, die die verschiedenen Nachhaltigkeitsaspekte miteinbezieht.

\begin{tabular}{|c|c|c|c|}
\hline \multirow{2}{*}{\multicolumn{2}{|c|}{$\begin{array}{l}\text { Oberziele } \\
\text { Umwelt }\end{array}$}} & \multicolumn{2}{|c|}{ Teilziele } \\
\hline & & & \\
\hline \multirow[t]{2}{*}{ U1 } & \multirow[t]{2}{*}{ Umweltbelastungen senken } & U1.1 & Luftschadstoffe senken \\
\hline & & U1.2 & Lärmbelastung senken \\
\hline U2 & Klima schützen & U2.1 & Treibhausgasemissionen senken \\
\hline \multirow[t]{3}{*}{ U3 } & \multirow[t]{3}{*}{ Ressourcen schonen } & U3.1 & Primärenergieverbrauch senken \\
\hline & & U3.2 & Bodenversiegelung reduzieren \\
\hline & & U3.3 & Einsatz metallischer Rohstoffe minimieren \\
\hline \multirow[t]{2}{*}{ U4 } & \multirow[t]{2}{*}{ Natürliche Vielfalt fördern } & U4.1 & Natürliche Lebensräume erhalten \\
\hline & & U4.2 & Landschaftszerschneidung reduzieren \\
\hline \multicolumn{4}{|c|}{ Wirtschaft } \\
\hline \multirow[t]{2}{*}{ W1 } & \multirow[t]{2}{*}{ Kosten minimieren } & W1.1 & Investitions- und Betriebskosten für Fahrzeuge minimieren \\
\hline & & W1.2 & Investitions- und Betriebskosten für Infrastruktur minimieren \\
\hline \multirow[t]{2}{*}{ W2 } & \multirow[t]{2}{*}{ Wertschöpfung erhöhen } & W2.1 & Absatzmärkte für Schweizer Unternehmen schaffen \\
\hline & & W2.2 & Einheimische Rohstoffe und Produkte nutzen \\
\hline \multirow[t]{2}{*}{ W3 } & \multirow[t]{2}{*}{$\begin{array}{l}\text { Indirekte wirtschaftliche } \\
\text { Effekte optimieren }\end{array}$} & W3.1 & $\begin{array}{l}\text { Unterstützung einer regional ausgeglichenen wirtschaftlichen } \\
\text { Entwicklung }\end{array}$ \\
\hline & & W3.2 & $\begin{array}{l}\text { Flexibilität des Systems erhöhen (Investitionsrisiko reduzieren, } \\
\text { Anpassungsfähigkeit bei technolog. Entwicklungen) }\end{array}$ \\
\hline \multirow[t]{2}{*}{ W4 } & \multirow{2}{*}{$\begin{array}{l}\text { Versorgungssicherheit } \\
\text { gewährleisten }\end{array}$} & W4.1 & Abhängigkeit von einzelnen Energiequellen senken \\
\hline & & W4.2 & Verfügbarkeit der Rohstoffe gewährleisten \\
\hline \multicolumn{4}{|c|}{ Gesellschaft } \\
\hline \multirow[t]{3}{*}{ G1 } & \multirow{3}{*}{$\begin{array}{l}\text { Gesundheit und } \\
\text { Wohlbefinden fördern }\end{array}$} & G1.1 & Verkehrssicherheit erhöhen \\
\hline & & G1.2 & Minimierung von Störfallrisiken \\
\hline & & G1.3 & $\begin{array}{l}\text { Beeinträchtigungen des Siedlungs- und Landschaftsbildes } \\
\text { minimieren }\end{array}$ \\
\hline G2 & $\begin{array}{l}\text { Gesellschaftliche } \\
\text { Solidarität fördern }\end{array}$ & G2.1 & $\begin{array}{l}\text { Zugang für alle Regionen und gesellschaftlichen Gruppen } \\
\text { sicherstellen }\end{array}$ \\
\hline
\end{tabular}

Tabelle 24: $\quad$ Zielsystem für die Auswirkungsanalyse. 
NISTRA basiert auf dem «Ziel- und Indikatorensystem Nachhaltiger Verkehr» des UVEK (ZINV; siehe UVEK 2008) und dient zur Evaluation von Strasseninfrastrukturprojekten und deren Auswirkungen auf die Dimensionen Wirtschaft, Gesellschaft und Umwelt. Für jede dieser Dimensionen sind spezifische Oberziele und Teilziele definiert.

Aufgrund der spezifischen Charakteristiken der Elektromobilität wurde eine Auswahl der Teilziele getroffen. Tabelle 24 zeigt das in dieser Studie verwendete Zielsystem mit den entsprechenden Ober- und Teilzielen.

Um die Entwicklung der Elektromobilität evaluieren zu können, wird die Elektromobilität (EV) mit einer zukünftigen treibstoffbasierten Mobilität (ICE) verglichen. Dabei wird jeweils der gesamte Lebenszyklus der Mobilität miteinbezogen. Dies beinhaltet beispielsweise den Rohstoffabbau und die Herstellung der Fahrzeuge, die Nutzung und die Entsorgung der Fahrzeuge sowie die Bereitstellung der nötigen Energie und Elektrizität für den Betrieb der Fahrzeuge. Die Analyse zeigt auf, wie sich die Elektromobilität und die zukünftig treibstoffbasierte Mobilität innerhalb des Zielsystems entwickeln und welche Auswirkungen in den drei Dimensionen zu erwarten sind. Die Evaluation konzentriert sich auf die Mobilitätsentwicklung in der Schweiz.

Im Folgenden werden die verschiedenen Ober- und Teilziele kurz beschrieben und die Auswirkungen auf eine eher treibstoffbasierte Mobilität versus die Elektromobilität evaluiert. Eine zusammenfassende qualitative Beurteilung jedes Teilziels erfolgt aufgrund einer Beurteilungsskala, die aufzeigt, ob die Entwicklung der entsprechenden Mobilitätsentwicklung der Zielrichtung entspricht.

\begin{tabular}{|c|l|}
\hline$\checkmark$ & Starke Entwicklung gemäss Zielrichtung \\
\hline$(\checkmark)$ & Tendenzen gemäss Zielrichtung \\
\hline$\approx$ & Keine relevanten Auswirkungen \\
\hline$+/-$ & Auswirkungen von konkreter Ausgestaltung abhängig \\
\hline $\boldsymbol{\square}$ & Entwicklung gegen Zielrichtung \\
\hline
\end{tabular}

Tabelle 25: Beurteilungsskala. 


\subsection{Umwelt}

\section{U1 Umweltbelastung senken}

Lokale, nationale sowie grenzüberschreitende Umweltbelastungen sollen langfristig auf ein umweltverträgliches Niveau gesenkt werden. Die grössten Umweltauswirkungen des Verkehrs betreffen die Luftschadstoffe, die Treibhausgasemissionen und die Lärmbelastung. Die Auswirkungen auf das Klima werden unter dem separaten Kriterium U2 Klima schützen bewertet sowie die weiteren Auswirkungen im Bereich natürliche Ressourcen und natürliche Vielfalt.

\section{U1.1 Luftschadstoffe}

Luftschadstoffe werden sowohl im Betrieb von Fahrzeugen ausgestossen als auch bei der Bereitstellung der Energie, der Infrastruktur und der Fahrzeuge. Durch die seit den 1990er-Jahren bestehende Luftreinhalteverordnung (LRV) und die Abgasnormen (Euronormen) konnten die Luftschadstoffe Stickoxide, PM10, Kohlenmonoxid, Kohlenwasserstoffe und Schwefeldioxid in den letzten Jahren stark reduziert werden. Dies gilt auch für den Sekundärschadstoff Ozon, der von Autos nicht direkt ausgestossen wird, sich aber aus Vorläufersubstanzen (Stickoxide und Kohlenwasserstoffe) bildet. Das Treibhausgas $\mathrm{CO}_{2}$ ist für Mensch und Tier nicht toxisch und wird deshalb nicht zu den Luftschadstoffen im engeren Sinn gezählt. Die Beurteilung der Treibhausgasemissionen erfolgt unter dem Teilziel U2.1 Treibhausgasemissionen senken.

In Zukunft werden die Luftschadstoffe bei der treibstoffbasierten Mobilität weiter zurückgehen. Dies basiert hauptsächlich auf weiteren politischen Massnahmen sowie technischen Neuerungen und Effizienzsteigerungen der Fahrzeuge.

Da die Elektromobilität keine Luftschadstoffe emittiert, werden die Emissionen von Schadstoffen beim Betrieb der Fahrzeuge stark reduziert. Je nach Strommix kann jedoch der für die Elektromobilität benötigte Strom indirekt eine höhere Umweltbelastung verursachen und Luftschadstoffe emittieren. Mit den Zukunftsszenarien der Stromversorgung in der Schweiz ist jedoch nicht damit zu rechnen, dass eine Steigerung der Elektromobilität zusätzliche umweltbelastende Stromerzeugung bewirkt, da der zusätzliche Strombedarf bereits in den Energieszenarien mitberücksichtigt ist. 


\begin{tabular}{|c|c|c|c|}
\hline & & ICE & EV \\
\hline U1.1 & Luftschadstoffe senken & $(\checkmark)$ & $\checkmark$ \\
\hline
\end{tabular}

\section{U1.2 Lärmbelastung}

Lärm entsteht in erster Linie beim Betrieb der Fahrzeuge, einerseits durch den Motor, andererseits aber auch durch Reifen-, Fahrbahn- und Windgeräusche. Zusätzlich beeinflussen auch Reifen, Belag, Aerodynamik der Fahrzeugkarosserie und Fahrverhalten den Lärmpegel.

Bei einer vorwiegend treibstoffbasierten Mobilität wird sich der Lärm aufgrund optimierter Techniken und Innovationen in Zukunft reduzieren.

Bei der Elektromobilität nimmt die Lärmbelastung deutlich ab, da der Motorenlärm des Gesamtverkehrs signifikant leiser wird. Bei den anderen Quellen der Lärmbelastung hängt diese stark vom Fahrzeugmodel und den Reifen ab. Sie unterscheidet sich bei einem Elektrofahrzeug nicht zwingend von einem treibstoffbasierten Fahrzeug.

\begin{tabular}{|c|c|c|c|}
\hline & & ICE & EV \\
\hline U1.2 & Lärmbelastung senken & $(\vee)$ & $\checkmark$ \\
\hline
\end{tabular}

\section{U2 Klima schützen}

Treibhausgasemissionen und insbesondere $\mathrm{CO}_{2}$-Emissionen führen zu einer Veränderung des Klimas. Um schwerwiegende Folgen der Klimaänderung zu verhindern, muss die Belastung durch Treibhausgase reduziert werden. Die Schweiz hat sich verpflichtet, bis 2020 die Treibhausgasemissionen um bis zu 20 Prozent gegenüber den Emissionen des Jahres 1990 zu reduzieren.

\section{U2.1 Treibhausgasemissionen senken}

Treibhausgasemissionen entstehen bei der Nutzung des Fahrzeugs, bei seiner Herstellung, bei der Energiebereitstellung und auch beim Bau der Infrastruktur. Treibstoffbasierte Fahrzeuge werden in Zukunft effizienter und dadurch wird sich der Treibhausgasausstoss reduzieren. Das neue $\mathrm{CO}_{2}$-Gesetz hat den Zielwert der Treibhausgasemissionen von neu immatrikulierten Personenwagen bis 2015 
auf 130 Gramm pro Kilometer festgelegt. Ziel ist es, bis 2020 die Treibhausgasemissionen des Verkehrs gegenüber 1990 um 20 Prozent zu senken. Durch effizientere Fahrzeuge kann der Treibstoffverbrauch verringert werden. Dies wirkt sich auch auf die Energiebereitstellung aus, die durch die tiefere Treibstoffnachfrage ebenfalls reduziert wird und weniger Treibhausgase emittiert.

Elektromotoren sind von ihrem Wirkungsgrad her deutlich effizienter als Verbrennungsmotoren, daher haben sie das Potenzial, die Treibhausgasemissionen zu senken. Die Höhe der Emissionen hängt jedoch davon ab, wie die im Fahrzeug eingesetzte Elektrizität hergestellt wird. Wie bereits erwähnt, wird die zukünftige Stromversorgung der Schweiz weiterhin grösstenteils klimaneutral sein und eine Steigerung der Elektromobilität bewirkt keine zusätzliche umweltbelastende Stromerzeugung.

Bei der Herstellung der Fahrzeuge und der nötigen Infrastruktur werden keine grossen Unterschiede zwischen treibstoffbasierten Fahrzeugen und Elektromobilen erwartet.

\begin{tabular}{l|c|c|c}
\hline & & ICE & EV \\
\hline U2.1 & Treibhausgasemissionen senken & $(\checkmark)$ & $\checkmark$ \\
\hline
\end{tabular}

\section{U3 Ressourcen schonen}

Die Effizienz der Rohstoff- und Energienutzung im gesamten Lebenszyklus eines Produktes soll verbessert werden (Material- und Energieintensität). Dazu muss der Energieverbrauch verringert beziehungsweise durch erneuerbare Energieträger substituiert werden. Gleichzeitig sollen der Abbau natürlicher Ressourcen und die Bodenversiegelung vermindert werden.

\section{U3.1 Primärenergieverbrauch senken}

Treibstoffbasierte Mobilität beruht auf fossiler Energie. Durch die steigende Energieeffizienz und den sinkenden Treibstoffverbrauch wird der Primärenergieverbrauch sinken. Die Elektromobilität hat einen deutlich geringeren Primärenergieverbrauch, da diese mit Strom betrieben wird. Auch hier hängt der Primärenergieverbrauch vom Strommix der Elektrizität ab. Da dieser in der Schweiz aufgrund der Zukunftsszenarien zu einem hohen Anteil aus erneuerbaren und 
klimaneutralen Energien besteht, wird der Primärenergieverbrauch bei der Elektromobilität stark sinken.

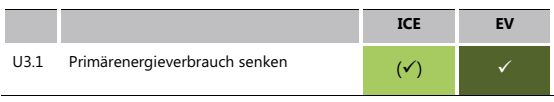

\section{U3.2 Bodenversiegelung reduzieren}

Durch den Bau von Strassen, asphaltierten Wegen und Plätzen, Häusern, Gewerbe- und Industrieanlagen werden freie Flächen in grossem Umfang versiegelt. Eine starke Bodenversiegelung wirkt sich negativ auf den Wasserhaushalt aus und kann zu einer höheren Grundwasserbelastung führen, da bei punktueller Versickerung des Niederschlages weniger Nähr- und Schadstoffe im Boden gefiltert werden können.

Die Verkehrsinfrastruktur trägt massgeblich zur Versiegelung des Bodens bei aufgrund undurchlässigen Materials wie beispielsweise Teer oder Asphalt. Ebenfalls relevant, aber von geringerem Ausmass, sind die Anlagen zur Energieproduktion und -verteilung. Zwischen der treibstoffbedingten Mobilität und der Elektromobilität besteht in dieser Hinsicht jedoch kein grundsätzlicher Unterschied. Deshalb gibt es keine relevanten Auswirkungen.

\begin{tabular}{l|l|l|l}
\hline & & ICE & EV \\
\hline U3.2 & Bodenversiegelung reduzieren & $\approx$ & $\approx$ \\
\hline
\end{tabular}

\section{U3.3 Einsatz metallischer Rohstoffe minimieren}

Der Fahrzeugbau gehört zu den rohstoffintensiven Branchen. Neben den klassischen Metallen wie Stahl, Aluminium und Kupfer gewinnen die seltenen Metalle ständig an Bedeutung. Wie bereits im Kapitel 6 beschrieben, werden für die elektrischen Fahrzeuge viele seltene Rohstoffe genutzt. Neben der Menge an eingesetzten Rohstoffen spielt auch die Verfügbarkeit eine grosse Rolle. Der Aspekt der Verfügbarkeit der Ressourcen wird unter dem Teilziel W4.2 (Verfügbarkeit der Rohstoffe gewährleisten) diskutiert. 
Durch die stetige technische Innovation in der treibstoffbasierten individuellen Mobilität sowie der Elektromobilität kann der Einsatz der metallischen Rohstoffe möglicherweise in Zukunft reduziert werden. Für eine nachhaltige Nutzung der Rohstoffe sind die Rückgewinnung und das Recycling am Ende der Nutzungsdauer der Fahrzeuge oder einzelner Komponenten wie Batterien unerlässlich.

\begin{tabular}{|c|c|c|c|}
\hline & & ICE & EV \\
\hline U3.3 & $\begin{array}{l}\text { Einsatz metallischer Rohstoffe } \\
\text { minimieren }\end{array}$ & $+1-$ & $+/-$ \\
\hline
\end{tabular}

\section{U4 Natürliche Vielfalt fördern}

Die biologische Vielfalt stellt die Grundlage der Ernährung dar und liefert wichtige Baustoffe, Fasern und Medikamente. Für den Erhalt der Biodiversität müssen Lebensräume geschützt und die Zerschneidung von Landschaften reduziert werden.

\section{U4.1 Natürliche Lebensräume erhalten}

$\mathrm{Zu}$ betrachten sind einerseits der Bodenverbrauch für Infrastrukturanlagen und andererseits die Nutzung von natürlichen Ressourcen wie beispielsweise Boden, Biomasse oder Wasser für die Energieproduktion. Bezüglich Verkehrswegen und Infrastruktur zur Energieverteilung bestehen keine bedeutenden Unterschiede zwischen den beiden Mobilitätsarten, wie bereits im Abschnitt zur Bodenversiegelung ausgeführt wurde (U3.2).

Je nach Energieträger gibt es unterschiedliche Auswirkungen auf natürliche Lebensräume. In der Schweiz sind allfällige Beeinträchtigungen von natürlichen Lebensräumen bei einem verstärkten Ausbau der Elektromobilität möglich und bedingen eine sorgfältige Standortsuche für den Bau von Wasser- oder Windkraftwerken. Demgegenüber stehen jedoch die Auswirkungen durch den Verbrauch von fossilen Treibstoffen bei der treibstoffbedingten Mobilität. Die Erdölförderung ist je nach Technologie mit massiven Eingriffen in die Landschaft (insbesondere beim Abbau von Ölsanden) und mit erheblichen Risiken für die Ökosysteme verbunden. Auch die Produktion von Biotreibstoffen kann natürliche Lebensräume beeinträchtigen. Beispiele dazu sind die Abholzung von Wäldern für den Anbau von Ölpalmen oder die Intensivierung der Landwirtschaft für den Anbau von Energiepflanzen. 


\begin{tabular}{|c|c|c|c|}
\hline & & ICE & EV \\
\hline U4.1 & Natürliche Lebensräume erhalten & $+1-$ & +1 \\
\hline
\end{tabular}

\section{U4.2 Landschaftszerschneidung reduzieren}

Für den Erhalt der natürlichen Vielfalt ist der Austausch zwischen verschiedenen Populationen wichtig. Dieser wird durch die Zerschneidung von Landschaften durch Verkehrswege beeinträchtigt. Wie bereits beim Thema Bodenversiegelung (U3.2) erläutert, sind diesbezüglich keine Unterschiede zwischen der treibstoffbedingten Mobilität und Elektromobilität auszumachen.

\begin{tabular}{l|l|l|l}
\hline & & ICE & EV \\
\hline U4.2 & Landschaftszerschneidung reduzieren & $\approx$ & $\approx$ \\
\hline
\end{tabular}

\subsection{Wirtschaft}

\section{W1 Kosten minimieren}

Ziel ist es, die gegebenen Fahrleistungen wirtschaftlich und mit möglichst niedrigen Kosten zu erreichen. Dazu sollen die Investitions-, Betriebs- und Unterhaltskosten möglichst tief gehalten werden. Hierbei sind die Kosten der Fahrzeuge und die Kosten der Infrastruktur zu unterscheiden. Beide Aspekte werden hier berücksichtigt. Weitere volkswirtschaftliche Kosten, beispielsweise externe Kosten von Lärm oder Umweltwirkungen, sind bereits unter den Umweltzielen aufgeführt.

\section{W1.1 Investitions- und Betriebskosten für Fahrzeuge minimieren}

Die Kosten der Fahrzeuge setzen sich aus festen und variablen Kosten zusammen. Feste Kosten sind unabhängig von der Fahrleistung eines Fahrzeugs, variable Kosten sind abhängig von der Fahrleistung. Zunächst werden die Berechnungen für die beiden Kostenarten erläutert. Anschliessend werden die Gesamtkosten ausgewiesen. 
Die festen Kosten sind heute für ein Elektrofahrzeug deutlich höher als für ein treibstoffbetriebenes Fahrzeug. Bezüglich der batteriebetriebenen Fahrzeuge wird davon ausgegangen, dass der heutige Batteriepreis in Zukunft stark gesenkt werden kann. Dies vor allem infolge von Massenproduktion und Lerneffekten. Auch in Zukunft werden die Kosten für treibstoffbetriebene Fahrzeuge tendenziell tiefer liegen als bei Elektrofahrzeugen.

Hinsichtlich des Kaufverhaltens der Verbraucher ist festzuhalten, dass die Anschaffungsentscheide für ein Auto nur zu einem geringen Teil vom Kaufpreis abhängig sind. Status, Prestige und Wünsche an die Ausstattung führen heute schon zu erheblichen Preisunterschieden bei Fahrzeugen eines gleichen Modells. Es bedarf aber guter Argumente oder eines geschickten Ansprechens von Emotionen, um einen Käufer dazu zu bringen, für ein vergleichbares Fahrzeug mehr auszugeben.

Die variablen Kosten unterscheiden sich hauptsächlich bei den Energiekosten. Bei den treibstoffbedingten Fahrzeugen ist der Rohölpreis bestimmend, während bei den Elektrofahrzeugen der Elektrizitätspreis ausschlaggebend ist. Die Entwicklung der Weltmarktpreise für Rohöl ist von zahlreichen Faktoren abhängig, die heute nicht vorhersehbar sind. Basierend auf den heutigen Informationen zu Angebot und Nachfrage ist spätestens ab 2030 mit deutlichen Preissteigerungen zu rechnen. Aufgrund der aktuellen Energiedebatte in der Schweiz ist jedoch auch mit einem Anstieg der Elektrizitätspreise zu rechnen.

\begin{tabular}{c|l|c|c}
\hline & & ICE & EV \\
\hline W1.1 & $\begin{array}{l}\text { Investitions- und Betriebskosten für } \\
\text { Fahrzeuge minimieren }\end{array}$ & $!$ & $!$ \\
\hline
\end{tabular}

\section{W1.2 Investitions- und Betriebskosten für Infrastruktur minimieren}

Die Kosten der Infrastruktur beziehen sich einerseits auf die Verkehrswege und andererseits auf die Energieversorgung. Die Kosten für die Verkehrswege unterscheiden sich bei den beiden Mobilitätstypen nicht. Die Kosten für die Energieversorgung basieren auf den Erzeugungskosten der genutzten Energie wie beispielsweise der Kosten für die Rohölförderung oder die Elektrizitätsherstellung, die Verteilung sowie die Tankstellen und Ladestationen. Da für die treibstoffbedingte Mobilität die bestehenden Tankstellen genutzt werden können, sind bei der Elektromobilität grosse Investitions- und Installationskosten für Ladestationen nötig. Daher ist bei der Elektromobilität mit höheren Kosten zu rechnen. 


\begin{tabular}{l|l|c|c}
\hline W2.2 & $\begin{array}{l}\text { Einheimische Rohstoffe und Produkte } \\
\text { nutzen }\end{array}$ & $\approx$ & $(\checkmark)$ \\
\hline
\end{tabular}

\section{W2 Wertschöpfung erhöhen}

Quantitative, aber vor allem auch qualitative und langfristige Verbesserungen der ökonomischen Leistungsfähigkeit des Landes stehen bei diesem Zielbereich im Vordergrund. Die regionale Wertschöpfung soll durch die Nutzung einheimischer Energieträger und Produkte, aber auch durch den Aufbau einer wettbewerbsfähigen Exportwirtschaft in verschiedenen Stufen der Wertschöpfungskette erhöht werden.

\section{W2.1 Absatzmärkte für Schweizer Unternehmen schaffen}

Für Unternehmen bestehen im Kontext des Individualverkehrs zahlreiche Märkte, von der Herstellung von Fahrzeugkomponenten über die Herstellung oder Vermarktung von Treibstoffen bis zu Serviceleistungen.

Im Bereich der Fahrzeugherstellung sind Schweizer Unternehmen heute als Zulieferer tätig. Das Potenzial zur Erschliessung neuer Märkte ist dort am grössten, wo neue Produkte entwickelt werden und noch keine etablierten Marktstrukturen bestehen. Die Schweizer Wirtschaft zeichnet sich durch ein hohes Innovationspotenzial aus. Wichtige Voraussetzungen für die erfolgreiche Erschliessung von Marktpotenzialen sind Forschungs- und Entwicklungsaktivitäten sowie die Sicherstellung des Wissens- und Technologietransfers von den Hochschulen zu den Unternehmen. Wenn regulatorische Vorgaben einen schnelleren technologischen Wandel erzwingen, begünstigt dies prinzipiell die Exportchancen der Schweizer Zulieferer. Daher führen sowohl die verstärkte Förderung von Energieeffizienz von Verbrennungsmotoren als auch eine höhere Nachfrage nach Elektrofahrzeugen zu interessanten künftigen Absatzmärkten für Schweizer Unternehmer.

Schweizer Firmen sind heute namentlich im Bereich hochwertiger Komponenten und Werkstoffe tätig, wie auch bereits in verschiedenen Bereichen der Elektromobilität, von der Entwicklung von Ladestationen über Leistungselektronik bis hin zu Batteriemanagement, Fahrzeugkomponenten, Fahrzeugdesign sowie die Herstellung von Kleinserien. 
Die Energieversorgung für den Individualverkehr basiert heute primär auf Erdöl, einem importierten Energieträger. Bei einem Ausbau der Elektromobilität entstehen neue Absatzmärkte für die Schweizer Stromversorger. Weiter besteht bei der Entwicklung und Markteinführung von neuen Technologien die Möglichkeit zur Erschliessung neuer Märkte.

Zusammenfassend kommen wir zur Einschätzung, dass die Chancen zur Erschliessung neuer Absatzmärkte in der Elektromobilität gegenüber der treibstoffbasierten Mobilität steigen (wie wir in Kapitel 9.1.5 sehen werden, sind die Exportchancen im treibstoffbasierten EFF-Szenario aber ähnlich hoch wie für das COM-Szenario).

\begin{tabular}{l|l|c|c}
\hline W2.1 & $\begin{array}{l}\text { Absatzmärkte für Schweizer } \\
\text { Unternehmen schaffen }\end{array}$ & $\approx$ & ICE \\
\hline
\end{tabular}

\section{W2.2 Einheimische Rohstoffe und Produkte nutzen}

Die Rohstoffe für die Produktion von Fahrzeugen stammen überwiegend aus dem Ausland (vgl. U3.3). Betreffend der Nutzung der Primärenergien kann die Elektromobilität von einheimischem Strom profitieren, während die treibstoffbasierte Mobilität auch in Zukunft von Importen abhängig ist. Die Herkunft des Schweizer Stroms ist jedoch von mehreren Faktoren beeinflusst und hängt von der zukünftigen Energiestrategie des Bundesrates ab.

\begin{tabular}{|c|c|c|c|}
\hline & & ICE & EV \\
\hline W2.2 & $\begin{array}{l}\text { Einheimische Rohstoffe und Produkte } \\
\text { nutzen }\end{array}$ & $\approx$ & $(\sqrt{ })$ \\
\hline
\end{tabular}

\section{W3 Indirekte wirtschaftliche Effekte verbessern}

Durch die konzentrierte Schaffung und den Erhalt der wirtschaftlichen Tätigkeiten soll der Arbeitsstandort gestärkt werden. Forschung, Entwicklung und Technologie sind wichtige Voraussetzungen für die wirtschaftliche Leistungsfähigkeit. Auch regionale Zentren müssen davon profitieren können (dezentrale Konzentration). 


\section{W3.1 Unterstützung einer regional ausgeglichenen wirtschaftlichen Ent- wicklung}

Chancen für die wirtschaftliche Entwicklung ausserhalb der zentralen Wirtschaftsstandorte bieten sich durch die dezentrale Produktion von Strom für die Elektromobilität. Diese führt zu neuen Marktstrukturen und es sind neue Produkte und Dienstleistungen gefragt. Durch die Bildung von Technologie- und Wirtschaftsclustern in regionalen Zentren können auch Unternehmen ausserhalb der grossen Wirtschaftsregionen von den neuen Marktchancen profitieren.

\begin{tabular}{l|l|c|c}
\hline W3.1 & $\begin{array}{l}\text { Unterstützung einer regional } \\
\text { ausgeglichenen wirtschaftlichen } \\
\text { Entwicklung }\end{array}$ & $\approx$ & $(\checkmark)$ \\
\hline
\end{tabular}

\section{W3.3 Flexibilität des Systems erhöhen}

Ein flexibles System ermöglicht laufende Anpassungen an technologische Entwicklungen, sodass Effizienzfortschritte genutzt werden können. Flexibilität bedeutet den Verzicht auf grosse einmalige Investitionen und reduziert somit auch das Investitionsrisiko.

Ein weiterer Faktor der Flexibilität ist die dezentrale und auf verschiedenen Energieträgern basierende Energieversorgung (vgl. W4.1 Abhängigkeit von einzelnen Energieträgern senken). Die Abhängigkeit von einzelnen Energieträgern wird in der Elektromobilität gesenkt.

\begin{tabular}{l|l|c|c}
\hline W3.3 & $\begin{array}{l}\text { Flexibilität des Systems erhöhen } \\
\text { (Investitionsrisiko, Anpassungsfähigkeit) }\end{array}$ & $\approx$ & $(\checkmark)$ \\
\hline
\end{tabular}

\section{W4 Versorgungssicherheit gewährleisten}

Ein funktionierendes Mobilitätssystem ist eine wichtige Voraussetzung für eine leistungsfähige Wirtschaft und eine hohe Lebensqualität. Für die Gewährleistung der Mobilität sind die Energieversorgung und die notwendige Infrastruktur sicherzustellen. 


\section{W4.1 Abhängigkeit von einzelnen Energiequellen senken}

Gegenüber der treibstoffbasierten Mobilität entsteht bei der Elektromobilität eine Diversifizierung der Energieträger. Der Vorteil beim Einsatz von Strom ist, dass dieser Sekundärenergieträger aus verschiedenen Primärenergiequellen hergestellt werden kann. Damit sinkt die Abhängigkeit von einzelnen Energieträgern oder Versorgungsregionen. Ein weiterer Vorteil der Elektromobilität ist die Möglichkeit zum Einsatz erneuerbarer Energieträger. Diese sind zu einem grösseren Anteil im Inland vorhanden als bei flüssigen Treibstoffen, wodurch eine grössere Unabhängigkeit erreicht werden kann. Der Einsatz von Strom für Elektromobile erlaubt insbesondere die Verwendung stochastischer Energien und damit das Abschöpfung von Produktionsspitzen sowie das Vermeiden des Ladens zu Lastspitzen («Smart Charge»). Damit können Elektromobile, wenn richtig angesteuert, insbesondere einen Beitrag leisten zu stabileren lokalen Stromnetzen auf der untersten Netzebene; ohne eine intelligente Ansteuerung kann jedoch auch der gegenteilige Effekt eintreten.

\begin{tabular}{l|c|c|c}
\hline W4.1 & $\begin{array}{l}\text { Abhängigkeit von einzelnen } \\
\text { Energieträgern senken }\end{array}$ & ICE & EV \\
\hline
\end{tabular}

\section{W4.2 Verfügbarkeit der Rohstoffe gewährleisten}

In den Mobilitätstypen kommen Antriebs- und Energiespeicherkonzepte zum Einsatz, die aus unterschiedlichen Rohstoffen hergestellt werden. Die Verfügbarkeit dieser Rohstoffe ist einerseits eine Voraussetzung für die Verbreitung der jeweiligen Technologien. Andererseits ist auch der Einsatz für andere Anwendungen zu gewährleisten. Die Verfügbarkeit der kritischen Rohstoffe wurde im Kapitel 6 erläutert.

In der zusammenfassenden Beurteilung der Auswirkungen zeigt sich kein einheitliches Bild. Die Ausgangslage ist bei jedem einzelnen Rohstoff etwas anders. Grundsätzlich sind ausreichende Reserven vorhanden. Bei allen Mobilitätstypen werden jedoch Rohstoffe eingesetzt, deren Verfügbarkeit durch Exportbeschränkungen problematisch sein kann. Diese geopolitischen Risiken sind für die Schweiz als rohstoffarmes Land schwierig einschätzbar. Engpässe in der Fahrzeugproduktion würden die schweizerische Volkswirtschaft nur mit Verzögerung beeinträchtigen, weil die bestehenden Fahrzeuge weiterhin betrieben werden 
können. Versorgungsengpässe bei fossilen Treibstoffen hätten hingegen einen unmittelbaren Effekt.

\begin{tabular}{l|c|c|c}
\hline W4.2 & $\begin{array}{l}\text { Verfügbarkeit der Rohstoffe } \\
\text { gewährleisten }\end{array}$ & ICE & EV \\
\hline
\end{tabular}

\subsection{Gesellschaft}

\section{G1 Gesundheit und Wohlbefinden fördern}

Der physische und psychische Gesundheitszustand beeinflusst in hohem Masse die Lebensqualität und ist ein wichtiger Faktor für die wirtschaftliche Leistungsfähigkeit der Bevölkerung. Die Möglichkeiten der individuellen Mobilität werden positiv erlebt, viele Auswirkungen des Verkehrs jedoch negativ. Die Zahl der Unfallopfer und der durch den Verkehr in ihrer Gesundheit beeinträchtigten Personen soll verringert werden. Dazu müssen die Verkehrssicherheit erhöht und die Störfallrisiken minimiert werden. Als weiterer Beitrag zum Wohlbefinden sollen Verkehrs- und Energieversorgungsinfrastrukturen die Qualität von Siedlungs- und Landschaftsbildern möglichst wenig beeinträchtigen.

\section{G1.1 Verkehrssicherheit erhöhen}

Unfälle mit Todesfolgen und/oder schwerwiegenden Verletzungen sind in fast allen industrialisierten Ländern rückläufig. In der Schweiz ist die Anzahl von im Strassenverkehr schwerverunfallter (schwerverletzter und getöteter) Personen im vergangenen Jahrzehnt um ein Viertel gesunken: 2009 wurden verglichen mit 200041 Prozent weniger Personen im Strassenverkehr getötet, 24 Prozent weniger schwer verletzt und 14 Prozent weniger leicht verletzt (BFS 2010a). Der Rückgang ist am grössten bei Insassen von Personenwagen; bei den Motorrädern ist die Zahl der Verunfallten gestiegen.

Wichtige Einflussfaktoren auf die Verkehrssicherheit und damit Ansatzpunkte für Verbesserungen sind das Verhalten der Fahrzeuglenkenden, die Fahrleistungen insgesamt, die Voraussetzungen bezüglich Fahrzeugen und Infrastruktur sowie vermehrt auch der Einsatz von Technologien zur Unfallvermeidung wie beispielsweise automatische Abstandsmessung und elektronische Steuerung. Für 
Mobilitätstypen gilt, dass die positive Entwicklung bei den Unfällen mit Personenwagen nur fortgesetzt werden kann, wenn die bisherigen Anstrengungen weitergeführt und ausgebaut werden. Zudem sind neue Entwicklungen sowohl gesellschaftlicher als auch technischer Art frühzeitig zu erkennen, damit bei Bedarf geeignete Massnahmen in die Wege geleitetet werden können.

Unabhängig von der Antriebstechnologie werden schon heute vermehrt elektronische Systeme eingesetzt, die unterstützend wirken und das Unfallrisiko minimieren bzw. den Unfall vermeiden helfen («aktive Sicherheit»). Dieser Trend wird sich fortsetzen. Gerade bei kleineren und leichteren Elektrofahrzeugen tragen aktive Sicherheitssysteme künftig einen wichtigen Teil zur Fahrzeugsicherheit bei, ohne dabei das Gewicht des Fahrzeugs massgeblich zu erhöhen. Elektronische Stabilitätsprogramme, Fussgängerfrüherkennung und andere Fahrassistenten sind Sicherheitssysteme, die helfen, dass es gar nicht erst zu einem Zusammenstoss kommen kann. Der gegenwärtige «eSafety-Trend» in der Fahrzeugsicherheit und die Nachfrage nach Systemen der Unfallvermeidung würde durch die Einführung von Elektroautos wohl verstärkt, weil diese für Elektroautos sehr wesentliche Beiträge zur Sicherheit liefern können. Bis ins Jahr 2050 werden diese Systeme jedoch unabhängig von der Antriebstechnologie verbreitet eingesetzt werden. Zu bedenken ist allerdings, dass von einzelnen (OEM-basierten) Systemen hin zu einer übersteuernden oder sogar automatisierten Fahrweise ein langer Weg ist. Die passive Sicherheit wird deshalb auch langfristig mit grosser Wahrscheinlichkeit das tragende Sicherheitselement darstellen.

Der Vergleich der verschiedenen Mobilitätstypen gibt Hinweise darauf, dass die heute in Kleinserien hergestellten Elektroautos bezüglich Sicherheitsstandards nicht die gleichen Anforderungen erfüllen wie vergleichbare Micro- oder Kleinwagen mit Verbrennungsmotor. Aus den bisherigen Erfahrungen und wegen teilweise fehlender Sicherheitssysteme bei heute verfügbaren Modellen von Elektroautos kann jedoch nicht auf die Sicherheit von elektrisch angetriebenen Autos generell geschlossen werden. Die technischen Spezifikationen von Elektroautos und veränderte Anforderungen an die Konstruktion können sich positiv auf Sicherheitsaspekte auswirken. Ein Beispiel ist die fehlende Verbrennungsmaschine unter der Motorhaube. Anstatt des Motorblocks und des Getriebes befindet sich beim Stromauto dort der wesentliche kleinere Elektromotor, wenn er nicht sogar im Heck oder in den Radnaben untergebracht ist, wie im Falle eines Prototyps der Firma Michelin. Diese Veränderung ermöglicht eine gänzlich andere Frontgestaltung, welche die Chance bietet, das Risiko einer schweren Kopfverletzung im Falle einer Kollision mit einem Fussgänger weiter zu senken. 
Ein weiterer Einflussfaktor ist der reduzierte Lärmpegel bei niedrigen Geschwindigkeiten. Vor allem schwächere Verkehrsteilnehmer orientieren sich oftmals nach Gehör, und fehlender Motorenlärm birgt somit ein gewisses Gefahrenpotenzial. Ein potenzielles Risiko lässt sich insbesondere für seh- und hörgeschwächte Verkehrsteilnehmer ableiten. In den Vereinigten Staaten wird deshalb aktuell über die Anbringung von akustischen Signalen an Hybridfahrzeuge diskutier $t^{9}$ ) und in Japan wurde auf den 1. Januar 2010 bereits eine Richtlinie über Massnahmen zur besseren Hörbarkeit von Hybridfahrzeugen in Kraft gesetzt ${ }^{10}$ ). So kann dieses mit Elektrofahrzeugen einhergehende neue Unfallrisiko mit einfachen Mitteln reduziert werden. Bei höheren Geschwindigkeiten (> $30 \mathrm{~km} / \mathrm{h}$ ) existiert dieses Problem aufgrund der dann dominierenden Roll- und Windgeräusche nicht mehr. Zu berücksichtigen ist zudem, dass die Orientierung per Ohr je nach räumlichem Kontext und Verkehrsdichte eine unterschiedliche Rolle spielt. Gerade in weniger stark befahrenen und Wohnquartieren spielt die "Ohrientierung» neben der Orientierung per Auge eine wichtige Rolle, werden Motorfahrzeuge doch hier aufgrund des leiseren Umfelds oftmals gehört, bevor sie gesehen werden. Im dichten lauten Stadtverkehr hingegen dürfte die "Ohrientierung» eher eine untergeordnete Rolle spielen.

Andere Einflüsse der Elektromobilität ergeben sich durch reduzierte Durchschnittsgeschwindigkeiten bei wachsenden Elektrifizierungsgraden und damit sinkende Unfällen infolge von Fahrten mit stark überhöhten Geschwindigkeiten («Raserunfälle»). Gegenüber einem thermischen Motor erlaubt der Elektroantrieb im Regelbetrieb Maximalgeschwindigkeiten, die noch deutlich unterhalb jener liegen, die sich mit den heutigen Personenwagen erreichen lassen. Bei Zweirädern führt die Elektromobilität jedoch zu höheren mittleren Geschwindigkeiten und damit zu einer möglichen Zunahme von Unfällen. Dies hat bereits erste regulatorische Massnamen erfordert wie beispielsweise die Unterteilung in Kategorien nach Maximalgeschwindigkeit, die Helmpflicht oder die Einstufung als Motorräder.

\begin{tabular}{l|l|c|c}
\hline & & ICE & EV \\
\hline G1.1 & Verkehrssicherheit erhöhen & & $\checkmark$ \\
\hline
\end{tabular}

9 Vgl. z.B. Studien zur Wahrnehmung von Fahrzeugen http://newsroom.ucr.edu/news item.html?action=page\&id=1803 oder "Toyota baut Krachmacher ein», http://www.n-tv. de/auto/Toyota-baut-Krachmacher-ein-article1345616.html.

10 "Guideline on measures against the quietness of hybrid vehicles, etc.» Die Richtlinie ist eine Empfehlung der japanischen Behörde an die Fahrzeughersteller. 


\section{G1.2 Verringerung von Störfallrisiken}

Während unter G1.1 «Verkehrssicherheit erhöhen» der Betrieb der Fahrzeuge berücksichtigt wird, geht es hier um Risiken im Zusammenhang mit dem Treibstoff oder der Antriebstechnologie. Dabei werden die Bereiche Produktion, Lagerung und Transport von Treibstoffen sowie Tankstellen und der Einsatz der Treibstoffe in Fahrzeugen berücksichtigt.

Mit dem Einsatz der heute gängigen Treibstoffe Benzin und Diesel ist nur ein geringes Todesfallrisiko verbunden. Der Anteil des treibstoffbedingten Risikos am gesamten kollektiven Todesfallrisiko im Strassenverkehr liegt unter einem Prozent (EBP 2009). Im Vordergrund stehen dabei Risiken durch schwere Verkehrsunfälle beim Treibstofftransport. Die Risiken bei Lagerung und Transport der Treibstoffe sind marginal. Ausserhalb der Schweiz, für die Bevölkerung an den Produktionsstätten, bestehen lokal teilweise hohe Risiken (wie beispielsweise die Explosionsgefahr einer Raffinerie oder Pipeline) oder gesundheitliche Auswirkungen.

Bei der Elektromobilität ist das Risiko von der Stromproduktion abhängig. Da die Produktion grösstenteils in der Schweiz oder im näheren Ausland stattfindet, ist die Schweiz von den Risiken direkter betroffen als bei fossilen Treibstoffen. Die verschiedenen Stromproduktionsverfahren unterscheiden sich stark: Bei neueren Technologien wie Fotovoltaik- oder Biogasanlagen ist häufiger mit Störfällen, aber mit geringen Schäden zu rechnen. Bei der Kernkraft hingegen ist die Wahrscheinlichkeit von Störfällen tiefer, der potenzielle Schaden dafür bedeutend höher.

Bei der Lagerung und Verteilung von Treibstoffen ist das Risiko von der Speicher- und Treibstoffart abhängig. Flüssige, herkömmliche Treibstoffe wie Diesel oder Benzin sind potenzielle Gefahren für das Grund- und Oberflächenwasser und die umliegende Natur. Zudem besteht das Risiko einer Explosion oder schwerwiegender Grossbrände. Für die Elektromobilität besteht hier ein nur geringes Risiko, da der Strom nicht auf Vorrat produziert, sondern direkt eingespeist wird.

Das Risiko im Betrieb der Fahrzeuge ist von der Art des Treibstoffs sowie von der Speicher- und Antriebstechnologie abhängig. Beim Einsatz von Elektromotoren mit hohen Spannungen kann es zu Stromschlägen kommen sowie zu Überhitzungen und als Resultat zu Bränden. Des Weiteren geht Gefahr von austreten- 
den Batterieelektrolyten aus. Das grösste Risiko bei Benzin und Diesel besteht, im Falle eines Treibstoffaustritts, aber auch bei der Lagerung, in der lokalen Verschmutzung der Umwelt und der Entstehung von Bränden.

Bei Rettungseinsätzen an verunfallten Fahrzeugen stellen Treibstofftanks grosse Risiken dar; beim häufigsten Fall des Fahrzeugbrandes verbleiben den Rettern jedoch wertvolle Sekunden. Hohe Spannungen (bei einem verunfallten Fahrzeug mit Elektrobatterie) sind deshalb gefährlich, weil sie nicht wahrgenommen werden können. Heutige Hybrid- und Elektroautos sind jedoch bereits so konstruiert, dass die hohen Spannungen nur in wenigen Bauteilen unmittelbar bei der Batterie vorkommen und im Falle einer Kollision von den übrigen Fahrzeugsystemen getrennt werden.

\begin{tabular}{l|c|c|c}
\hline & & ICE & EV \\
\hline G1.2 & Minimierung von Störfallrisiken & $\approx$ & $+/-$ \\
\hline
\end{tabular}

\section{G1.3 Beeinträchtigungen des Siedlungs- und Landschaftsbildes minimie- ren}

Die Auswirkungen auf das Siedlungs- und Landschaftsbild sind verknüpft mit der Bodenversiegelung (U3) und der Biodiversität (U4).

Von entscheidender Bedeutung für je nach Zukunftsbild unterschiedliche Auswirkungen auf das Landschaftsbild ist, welche Treibstoffe eingesetzt und wie diese hergestellt werden. Bei fossilen Energieträgern entsteht die grösste Beeinträchtigung bei der Rohölförderung. Bei der Elektromobilität ist die Stromproduktion relevant. Diese ist bisher eher lokal, mit Auswirkungen auf das Landschaftsbild durch grosse zentrale oder viele kleinere dezentrale Anlagen. Mit Projekten wie Desertec werden aber auch hier die mit der Produktion verbundenen negativen Auswirkungen ins Ausland verschoben.

Das Siedlungsbild wird von der Betankungsinfrastruktur beeinflusst. Dies hängt in beiden Mobilitätstypen von der Umsetzung ab. Bei der Elektromobilität können beispielsweise bestehende Tankstellen rückgebaut werden, doch im Gegenzug werden Ladestationen im öffentlichen Raum gebaut. Wie stark diese das Siedlungsbild beeinflussen und ob sie als negativ oder positiv wahrgenommen werden, ist von ihrer Gestaltung abhängig. 


\begin{tabular}{l|l|c|c}
\hline G1.3 & $\begin{array}{l}\text { Beeinträchtigungen des Siedlungs- und } \\
\text { Landschaftsbildes minimieren }\end{array}$ & $+/-$ & $+/-$ \\
\hline
\end{tabular}

\section{G2 Gesellschaftliche Solidarität fördern}

Im Bereich der Mobilität ist die Berücksichtigung der unterschiedlichen Verhalten und Mobilitätsbedürfnisse verschiedener sozialer und gesellschaftlicher Gruppen wichtig. Es sollen dabei besonders Menschen mit einem erschwerten Zugang zu Verkehrsinfrastruktur und alle Formen der Mobilität berücksichtigt werden. Von Bedeutung ist auch eine gute Anbindung ländlicher Räume.

\section{G2.1 Zugang für alle Regionen und gesellschaftlichen Gruppen sicherstellen}

Zugang zur Mobilität bedeutet die Möglichkeit, sich im geografischen Raum zu bewegen. Voraussetzungen dafür sind einerseits die Verfügbarkeit von Transportmitteln und Verkehrsinfrastruktur und andererseits die persönlichen Möglichkeiten, die für die Nutzung dieser Transportmittel notwendig sind. Die Solidarität ist beeinträchtigt, wenn diesbezüglich grosse Unterschiede zwischen Regionen, verschiedenen Verkehrsteilnehmern oder gesellschaftlichen Gruppen bestehen. Bezüglich der Verkehrsinfrastruktur unterscheiden sich die Mobilitätstypen kaum. Relevant sind aber die Unterschiede bei der Infrastruktur zur Energieverteilung. Für die Versorgung der Elektromobilität in allen Regionen ist ein flächendeckender Ausbau der Elektromobilitätsinfrastruktur notwendig. Auch in städtischen Gebieten ist zumindest in der Übergangsphase eine Benachteiligung von Fahrzeughaltern möglich, die über keine eigene Garage oder keinen Abstellplatz mit Lademöglichkeit verfügen. Diese Herausforderungen sind jedoch insgesamt gering, namentlich im Vergleich zu anderen alternativen Treibstoffen wie Gas oder Wasserstoff, weil das Stromnetz in der Schweiz flächendeckend vorhanden ist.

\begin{tabular}{l|l|c|c}
\hline G2.1 & $\begin{array}{l}\text { Zugang für alle Regionen und } \\
\text { gesellschaftlichen Gruppen sicherstellen }\end{array}$ & $\approx$ & $\approx$ \\
\hline
\end{tabular}




\section{$8 \quad$ Reboundeffekte}

\subsection{Treiber für Reboundeffekte}

Die meisten Treiber für motorisierte individuelle Mobilität sind extern (Bevölkerungswachstum, Wohlstand, Verstädterung, ÖV-Angebot, Haushaltstrukturen, Verkehrsinfrastruktur) und haben nichts mit Eigenschaften von bestimmten Antriebstechnologien zu tun. Um keine Äpfel mit Birnen zu vergleichen, werden deshalb im vorliegenden Bericht alle Szenarien für die gleiche Anzahl Personenund Fahrzeugkilometer durchgespielt.

Es stellt sich die Frage, ob es Eigenschaften bestimmter Antriebstechnologien gibt, welche für sich genommen wieder Rückkopplungen auf das Mobilitätsverhalten und damit auf die Anzahl Fahrzeug- und Personenkilometer haben könnten. Fährt man mehr, nachdem man zum Beispiel von einem VW Golf auf ein Hybridfahrzeug gewechselt hat?

Für Elektrofahrzeuge liegen dazu noch keine empirischen Studien vor, weil es noch wenige Elektrofahrzeugkäufer gibt. Für Hybridfahrzeuge wurde dies empirisch untersucht. Reboundeffekte wurden jedoch keine festgestellt (de Haan et al. 2006a, 2006b, 2007). Es ist aber denkbar, dass Elektro- und Hybridfahrzeuge anfangs von Early-Market-Käufergruppen erworben werden, bei denen weniger Reboundeffekte auftreten als später im Massenmarkt. Dies untersuchen wir im Folgenden qualitativ.

Reboundeffekte bezeichnen eine zusätzliche Nachfrage nach einer Dienstleistung oder einem Produkt aufgrund höherer Effizienz. Damit die höhere Effizienz ein Nachfragesignal auslöst, muss eine gewisse von Null verschiedene Elastizität vorhanden sein, die Effizienz also ein knappes Gut betreffen. Reboundeffekte sind deshalb ganz allgemein Ressourceneffizienzeffekte. Es kann sich sowohl um Zeitressourcen handeln (Umfahrungsstrassen führen zu höherer Zeiteffizienz und deshalb zu Mehrverkehr), um Raumressourcen (erhöhte Raumeffizienz führt zu erhöhter Nachfrage nach raumrelevanten Dienstleistungen, erhöhen Städte die Ausnützungsziffern, ziehen mehr Personen in die Stadt), um Energieressourcen (der Umstieg vom ÖV auf das neue, sehr energieeffiziente Auto) oder um andere physikalische Ressourcen. 
Kein Reboundeffekt liegt hingegen vor, wenn die Nachfrage nach einem Produkt steigt, weil die Kaufkraft gestiegen ist oder sich Konsumentenpräferenzen geändert haben. Die oft angestellten makroökonomischen Vergleiche, bei denen die gesamte Mehrnachfrage über einen längeren Zeitraum in Relation gesetzt wird zur gesamten Effizienzsteigerung, um daraus zu folgern, dass Effizienzsteigerungen keinen Effekt hätten, sind nicht zutreffend. Während sich zum Beispiel im Zeitraum von 1960 bis 1995 in Deutschland der mittlere Verbrauch halbierte, verdoppelte sich der Verkehr - es gibt Studien, die daraus zu schliessen versuchen, dass sparsamere Autos zu mehr Autokilometer führen. Solche Analysen ignorieren den grundlegenden Einfluss des wirtschaftlichen Wachstums. Gemäss vielen Untersuchungen (z.B. Gasche und Guggenbühl 2010) ist das wirtschaftliche Wachstum mit dem Verbrauch der Primärenergie gekoppelt. Je grösser die Wirtschaftsleistung, desto grösser ist der Verbrauch von Primärenergie und die damit verbundenen negativen Einflüsse auf Umwelt, Natur und Klima. Mit Reboundeffekten hat dies nichts zu tun. Auch die Nachfrage nach vielen Produkten, deren Herstellung nicht energieeffizienter geworden ist (z.B. Fleisch), nimmt in Zeiten von Wirtschaftswachstum zu.

\subsection{Können effiziente Fahrzeuge Reboundeffekte induzieren?}

Wir betrachten im Folgenden nur Reboundeffekte aufgrund effizienterer Fahrzeuge (egal ob elektrisch oder mit Verbrennungsmotor angetrieben). Für die Analyse von Reboundeffekten aufgrund höherer Zeiteffizienz verweisen wir auf Spielmann et al. (2008) und gehen hier davon aus, dass effiziente Fahrzeuge gleich schnell unterwegs sind wie der Durchschnitt.

Es darf davon ausgegangen werden, dass Reboundeffekte im Rahmen des Mobilitätsdiskurses zwar von hoher Bedeutung sind, dass die Herausforderungen aber in den meisten Fällen nicht spezifisch mit der Elektromobilität zusammenhängen, sondern generell mit effizienten Antrieben. Wo der elektrische Antrieb eine spezifische Rolle spielt, wird dies vermerkt.

Reboundeffekte aus finanziellen Gründen: Effizientere Fahrzeuge führen zu geringeren Treibstoffkosten. Mit dem eingesparten Geld lässt sich ein grösseres Auto kaufen oder man ist öfter unterwegs oder konsumiert andere (ebenfalls mit Energieaufwand verbundene) Produkte. 
- Höhere Effizienz beim Auto kann über zwei Pfade erreicht werden: Aufwendigere Technik oder «weniger Auto». Hybridtechnologie und Leichtbaumaterialien sind Beispiele für den ersten Pfad. Die aufwendigere Technik führt zu einem höheren Kaufpreis, was (bei heutigen Energiepreisen) die Kosteneinsparungen beim Betrieb weitgehend kompensiert. Über die ganze Lebensdauer gesehen, kommt es also kaum zu Kosteneinsparungen und damit nur zu einem geringen oder keinem Reboundeffekt. Der andere Effizienzpfad heisst «kleineres Auto» und/oder «Motor mit weniger Leistungsreserven». Hierfür ist keine aufwendigere Technik nötig, solche Autos sind erst noch günstiger im Erwerb als die grösseren bzw. leistungsstärkeren Schwestermodelle. Der Kunde hat also mehr Geld zur Verfügung. Wechselt der Kunde freiwillig zu «weniger Auto», stehen geänderte Präferenzen im Vordergrund, nicht eine effizientere Technologie - dies ist dann kein Reboundeffekt. Erzwingt aber der Staat (zum Beispiel über Vorschriften für Neuwagenzulassungen) den Wechsel hin zu «weniger Auto», sind Reboundeffekte denkbar.

- Die marginalen Kosten pro Fahrzeugkilometer sind für effiziente Fahrzeuge jedoch geringer, ganz speziell für Elektrofahrzeuge, solang der Strom im Gegensatz zu Benzin nicht besteuert wird. Dem steht gegenüber, dass die Batterie nicht nur kalendarisch altert, sondern auch mit jedem Entlade-Lade-Zyklus. Welcher Alterungsprozess dominiert, hängt vom Batterietyp ab, und auch davon, ob das Fahrzeug eher wenig oder eher mehr gefahren wird. Je nach Batterietyp und Einsatzart sind damit die marginalen Kosten unterschiedlich und damit ein finanzieller Treiber für mögliche finanzielle Reboundeffekte vorhanden oder auch nicht.

- Mittelfristig sollte der Staat die Mineralölsteuer erhöhen und damit die Preissignale aus der höheren Effizienz konventioneller Autos möglichst abschöpfen (setzt sich der Staat zum Ziel, konstante Steuereinnahmen zu generieren, geschieht diese Abschöpfung quasi automatisch in der richtigen Höhe). Natürlich wird die öffentliche Debatte hier über die Erträge und deren Verwendung geführt, dennoch ist eine solche Erhöhung schon rein aus Sicht der Vermeidung von Reboundeffekten sinnvoll.

- Längerfristig (je früher die Elektromobilität in den Massenmarkt eintritt, desto eher) sollte von der Mineralölsteuer auf eine vom Energieträger unabhängige, kilometerbasierte Besteuerung («Road Pricing» oder ein auch den öffentlichen Verkehr umfassendes «Mobility Pricing») umgestellt werden, welches idealerweise auch von der Primärenergieeffizienz abhängig wäre. Diese Ab- 
hängigkeit auch von der Primarenergie bewirkt, dass der Systemwechsel früher eingeführt werden kann, ohne dass deswegen die Markteindringung der Elektromobilität gefährdet würde.

Generell lässt sich sagen, dass die Abschöpfung der Effizienzgewinne über höhere Energiesteuern (sei dies nun eine Mineralölsteuer, eine $\mathrm{CO}_{2}$-Steuer oder eine kilometerbasierte Besteuerung) dem finanziellen Rebound effektiv entgegentritt. Es gibt hier Zielsynergien mit dem allgemeinen Bestreben nach einer weiteren Internalisierung der externen Kosten, namentlich im Bereich der Mobilität. Die Frage des Anteils der Elektromobilität ist in diesem Zusammenhang zweitrangig; allenfalls lässt sich argumentieren, dass mehr Elektromobilität schneller zur Einführung einer kilometerabhängigen Besteuerung führt und damit ein wirksames Instrument zur Eindämmung finanzieller Reboundeffekte im Bereich der Mobilität darstellt. Elektromobilität kann aber auch zu mentalen Reboundeffekten führen, siehe nachstehend.

Reboundeffekte aus mentalen (sozialpsychologischen) Gründen: Neben dem finanziellen Effekt spielt auch der sozialpsychologische eine Rolle, namentlich bei der Mobilität. Oft sind nicht die Kosten an sich der hemmende Faktor, sondern die wahrgenommene Umweltbelastung. Das Konzept des «mentalen Rebound» (de Haan 2009; Girod und de Haan 2010; Girod et al. 2010) geht davon aus, dass Konsumenten verschiedene «mentale Budgets» haben, darunter auch eines für «durch Autofahren verursachte Umweltbelastung». Es liegt meist nicht am Geld, wenn Konsumenten in der Schweiz die Absicht äussern, weniger Autokilometer zu fahren.

Aus dieser Optik weist Elektromobilität ein hohes Reboundpotenzial auf: Es reduziert erstens die (finanziellen) Energiekosten und zweitens die (mentalen) Kosten des Einsatzes von Motorfahrzeugen. Weil die Energiekosten mental meist stärker wahrgenommen werden als die Anschaffungs- und Unterhaltskosten, können scheinbar finanziell begründete Reboundeffekte auftreten, auch wenn es sie rechnerisch nicht gibt. Die reduzierten mentalen Kosten können dazu führen, dass Langsamverkehr (Zu-Fuss-Gehen, Fahrrad) und ÖV durch Elektromobile substituiert werden.

Neben finanziellen und mentalen Reboundeffekten kennt die Literatur auch Reboundeffekte aus regulatorischen Gründen. Sollte die Energiepolitik im Bereich des MIV auch längerfristig stark auf den mittleren $\mathrm{g} \mathrm{CO}_{2} / \mathrm{km}$-Wert der PKW-Neuzu- 
lassungen abstützen und sollte dabei für Elektrofahrzeuge eine sehr niedrige $\mathrm{CO}_{2}$ Intensität des zugrunde gelegten Strommixes angenommen werden, resultiert dies (i) zu einem Förderinstrument für die vermehrte Neuzulassung von Elektrofahrzeugen; (ii) zur Förderung auch ineffizienter Elektrofahrzeuge; (iii) zu einer Aufweichung des mittleren $\mathrm{g} \mathrm{CO}_{2} / \mathrm{km}$-Teilziels für Fahrzeuge mit Verbrennungsmotor.

\subsection{Massnahmen zur Reduktion von Rebound- effekten}

Die Förderung einer energieeffizienten Mobilität führt in einem ersten Schritt dazu, dass die Treibstoffkosten der Autofahrenden sinken. Man könnte deshalb das Auftreten eines direkten Reboundeffekts aus finanziellen Gründen postulieren. Allerdings stehen dem die folgenden beiden Aspekte entgegen:

- Wenn der Staat das längerfristige Ziel einer mindestens gleichbleibenden Besteuerung des MIV verfolgt, um Unterhalt und weiteren Ausbau des Strassennetzes zu finanzieren, ist ein pro Fahrzeug in etwa konstanter Steuerertrag notwendig. Ob nun die Mineralölsteuer erhöht wird oder neue Ansätze wie eine kilometerabhängige Besteuerung verfolgt werden, das Ergebnis ist identisch: die nahezu vollständige Kompensation der finanziellen Effekte der Effizienzgewinne. Der Anteil des Treibstoffs an den Gesamtkosten eines Durchschnittsautos betrug 2011 in der Schweiz 14,9 Prozent, davon entfällt rund ein Drittel auf den (vom Weltmarkt abhängigen) Preis für den Treibstoff selber, zwei Drittel sind Steuern und Abgaben. Wenn nun also der Treibstoffverbrauch sich um 50 Prozent reduziert, nehmen die Kosten des Durchschnittswagen nur um 2,5 Prozent ab. Und auch dies gilt nur, wenn die höhere Treibstoffeffizienz keine aufwendigere Technik und damit einen höheren Kaufpreis für das Auto erfordert hat.

- Die individuelle motorisierte Mobilität ist eher zeit- als kostenlimitiert. Die Zeitkosten für das Zurücklegen eines Kilometers mit dem Auto sind sogar etwas höher als die Autokosten: Mit einem mittleren Zeitkostenansatz von 24,1 Franken pro Person (EBP 2008) und einem mittleren Belegungsgrad von 1,6 (BFS 2012) belaufen sich die Zeitkosten auf 38,6 Franken pro Auto und Stunde. Die Autokosten errechnen sich aus mittleren 0,75 Franken pro Kilometer (TCS 2012) und einer mittleren Geschwindigkeit von 38,6 Stundenkilometer (BFS 
2012) zu 29,0 Franken pro Auto und Stunde. Deshalb sind direkte Reboundeffekte im Bereich des MIV durch die damit einhergehenden Zeitkosten limitiert. Es stehen deshalb indirekte Reboundeffekte im Vordergrund.

Nur energieeffiziente Elektroautos sind ein sinnvoller Beitrag zu einer nachhaltigen Mobilität und damit letztlich auch im Interesse der Elektromobilität selber. Deshalb erscheint es nicht angezeigt, die Besteuerung der Elektromobilität hinauszuzögern mit dem Ziel, damit die Marktdurchdringung der Elektromobilität zu beschleunigen. Diese Beschleunigung muss mit anderen Mitteln erreicht werden, welche generell die Gesamtenergieeffizienz des MIV anpeilen. Eine technologiespezifische Förderung (z.B. durch andauernden Verzicht auf eine Besteuerung der Elektromobilität) kann zu falschen Signalen führen (z.B. würden damit ineffiziente Elektrofahrzeuge gefördert und Fahrzeuge mit hocheffizienten Verbrennungsmotoren, relativ gesehen, bestraft). Die Einführung einer auch die Elektromobilität umfassenden und die Mineralölsteuer ersetzenden kilometerabhängigen Besteuerung, welche aus Sicht der Elektromobilität idealerweise zusätzlich von der Primärenergieeffizienz abhängig wäre, ist deshalb zu befürworten und würde ein wirksames Mittel zur Eindämmung von finanziellen Reboundeffekten im Zusammenhang mit der Elektromobilität darstellen.

Die grössten Treiber für Reboundeffekte im Zusammenhang mit elektrischen Antrieben sind damit nicht finanzieller oder sozialpsychologischer (mentaler) Art. Elektrofahrzeuge sind gute Stadtfahrzeuge; damit treten sie aber auch in Konkurrenz zu städtischen ÖV-Systemen. Elektrofahrzeuge können auch zum Langsamverkehr in Konkurrenz treten, namentlich zu heute noch antriebslosen Fahrrädern. Auch diesen Reboundeffekten kann nicht mit technologiespezifischen Massnahmen entgegengewirkt werden, vielmehr stehen hier weiterhin die Parkplatzbewirtschaftung, die Reduzierung der Pflichtparkplätze, die Verdichtung im urbanen Raum usw. als Massnahmen im Vordergrund. Längerfristig führt kein Weg vorbei an der Eindämmung des mentalen Reboundeffekts über (a) Umweltbildung (wenn die Konsumenten besser Bescheid wissen über die Umweltauswirkungen des MIV, werden sie auch nach dem Umstieg auf effizientere Fahrzeuge weiterhin versuchen, diese Auswirkungen zu reduzieren) und (b) die Schaffung neuer Normen durch Beeinflussung von "Opinion Leaders» und das Setzen entsprechender Anreizsysteme und Verbote. Hier können namentlich die effizienzabhängige Ausgestaltung von Pendelkostenabzügen und der Anrechnung der privaten Nutzung von Dienstfahrzeugen im Lohnausweis eine grössere Wirkung entfalten. 


\section{Gesamtbeurteilung, Fazit und Empfehlung}

\subsection{Resultate für alle Szenarien}

\subsubsection{Technologieentwicklung}

Bei allen Fahrzeugen erwarten wir in den nächsten Jahren und Jahrzehnten eine substanzielle Erhöhung des energetischen Wirkungsgrades und eine Reduktion der Fahrzeugmasse bei gleichbleibender Fahrzeuggrösse. Die Massenreduktion wird erreicht durch die Verwendung spezifisch leichterer Materialien wie hochfestem Stahl, Aluminium oder faserverstärkten Kunststoffen. Während die Wirkungsgraderhöhung bei ICE-Fahrzeugen beträchtlich sein dürfte, erwarten wir bei den elektrischen Fahrzeugen eher geringere Fortschritte. Die Wirkungsgrade von Elektromotoren, Steuerelektronik und Ladegeräten sind bereits heute auf einem hohen Niveau. Substanzielles Verbesserungspotenzial gibt es hingegen bei Nebenverbrauchern wie der elektrischen Heizung oder der Temperierung der Batterien. Gerade bei niedrigen Aussentemperaturen kann hier bis zur Hälfte des Energieverbrauchs verloren gehen.

Den grössten Entwicklungsschub erwarten wir aber bei den Batterien. Momentan sind die Kosten und die beschränkte Energiedichte der Batterien ein zentraler Hinderungsgrund für die Ausbreitung von Elektromobilität. Die Energiedichte der Batterien wird sich in den nächsten Jahrzehnten jedoch vervielfachen, wodurch sich sowohl die Batteriemasse im Fahrzeug reduzieren als auch eine höhere Reichweite erzielen lässt. Wir gehen davon aus, dass die mit einer 300-Kilogramm-Batterie in einem Kompaktklasseauto erreichbare Reichweite von heute etwa 150 Kilometer bis 2035 auf über 600 Kilometer ansteigt und somit in einem ähnlichen Bereich wie bei ICE-Fahrzeugen liegen wird. Auch erwarten wir, dass die Batterielebensdauer und -zuverlässigkeit soweit erhöht wird, dass innerhalb der betrachteten Lebensdauer eines Fahrzeugs kein Batterieersatz mehr nötig sein wird. 


\subsubsection{Umweltauswirkungen}

Die Ökobilanz ergibt für ICE-Fahrzeuge eine signifikante zukünftige Abnahme der $\mathrm{CO}_{2}$-Emissionen, während Elektrofahrzeuge auf tiefem Niveau ungefähr konstant bleiben oder - aufgrund des zwischenzeitlich «schmutzigeren» Stroms - ihre Umweltbelastung sogar steigen wird. Diese Stagnation der Elektrofahrzeuge im Vergleich zu den ICE-Vehikeln lässt sich mit dem noch brachliegenden Effizienzsteigerungspotenzial bei ICE-Fahrzeugen erklären, welches durch die strenge EU-Gesetzgebung (95g-CO - -Ziel für 2020) in den nächsten Jahren realisiert werden wird. Elektrofahrzeuge stossen dagegen in der Schweiz aufgrund der momentan sehr $\mathrm{CO}_{2}$-armen Stromproduktion beim Fahren kaum Treibhausgase aus, sind aber in der Fahrzeugherstellung, die in der Regel im Ausland erfolgt, deutlich umweltbelastender.

Dieser generelle Trend hin zu $\mathrm{CO}_{2}$-ärmerer Mobilität schlägt sich auch in den Ökobilanzergebnissen auf Szenarioebene nieder (Abbildung 48). Alle drei Szenarien weisen bereits 2020 eine deutliche $\mathrm{CO}_{2}$-Einsparung von 10 Prozent gegenüber 2012 auf, und selbst eine reine ICE-basierte Mobilität (grauer Balken) führt langfristig zu $\mathrm{CO}_{2}$-Einsparungen von 20 Prozent trotz einer gleichzeitig prognostizierten Zunahme der Mobilität um 24 Prozent. Nicht berücksichtigt sind dabei allfällige $\mathrm{CO}_{2}$-Einsparungen durch die Verwendung von Biotreibstoffen (siehe hierzu die TA-SWISS-Studie von Zah, Binder et al. 2010).

Überraschend ist, wie gering die Unterschiede zwischen den Szenarien ausfallen. Während 2020 die drei Szenarien praktisch identisch sind, beträgt das zusätzliche Sparpotenzial des optimistischen elektromobilitätsfreundlichen COMSzenarios gegenüber dem Business-as-Usual-Szenario im Jahr 203517 Prozent. Dieser Unterschied lässt sich einerseits mit den Unterschieden bei der Flottenzusammensetzung der Szenarien und andererseits mit dem auf 50 Prozent beschränkten $\mathrm{CO}_{2}$-Einsparungspotenzial der Elektrofahrzeuge erklären, wenn der ganze Lebenszyklus berücksichtigt wird.

Zusammenfassend lässt sich sagen, dass die Treibhausgasemissionen wegen Effizienzsteigerungen bei den ICE-Fahrzeugen und wegen der Verbreitung der Elektromobilität in den nächsten Jahren und Jahrzehnten signifikant zurückgehen werden. Die Elektromobilität ermöglicht eine zusätzliche Reduktion der Umweltbelastung durch geringere Lärmemission und Vermeidung von Auspuffemissionen wie Stickoxide und Partikel. Abgasemissionen sind bei modernen und zukünftigen ICE-Fahrzeugen zwar im Normbetrieb sehr tief, können aber bei 
einem Kaltstart um ein Vielfaches über dem Grenzwert liegen. Lärmemissionen werden bei geringen Geschwindigkeiten von Motorgeräuschen dominiert und fallen unter diesen Umständen für Elektrofahrzeuge deutlich geringer aus als für ICE-Fahrzeuge. Entsprechend ergeben sich in dieser Studie nicht quantifizierte Vorteile für elektrische Fahrzeuge in Städten, in denen oft kurze Distanzen bei relativ geringen Geschwindigkeiten gefahren werden. Elektromobilität bewirkt aber eine Zunahme von Treibhausgasemissionen und gesamthafter Umweltbelastung in den Vorketten der Produktion, welche sich auch nur beschränkt reduzieren lassen, beispielsweise durch Materialeinsparungen (Leichtbau) oder durch verstärkten «Re-Use» von Komponenten oder Recycling wertvoller Rohstoffe. Äusserst relevant für die Umweltbilanz der Elektromobilität ist ebenfalls die Art und Weise der Stromproduktion. Die Sensitivitätsanalysen der Studie zeigen auf, dass bereits bei der Verwendung des gegenwärtigen EU-Strommixes die Treibhausgaseinsparungen gegenüber den ICE-Fahrzeugen weitgehend zunichte gemacht werden. Von fundamentaler Bedeutung für die Umweltbilanz von Elektromobilität ist daher die Verfügbarkeit entsprechender Mengen von erneuerbarem Strom.

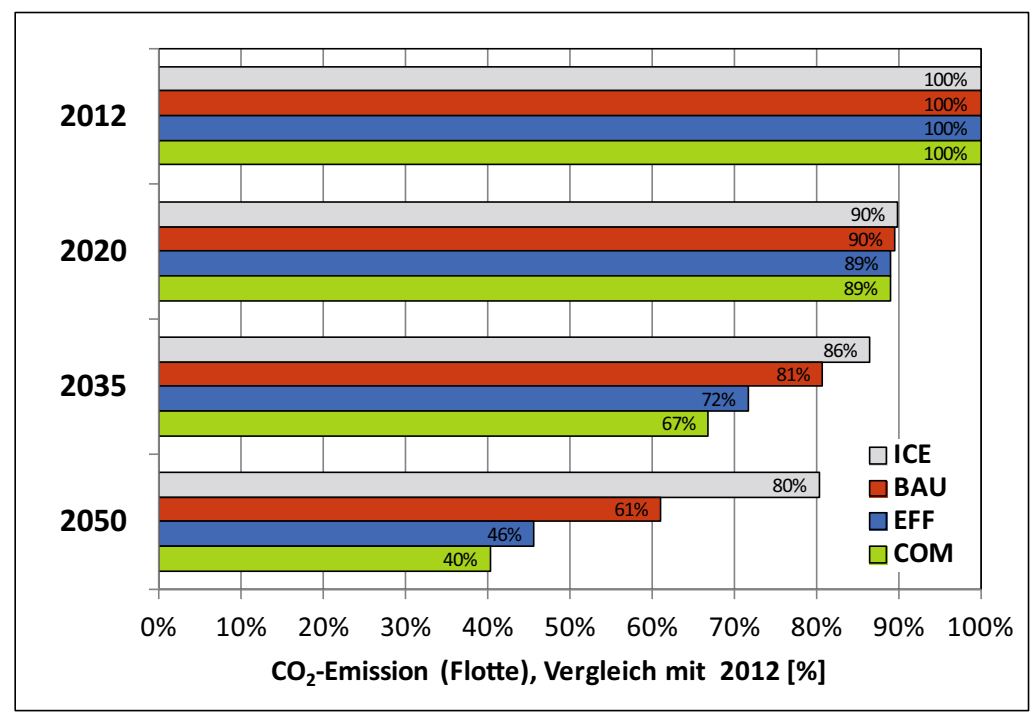

Abbildung 48: Gesamthafte Reduktion der Treibhausgasemissionen in den drei Szenarien BAU, EFF und COM und einer ausschliesslichen Optimierung der ICE-Fahrzeuge (ICE) gegenüber der Situation im Jahr 2012. 


\subsubsection{Verfügbarkeit des Stroms}

Abbildung 49 zeigt die Zusammensetzung des Schweizer Stromangebots gemäss Energiestrategie des Bundesrates. Die Abbildung zeigt auch, welche Anteile der prognostizierten Stromproduktion für die Mobilität auf Schiene und Strasse verfügbar sind - im Jahr 2050 max. 10 Prozent. Abbildung 50 ist eine Detailvergrösserung aus Abbildung 49, welche den für die Elektromobilität relevanten Teil detaillierter darstellt. Der Strombedarf für unsere drei Elektromobilitätsszenarien liegt demnach bis etwa 2035 leicht unter dem von den BFE-Energieperspektiven errechneten Bedarf.

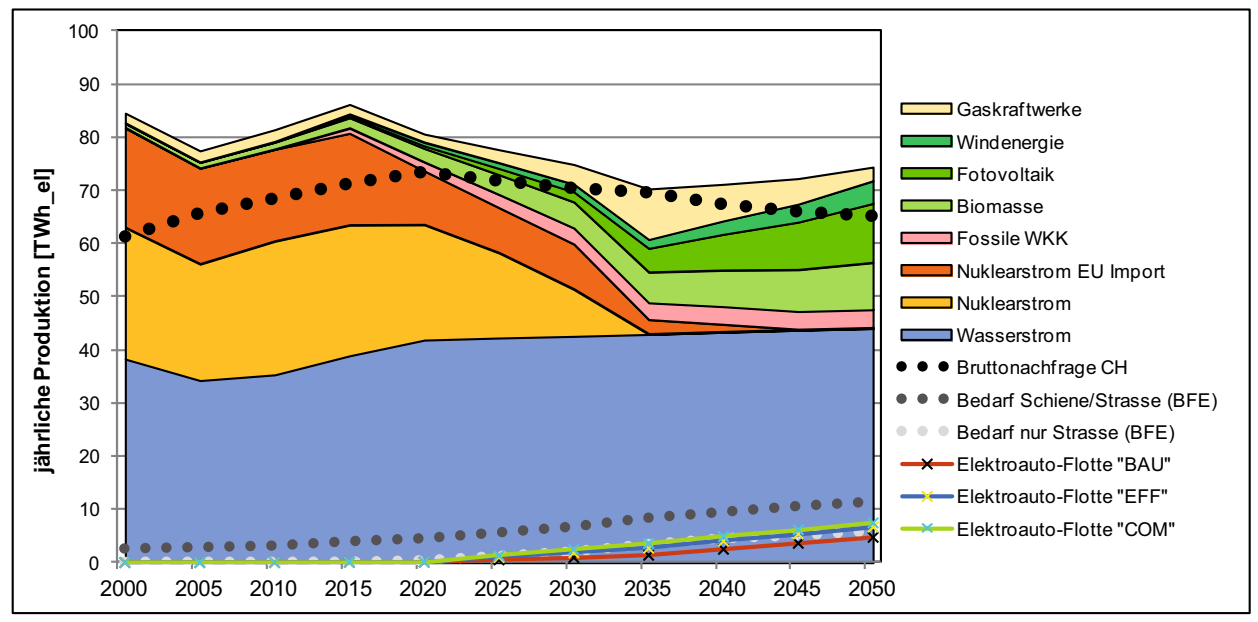

Abbildung 49: Verwendetes Stromangebotsszenario (Prognos 2012, farbige Flächen), gesamter und für die Mobilität alloziierter Stromverbrauch (gepunktete Linien), Strombedarf der drei Elektromobilitätsszenarien (ausgezogene Linien).

Interessanterweise ist gemäss Abbildung 49 der gesamte zukünftige Strombedarf der Elektromobilität ähnlich hoch wie die aus Erdgas produzierte Strommenge. Würde Erdgas direkt in Erdgas-Hybrid-Fahrzeugen genützt, so könnte eine ähnliche $\mathrm{CO}_{2}$-Reduktion wie bei Elektrofahrzeugen erreicht werden. Die Abhängigkeit der Mobilität von einer fossilen Energiequelle bliebe dabei allerdings erhalten. 


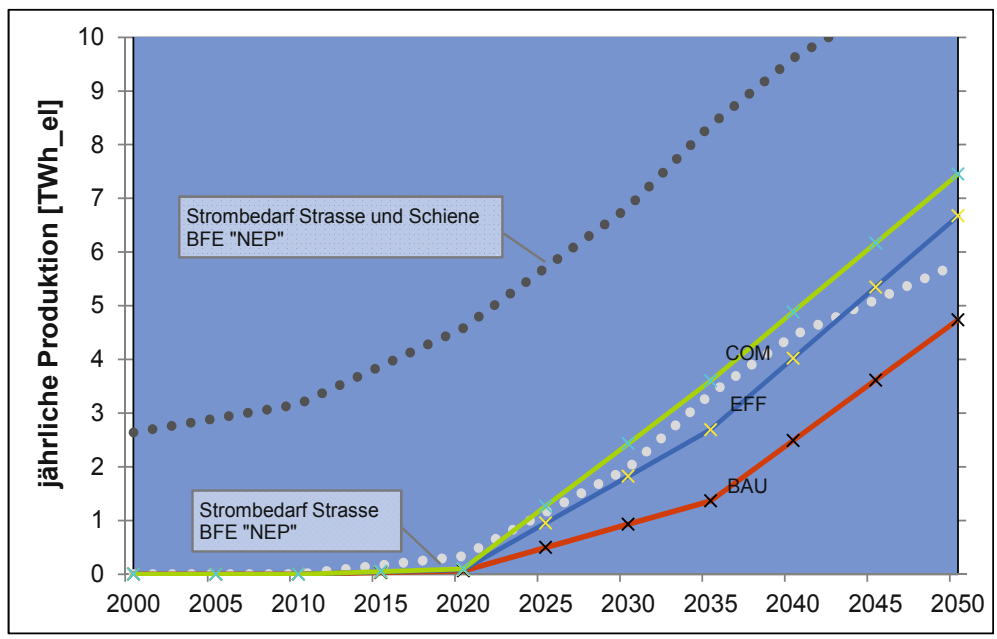

Abbildung 50: Detailvergrösserung aus Abbildung 49.

Erst nach 2040 steigt der Strombedarf unserer Szenarien EFF und COM über die zur Verfügung stehende Strommenge an. Mittelfristig sollten sich also alle drei Szenarien realisieren lassen, ohne dass zusätzlicher Strom zur Verfügung gestellt werden muss. Es gilt anzumerken, dass dies eine sehr grobe Netto-Betrachtung ist, welche die Schwankungen der Stromerzeugung und -nutzung während des Tages und im Verlauf der Jahreszeiten vernachlässigt.

\subsubsection{Rohstoffbeanspruchung}

Die Elektromobilität führt zu einer Zunahme der Anwendung von potenziell kritischen Rohstoffen wie beispielsweise Lithium oder Seltenerdelemente. Da diese Elemente auch in anderen Zukunftstechnologien wie Elektro- und Elektronikgeräten oder erneuerbaren Energien genutzt werden, kann die Rohstoffbeanspruchung der kritischen Rohstoffe in Zukunft stark steigen. Ob die Elektromobilität einer der grossen Treiber dieser Entwicklung sein wird, ist von verschiedenen Faktoren abhängig, wie beispielsweise der Reduktion der benötigten Mengen an Rohstoffen innerhalb der Anwendungsbereiche, der möglichen Substitution durch weniger kritische Rohstoffe oder der Nutzung von Recycling und Wiederverwendung der Rohstoffe. 
Mit zunehmender Primärproduktion wird der materielle und energetische Aufwand zur Gewinnung der Rohstoffe steigen und die Qualität der abgebauten Lagerstätten sinken. Für eine umweltschonendere Gewinnung dieser kritischen Rohstoffe ist es daher wichtig, diese an den Prinzipien der nachhaltigen Entwicklung zu orientieren und durch Rohstoffzertifizierungen und klare Vorgaben für Abbauunternehmen zu unterstützen.

Betreffend der drei Szenarien kann davon ausgegangen werden, dass eine höhere und raschere Penetration der Elektromobilität grundsätzlich zu kurzfristigen Versorgungslücken für bestimmte Elemente führen könnte. Da dies jedoch stark von der weltweiten Nachfrage der Elektromobilität sowie anderen Technologien abhängig ist und weniger von der Entwicklung in der Schweiz, ist eine Aussage aufgrund der drei Szenarien schwierig.

\subsubsection{Volkswirtschaftliche Beurteilung}

Tabelle 26 zeigt die verschiedenen involvierten Akteure und Aktivitäten, wie in Kapitel 7.2 beschrieben. Für jedes Aktivitätsfeld wird die Entwicklung gemäss der Skala in Tabelle 27 beurteilt.

\begin{tabular}{lccccc}
\hline Aktivitäten & Forschung & Rohstoffabbau & Produktion & Nutzung & $\begin{array}{c}\text { Entsorgung / } \\
\text { Recycling }\end{array}$ \\
\hline Wirtschaft & $\checkmark$ & $\approx$ & $(\checkmark)$ & $(\checkmark)$ & $\checkmark$ \\
Gesellschaft & $(\checkmark)$ & $\approx$ & $(\checkmark)$ & $\checkmark$ & $(\checkmark)$ \\
Bund & $(\checkmark)$ & $\approx$ & $(\checkmark)$ & $+/-$ & $(\checkmark)$ \\
\hline
\end{tabular}

Tabelle 26: $\quad$ Akteure und Aktivitäten der Elektromobilität.

\begin{tabular}{|c|l|}
\hline$\checkmark$ & Sehr positive Entwicklung \\
\hline$(\checkmark)$ & Positive Entwicklung \\
\hline$\approx$ & Limitierter Einfluss \\
\hline$+/-$ & Entwicklung noch unklar \\
\hline
\end{tabular}

Tabelle 27: Beurteilungsskala. 
Tabelle 26 verdeutlicht, dass die Entwicklung der Elektromobilität für die Schweizer Wirtschaft und Gesellschaft positiv ausfällt. Die Elektromobilität bietet zusätzliche Forschungs- und Innovationsmöglichkeiten, die Wertschöpfung, neue Arbeitsplätze und Steuereinnahmen in der Schweiz generieren. Auf die Rohstoffbereitstellung wird die Schweiz auch in Zukunft nur einen beschränkten Einfluss haben, da die für die Elektromobilität benötigten Rohstoffe im Ausland abgebaut werden und darauf nur durch internationale Vorstösse und durch die Überwachung der Einhaltung bestehender OECD-Richtlinien Einfluss genommen werden kann. Aufgrund der in Kapitel 6 diskutierten Aspekte ist es sehr wichtig, sicherzustellen, dass der Abbau der nötigen Rohstoffe nachhaltig gestaltet und die Nutzung von Sekundärrohstoffen maximiert wird.

Schweizer Firmen sind bereits heute in der Bereitstellung von spezifischen Produkten für die Elektromobilität tätig. Mit steigender Nachfrage nach Elektromobilitätskomponenten kann dieser Markt in der Schweiz gestärkt und sogar ausgebaut werden. Dies führt zu mehr Arbeitsplätzen und einer höheren Standortattraktivität der Schweiz. In der Nutzungsphase hat die Elektromobilität vor allem für die Gesellschaft positive Auswirkungen, hauptsächlich durch gesundheitliche Verbesserungen aufgrund tieferer Lärm- und Schadstoffemissionen. Für die Wirtschaft haben die inländische Energiebereitstellung und die dadurch reduzierte Abhängigkeit von importierten, fossilen Rohstoffen einen positiven Effekt. Nur bei den Bundeseinnahmen ist die Situation nicht ganz klar, da diese stark von der zukünftigen Entwicklung der Mineralölsteuer und einer möglichen kilometerabhängigen Besteuerung abhängt. Auch im Bereich des Recyclings hat die Schweiz ein grosses Know-how, das zu verstärkter Innovation in diesem Bereich und einer höheren Wertschöpfung führen kann.

Für die drei verschiedenen Szenarien gilt, dass eine frühe und stärkere Penetration der Elektromobilität, wie es das COM-Szenario und teilweise das EFF-Szenario beschreiben, für die Schweiz positive volkswirtschaftliche Einflüsse haben kann (namentlich im Hinblick auf Gesamtenergieeffizienz des MIV und Integration neuer erneuerbarer Energien im Energiesystem). Es muss jedoch darauf geachtet werden, dass die Schweizer Wirtschaft imstande ist, einer schnellen Entwicklung zu folgen und den Anschluss an andere innovative Wirtschaftsregionen nicht verliert. Daher ist es wichtig, Innovation und Forschung im Bereich der Elektromobilität zu ermöglichen und zu fördern sowie die Marktbedingungen und die Standortattraktivität der Schweiz für die Herstellung von Komponenten der Elektromobilität zu optimieren. 


\subsection{Gesamtbeurteilung und Fazit}

Die folgende Grafik zeigt die Übersicht der im Kapitel 7 evaluierten Teilziele. Zusätzlich zu den evaluierten Mobilitätstypen Elektromobilität (EV) und treibstoffbasierte Mobilität (ICE) wird die Entwicklung in den drei Szenarien qualitativ evaluiert.

Die Umweltindikatoren machen deutlich, dass für beide Mobilitätstypen die Bedingungen entweder unverändert bleiben oder sich verbessern. Mit der Elektromobilität verbessern sich die Umweltbedingungen jedoch stärker als mit der treibstoffbedingten Mobilität. Für die drei Szenarien zeigt das BAU-Szenario eine weniger starke Verbesserung der Umweltbedingungen als die beiden Szenarien EFF und COM. Ungewiss ist die Entwicklung bei beiden Mobilitätstypen betreffend dem Einsatz von metallischen Rohstoffen und der Erhaltung der natürlichen Lebensräume. Der höhere ÖV-Modalsplit im COM-Szenario erlaubt aber den bestmöglichen Erhalt dieser Lebensräume.

Auch bei den Wirtschaftsindikatoren weisen die Elektromobilität und die beiden Szenarien EFF und COM positive Entwicklungen auf. Dies vor allem aufgrund der möglichen Innovation und der wirtschaftlichen Möglichkeiten, welche die neue Technologie mit sich bringt. Grosse Herausforderungen sind dabei jedoch die Kosten für die Fahrzeuge und die nötige Infrastruktur sowie die Verfügbarkeit der Rohstoffe.

Die Gesellschaftsindikatoren zeigen, dass die Elektromobilität sowie die Szenarien EFF und COM positive Auswirkungen auf die Verkehrssicherheit haben können. Es ist jedoch unklar, wie sich die Elektromobilität auf die Störfallrisiken und die Veränderung des Siedlungs- und Landschaftsbildes auswirkt. Allerdings wird die stärkere Berücksichtigung des ÖV für längere Fahrten im COM-Szenario das Landschaftsbild weniger stark beeinträchtigen.

Insgesamt hat die Elektromobilität, mit dem heutigen Strommix in der Schweiz und der angenommenen Weiterentwicklung des Strommixes, mehrheitlich positive Auswirkungen auf Volkswirtschaft und Umwelt. Die grossen Herausforderungen bestehen bei den Kosten für die Fahrzeuge sowie bei der Infrastruktur und der Verknappung der Rohstoffe. Für die einzelnen Szenarien sehen die Auswirkungen tendenziell positiv aus, da diese auf einem Mix aus Elektromobilität und treibstoffbasierter Mobilität beruhen. Je früher die Elektromobilität eingesetzt wird und je höher ihr Anteil ist, desto positiver sind die Auswirkungen. Dies ist vor allem bei den Wirtschaftsindikatoren wichtig, da sich die wirtschaftliche Entwick- 
lung bei einer stärkeren Penetration der Elektromobilität verstärkt. Unter Verwendung eines anderen Strommixes würde sich ein teilweise anderes Bild ergeben. Im nächsten Kapitel erörtern wir, ob sich technologiespezifische Anreize oder auf Energieeffizienz fokussierte energiepolitische Instrumente besser eignen.

\begin{tabular}{|c|c|c|c|c|c|c|}
\hline & & ICE & EV & BAU & EFF & COM \\
\hline \multicolumn{7}{|c|}{ Umwelt } \\
\hline U1.1 & Luftschadstoffe senken & $(\checkmark)$ & $\checkmark$ & $(\checkmark)$ & $\checkmark$ & $\checkmark$ \\
\hline U1.2 & Lärmbelastung senken & $(\checkmark)$ & $\checkmark$ & $(\checkmark)$ & $\checkmark$ & $\checkmark$ \\
\hline U2.1 & Treibhausgasemissionen senken & $(\checkmark)$ & $\checkmark$ & $(\checkmark)$ & $\checkmark$ & $\checkmark$ \\
\hline U3.1 & Primärenergieverbrauch senken & $(\checkmark)$ & $\checkmark$ & $(\checkmark)$ & $\checkmark$ & $\checkmark$ \\
\hline U3.2 & Bodenversiegelung reduzieren & $\approx$ & $\approx$ & $\approx$ & $\approx$ & $\approx$ \\
\hline U3.3 & Einsatz metallischer Rohstoffe minimieren & $+/-$ & $+/-$ & $+/-$ & $+/-$ & $+/-$ \\
\hline U4.1 & Natürliche Lebensräume erhalten & $+/-$ & $+/-$ & $+1-$ & $+/-$ & $(\checkmark)$ \\
\hline U4.2 & Landschaftszerschneidung reduzieren & $\approx$ & $\approx$ & $\approx$ & $\approx$ & $\approx$ \\
\hline \multicolumn{7}{|c|}{ Wirtschaft } \\
\hline W1.1 & $\begin{array}{l}\text { Investitions- und Betriebskosten für } \\
\text { Fahrzeuge minimieren }\end{array}$ & ! & ! & ! & ! & ! \\
\hline W1.2 & $\begin{array}{l}\text { Investitions- und Betriebskosten für } \\
\text { Infrastruktur minimieren }\end{array}$ & $\approx$ & ! & ! & ! & ! \\
\hline W2.1 & $\begin{array}{l}\text { Absatzmärkte für Schweizer } \\
\text { Unternehmen schaffen }\end{array}$ & $\approx$ & $(\checkmark)$ & $\approx$ & $(\checkmark)$ & $(\checkmark)$ \\
\hline W2.2 & $\begin{array}{l}\text { Einheimische Rohstoffe und Produkte } \\
\text { nutzen }\end{array}$ & $\approx$ & $(\checkmark)$ & $\approx$ & $(\checkmark)$ & $(\checkmark)$ \\
\hline W3.1 & $\begin{array}{l}\text { Unterstützung einer regional } \\
\text { ausgeglichenen wirtschaftlichen } \\
\text { Entwicklung }\end{array}$ & $\approx$ & $(\checkmark)$ & $\approx$ & $(\checkmark)$ & $(\checkmark)$ \\
\hline W3.3 & $\begin{array}{l}\text { Flexibilität des Systems erhöhen } \\
\text { (Investitionsrisiko, Anpassungsfähigkeit) }\end{array}$ & $\approx$ & $(\checkmark)$ & $\approx$ & $(\checkmark)$ & $(\checkmark)$ \\
\hline W4.1 & $\begin{array}{l}\text { Abhängigkeit von einzelnen } \\
\text { Energieträgern senken }\end{array}$ & $\approx$ & $\checkmark$ & $\approx$ & $\checkmark$ & $\checkmark$ \\
\hline W4.2 & $\begin{array}{l}\text { Verfügbarkeit der Rohstoffe } \\
\text { gewährleisten }\end{array}$ & ! & ! & ! & ! & ! \\
\hline \multicolumn{7}{|c|}{ Gesellschaft } \\
\hline G1.1 & Verkehrssicherheit erhöhen & $\approx$ & $\checkmark$ & $\approx$ & $\checkmark$ & $\checkmark$ \\
\hline G1.2 & Minimierung von Störfallrisiken & $\approx$ & $+1-$ & $+/-$ & $+/-$ & $+/-$ \\
\hline G1.3 & $\begin{array}{l}\text { Beeinträchtigungen des Siedlungs- und } \\
\text { Landschaftsbildes minimieren }\end{array}$ & $+/-$ & $+/-$ & $+1-$ & $+1-$ & $(\checkmark)$ \\
\hline G2.1 & Zugang für alle Regionen und & $\approx$ & $\approx$ & $\approx$ & $\approx$ & $\approx$ \\
\hline
\end{tabular}




\subsection{Diskussion und Empfehlungen zu den Leitfragen}

Im Folgenden gliedern wir die Diskussion der wichtigsten Aspekte und Erkenntnisse aus der vorliegenden Studie nach den sechs am Schluss von Kapitel 1.1 formulierten Fragen.

Wie könnten sich die unterschiedlichen Formen von Elektrofahrzeugen weiterentwickeln? Welche Marktpenetrationsverläufe und Marktanteile sind minimal und maximal möglich?

In den nächsten Jahren wird die Verbreitung der Elektromobilität nur sehr langsam stattfinden - wir prognostizieren für 2020 Verkaufsanteile, die deutlich unter 5 Prozent liegen. Ursachen hierfür sind die gegenwärtig hohen Verkaufspreise und die noch geringen Produktionskapazitäten für Elektrofahrzeuge, aber auch die kurzen Reichweiten, die den Einsatz der Fahrzeuge vorläufig nur in Nischenmärkten erlauben. Nach 2020 werden die Verkaufszahlen jedoch aufgrund von Fortschritten bei der Batterietechnologie mit entsprechend grösseren Fahrzeugreichweiten und Kostenreduktionen rasch ansteigen und 2035 Verkaufsanteile von 25 bis 55 Prozent erreichen. Die Penetration der Elektromobilität erfolgt primär über kleine Fahrzeugtypen, da dort ihr grösster Nutzen liegt (geringes Batteriegewicht und hohes Einsparpotenzial bei urbanem Einsatz). Die Penetration wird durch die Technologieentwicklung (im COM-Szenario zusätzlich durch eine Veränderung des Mobilitätsverhaltens) bestimmt und nicht durch Fördermassnahmen erzwungen. Deshalb sind die Unterschiede zwischen den Szenarien bis 2035 gering, zeigen sich dann aber bis 2050 immer deutlicher. Weil es etwa 15 Jahre dauert, bis sich die Trends am Neuwagenmarkt im Fahrzeugpark manifestieren, dauert es bis zirka 2070, bis die Unterschiede zwischen den Szenarien voll zum Tragen kommen.

Chancen: Die Chancen einer zukünftigen Verbreitung der Elektromobilität liegen bei der Diversifizierung des Energieverbrauchs der Mobilität. Strom kann auf sehr unterschiedliche Arten bereitgestellt werden, unter anderem auch aus verschiedenen erneuerbaren Energiequellen. Dadurch kann die einseitige Abhängigkeit von fossilen Energiequellen vermindert werden. Das ist vor allem bei einer langfristigen Betrachtung über 2050 hinaus von Bedeutung. Elektromobilität 
führt aber auch zu einer Diversifizierung der Fahrzeugtypen. Bereits heute ist eine neuartige Klasse von elektrischen Kleinstwagen in Leichtbauweise am Markt erhältlich (z.B. Renault Twizzy), die aufgrund ihrer leichten und wendigen Bauweise optimal für den Einsatz im städtischen Verkehr ist. Durch den Einsatz von jeweils optimalen Fahrzeugtypen anstelle von «Alleskönnern» könnte signifikant Energie gespart werden.

- Effizienzorientierte Politik bei Neuwagen statt gezielter Elektromobilitätsförderung: Unabhängig von der Antriebstechnologie kann durch Effizienzsteigerung eine Verbesserung praktisch aller Nachhaltigkeitsindikatoren erreicht werden. Wie bereits besprochen, sind die Umstellung der energiepolitischen Zielgrössen auf die Gesamtenergie (ausgedrückt in Primärenergie-Benzinäquivalenten) und die politische Zielgrösse der Gesamtenergieeffizienz neuer Personenwagen indirekt ein Mittel zur Förderung der Elektromobilität. Je nach Verlauf der technologischen Innovation werden sich dabei hocheffiziente ICE oder EV schneller Marktanteile sichern; dies erlaubt es, den Markt spielen zu lassen und minimiert das Risiko falscher Anreize infolge von technologiespezifischen Massnahmen.

- Synergien mit Verkehrstelematik: Technische Massnahmen wie automatische Abstandsmessung und elektronische Steuerung der Fahrzeuge erhöhen die Sicherheit. Diese Entwicklung ist unabhängig von der Entwicklung der Elektromobilität. Sie fördert aber die Sicherheit und damit indirekt die Elektromobilität, weil der Elektroantrieb nicht nur heute, sondern auch in Zukunft vor allem bei Kleinwagen seine Stärken ausspielen kann. Mehr aktive Sicherheit begünstigt Kleinwagen - sie begünstigt damit indirekt kleinere und sparsamere Autos und darum implizit auch die Elektromobilität.

Risiken: Die Elektromobilität führt zu einer Verlagerung der Schadstoffemissionen aus dem Verkehr in die Vorketten der Energie- und Fahrzeugproduktion und damit auch aus dem Inland ins Ausland, was wiederum relevant für die schweizerische Klimapolitik ist. Auch bei den immer effizienteren ICE-Fahrzeugen findet diese Verlagerung statt, wenn auch weniger ausgeprägt. Der Grossteil der Umweltbelastung kann dadurch nicht mehr direkt gemessen werden, sondern muss basierend auf Annahmen zu den Produktionsbedingungen von Experten modelliert werden. Während sich der Nutzer am vermeintlich emissionsfreien Fahren freut, bleibt damit das Bewusstsein für die tatsächliche Umweltbelastung auf der Strecke. 
- Die Angaben auf der Energieetikette sollten den Well-to-Wheel-Vergleich zwischen Elektro- und ICE-Fahrzeugen aufgrund der benötigten Gesamtenergie erlauben. Mittelfristig ist der Wechsel vom Normverbrauch zum Realverbrauch anzustreben.

- Längerfristig braucht der Staat Informationen und Abschätzungen zur gesamten Umweltbelastung eines Fahrzeugs, inklusive Herstellung und Entsorgung. Dazu braucht es bereits kurzfristig ein entsprechendes Konzept. Noch offen ist, inwieweit auch die Kundeninformation auf die gesamte Umweltbelastung ausgelegt werden kann und soll oder ob hier als approximative Zielgrösse die Gesamtenergie verständlicher ist.

Wie viel Strom wird zusätzlich benötigt? Wie können Elektrofahrzeuge den grösstmöglichen positiven Nutzen erbringen?

Aufgrund der hohen Effizienz des Antriebsstrangs brauchen Elektrofahrzeuge relativ wenig Strom. Mit 9 Prozent der Schweizer Stromerzeugung liessen sich beispielsweise im Jahr 205065 Prozent der motorisierten Individualmobilität betreiben. Gegenwärtig ist der Stromverbrauch von Nebenverbrauchern wie der Batterie-Konditionierung oder der Heizung noch sehr bedeutend und kann bei niedrigen Aussentemperaturen fast gleich hoch sein wie der Stromverbrauch des Antriebs. Hier könnte in den nächsten Jahren ein beträchtliches Sparpotenzial realisiert werden.

Der zusätzliche Strombedarf der Elektromobilität muss auch im Kontext der zeitlich hochdynamischen Stromproduktion und -nutzung betrachtet werden. Da der Ladezeitpunkt bei Elektrofahrzeugen oftmals zeitlich flexibel ist (Standzeit über Nacht, mehrtägiges Nichtbenutzen des Fahrzeugs), können die Ladevorgänge an die kurzfristige Auslastung des Stromnetzes angepasst werden (Smart Charging) und so die Netzauslastung optimieren.

Chancen: Im Gegensatz zu chemischen Treibstoffen, die entweder aus fossilen Ressourcen oder aus Biomasse hergestellt werden, kann Strom aus einer Vielzahl unterschiedlicher Primärenergiequellen produziert werden. Dies eröffnet neue Optionen zur Substitution fossiler Energiequellen und zur Reduktion von Treibhausgasemissionen. Allerdings ist es beim Stromtanken - im Gegensatz zum Tanken fossiler Treibstoffe - sehr schwierig, die konkrete Stromquelle zu identifizieren. 
- Durch die Einführung spezifischer Abrechnungsmodelle für den Strombezug, wie beispielsweise einer Solarstromvignette, kann die Verantwortung für die Vorkette durch den Endkunden wahrgenommen werden.

- Die Einführung dynamischer Strompreise (mit Viertelstundenauflösung) macht die zeitliche Flexibilisierung der Ladevorgänge (Smart Charging) ökonomisch attraktiv.

Risiken: Während die fossil betriebene Mobilität auf einer unabhängigen Energieinfrastruktur aufbaut, stützt sich die Elektromobilität auf das bereits heute intensiv genutzte Stromnetz ab. Auch kann der unkontrollierte Strombezug der Elektromobilität zum ungewollten «Anzapfen» fossiler Stromquellen und damit zu einer substanziellen Erhöhung der Treibhausgasemissionen führen.

- Der Ausbau der Elektromobilität sollte parallel mit einem Ausbau des Stromnetzes erfolgen, welcher die zusätzlichen Anforderungen der Elektromobilität berücksichtigt und die Rückverfolgbarkeit der Stromquellen erlaubt (Smart Grid).

- Die Zahlungsbereitschaft der Konsumenten für Mobilität ist hoch. Möglicherweise reicht die (aus gesamtwirtschaftlicher Sicht maximal mögliche) Dynamisierung der Strompreise nicht aus, um Kunden aktiv zu Smart Charge zu bewegen. Es sind deshalb auch Vorschriften zu prüfen und Geschäftsmodelle (Batterieleasing inkl. Strommix und Strommanagement) zu ermöglichen, die dazu führen, dass die Flexibilisierung der Ladevorgänge zum Standard wird und vom Kunden im Einzelfall aktiv «overruled» werden muss.

Wie wichtig ist die Rolle seltener Materialien für Batterien und Permanentmagnete? Könnte es zu Knappheit für andere Technologiezweige kommen?

Die Elektromobilität wird zu einer Zunahme der Nachfrage nach kritischen Rohstoffen führen. Besonders deutlich wird die Zunahme bei Lithium ausfallen, dessen globale Nachfrage wesentlich durch die Elektromobilität sowie andere Elektro- und Elektronikgeräte bestimmt sein wird. Aber auch die Seltenerdelemente könnten im Bereich der Elektromobilität vermehrt zur Anwendung kommen, beispielsweise in leistungsfähigen Permanentmagneten. 
Hauptursache für die potenzielle Knappheit einzelner mineralischer Rohstoffe ist weniger deren geologische Verfügbarkeit als vielmehr die Konzentration des Angebots auf wenige Länder oder Firmen, verbunden mit fehlenden Substitutionsmöglichkeiten und geringen Recyclingraten.

Chancen: Viele der potenziell knappen Metalle lassen sich im Mobilitätssektor substituieren. So lassen sich beispielsweise auch Elektromotoren ohne neodymhaltige Permanentmagnete bauen und es gibt Batteriekonzepte, die (ausser Lithium) keine weiteren kritischen Metalle enthalten.

Risiken: Der zukünftige Bedarf an gewissen kritischen Metallen (z.B. Neodym oder Lithium) wird um Faktoren ansteigen, wenn Elektrofahrzeuge im grossen Stil auf den Markt kommen. Viele dieser kritischen Metalle werden aber auch in anderen Wachstumssektoren (z.B. erneuerbare Energie oder ICT) benötigt und stehen so in Nutzungskonkurrenz.

- Durch verstärktes Recycling, den effizienteren Einsatz oder die Substitution der Rohstoffe lässt sich die Abhängigkeit von kritischen Metallen reduzieren.

Gibt es spezifischen Handlungsbedarf für die nachhaltige Entsorgung von Elektrofahrzeugen und das Schliessen der Stoffkreisläufe?

Die Ergebnisse der Ökobilanzierung zeigen, dass der Ressourcenverbrauch in der Elektromobilität zu mehr als 80 Prozent bei der Produktion des Elektrofahrzeugs liegt, während der Ressourcenverbrauch bei Fahrzeugen mit Verbrennungsmotor heute zu 75 Prozent in der Betriebsphase anfällt, wobei auch hier die Tendenz sinkend ist. Zusätzlich verschiebt sich die Materialzusammensetzung bei Elektrofahrzeugen hin zu einer komplexeren Mischung seltener und wertvoller Metalle.

Während heute der Umweltfokus immer noch auf der Nutzungsphase der Mobilität liegt (Reduktion der Auspuffemissionen, Erhöhung der Fahrzeugeffizienz), sollte sich in der Zukunft das Interesse auf Verbesserungen bei Fahrzeugproduktion und Recycling konzentrieren. 
Chancen: Die Rückgewinnung der Rohstoffe ist angesichts der global steigenden Nachfrage und der negativen Umweltwirkungen beim Abbau wichtig. Das Augenmerk ist insbesondere auf die Entwicklung bei bisher weniger gebräuchlichen Rohstoffen zu legen, die mit der vermehrt eingesetzten Elektronik sowie der steigenden Anzahl Batterien wichtiger werden. Handlungsbedarf aus staatlicher Sicht:

- Vorschriften, die die einfachere Trennung und Wiederbenutzung bestimmter Komponenten und Materialien durch entsprechendes Fahrzeugdesign erleichtern (Design for Recycling).

- Vorschriften, welche Komponenten vor dem Schreddern zwingend aus dem Fahrzeug zu entfernen und separaten Verwertungskanälen zuzuführen sind; hier kann neben den Batterien auch die Elektronik erfasst werden.

- Vorschriften, wie geschredderte Fahrzeuge (Resh) weiter zu verarbeiten und stofflich aufzutrennen sind.

Risiken: Der tendenziell steigende Ressourcenbedarf bei der Fahrzeugproduktion, insbesondere bei kritischen Metallen, führt zu einer Verknappung und Verteuerung einzelner Metalle. Da die meisten kritischen Metalle auch in anderen wachsenden Sektoren (erneuerbare Energien, ICT) eingesetzt werden, wirkt sich der Boom bei der Elektromobilität negativ auf andere Trends aus.

- Substitution knapper Rohstoffe; Erhöhung der Rückgewinnungsrate knapper Rohstoffe aus Altfahrzeugen; Rohstoffrecycling als globales Geschäft und dadurch Einbezug von Altautos in Enwicklungsländern.

Sind Reboundeffekte zu erwarten, lassen sich diese vermeiden? Wann müssten Einnahmenausfälle der öffentlichen Hand durch neue Steuersubstrate kompensiert werden?

Die heute hauptsächlich über die Erhebung von Mineralölsteuern erfolgende Finanzierung der Strasseninfrastruktur steht zur Diskussion. Auch ohne einen namhaften Anteil an Elektromobilen werden die Steuererträge zurückgehen, weil die Personenwagen energieeffizienter werden. Die Elektromobilität verstärkt und beschleunigt diese Entwicklung, weil es technisch kaum umsetzbar ist, analog 
zu fossilen Treibstoffen bei Elektrofahrzeugen die benötigte Strommenge separat zu erfassen und zu besteuern.

Chancen: Elektromobilität kann zu einer schnelleren Akzeptanz der Einführung einer kilometerabhängigen Besteuerung führen und eignet sich auch zur Illustration der Vorzüge einer tariflichen Differenzierung nach Energieeffizienz.

- Aus Sicht der Studienverfasser besteht kein Anlass, den Wechsel hin zu einer kilometerabhängigen Besteuerung zu verzögern, um damit die Elektromobilität zu fördern. Idealerweise wird die kilometerabhängige Besteuerung nach der Primärenergieeffizienz abgestuft.

Risiken: Die steuerliche Ungleichbehandlung von Benzin und Elektrizität führt zu niedrigen marginalen Energiekosten und kann damit trotz höherer Anschaffungskosten von Elektrofahrzeugen zu Mehrverkehr führen (finanzieller Reboundeffekt). Die wahrgenommene geringe Umweltbelastung durch Elektromobile kann zur Substitution von ÖV und Langsamverkehr durch Elektromobile führen (mentaler Reboundeffekt) - für den Vergleich des Energieaufwands von MIV mit ÖV siehe Spielmann und de Haan (2008). Wird die Energieeffizienz neuer PKW über deren $\mathrm{g} \mathrm{CO}_{2} / \mathrm{km}$-Wert energiepolitisch "gesteuert» und gleichzeitig für Elektrofahrzeuge ein weitgehend $\mathrm{CO}_{2}$-freier Strommix unterlegt, führt jede Zunahme des Anteils der Elektromobile zur Aufweichung des Effizienzziels für Fahrzeuge mit fossilen Brennstoffen (regulatorischer Rebound).

- Die Besteuerung auf Basis der zurückgelegten Kilometer, unabhängig vom Treibstoff, dämmt den finanziellen Reboundeffekt ein. Zu bevorzugen ist eine Besteuerung, welche auch von der Energieeffizienz des Fahrzeugs abhängt. Auf diese Weise führt auch eine baldige Einführung einer solchen Besteuerung für Elektromobile zu mehr Vor- als Nachteilen.

- Die Konsumenteninformation sollte vermehrt auf Primärenergie-Äquivalente fokussieren, um die bei einer Vermischung von Energie- und Klimapolitik entstehenden mentalen Reboundeffekte zu mindern.

- Bei der Übernahme von $\mathrm{g} \mathrm{CO}_{2} / \mathrm{km}$-Effizienzzielen der EU in der Schweiz müsste auch der anzunehmende EU-Strommix übernommen werden; andernfalls müssten treibstoffspezifische Unterziele festgelegt werden. 
Sollen allgemein energieeffiziente Fahrzeuge gefördert werden oder spezifisch Elektrofahrzeuge?

Die positiven Aspekte einer schnelleren Penetration der Elektromobilität bedeuten nicht, dass technologiespezifische finanzielle Anreize eingesetzt werden müssen. Zur Hauptsache führen technologieneutrale Massnahmen wie Mindestvorschriften, Maximalwerte sowie absatzgewichtete Mittelwerte zur Gesamtenergieeffizienz von Personenwagen indirekt zu einer Förderung der Elektromobilität. Je nach Verlauf der technologischen Entwicklung bei den Verbrennungsmotoren einerseits und der Batterietechnologie anderseits hat diese Strategie auch den Vorteil, dass sie robust ist im Hinblick auf nicht genau planbare technologische Innovationen.

Chancen: Die Reichweitenbeschränkung von Elektromobilen kann als Chance gesehen werden, weil sie vor allem in Kombination mit der Verwendung des ÖV für längere Distanzen geeignet sind.

- Gerade bei kleineren Fahrzeugen mit reduzierten Leistungsreserven sind Elektrofahrzeuge im Vorteil. Auf Energieeffizienz ausgerichtete Politikinstrumente führen deshalb zu einer für die Elektromobilität positiven indirekten Rückkopplung.

- Die offensive Nutzung neuer IT-Technologien für die bessere Verknüpfung des MIV für die erste und letzte Meile mit ÖV-Haltestellen und -Knoten fördert erstens indirekt die Elektromobilität (weil damit die Reichweitenproblematik an Relevanz verliert), zweitens generell den Einsatz von kleineren, leichteren (vor allem für kürzere Strecken geeigneten) Fahrzeugen.

- Es kann vermutet werden, dass das Vorhandensein von Elektromobilen die politische Akzeptanz für Mindestvorschriften für die Gesamtenergieeffizienz neuer Personenwagen erhöht.

Risiken: Solange Elektrofahrzeuge noch deutlich teurer sind als vergleichbare Fahrzeuge mit konventionellem Verbrennungsmotor, kann über technologiespezifische Subventionen versucht werden, schneller höhere Absatzzahlen zu erreichen und damit Skaleneffekte zu ermöglichen. Entsprechende Steuerrabatte oder Subventionen gibt es bereits in mehreren europäischen Ländern. In der 
Schweiz kennen die meisten Kantone die teilweise oder vollständige Befreiung von der Motorfahrzeugsteuer für Elektrofahrzeuge - rein finanziell geht es hier im Vergleich zum Ausland jedoch um geringere Anreize. Wenn der Markt aber noch angebotslimitiert ist, sind technologiespezifische Subventionen nicht effektiv. Wenn auch technisch schlechte Elektrofahrzeuge von einer Förderung profitieren, kann dies die politische Akzeptanz solcher Subventionen reduzieren.

- Kantonale Motorfahrzeug-Steuerrabatte künftig nur noch für Elektromobile mit hoher Energieeffizienz (Kategorie A der Energieetikette);

- Technologiespezifische Subventionen nur einführen, wenn der Markt nicht angebots-, sondern nachfragelimitiert ist;

- Befristung technologiespezifischer Subventionen und Steuerrabatte; die Befristung kann zeitlich, über den prozentualen Marktanteil oder über die Gesamtzahl der verkauften Einheiten erfolgen (analog zum Ansatz der USA für Hybridfahrzeuge).

\subsection{Mögliche Massnahmen aus der Perspektive der Stakeholder}

Im Folgenden werden, in fünf Gruppen gegliedert, die für eine nachhaltigere Entwicklung der individuellen Mobilität möglichen Massnahmen vorgestellt:

- Hardware: Infrastruktur, Treibstoff, Tankstellen, Normen. Dies betrifft die Strassen- und Versorgungsnetze sowie die Verteilung der Treibstoffe (Tankstellen, Ladestationen etc.)

- Angebotsseite (Fahrzeuge): Sicherstellung des Angebots benötigter Fahrzeuge

- Nachfrageseite: Akzeptanz und Marktpenetration

- Monetäre Aspekte: Finanzierung, Anreize, Subventionen und Steuern. Diese beziehen sich vor allem auf die Kosten des Fahrzeugs und der Infrastruktur

- Forschung 
Bei jedem Punkt werden vereinfacht die folgenden Akteure unterschieden: Bund, Kantone, Gemeinden, Autobranche (Hersteller bzw. Generalimporteure), Elektrizitätswirtschaft (VSE, Axpo/BKW/Alpiq, EVU und Gemeindewerke), Verbände/ NGO (TCS usw.).

\section{Hardware: Infrastruktur, Tankstellen, Treibstoff, Normen}

\begin{tabular}{|c|c|}
\hline Handlungsbedarf & Massnahme \\
\hline $\begin{array}{l}\text { Sichere und effiziente Nutzung der } \\
\text { Verkehrsinfrastruktur }\end{array}$ & $\begin{array}{l}\text { Bund: Schaffung rechtlicher Grund- } \\
\text { lagen für die Erfassung von Bewe- } \\
\text { gungsprofilen zur optimierten Nut- } \\
\text { zung von Infrastruktur und Verkehrs- } \\
\text { systemen. }\end{array}$ \\
\hline $\begin{array}{l}\text { Ergänzung/Aufbau der Strukturen zur } \\
\text { Produktion der zusätzlich benötigten } \\
\text { Energie }\end{array}$ & $\begin{array}{l}\text { Bund, Kantone, Gemeinden, Elektri- } \\
\text { zitätsbranche: Vorbildrolle. } \\
\text { Bund: Differenzierung Einspeisever- } \\
\text { gütung nach Eigenverbrauch analog } \\
\text { zu D (nicht einspeisende Fotovoltaik } \\
\text { mit Elektroauto als Speicher). }\end{array}$ \\
\hline $\begin{array}{l}\text { Infrastruktur zur Beladung der Fahrzeuge } \\
\text { bereitstellen }\end{array}$ & $\begin{array}{l}\text { Elektrizitätsbranche: Information } \\
\text { Bauherren (ggfs. Förderung), dass } \\
\text { Hausanschluss auf Hoch-Ampère } \\
\text { ausgelegt wird (vor allem bei Häu- } \\
\text { sern mit Tiefgaragen). } \\
\text { Elektrizitätsbranche, Autobranche: } \\
\text { Leitfaden für die Erstellung privater } \\
\text { oder öffentlicher Ladestationen. } \\
\text { ASTRA: E-Tankstellen-Masterplan } \\
\text { für Autobahnen; evtl. in Kooperation } \\
\text { mit Kantonen auch für Hauptstras- } \\
\text { sennetz. }\end{array}$ \\
\hline
\end{tabular}


Standardisierung bzw. Normierung insbesondere der Stecker, aber auch von Ladestationen und Abrechnungsmodellen

Lokale Stromnetze

Öffentlicher Verkehr
- $\quad$ Bund, KTU, Kantone, Gemeinden:

Bei jedem Umbau eines öffentlichen oder SBB-Parkplatzes ein Leerrohr verlegen (für spätere Ladestation).

- Kompatible Lösungen hinsichtlich des mechanischen Anschlusses; Einbindung der Fahrzeuge im Versorgungsnetz (z.B. Ladestationen und Abrechnungsmodelle).

- Elektrizitätsbranche, Bund: Teilnahme an Normierungsgremien sichern, entsprechende Gremienarbeit finanzieren.

- Gemeinde, Elektrizitätsbranche: Hochampère-Anschlüsse bei Haussanierungen vorsehen (inkl. Absicherung des Netzes).

- Kantone, Gemeinden: Lademöglichkeit in Bauvorschriften.

- Gemeinde, Elektrizitätsbranche: Gewährleisten, dass EVU auch bei grösseren Mengen an Elektroautos noch der «Anschlusspflicht» nachkommen können.

- Kantone, konzessionierte Transportunternehmen (KTU): Kombiangebot ÖV-Elektroauto schaffen.

- KTU: Steigerung der Energieeffizienz im ÖV (pro pkm), um den kompetitiven Vorsprung gegenüber MIV nicht zu verlieren. 


\begin{tabular}{|c|c|}
\hline Energy Contracting & $\begin{array}{l}\text { Elektrizitätsbranche: BHKW in Sied- } \\
\text { lungen zur Wärmeerzeugung und } \\
\text { Stromerzeugung auch für Elektromo- } \\
\text { bilität (Vorteil Speicherfähigkeit). } \\
\text { - Elektrizitätsbranche: Geschäftsmo- } \\
\text { dell zur Kopplung EV-Leasing mit } \\
\text { PV-Anlage z.B. auf Garagendach. }\end{array}$ \\
\hline $\begin{array}{l}\text { Weiterentwicklung und Anwendung } \\
\text { Verkehrstelematik }\end{array}$ & $\begin{array}{l}\text { Kantone: Zusammenarbeit mit Pri- } \\
\text { vatwirtschaft bei Bereitstellung von } \\
\text { Informationen, Kartenmaterial und } \\
\text { Echtzeitinformationen zu ÖV-Um- } \\
\text { steigepunkten, Umsteigerelationen, } \\
\text { Ladestationen und Car Sharing. }\end{array}$ \\
\hline Finanzierung Verkehrsinfrastruktur & $\begin{array}{l}\text { Bund: Einnahmen kilometer- und } \\
\text { primärenergie-effizienzabhängig } \\
\text { gestalten und damit unabhängig von } \\
\text { Antrieben und Treibstoffen. }\end{array}$ \\
\hline Wertewandel, Verkehrsplafonierung & $\begin{array}{l}\text { Kantone: Rechtliche Grundlagen für } \\
\text { Verwendung öffentlicher Parkplätze } \\
\text { durch Car-Sharing-Fahrzeuge. }\end{array}$ \\
\hline $\begin{array}{l}\text { Normierung von Steckern zur Schnell- } \\
\text { ladung }\end{array}$ & $\begin{array}{l}\text { Bund: Festlegung von Normen für } \\
\text { Schnittstellen unter dem Aspekt der } \\
\text { internationalen Kompatibilität. }\end{array}$ \\
\hline $\begin{array}{l}\text { Betankungsinfrastruktur bereitstellen, } \\
\text { Wartung Fahrzeuge sicherstellen }\end{array}$ & $\begin{array}{l}\text { ASTRA: Masterplan E-Tankstellen } \\
\text { zwecks Sicherstellung der Investitions- } \\
\text { sicherheit für die private Finanzierung } \\
\text { von E-Tankstellen entlang des Natio- } \\
\text { nalstrassennetzes. } \\
\text { - Erdölvereinigung: Koordination } \\
\text { E-Tankstellen im Tankstellennetz. } \\
\text { Autogewerbe: Wartung von Fahr- } \\
\text { zeugen mit Hochvolttechnik. }\end{array}$ \\
\hline
\end{tabular}




\begin{tabular}{|c|c|}
\hline \multicolumn{2}{|c|}{ Angebotsseite (Fahrzeuge) } \\
\hline Handlungsbedarf & Massnahme \\
\hline Erhöhung der Effizienz & $\begin{array}{l}\text { Bund: Regulierung des maximal } \\
\text { zulässigen Primärenergiever- } \\
\text { brauchs von Motorfahrzeugen } \\
\text { (jährliche Anpassung an tech- } \\
\text { nischen Fortschritt). } \\
\text { - } \\
\text { Mitarbeit/Übernahme Regelwerke } \\
\text { auf EU-Ebene (Weiterführung } \\
\mathrm{g} \mathrm{CO}_{2} / \mathrm{km} \text {-Zielwerte nach 2020). }\end{array}$ \\
\hline Weiterentwicklung Technik & $\begin{array}{l}\text { Bund mit Autobranche: Förderung } \\
\text { des Einsatzes von Leichtlaufölen } \\
\text { und reibungsarmen Reifen } \\
\text { (Zielvereinbarungen, Energiebera- } \\
\text { tungen). }\end{array}$ \\
\hline Neue Geschäftsmodelle & $\begin{array}{l}\text { - Autobranche: Mobility-Car-Sharing } \\
\text { komplementäre Angebote, analog } \\
\text { Car2Go (Mercedes) usw. }\end{array}$ \\
\hline
\end{tabular}

Nachfrageseite: Akzeptanz und Marktpenetration

\begin{tabular}{|l|l|}
\hline Handlungsbedarf & Massnahme \\
\hline Monetäre Anreize & $\begin{array}{l}\text { Gratisparkplätze für Elektroautos } \\
\text { und/oder -zweiräder in Siedlungs- } \\
\text { nähe und bei ÖV-Knoten. }\end{array}$ \\
$\begin{array}{l}\text { Steigerung Akzeptanz } \\
\text { effizienter Fahrzeuge }\end{array}$ & $\begin{array}{l}\text { Bund: Evaluationsverfahren und } \\
\text { Konsumenteninformation zu Energie- } \\
\text { verbrauch und Umweltbelastung von } \\
\text { Strassenfahrzeugen im Alltag } \\
\text { (LCA-Ansatz). }\end{array}$ \\
\hline
\end{tabular}




\begin{tabular}{|c|c|}
\hline & $\begin{array}{l}\text { - Bund: Analog zu U.S. EPA bei } \\
\text { Neuwagen auch Realverbrauch } \\
\text { angeben lassen. }\end{array}$ \\
\hline $\begin{array}{l}\text { Gewährleistung eines nachhaltigen } \\
\text { Strommixes }\end{array}$ & $\begin{array}{l}\text { Bund, Elektrizitätsbranche: } \\
\text { Garantie einer E-Mobilitäts-Strom- } \\
\text { mix-Mindestqualität } \\
\text { - EVU und Autobranche: Neue } \\
\text { Geschäftsmodelle; Kopplung } \\
\text { Strommix und Autoleasing; Fahrzeug } \\
\text { im Eigentum des EVU («Mobility } \\
\text { Energy Contracting»). }\end{array}$ \\
\hline Freizeitverkehr & $\begin{array}{l}\text { - Bund, Tourismus, NGO: Demons- } \\
\text { trationsprojekte gezielt für Freizeit- } \\
\text { verkehr (inkl. Berggebiet). }\end{array}$ \\
\hline
\end{tabular}

Monetäre Aspekte: Finanzierung, Anreize und Subventionen

\begin{tabular}{|l|l|}
\hline Handlungsbedarf & Massnahme \\
\hline Kertewandel, Verkehrsplafonierung & $\begin{array}{l}\text { Bund, Kantone: Änderung Lohnsteu- } \\
\text { erabzüge (Eliminierung der Subventi- } \\
\text { onierung des Pendlerverkehrs). }\end{array}$ \\
. $\begin{array}{l}\text { Bund: Regressive Elemente in die } \\
\text { kilometerabhängige Besteuerung zur } \\
\text { Reduktion kurzer (substituierbarer) } \\
\text { Fahrten: Sockelgebühr für die ersten } \\
\text { Kilometer (ausser für erste/letzte } \\
\text { Meile hin zu ÖV-Knoten). }\end{array}$ \\
\\
\end{tabular}


Steigerung Akzeptanz effizienter Fahrzeuge
Anreizsysteme
- $\quad$ Prüfung der Förderung unabhängiger Batterievermietgesellschaften, Second-Use-Batterie-Weiterverwendungsgesellschaften, CarsharingOrganisationen.

- Bund und Kantone: Einführen von Anreizsystemen beim Autokauf auf Basis der Energieetikette oder ähnlicher Kategorisierungsverfahren, finanziert z.B. über Automobilsteuer, zeitlich limitiert.

- Kantone: Differenzierung kant. Motorfahrzeugsteuern (BonusMalus-Systeme) nach Energieverbrauch, Harmonisierung der Bemessungsgrundlagen.

- $\quad$ Bund, NGO: Informationsmassnahmen zur Rolle des Neuwagenkaufs und zu Energieeffizienzpotenzialen.

- Bund, Kantone/KKFD: Fiskalische Massnahmen wie verbrauchsabhängige Differenzierung der Steuerwirksamkeit von Lohnnebenleistungen wie Geschäftsautos.

- Kantone: Einmalig ausbezahlter hoher Bonus für Elektrofahrzeuge (nur in Anfangsphase).

- $\quad$ Bund: Bei Anreizsystemen Prüfung von technologiespezifischen Bonusprämien für Elektrofahrzeuge (zeitlich befristet) («Super-bonus»). 


\begin{tabular}{|l|l|}
\hline Internalisierung externer Kosten & $\begin{array}{l}\text { Bund, Kantone: Well-to-wheel-Ener- } \\
\text { gieaufwendung als Bemessungs- } \\
\text { grundlage (kilometerabhängige } \\
\text { Besteuerung von Primärenergie- } \\
\text { effizienz abhängig machen, } \\
\text { Effizienzvorschriften). }\end{array}$ \\
Besteuerung MIV & $\begin{array}{l}\text { Bund: } \mathrm{CO}_{2} \text {-Abgabe auf Treibstoffe } \\
\text { einführen. }\end{array}$ \\
& $\begin{array}{l}\text { Kantone, Bund: Grundlagen für } \\
\text { Harmonisierung kant. Steuermodelle } \\
\text { (allfällige kilometerabhängige Be- } \\
\text { steuerung soll eine jährliche Grund- } \\
\text { steuer nicht ersetzen, auch zwecks } \\
\text { Reboundvermeidung; Kleinstfahrzeu- } \\
\text { ge, welche zulassungsrechtlich als } \\
\text { Motorräder oder Kleinmotorwagen } \\
\text { gelten, wie kleine PKW besteuern). }\end{array}$ \\
\hline & \\
\hline
\end{tabular}

\section{Forschungsbedarf}

\begin{tabular}{|c|c|}
\hline Handlungsbedarf & Massnahmen \\
\hline Wertewandel & $\begin{array}{l}\text { Alle Akteure: Erforschen, wie über } \\
\text { Kommunikation, Best Practices, } \\
\text { Gruppen-Selbstverpflichtungen usw. } \\
\text { der Wertewandel herbeigeführt und } \\
\text { verstärkt werden kann. } \\
\text { - Forschung zu sozioökonomischer } \\
\text { Innovation (andere Mobilitätskonzep- } \\
\text { te, Änderung des Nutzerverhaltens). } \\
\text { Forschung zu Batterietechnik und } \\
\text {-management. }\end{array}$ \\
\hline
\end{tabular}




\begin{tabular}{|l|l|}
\hline Grundlagen & $\begin{array}{l}\text { Bund: Erforschung des Strommixes } \\
\text { (gemittelt und marginal) für MIV- } \\
\text { Elektrizität (tagesganggewichtet), } \\
\text { Möglichkeiten des Einsatzes } \\
\text { marginaler Strommixe für EV bei } \\
\text { energiepolitischen Instrumenten. }\end{array}$ \\
Second-Life-Batterien & $\begin{array}{l}\text { Bund: Geschäftsmodelle für Second- } \\
\text { Life-Einsatz. } \\
\text { Bund: Erforschung von Recycling } \\
\text { und Second-Life von Batterien }\end{array}$ \\
inkl. Entfernung rechtlicher Hin-der- \\
nisse für neue Geschäfts-modelle.
\end{tabular}




\section{A1 Literaturverzeichnis}

Althaus, H.J., DelDuce, A., et al. (2012). Life cycle Assessment of mobility requirements of the Swiss Post. Internal Report. Bern, Switzerland, Die Post.

Althaus, H.J., Gauch, M. (2010). Vergleichende Ökobilanz individueller Mobilität. Elektromobilität versus konventionelle Mobilität mit Bio- und fossilen Treibstoffen. Dübendorf, Empa.

Andersson, B.A. (2001). Material constraints on technology evolution: the case of scarce metals and emerging energy technologies. Department of Physical Resource Theory. Göteborg, Sweden, Chalmers University of Technology and Göteborg University. Doctor of Philosophy: 67.

Andersson, B.A., Rade, I. (1999). Large-scale electric-vehicle battery systems: long-term metal resource constraints, Chalmers, Göteborg University: 36.

Andersson, B.A., Rade, I. (2001). Metal resource constraints for electric-vehicle batteries. Transportation Research Part D-Transport and Environment 6(5): 297-324.

Anderson, D. L. (2009). An Evaluation of current and future costs for Lithium-lon batteries for use in electrified vehicle powertrains. Master of Environmental Management, Nicholas School of the Environment of Duke University.

Angerer, G., Erdmann, L., Marscheider-Weidemann, F., Scharp, M., Lüllmann, A., Handke, V., Marwede, M. (2009a). Rohstoffe für Zukunftstechnologien. Stuttgart.

Angerer, G., Marscheider-Weidemann, F., Wendl, M., Wietschel, M. (2009b). Lithium für Zukunftstechnologien Nachfrage und Angebot unter besonderer Berücksichtigung der Elektromobilität. Fraunhofer ISI, Karlsruhe.

APS Panel (2009). Energy Critical Elements. Securing Materials for Emerging Technologies. A report by the APS Panel on Public Affairs \& the Materials Research Society.

ARE (2006). Perspektiven des Schweizerischen Personenverkehrs 2030. Bundesamt für Raumentwicklung (ARE), Bern. 
asa (2011). Künftige Entwicklung der Personenwagen-Neuzulassungen in der Schweiz. Szenarien zur Entwicklung von Treibstoffverbrauch, CO2-Emissionen, Leer- und Gesamtgewicht, Hubraum und Leistung bis 2030. EBP in Auftrag der asa - Vereinigung der schweizerischen Strassenverkehrsämter. Schlussbericht vom 10. März 2011, 58 Seiten.

ASTRA (2003). NISTRA: Nachhaltigkeitsindikatoren für Strasseninfrastrukturprojekte. Ein Instrument zur Beurteilung von Strasseninfrastrukturprojekten unter Berücksichtigung der Nachhaltigkeitsziele. Bundesamt für Strassen (ASTRA), Methodenbericht, 25. August 2003.

Bach, Ch. (2008). Challenges and Potential of advanced methane gas vehicles.

SATW Congress - Mobility 2030. Yverdon.

Bach, Ch. Umfangreiche Untersuchungen auf den Prüfständen der Empa Dübendorf zum Wirkungsgrad von aktuellen Fahrzeugmodellen unter verschiedensten Testbedingungen. Publikationen in Vorbereitung.

Bae, J.C. (2010). Strategies and Perspectives for Securing Rare Metals in Korea. Critical Elements for New Energy Technologies. Proceedings of the Workshop, Boston, MA, Apr 29, 2010. Cambridge, MA.

Bae, J.C. (2010). Strategies and Perspectives for Securing Rare Metals in Korea. Critical Elements for New Energy Technologies. Proceedings of the Workshop, Boston, MA, Apr 29, 2010, Cambridge, MA.

BAFU (2012). Emissionen nach $\mathrm{CO}_{2}$-Gesetz und Kyoto-Protokoll. Letzte Aktualisierung: 06.07.2012.

Bauer, C., Simons, A. (2010). Ökobilanz der Elektromobilität - Analyse der e-Twingos der EKZ. Villingen, PSI.

BCG (2010). Batteries for Electric Cars: Challenges, Opportunities, and the Outlook to 2020, Boston Consulting Group (BCG).

Becker, A.T. (2009). Electric Vehicles in the United States. A New Model with Forecasts to 2030. Center for Entrepreneurship \& Technology (CET). Technical Brief, Berkeley.

BFE (2012a). Schweizerische Gesamtenergiestatistik 2011. 
BFE (2012b). Die Energieperspektiven für die Schweiz bis 2050. Energienachfrage und Elektrizitätsangebot in der Schweiz 2000-2050. Ergebnisse der Modellrechnungen für das Energiesystem. Prognos AG in Auftrag Bundesamt für Energie, Basel, 12. Sept. 2012.

BFS (2010a). Bundesamt für Statistik, Medienmitteilung vom 30. Juli 2010.

BFS (2010b). Szenarien zur Bevölkerungsentwicklung der Schweiz 2010-2060. Neuchâtel, 2010.

BFS (2012). Mobilität in der Schweiz. Ergebnisse des Mikrozensus Mobilität und Verkehr 2010. Bundesamt für Statistik, Neuenburg.

Blaser, F., Castelanelli, S., Wäger, P., Widmer, R. (2012). Seltene Metalle in Elektro- und Elektronikaltgeräten - Vorkommen und Rückgewinnungstechnologien; Empa St. Gallen: Ittigen.

Boulanger, A.G., Chu, A.C., Maxx, S., Waltz, D.L. (2011). Vehicle Electrification: Status and Issues. Proceedings of the IEEE 99.

Buchman, I. (2005). «BatteryUniversity.com.» Retrieved 10.6.2012, from www.batteryuniversity.com.

Buller, U., Marscheider-Weidemann, F. Wietschel, M. (2012). Alternativen bei drohender Rohstoffknappheit. Zeitschrift für Automobilwirtschaft 2/2012.

Bundesrat (2012). Erste Massnahmen Energiestrategie 2050. Faktenblatt 1 vom 18.04.12, (http://www.news.admin.ch/NSBSubscriber/message/attachments/26592.pdf).

Carles, L. (2010). Modelling long-term global lithium stocks and flows, an energy perspective in the context of E-mobility transition. Master thesis. EPFL/Empa/Universite de Lausanne.

Chau, K.T., Chan, C.C., Liu, C. (2008). Overview of permanent-magnet brushless drives for electric and hybrid electric vehicles. Transactions on Industrial Electronics 55(6): 2246-2257. 
de Haan P., Mueller M.G., Peters A. (2006a). Does the hybrid Toyota Prius lead to rebound effects? Analysis of size and number of cars previously owned by Swiss Prius buyers. Ecological Economics, 58, 592-605.

de Haan P., Peters A., Mueller M.G. (2006b). Comparison of Buyers of Hybrid and Conventional Internal Combustion Engine Automobiles: Characteristics, Preferences, and Previously Owned Vehicles. Transportation Research Records, 1983, 106-113.

de Haan P., Peters, A., Scholz, R.W. (2007). Reducing energy consumption in road transport through hybrid vehicles: Investigation of rebound effects, and possible effects of tax rebates. Journal of Cleaner Production, 15, 1076-1084.

de Haan, P. (2009). Energieeffizienz und Reboundeffekte: Entstehung, Ausmass, Eindämmung. Bundesamt für Energie, Programm Energiewirtschaftliche Grundlagen. ETH Zürich, IED-NSSI, report EMDM1524.

de Haan, P. (2011). Elektromobilität - wer profitiert und wer bezahlt? Szenarien zur Marktdurchdringung und Mineralölsteuererträge. Vortrag am 2. Schweizer Forum Elektromobilität, Verkehrshaus Luzern, 26. Januar 2011.

de Haan, P., Keller, M. (2000). Emission factors for passenger cars: application of instantaneous emission modeling. Atmospheric Environment, 34, pp. 4629-4638.

de Haan, P., Mueller, M.G., Peters, A. (2006). Does the hybrid Toyota Prius lead to rebound effects? Analysis of size and number of cars previously owned by Swiss Prius buyers. Ecological Economics, 58, 592-605.

de Haan, P., Mueller, M.G., Peters, A., Hauser, A. (2007). Lenkungsabgaben zur Senkung des CO2-Ausstosses der PKW-Neuzulassungen: Hintergrund, Mechanismen, Prognosen. Schlussbericht. Programm Energiewirtschaftliche Grundlagen, Swiss Federal Agency for Energy, Bern.

de Haan, P., Mueller, M.G., Scholz, R.W. (2009). How much do incentives affect car purchase? Agent-based microsimulation of consumer choice of new cars, part II: Forecasting effects of feebates based on energy-efficiency. Energy Policy, 37, 1083-1094.

Deutsche-Bank (2010). The End of the Oil Age: 2011 and beyond: a reality check. D. C. P. Sankey, S. Micheloto: 59. 
Duleep, G., van Essen, H., Kampman, B., Grünig, M. (2011). Impacts of Electric Vehicles - Deliverable 2. Assessment of electric vehicle and battery technology. Delft, CE Delft.

Dunn, J., Gaines, L., Sullivan, J., Wang, M. (2012). Impact of Recycling on Cradle-to-Gate Energy Consumption and Greenhouse Gas Emissions of Automotive Lithium-Ion Batteries. Argonne National Lab, Illinois USA, es302420z Environmental Science and Technology 2012, 46, 12704-12710.

EBP (2008). Wie weiter mit dem Verkehr? Strategien zur Verbesserung der Zürcher Mobilität. Langfassung zum Bericht der Zürcher Kantonalbank. Zürich, 17. April 2008.

EBP (2009). Risikoabschätzung zu alternativen Treibstoffen und Antrieben. Möglicher Einfluss auf die Risiken des Strassenverkehrs durch Einsatz neuer Antriebskonzepte. Im Auftrag des Bundesamts für Energie, Bern.

Empa (2010). Materialflüsse der elektrischen und elektronischen Geräte in der Schweiz. Im Auftrag des BAFU, Bern.

Envia (2012). Press release 27.02.2012 by Envia Systems (enviasystems.com) announcing $400 \mathrm{Wh} / \mathrm{kg}$ at cell level for a 45Ah (C/3) automotive grade cell with over 300 cycles for an estimated cost of USD 125.-/kWh. Si-C anode, high capacity manganese rich HCMR cathode and high voltage EHV electrolyte (4.6V standard charge voltage).

Erdmann, L., Graedel, T.E. (2011). The Criticality of Non-Fuel Minerals: A Review of Major Approaches and Analyses. Environmental Science \& Technology 45:7620-7630.

European Commission (2010). Critical Raw Materials for the EU. Report of the Ad-hoc Working Group on defining critical raw materials.

Forbes (2012). Will the electric car be closer to mass market? Forbes India Magazine. January 2012.

Fraunhofer ISI (2009). Lithium für Zukunftstechnologien. Nachfrage und Angebot unter besonderer Berücksichtigung der Elektromobilität. Karlsruhe.

Fraunhofer ISI und IZT (2009). Rohstoffe für Zukunftstechnologien: Einfluss des branchenspezifischen Rohstoffbedarfs in rohstoffintensiven Zukunftstechnologien auf die zukünftige Rohstoffnachfrage. Schlussbericht vom 15. Mai 2009 in Auftrag des Bundesministeriums für Wirtschaft und Technologie. Stuttgart. 
Fraunhofer (2011). Produkt Roadmap Lithium-Ionen-Batterien 2030. F. I. f. S.-u. Innovationsforschung: 28.

Frischknecht, R. (2012). Umweltaspekte von Elektroautos. Uster, ESU-services.

Frischknecht, R., Jungbluth, N. et al. (2004). Overview and Methodology. Dübendorf, CH, Swiss Centre for Life Cycle Inventories.

Gasche U.P., Guggenbühl H.P., 2010. Schluss mit dem Wachstumswahn. Plädoyer für eine Umkehr. Rüegger Verlag, Glarus/Chur 2010.

Geringer, B., Tobler K. (2012). Batterieelektrische Fahrzeuge in der Praxis. Wien, Österreichischer Verein für Kraftfahrzeugtechnik (ÖVK) und Österreichischer Automobil-, Motorrad- und Touring Club (ÖAMTC).

Giannouli, M., de Haan, P., Keller, M., Samaras, Z., (2007). Waste from Road Transport: Development of a model to predict waste from end-of-life and operation phases of road vehicles in Europe. Journal of Cleaner Production, 15, 1169-1182.

Giannouli, M., Samaras, Z., Keller, M., de Haan, P., Kallivoda, M., Sorenson, S., Georgakaki, A., (2006). Development of a database system for the calculation of indicators of environmental pressure caused by transport. Science of the Total Environment, 357, 247-270.

Girod, B., de Haan, P., Scholz, R.W. (2010). Consumption-as-usual instead of ceteris paribus assumption for demand. Integration of potential rebound effects into LCA. Int $J$ Life Cycle Assess (2011) 16:3-11, DOI 10.1007/s11367-010-0240-z.

Girod, B., de Haan, P. (2009). GHG reduction potential of changes in consumption patterns and higher quality levels: Evidence from Swiss household consumption survey. Energy Policy, 37, 5650-5661, doi:10.1016/j.enpol.2009.08.026.

Girod, B., de Haan, P. (2010). More or better? A model for changes in household greenhouse gas emissions due to higher income. Journal of Industrial Ecology, 14(1), 31-49, DOI: 10.1111/j.1530-9290.2009.00202.x.

Goedkoop M., Heijungs R., Huijbregts M., De Schryver A., Struijs J., van Zelm R., ReCi$\mathrm{Pe}$ 2008. A life cycle impact assessment method which comprises harmonised category indicators at the midpoint and the endpoint level. First edition. Report I: Characterisation. 6 January 2009. 
Graedel, T.E., Barr, R., Chandler, C., Chase, T., Choi, J., Christoffersen, L., Friedlander E., C. Henly, Jun, C., Nassar, N.T., Schechner, D., Warren, S., Yang, M.Y., Zhu, C. (2011). Methodology of Metal Criticality Determination. Environmental Science \& Technology 46:1063-1070.

Grünig, M., Witte, M., Marcellino, D., Selig, J., van Essen, H. (2011). Impacts of Electric Vehicles - Deliverable 1: An overview of Electric Vehicles on the market and in development. Delft, ICF International, Ecologic Institute.

Guardian (2011). China plans to make a million electric vehicles a year by 2015. Guardian Environment Network.

Habermacher, F. (2011). Modeling Material Inventories and Environmental Impacts of Electric Passenger Cars - Comparison of LCA results between electric and conventional vehicle scenarios. Dep. of Environmental Sciences, ETH Zurich. Masterarbeit.

Hatch, G.P. (2011). Critical Rare Earths - Global supply \& demand projections and the leading contenders for new sources of supply., TMR Technology Metals Research.

IEA (2010). Energy Technology Perspectives, Scenarios \& Strategies to 2050, Executive Summary.

IEA (2011). World Energy Outlook 2011.

IPCC (2007). Climate Change 2007: The Physical Science Basis. Contribution of Working Group I to the Fourth Assessment Report of the Intergovernmental Panel on Climate Change. S. Solomon, D, M. Qin, Z. Manning, et al.; Cambridge University Press.

ISO 14‘040 (2006). Environmental Management - Life Cycle Assessment - Principles and Framework. Brussels, ISO/TC 207/SC5.

ISO 14‘044 (2006). Environmental Management - Life Cycle Assessment - Requirements and guidelines. Brussels, ISO/TC 207/SC5.

Jung, H.-G., Hassoun, J., Park, J.-B., Sun, Y.-K., Scrosati, B. (2012); An improved highperformance lithium-air battery; Nature Chemistry 4, 579-586; doi:10.1038/nchem.1376, http://7ms.com/abt/online/2010/10/dry-rooms.html.

Le Bris, J. (2011). Das Elektro-Rad bzw. Pedelec als neues Verkehrsmittel. Nutzerprofile 
und Einsatzpotenziale. PowerPoint-Präsentation anlässlich der Jahrestagung 2011 des AK Verkehr der DGfG in Tübingen am 21. Juli 2011. Universität Tübingen.

McKinsey (2009). Swiss Greenhouse Gas Cost Abatement Curve.

McKinsey (2011). (Russell Hensley et al.): The fast lane to the adoption of electric cars, McKinsey Quarterly Feb 2011.

Morley, N. (2008). Material Security. Ensuring resource availability to the UK economy. Chester, UK, Oakedene Hollins; C-Tech Innovation.

Mueller, M.G., de Haan, P. (2009). How much do incentives affect car purchase? Agentbased microsimulation of consumer choice of new cars, part I: Model structure, simulation of bounded rationality, and model validation. Energy Policy, 37, 1072-1082.

Nassar, N.T., Barr, R., Browning, M., Diao, Z., Friedlander, E., Harper, E. M., Henly, C., Kavlak, G., Kwatra, S., Jun, C., Warren, S., Yang, M.Y., Graedel, T. E. (2011). Criticality of the Geological Copper Family. Environmental Science \& Technology 46:1071-1078.

National Research Council (2008). Minerals, Critical Minerals and the U.S. Economy. The National Academies Press, Washington D.C.

Neudorfer, H. (2009). Elektrische Antriebe in Fahrzeugen - «die» Lösung für die Zukunft?, Technische Universität Darmstadt.

Notter, D.A., Gauch, M., Widmer, R., Wager, P., Stamp, A., Zah, R., Althaus, H.J. (2010). Contribution of Li-Ion Batteries to the Environmental Impact of Electric Vehicles. Environmental Science \& Technology 44(17): 6550-6556.

NPE (2011). Electromobility - International plug standard for customers, DIN Deutsches Institut für Normung e. V., Nationale Plattform Elektromobilität - Work Group 4 «Standardisation and Certification».

OICA (2011). http://oica.net/category/production-statistics/ [Juli 2012].

Pautzke, R. (2010). Radnabenantriebe: Studie zur Abschätzung der Verfügbarkeit des Entwicklungsstandes und des Potentials von Radnabenantrieben in der Elektromobilität. Aachen, Shaker Verlag. 
Pfleger, P., Lichtblau, K., Bardt, H., Reller, A. (2009). Rohstoffsituation Bayern: Keine Zukunft ohne Rohstoffe. Strategien und Handlungsoptionen. I. C. V. d. B. Wirtschaft. München.

Polyplus (2011). http://www.polyplus.com/liair.html.

Rade, I., Andersson, B.A. (2001). Requirement for metals of electric vehicle batteries. Journal of Power Sources 93(1-2): 55.

Schweimer, G.W., Levin, M. (2002). Life Cycle Assement for Golf A4. Wolfsburg, Volkswagen $A G$.

Shinko Research (Mitsubishi UFJ Research and Consulting) (2009). Trend Report of Development in Materials for Substitution of Scarce Metals. New Energy and Industrial Technology Development Organization (NEDO), Tokyo.

Sion (2011). High Energy Rechargeable Li-S Cells for EV Application. Status, Challenges and Solutions. Presentation of Sion Power at Sion Power for the Electrochemical Society (http://sionpower.com/pdf/articles/SionPowerECS.pdf).

SMSP (2008). Evidence and Analytic Support. Institute for Defense Analyses (IDA), Alexandria, VA.

Spielmann, M., de Haan, P. (2008). Umweltindikatoren im Verkehr - Vergleich der Verkehrsmittel anhand CO2-Emissionen, Energieaufwand und übriger Umweltauswirkungen. Rüegger Verlag, Zürich.

Spielmann, M., de Haan, P., Scholz, R.W. (2008). Environmental Rebound Effects of High-Speed Transport Technologies: A case study of climate change rebound effects of a future underground maglev train system. Journal of Cleaner Production, Vol. 16, 13881398.

Spielmann, M., Scholz, R.W., Tietje, O., de Haan, P. (2005). Scenario Modelling in Prospective LCA of Transport Systems: Application of Formative Scenario Analysis. Int. J. Life-Cycle Assessment, 10, Nr. 5, pp. 325-335.

Stamp, A., Lang, D.J., Wäger, P.A. (2012). Environmental impacts of a transition toward e-mobility: The present and future role of lithium carbonate production. Journal of Cleaner Production. 23(1): 104-112. 
Strasseschweiz (2012). Vademecum.

TA-SWISS (Hrsg.) (2010). Rainer Zah et. al: Future Perspectives of 2nd Generation Biofuels, vdf Hochschulverlag AG an der ETH Zürich und Kurzfassung www.ta-swiss.ch.

TCS (2012). Kosten eines Musterautos 2012.

THELMA (2012). Technology-centered Electric Mobility Assessment. Retrieved 5. Mar 2012, www.thelma-emobility.net/index.html.

Thomason, J.S., Atwell, R.J., et al. (2008). From National Defense Stockpile (NDS) to Strategic Materials Security Programme (SMSP): Evidence and Analytic Support. Alexandria, VA, Institute for Defense Analyses (IDA). 1.

U.S. DOE (2011). Critical Materials Strategy. United States Department of Energy.

UNEP (2010). Assessing the Environmental Impacts of Consumption and Production: Priority Products and Materials, A Report of the Working Group on the Environmental Impacts of Products and Materials to the International Panel for Sustainable Resource Management. Hertwich, E., van der Voet, E., Suh, S., Tukker, A., Huijbregts M., Kazmierczyk, P., Lenzen, M., McNeely, J., Moriguchi, Y.

UNEP (2011). Recycling rates of metals - a status report. A report of the Working Group on the Global Flows to the International Resource Panel. Graedel, T.E., Allwood, J., Birat, J.P., Reck, B.K., Sibley, S.F., Sonnemann, G., Buchert, M., Hagelüken, C.

USGS (2012). http://minerals.usgs.gov/minerals/pubs/mcs/2012/mcs2012.pdf [30.7.2012].

UVEK (2008). Ziel- und Indikatorensystem nachhaltiger Verkehr UVEK (ZINV UVEK), Version Oktober 2001, aktualisiert 2008 (http://www.are.admin.ch/dokumentation/ publikationen/ 00024/00283/index.html?lang=de).

Wäger, P.A. (2011). Scarce metals - Applications, supply risks and need for action. Notizie di Politeia 27:57-66.

Wäger, P.A., Hischier, R., Eugster, M. (2011b). Environmental impacts of the Swiss collection and recovery systems for Waste Electrical and Electronic Equipment (WEEE): A follow-up. Science of The Total Environment In Press, Corrected Proof. 
Wäger, P., Widmer, R., Stamp, A. (2011a). Scarce technology metals - applications, criticalities and intervention options. Empa, St. Gallen.

Wäger, P.A., Lang, D.L., Bleischwitz, R., Hagelüken, C., Meissner, S., Reller, A., Wittmer, D. (2010). Seltene Metalle - Rohstoffe für Zukunftstechnologien. Swiss Academy of Engineering Sciences (SATW), Zürich.

Wang, L.J., Li, J.Q., Li, S.H., Zhang, G.Q., Huang, S.L. (2011). Application of Amorphous Alloy in the New Energy-Efficient Electrical Motor. Applied Mechanics and Materials 4849: 246-248.

Weilenmann, M.F., Alvarez, R., Keller, M. (2010). Fuel Consumption and CO2/Pollutant Emissions of Mobile Air Conditioning at Fleet Level - New Data and Model Comparison. Environmental Science \& Technology 44: 5277-5282.

Widmer, R., Gauch, M. (2012). Eigene Messungen.

Zackrisson, M., Avellán, L., Orlenius, J. (2010). Life cycle assessment of lithium-ion batteries for plug-in hybrid electric vehicles - Critical issues. Journal of Cleaner Production 18(15): 1519-1529.

Zah, R., Binder, C., Bringezu, S., Reinhard, J., Schmid, A., Schütz, H. (2010). Future Perspectives of 2nd Generation Biofuels. Zürich, vdf Hochschulverlag AG an der ETH Zürich. 


\section{A2 Glossar}

\begin{tabular}{|c|c|}
\hline Antriebsstrang (Drive Train) & $\begin{array}{l}\text { Teil des Fahrzeugs; beinhaltet Motor(en), Getriebe und } \\
\text { Kraftübertragung bis hin zum Rad. }\end{array}$ \\
\hline BEV (Battery Electric Vehicle) & $\begin{array}{l}\text { Fahrzeug, das ausschliesslich von einem batteriege- } \\
\text { speisten Elektromotor angetrieben wird. BEV können } \\
\text { nur mit Strom getankt werden. }\end{array}$ \\
\hline EV (Electriv Vehicle) & Siehe BEV. \\
\hline FCV (Fuel cell vehicle) & $\begin{array}{l}\text { Brennstoffzellenfahrzeug, welches mit Wasserstoff oder } \\
\text { Erdgas (im Fahrzeug zu Wasserstoff reformiert) betankt } \\
\text { wird. Mit dem Wasserstoff wird in einer Brennstoffzelle } \\
\text { Strom für den elektrischen Antrieb erzeugt. Überschüs- } \\
\text { siger Strom wird in Batterien zwischengespeichert. }\end{array}$ \\
\hline Glider & $\begin{array}{l}\text { Teil des Fahrzeugs; beinhaltet die Karosserie, Innenein- } \\
\text { richtung, Achsen, Aufhängungen, Räder und alle } \\
\text { weiteren Teile, die weder zum Antriebsstrang noch zum } \\
\text { Energiespeicher gehören. }\end{array}$ \\
\hline HEV & $\begin{array}{l}\text { Hybrid Electric Vehicle, eine Kombination aus Verbren- } \\
\text { nungsmotor und Elektromotor mit Batterie; ein HEV } \\
\text { tankt ausschliesslich Benzin oder Diesel (prinzipiell ist } \\
\text { auch Gas möglich), Strom wird nur intern durch den } \\
\text { Verbrennungsmotor oder durch Rekuperation erzeugt } \\
\text { und in der Batterie zwischengespeichert. Ein HEV kann } \\
\text { nicht an der Steckdose angeschlossen werden (siehe } \\
\text { PHEV). HEV fahren gar nicht (milder Hybrid) oder nur } \\
\text { kurze Strecken (Vollhybrid) rein elektrisch. }\end{array}$ \\
\hline ICE (Internal Combustion Engine) & $\begin{array}{l}\text { Verbrennungsmotor, resp. Fahrzeug, welches mit einem } \\
\text { Verbrennungsmotor angetrieben wird. Der Treibstoff } \\
\text { kann Diesel, Benzin, Erdgas oder Biotreibstoff sein. }\end{array}$ \\
\hline
\end{tabular}




\begin{tabular}{|c|c|}
\hline Hybridfahrzeug & $\begin{array}{l}\text { Fahrzeug, das einen kombinierten Antrieb, } \\
\text { bestehend aus Verbrennungsmotor und } \\
\text { Elektromotor, besitzt. Darunter fallen sehr } \\
\text { unterschiedliche Fahrzeugkonzepte von } \\
\text { Benzinfahrzeugen mit einem vergrösserten } \\
\text { Anlassermotor (> HEV mit milder Hybridisie- } \\
\text { rung) bis hin zu Elektrofahrzeugen, die auf } \\
\text { langen Fahrtstrecken On-Board-Strom mithilfe } \\
\text { eines Verbrennungsmotors produzieren } \\
\text { (> Range Extender). }\end{array}$ \\
\hline Kleinmotorwagen & $\begin{array}{l}\text { Eine Motorfahrzeug gemäss VTS-Verordnung. } \\
\text { Kleinmotorwagen unterliegen weniger strikten } \\
\text { Anforderungen als normale Personenwagen, } \\
\text { dürfen aber eine bestimmte Gewichtslimite } \\
\text { nicht überschreiten. Sie stellen ein Segment } \\
\text { der Gruppe der in dieser Studie «3-Wheeler» } \\
\text { genannten Fahrzeuge dar. Der Renault Twizy } \\
\text { ist ein aktuelles Beispiel. }\end{array}$ \\
\hline Kleinst-EV & $\begin{array}{l}\text { EV, welche nicht als «leichter Motorwagen» } \\
\text { im Sinne der VTS-Verordnung, sondern } \\
\text { zulassungsrechtlich als Motorfahrrad, Klein- } \\
\text { motorrad, Motorrad oder Kleinmotorwagen } \\
\text { gelten. Eine genaue, auch für die Zukunft } \\
\text { geltende Abgrenzung zwischen «E-Scooter» } \\
\text { und «Kleinst-EV» gibt es dabei aus Sicht des } \\
\text { Nutzers nicht. }\end{array}$ \\
\hline MIV & Motorisierter individueller Verkehr. \\
\hline NEDC, NEFZ & $\begin{array}{l}\text { Neuer europäischer Fahrzyklus (New Euro- } \\
\text { pean Driving Cycle, NEDC). }\end{array}$ \\
\hline
\end{tabular}




\begin{tabular}{|c|c|}
\hline ÖV & Öffentlicher Verkehr \\
\hline Paralleler Hybrid & $\begin{array}{l}\text { Ein Parallelhybrid ist ein Hybridfahrzeug, } \\
\text { bei welchem sowohl Elektromotor als auch } \\
\text { Verbrennungsmotor direkt mechanisch das } \\
\text { Rad antreiben. Elektromotor und Batterie } \\
\text { erlauben den on/off-Betrieb des Verbren- } \\
\text { nungsmotors und unterstützen bei Leistungs- } \\
\text { bedarfspitzen, sodass der Motor kleiner } \\
\text { ausgelegt werden kann und höhere thermo- } \\
\text { dynamische Effizienz erreicht. }\end{array}$ \\
\hline PHEV (Plug-in hybrid electric vehicle) & $\begin{array}{l}\text { Hybridfahrzeug, das sowohl mit flüssigem } \\
\text { Treibstoff betankt als auch mit Strom geladen } \\
\text { werden kann. PHEV haben elektrische Reich- } \\
\text { weiten von rund } 20 \text { bis } 150 \text { Kilometer und } \\
\text { benötigen den flüssigen Treibstoff für } \\
\text { Langstreckenfahrten. }\end{array}$ \\
\hline Range Extender & Siehe PHEV. \\
\hline Serieller Hybrid & $\begin{array}{l}\text { Ein Hybridfahrzeug, bei welchem ein eher } \\
\text { kleiner Verbrennungsmotor ausschliesslich für } \\
\text { die Stromerzeugung zuständig ist und keine } \\
\text { direkte mechanische Kraftübertragung vom } \\
\text { Verbrennungsmotor zum Rad besteht. Der } \\
\text { variierende Leistungsbedarf im dynamischen } \\
\text { Fahrbetrieb wird voll durch Elektromotor und } \\
\text { Batterie aufgefangen, welche grösser dimen- } \\
\text { sioniert werden müssen als bei parallelen } \\
\text { Hybriden. }\end{array}$ \\
\hline
\end{tabular}




\section{A3 Potenzial für Verlagerung von MIV-Fahrleistung hin zu Kleinst-EV (und Bahn)}

In diesem Anhang wird untersucht, wie gross - auf Basis der Daten des Mikrozensus Verkehr 2010 (ARE und BFS) - das Potenzial zur Änderung des Verkehrsverhaltens in Richtung einer stärker auf Intermodalität ausgerichteten motorisierten Personenmobilität ist. Solche Änderungen werden im dritten Szenario «COM - connected mobility» angenommen: Eine deutliche Steigerung der Fahrleistungsanteile der Kleinstwagenkonzepte.

Im COM-Szenario wird davon ausgegangen, dass ein Teil der heute mit Personenwagen erbrachten MIV-Fahrleistung neu mit Kleinst-EV (siehe Abbildung 53) zurückgelegt wird. Dies bedingt Änderungen im Mobilitätsverhalten: Vermehrt würden lange MIV-Fahrten durch Kombinationen von MIV und Bahn ersetzt. Als Instrumente zur Kombination der beiden Verkehrsträger sind Park+Ride $(P+R)$, Fahrzeugmiete, Carsharing sowie neue Geschäftsmodelle (Leasing eines Kleinst-EV für erste/letzte Meile von einer KTU in Kombination mit einem Generalabonnement und bahnhofnahem Parkieren) denkbar.
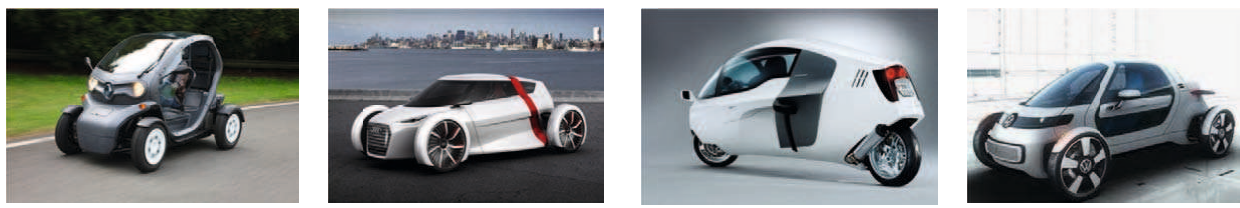

Abbildung 51: Existierende und angekündigte Kleinst-EV (als Kategorie in dieser Studie 3-Wheeler genannt), welche zwei bis vier Räder haben können und unter heutigem Recht zulassungsrechtlich als Motorrad oder Kleinmotorwagen gelten (Bilder: Renault, Audi, Monotracer, Volkswagen).

Bei der Kombination von MIV und ÖV spielt die Reichweitenbegrenzung von EV keine Rolle mehr, was den vermehrten Einsatz namentlich von Kleinst-EV überhaupt erst in grösserem Ausmass erlaubt. Anhand der Daten des Mikrozensus Mobilität und Verkehr 2010 (BFS/ARE 2012) wird nachfolgend abgeschätzt, in 
welcher Grössenordnung dieses Veränderungspotenzial liegen könnte. Abbildung 52 zeigt die Verteilung der 2010 zurückgelegten Etappendistanzen nach Verkehrsmittel.

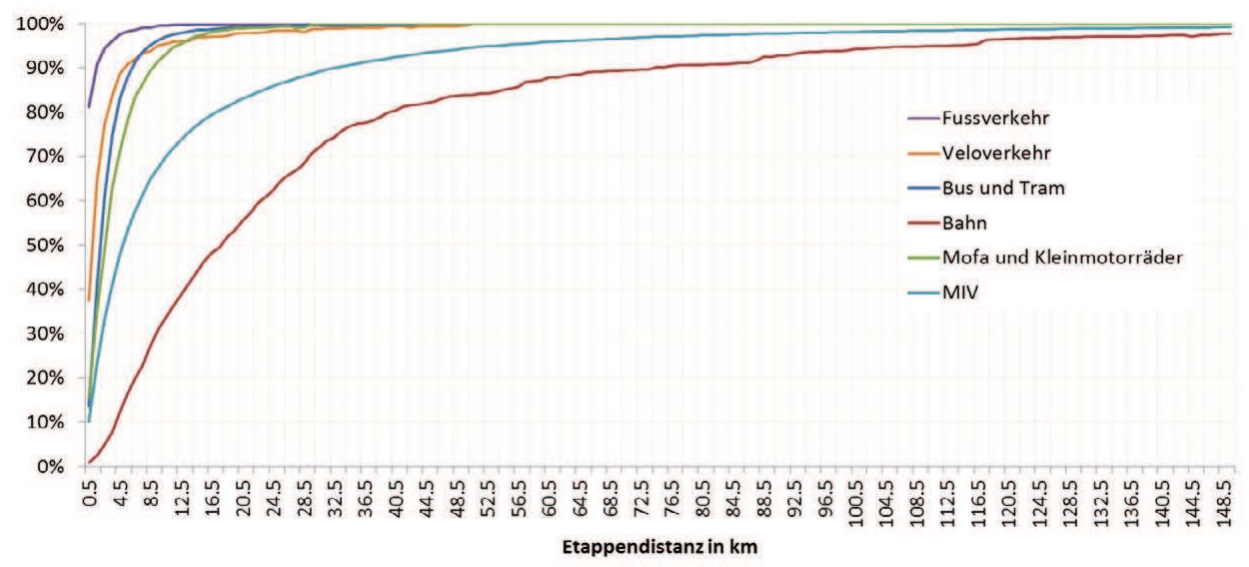

Abbildung 52: Kumulative Verteilung der Etappendistanzen nach Verkehrsmittel. Datenquelle: Mikrozensus Mobilität und Verkehr 2010 von BFS und ARE.

Aus Abbildung 52 lassen sich die Mediane (die Hälfte der Fahrten ist kürzer als der Median) ablesen, wie in Tabelle 28 zusammengefasst.

Fuss und Velo Bus und Tram Bahn Mofa/Klein MR MIV

ca. $0,5 \mathrm{~km}$

ca. $2,5 \mathrm{~km}$

ca. $18,5 \mathrm{~km}$

ca. $3,5 \mathrm{~km}$

ca. $5,5 \mathrm{~km}$

Tabelle 28: $\quad$ Mediane der Etappendistanzen nach Verkehrsmittel.

Interessant ist der grosse Unterschied zwischen Medianwert der Bahnfahrten und Medianwert der MIV-Fahrten. Trotz sehr grosser Zahl kurzer MIV-Fahrten ergeben sich jedoch auch sehr lange MIV-Fahrten, und auch diese in relativ grosser Zahl, wie sich aus Abbildung 53 ableiten lässt. Zwischen den verschie- 
denen Klassen der Etappendistanzen ergeben sich aus dem Mikrozensus 2010 gemäss Abbildung 53 stark unterschiedliche Modalsplits.

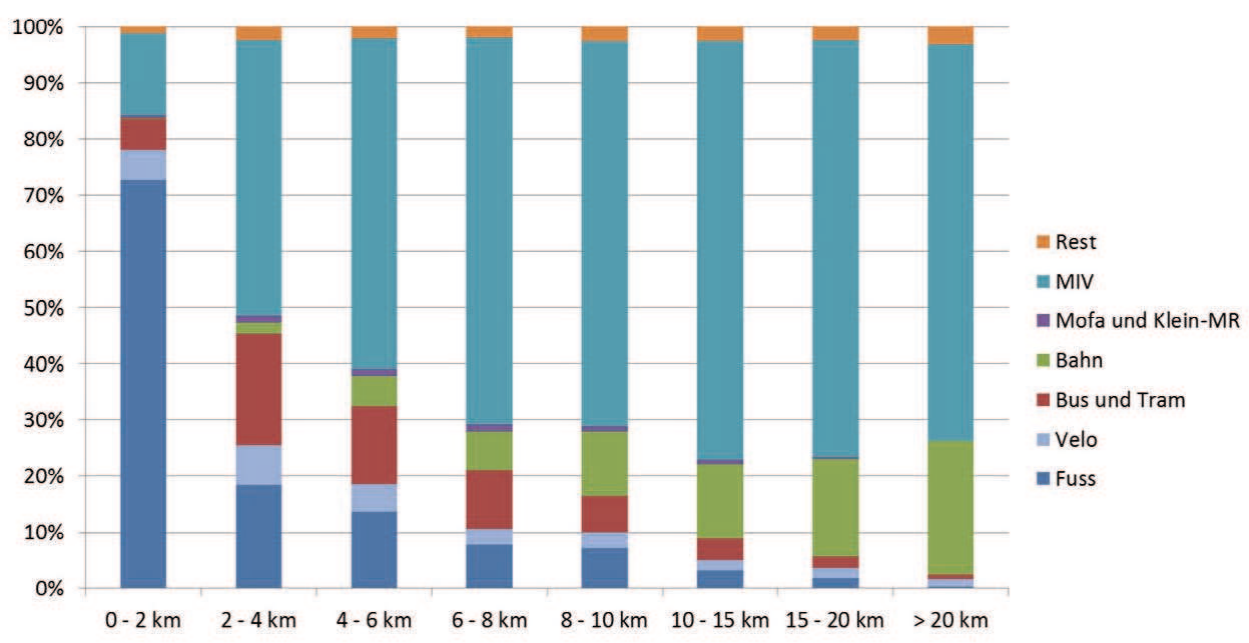

Abbildung 53: Modalsplit der Anzahl Etappen, nach Distanzklassen.

Der MIV-Anteil ist in den Distanzklassen zwischen 10 und 20 Kilometer mit rund 75 Prozent am höchsten. In der Distanzklasse von mehr als 20 Kilometer beträgt er immer noch über 70 Prozent. Und trotz des tiefen MIV-Medianwertes von 5,5 Kilometer, gemessen an der Anzahl Etappen, ist der Modalsplit des MIV auch bei Fahrten über 20 Kilometer Länge zirka dreimal so hoch wie jener der Bahn. In diesem Distanzbereich liegt das Potenzial des COM-Szenarios.

Auch die vielen kurzen MIV-Fahrten bis zirka 5,5 Kilometer sind für eine Substitution von MIV-Fahrleistung interessant. In diesem Distanzbereich zeigt sich Potenzial für eine Verschiebung zum Langsamverkehr (Velos und E-Bikes), aber auch zum Segment der Kleinst-EV.

Für die Verlagerung der MIV-Fahrtleistung hin zu Kleinst-EV werden deshalb folgende zwei Fälle betrachtet:

1. Ein Anteil der kurzen MIV-Fahrten $(<5 \mathrm{~km}$ ) wird durch Kleinst-EV ersetzt; auch wenn der Energiebedarf dadurch abnimmt, reduziert dies nicht die gefahrenen Kilometer. Wenn in einer Fahrleistungsstatistik aber nach Perso- 
nenwagen einerseits und Motorrädern (inkl. Leichtmotorwagen) anderseits unterschieden wird, ergibt sich eine Verschiebung der Fahrleistung weg von den Personenwagen.

2. Ein Anteil der langen MIV-Fahrten ( $>20 \mathrm{~km}$ ) wird durch die Bahn ersetzt. Die Etappen zum und vom Bahnhof erfolgen mittels Kleinst-EV.

Abbildung 54 zeigt illustrativ die aus (1) und (2) zu erwartenden Änderungen der Etappendistanzen je Verkehrsmittel.

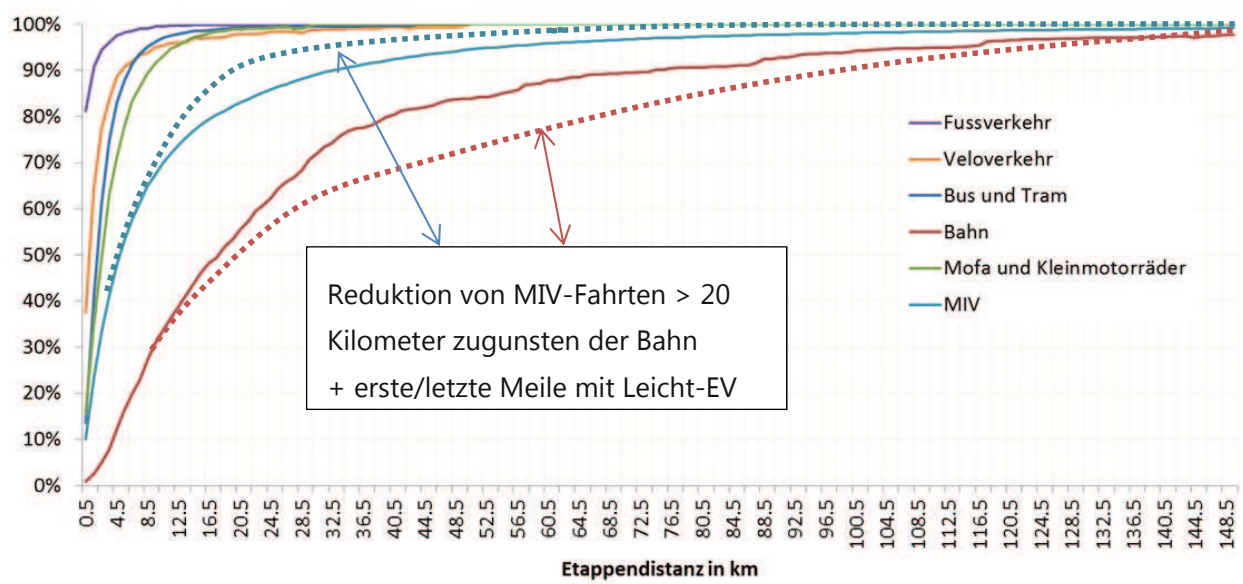

Abbildung 54: Zu erwartende Verschiebungen der kumulativen Häufigkeitsverteilung der Etappendistanzen (illustrative Darstellung).

Die mit dem Mikrozensus erhobene MIV-Fahrleistung (Stichprobenumfang zirka 63000 Personen ab sechs Jahren) beträgt über alle Fahrtzwecke rund 1,1 Millionen Fahrzeugkilometer. Davon fallen 10 Prozent auf Fahrten unter 5 Kilometer und 65 Prozent auf Fahrten von mehr als 20 Kilometern.

Vom theoretischen Verlagerungspotenzial dieser Segmente im Sinne von (1) oder (2) (100 Prozent) kann in der Praxis nur ein Teil realisiert werden. Eine Verminderung ergibt sich insbesondere aus folgenden Gründen: Zwangsbenützter MIV wegen gleichzeitigem Gütertransport, Serviceaufgaben, diverse Mobilitätsbehinderungen (Erfahrungen sprechen von zirka 20 bis 25 Prozent), Reisezeitverhältnisse (geschätzt werden 30 bis 50 Prozent), fehlende geeignete $P+R-A b-$ 
stellplätze. Das realisierbare Verlagerungspotenzial der MIV-Fahrleistung durch den vermehrten Einsatz von 3- oder leichten Kleinst-EV dürfte zwischen 4 und 10 Prozent der Distanzbereiche bis 5 und ab 20 Kilometer liegen.

Im COM-Szenario wird von einem Anteil der Kleinst-EV an der Gesamtfahrleistung ausgegangen, der bis 2035 auf 1,5 Prozent ansteigt (1,1 von 71,4 Milliarden Fahrzeugkilometer) und bis 2050 6,8 Prozent erreicht (5,1 von 75,1 Milliarden Fahrzeugkilometer). Gründe, die eine Reduktion der MIV-Fahrleistung per zirka 2025 bis 2030 um diese Werte als realistisch erscheinen lassen, sind:

3. Zunehmende Auslastung des Strassennetzes und verschärfte Stausituationen; während die mittlere Fahrzeit für längere MIV-Etappen dadurch geringfügig ansteigt, leiden die Planbarkeit und Zuverlässigkeit des Verkehrsmittels Auto überproportional.

4. Allgemeiner Wertewandel im Bereich Mobilität aufgrund abnehmender Verfügbarkeit sowie Verteuerung fossiler Treibstoffe und zunehmende geopolitische Abhängigkeiten und/oder veränderte Wahrnehmung und Gewichtung der Folgen des Klimawandels.

5. Neue Angebote (Kleinst-EV-Leasing durch die SBB) sowie neue Geschäftsmodelle (EVU vermieten EV, kombiniert mit einem Ökostrom-Angebot und/ oder Energy Contracting für das Eigenheim, und können über die Kontrolle des Home Charge Device von koordiniertem Smart Charge profitieren sowie von der Kommerzialisierung der Reserveleistung der zu einer virtuellen Einheit zusammengefassten EV).

6. Präferenz der Konsumenten für teilautarke Lösungen mit gewissen eigenen Handlungsmöglichkeiten: Kombination von EV mit dezentraler Fotovoltaik, und Einbezug des EV-Ladeverhaltens und der EV-Batterie in das SmartHome-Energiemanagement.

7. Weiterhin Zunahme der Attraktivität des ÖV, insbesondere auf der Schiene (weitere Taktverdichtung und erleichterter Zugang dank elektronischem Ticketing).

8. Entstehen von Kernstädten mit stark reduziertem Zugang für MIV, was dazu führt, dass nahezu alle Bevölkerungsschichten mindestens sporadisch den ÖV nutzen und damit die Eintrittsbarrieren für vermehrte ÖV-Nutzung sehr gering sind. 


\section{A4 Detaillierte Grundlagen zu Kapitel 2 (Marktpenetration von Elektromo- bilen)}

Die nachstehenden Tabellen zeigen die Entwicklung der Energieeffizienz und der regulatorischen $\mathrm{g} \mathrm{CO}_{2} / \mathrm{km}$-Mittelwerte.

\begin{tabular}{|c|c|c|c|c|c|c|c|c|c|}
\hline & 2015 & 2016 & 2017 & 2018 & 2019 & 2020 & 2025 & 2030 & 2035 \\
\hline $\mathrm{g} \mathrm{CO}_{2} / \mathrm{km}$ - ICE (inkI. HEV) & 129.6 & 126.2 & 122.9 & 119.6 & 116.5 & 113.4 & 105.8 & 98.7 & 92.0 \\
\hline $\mathrm{g} \mathrm{CU}_{2} / \mathrm{km}$ - HHEV & 50 & 40 & 40 & 40 & 40 & 40 & 30 & 25 & 20 \\
\hline $\mathrm{g} \mathrm{CO}_{2} / \mathrm{km}-\mathrm{EV}$ & 0 & 0 & 0 & 0 & 0 & 0 & 0 & 0 & 0 \\
\hline $\mathrm{kWh} / 100 \mathrm{~km}$ - PHEV & 18.4 & 18.1 & 17.9 & 17.7 & 17.4 & 17.2 & 17.3 & 16.8 & 16.3 \\
\hline kWh/100km - EV & 25.7 & 24.9 & 24.2 & 23.5 & 22.7 & 22.1 & 22.4 & 21.0 & 19.6 \\
\hline Anteil an den Neuzulassungen - ICE & $99.2 \%$ & $99.2 \%$ & $99.2 \%$ & $99.2 \%$ & $99.2 \%$ & $99.1 \%$ & $96.4 \%$ & $88.7 \%$ & $75.5 \%$ \\
\hline Anteil an den Neuzulassungen - PHEV & $0.4 \%$ & $0.4 \%$ & $0.4 \%$ & $0.4 \%$ & $0.4 \%$ & $0.4 \%$ & $1.8 \%$ & $5.6 \%$ & $12.3 \%$ \\
\hline Anteil an den Neuzulassungen - EV & $0.4 \%$ & $0.4 \%$ & $0.4 \%$ & $0.4 \%$ & $0.4 \%$ & $0.4 \%$ & $1.8 \%$ & $5.6 \%$ & $12.3 \%$ \\
\hline \multirow[t]{2}{*}{$\mathrm{g} \mathrm{CO}_{2} / \mathrm{fzkm}$ - Mittelwert } & 128.8 & 125.3 & 122.0 & 118.8 & 115.7 & 112.6 & 102.6 & 88.9 & 71.9 \\
\hline & 2015 & 2016 & 2017 & 2018 & 2019 & 2020 & 2025 & 2030 & 2035 \\
\hline $\mathrm{g} \mathrm{CO}_{2} / \mathrm{km}$ - ICE (inkI. HEV) & 129.6 & 126.2 & 122.9 & 119.6 & 116.5 & 113.4 & 106.9 & 102.5 & 98.3 \\
\hline $\mathrm{g} \cup_{2} / \mathrm{km}$ - HHEV & 50 & 40 & 40 & 40 & 40 & 40 & 30 & 25 & 20 \\
\hline $\mathrm{g} \mathrm{CO}_{2} / \mathrm{km}-\mathrm{EV}$ & 0 & 0 & 0 & 0 & 0 & 0 & 0 & 0 & 0 \\
\hline $\mathrm{kWh} / 100 \mathrm{~km}$ - PHEV & 16.0 & 15.8 & 15.5 & 15.3 & 15.1 & 14.9 & 15.0 & 14.6 & 14.1 \\
\hline kWh/100km - EV & 22.3 & 21.7 & 21.0 & 20.4 & 19.8 & 19.2 & 19.5 & 18.2 & 17.0 \\
\hline Anteil an den Neuzulassungen - ICE & $99.2 \%$ & $99.1 \%$ & $98.9 \%$ & $98.6 \%$ & $98.2 \%$ & $97.8 \%$ & $90.2 \%$ & $73.5 \%$ & $48.8 \%$ \\
\hline Anteil an den Neuzulassungen - PHEV & $0.4 \%$ & $0.5 \%$ & $0.5 \%$ & $0.7 \%$ & $0.8 \%$ & $1.0 \%$ & $3.7 \%$ & $8.3 \%$ & $12.8 \%$ \\
\hline Anteil an den Neuzulassungen - EV & $0.4 \%$ & $0.5 \%$ & $0.6 \%$ & $0.8 \%$ & $1.0 \%$ & $1.3 \%$ & $6.1 \%$ & $18.2 \%$ & $38.4 \%$ \\
\hline \multirow[t]{2}{*}{$\mathrm{g} \mathrm{CO}_{2} / \mathrm{fzkm}$ - Mittelwert } & 128.8 & 125.2 & 121.7 & 118.2 & 114.7 & 111.3 & 97.5 & 77.4 & 50.5 \\
\hline & 2015 & 2016 & 2017 & 2018 & 2019 & 2020 & 2025 & 2030 & 2035 \\
\hline $\mathrm{g} \mathrm{CO}_{2} / \mathrm{km}$ - ICE (inkl. HEV) & 129.6 & 123.9 & 118.5 & 113.3 & 108.3 & 103.6 & 94.4 & 90.6 & 86.8 \\
\hline $\mathrm{g} \mathrm{CU}_{2} / \mathrm{km}$ - HHEV & 50 & 40 & 40 & 40 & 40 & 40 & 30 & 25 & 20 \\
\hline $\mathrm{g} \mathrm{CO}_{2} / \mathrm{km}-\mathrm{EV}$ & 0 & 0 & 0 & 0 & 0 & 0 & 0 & 0 & 0 \\
\hline $\mathrm{kWh} / 100 \mathrm{~km}$ - PHEV & 15.2 & 15.0 & 14.8 & 14.6 & 14.4 & 14.2 & 14.3 & 13.8 & 13.4 \\
\hline kWh/100km - EV & 21.2 & 20.6 & 19.9 & 19.3 & 18.8 & 18.2 & 18.5 & 17.3 & 16.1 \\
\hline Anteil an den Neuzulassungen - ICE & $99.2 \%$ & $98.9 \%$ & $98.5 \%$ & $98.2 \%$ & $97.8 \%$ & $97.2 \%$ & $86.1 \%$ & $59.8 \%$ & $37.8 \%$ \\
\hline Anteil an den Neuzulassungen - PHEV & $0.4 \%$ & $0.5 \%$ & $0.7 \%$ & $0.8 \%$ & $0.9 \%$ & $1.0 \%$ & $3.5 \%$ & $5.0 \%$ & $0.0 \%$ \\
\hline Anteil an den Neuzulassungen - EV & $0.4 \%$ & $0.6 \%$ & $0.8 \%$ & $1.0 \%$ & $1.3 \%$ & $1.7 \%$ & $10.4 \%$ & $35.1 \%$ & $62.2 \%$ \\
\hline $\mathrm{g} \mathrm{CO}_{2} / \mathrm{fzkm}$ - Mittelwert & 128.8 & 122.8 & 117.0 & 111.6 & 106.3 & 101.1 & 82.4 & 55.4 & 32.8 \\
\hline
\end{tabular}

Tabelle 29: $\quad g \mathrm{CO}_{2} / \mathrm{km}$-Mittelwerte der PKW-Neuzulassungen, getrennt für ICE/HEV, PHEV und EV. Es handelt sich hier um die Normwerte gemäss NEFZ-Typprüfzyklus, die $\mathrm{g} \mathrm{CO}_{2} / \mathrm{km}$-Werte für PHEV und EV sind regulatorisch vorgegebene Werte im Rahmen des Vollzugs zur $130 \mathrm{~g} \mathrm{CO}_{2} / \mathrm{km}$-Regulierung. Für die Lebenszyklusberechnungen im nächsten Kapitel werden hingegen reale Energieverbrauchswerte sowie andere Strommixe unterlegt. Oben: BAU-, Mitte: EFF-, Unten: COM-Szenario. 


\begin{tabular}{|c|c|c|c|c|c|c|c|c|c|}
\hline & 2015 & 2016 & 2017 & 2018 & 2019 & 2020 & 2025 & 2030 & 2035 \\
\hline \multicolumn{10}{|l|}{$\mathrm{CO}_{2}$-Emissionen [g/fzkm] } \\
\hline Neuzulassungen: BAU & 128.8 & 125.3 & 122.0 & 118.8 & 115.7 & 112.6 & 102.6 & 88.9 & 71.9 \\
\hline Fahrende Fotte: BAU & 174.8 & 169.6 & 164.4 & 159.2 & 154.1 & 149.1 & 129.6 & 114.0 & 98.2 \\
\hline Fahrleistung der PKW [Mio. fzkm/a] & $63^{\prime} 414$ & $63 ' 955$ & $64 ' 495$ & $65^{\prime} 036$ & $65^{\prime} 577$ & $66^{\prime} 117$ & $67^{\prime} 749$ & $69^{\prime} 360$ & $71^{\prime} 363$ \\
\hline davon ICE: BAU & $63^{\prime} 316$ & $63^{\prime} 818$ & $64^{\prime} 319$ & $64 ' 819$ & $65^{\prime} 319$ & $65^{\prime} 818$ & $666^{\prime} 866$ & $66^{\prime} 501$ & $63 ' 812$ \\
\hline davon PHEV: BAU & 47 & 66 & 86 & 106 & 127 & 147 & 439 & $1 ' 427$ & 3'773 \\
\hline davon EV: BAU & 47 & 66 & 86 & 106 & 127 & 147 & 439 & $1^{\prime} 427$ & $3^{\prime} 773$ \\
\hline \multicolumn{10}{|l|}{ Energie [PJ] } \\
\hline $\mathrm{B} / \mathrm{D} / \mathrm{G}, \mathrm{BAU}$ & 150 & 147 & 144 & 140 & 137 & 134 & 119 & 107 & 95 \\
\hline E, BAU & 0.07 & 0.10 & 0.13 & 0.16 & 0.18 & 0.2 & 0.6 & 1.9 & 4.9 \\
\hline \multicolumn{10}{|l|}{$\mathrm{CO}_{2}$-Emissionen [Mio t/a] } \\
\hline $\mathrm{B} / \mathrm{D} / \mathrm{G}, \mathrm{BAU}$ & 11.1 & 10.8 & 10.6 & 10.4 & 10.1 & 9.9 & 8.8 & 7.9 & 7.0 \\
\hline \multicolumn{10}{|l|}{ Treibstoffabsatz BAU } \\
\hline $\mathrm{B}+\mathrm{G}[\mathrm{PJ}]$ & 94 & 90 & 87 & 83 & 79 & 76 & 65 & 58 & 51 \\
\hline $\mathrm{D}[\mathrm{PJ}]$ & 56 & 57 & 57 & 58 & 58 & 58 & 54 & 49 & 44 \\
\hline $\mathrm{B}+\mathrm{G}[\mathrm{Mio} \mathrm{kg}]$ & $2^{\prime} 215$ & $2^{\prime} 126$ & $2^{\prime} 037$ & 1'949 & 1'862 & $1^{\prime} 777$ & 1'519 & 1'364 & 1'192 \\
\hline $\mathrm{D}$ [Mio kg] & 1'312 & 1'325 & 1'336 & $1 ' 345$ & $1 ' 353$ & 1'359 & 1'273 & $1 ' 149$ & 1'037 \\
\hline B+G [Mio. Liter] & $2^{\prime} 974$ & $2^{\prime} 854$ & $2 ' 734$ & $2^{\prime} 616$ & $2^{\prime} 500$ & $2^{\prime} 386$ & $2^{\prime} 038$ & 1'831 & $1 ' 600$ \\
\hline D [Mio. Liter] & 1'582 & 1'598 & $1^{\prime} 612$ & $1^{\prime} 623$ & $1^{\prime} 632$ & 1'639 & 1'535 & 1'386 & 1'251 \\
\hline
\end{tabular}

Tabelle 30: $\quad \mathrm{CO}_{2}$-Emissionen und Fahrleistungen, resultierender Energieverbrauch und Treibstoffabsätze für das BAU-Szenario

\begin{tabular}{|c|c|c|c|c|c|c|c|c|c|}
\hline & 2015 & 2016 & 2017 & 2018 & 2019 & 2020 & 2025 & 2030 & 2035 \\
\hline \multicolumn{10}{|l|}{$\mathrm{CO}_{2}$-Emissionen [g/fzkm] } \\
\hline Neuzulassungen: & 128.8 & 125.2 & 121.7 & 118.2 & 114.7 & 111.3 & 97.5 & 77.4 & 50.5 \\
\hline Fahrende Hotte: घ下 & 174.8 & 169.6 & 164.3 & 159.1 & 153.9 & 148.8 & 128.1 & 109.1 & 86.4 \\
\hline Fahrleistung der PKW [Mio. fzkm/a] & $63^{\prime} 414$ & $63 ' 955$ & $64 ' 495$ & $65^{\prime} 036$ & $65^{\prime} 577$ & $66^{\prime} 117$ & $67^{\prime} 749$ & $69 ' 360$ & $71^{\prime} 363$ \\
\hline davon ICE: E干 & $63^{\prime} 318$ & $63^{\prime} 812$ & $64^{\prime} 297$ & $64^{\prime} 768$ & $65^{\prime} 218$ & $65^{\prime} 649$ & $65^{\prime} 871$ & $62 ' 857$ & $54^{\prime} 713$ \\
\hline davon PHEV: 由干 & 41 & 63 & 90 & 122 & 163 & 211 & 761 & $2^{\prime} 274$ & 4'924 \\
\hline davon EV: E干 & 51 & 75 & 104 & 141 & 191 & 253 & 1'112 & $4^{\prime} 224$ & $11 ' 721$ \\
\hline \multicolumn{10}{|l|}{ Energie [PJ] } \\
\hline $\mathrm{B} / \mathrm{D} / \mathrm{G}$, 旦干 & 150 & 147 & 144 & 140 & 137 & 133 & 118 & 103 & 84 \\
\hline E, E开 & 0.06 & 0.09 & 0.13 & 0.17 & 0.22 & 0.3 & 1.2 & 4.0 & 9.7 \\
\hline \multicolumn{10}{|l|}{$\mathrm{CO}_{2}$-Emissionen [Mio t/a] } \\
\hline $\mathrm{B} / \mathrm{D} / \mathrm{G}, \mathrm{ET}$ & 11.1 & 10.8 & 10.6 & 10.3 & 10.1 & 9.8 & 8.7 & 7.6 & 6.2 \\
\hline \multicolumn{10}{|l|}{ Treibstoffabsatz 日一 } \\
\hline$B+G[P J]$ & 94 & 90 & 87 & 83 & 79 & 75 & 64 & 56 & 45 \\
\hline$D[P J]$ & 56 & 57 & 57 & 58 & 58 & 58 & 54 & 47 & 39 \\
\hline $\mathrm{B}+\mathrm{G}[\mathrm{Mio} \mathrm{kg}]$ & $2^{\prime} 215$ & $2^{\prime} 126$ & $2^{\prime} 036$ & $1 ' 948$ & $1^{\prime} 860$ & $1 ' 774$ & 1'502 & $1 ' 307$ & $1 ' 049$ \\
\hline $\mathrm{D}$ [Mio kg] & 1'312 & 1'325 & 1'336 & $1 ' 344$ & $1 ' 351$ & 1'356 & 1'259 & 1'101 & 912 \\
\hline $\mathrm{B}+\mathrm{G}$ [Mio. Liter] & $2^{\prime} 974$ & $2^{\prime} 853$ & $2^{\prime} 734$ & $2^{\prime} 614$ & $2^{\prime} 497$ & $2^{\prime} 381$ & $2^{\prime} 016$ & $1^{\prime} 754$ & $1 ' 408$ \\
\hline D [Mio. Liter] & 1'582 & 1'598 & 1'611 & $1^{\prime} 622$ & $1^{\prime} 630$ & $1^{\prime} 636$ & 1'518 & $1 ' 328$ & $1 ' 100$ \\
\hline
\end{tabular}

Tabelle 31: $\quad \mathrm{CO}_{2}$-Emissionen und Fahrleistungen, resultierender Energieverbrauch und Treibstoffabsätze für das EFF-Szenario. 


\begin{tabular}{|c|c|c|c|c|c|c|c|c|c|}
\hline & 2015 & 2016 & 2017 & 2018 & 2019 & 2020 & 2025 & 2030 & 2035 \\
\hline \multicolumn{10}{|l|}{$\mathrm{CO}_{2}$-Emissionen [g/fzkm] } \\
\hline Neuzulassungen: COM & 128.8 & 122.8 & 117.0 & 111.6 & 106.3 & 101.1 & 82.4 & 55.4 & 32.8 \\
\hline Fahrende Fotte: COM & 174.8 & 169.4 & 163.8 & 157.9 & 152.0 & 145.9 & 119.2 & 93.8 & 67.6 \\
\hline Fahrleistung der PKW [Mio. fzkm/a] & $63 ' 414$ & $63^{\prime} 955$ & $64 ' 495$ & $65^{\prime} 036$ & $65^{\prime} 577$ & $66^{\prime} 117$ & $67 ' 749$ & $69^{\prime} 360$ & $71 ' 363$ \\
\hline davon ICE COM & $63 ' 317$ & $63 ' 804$ & $64^{\prime} 273$ & $64^{\prime} 725$ & $65^{\prime} 160$ & $65^{\prime} 563$ & $64^{\prime} 993$ & $59^{\prime} 466$ & $48 ' 699$ \\
\hline davon PHEV: COM & 41 & 67 & 99 & 136 & 179 & 230 & 852 & 1'962 & 2'228 \\
\hline davon EV: COM & 51 & 80 & 119 & 169 & 233 & 319 & 1'899 & 7'928 & $20 ' 431$ \\
\hline \multicolumn{10}{|l|}{ Energie [PJ] } \\
\hline B/D/G, COM & 150 & 147 & 143 & 139 & 135 & 131 & 110 & 88 & 65 \\
\hline E, COM & 0.06 & 0.09 & 0.14 & 0.19 & 0.25 & 0.3 & 1.7 & 5.9 & 12.9 \\
\hline \multicolumn{10}{|l|}{$\mathrm{CO}_{2}$-Emissionen [Mio t/a] } \\
\hline B/D/G, COM & 11.1 & 10.8 & 10.6 & 10.3 & 10.0 & 9.6 & 8.1 & 6.5 & 4.8 \\
\hline \multicolumn{10}{|l|}{ Treibstoffabsatz COM } \\
\hline$B+G[P J]$ & 94 & 90 & 86 & 82 & 78 & 74 & 59 & 48 & 35 \\
\hline $\mathrm{D}[\mathrm{PJ}]$ & 56 & 57 & 57 & 57 & 57 & 57 & 50 & 40 & 31 \\
\hline $\mathrm{B}+\mathrm{G}$ [Mio kg] & $2^{\prime} 215$ & $2 ' 124$ & $2^{\prime} 030$ & 1'933 & 1'836 & $1 ' 739$ & 1'397 & $1 ' 123$ & 821 \\
\hline $\mathrm{D}$ [Mio kg] & 1'312 & 1'324 & 1'331 & 1'335 & 1'334 & 1'329 & 1'171 & 946 & 714 \\
\hline B+G [Mio. Liter] & 2'974 & $2^{\prime} 851$ & $2^{\prime} 724$ & 2'595 & $2^{\prime} 465$ & $2^{\prime} 334$ & $1 ' 876$ & 1'507 & 1'102 \\
\hline D [Mio. Liter] & 1'582 & 1'597 & 1'606 & 1'610 & $1^{\prime} 609$ & $1 ' 603$ & $1^{\prime} 413$ & 1'141 & 861 \\
\hline
\end{tabular}

Tabelle 32: $\quad \mathrm{CO}_{2}$-Emissionen und Fahrleistungen, resultierender Energieverbrauch und Treibstoffabsätze für das COM-Szenario. 


\section{A5 Detaillierte Grundlagen zu Kapitel 5 (Lebenszyklusanalyse)}

\begin{tabular}{|c|c|c|c|c|c|c|c|c|}
\hline & & \multicolumn{7}{|c|}{ Vehicle Parameters } \\
\hline & & $\begin{array}{c}\text { Vehicle total } \\
\text { mass with } \\
\text { load and } \\
\text { driver }\end{array}$ & $\begin{array}{c}\text { Vehicle } \\
\text { glider mass }\end{array}$ & $\begin{array}{c}\text { Drivetrain } \\
\text { mass ICE } \\
\text { w/o battery }\end{array}$ & $\begin{array}{l}\text { Drivetrain } \\
\text { mass } \\
\text { electric w/o } \\
\text { battery }\end{array}$ & Front area & $\begin{array}{l}\text { Air drag } \\
\text { coefficient }\end{array}$ & $\begin{array}{l}\text { Rolling } \\
\text { resistance } \\
\text { coefficient }\end{array}$ \\
\hline Vehicle & Drivetrain & $\mathrm{kg}$ & $\mathrm{kg}$ & $\mathrm{kg}$ & $\mathrm{kg}$ & $\mathrm{A}[\mathrm{m} 2]$ & C_w $[-]$ & c_r $r[-]$ \\
\hline \multirow[t]{3}{*}{ Fullsize "SUV" 2012} & ICE & $2 ' 100$ & $1 ' 850$ & 250 & - & 2.78 & 0.35 & 0.012 \\
\hline & PHEV & 2'300 & 1'850 & 150 & 150 & 2.78 & 0.35 & 0.012 \\
\hline & BEV & 2,400 & $1 ' 850$ & - & 150 & 2.78 & 0.35 & 0.012 \\
\hline \multirow[t]{3}{*}{ Compact "Golf" 2012} & ICE & $1 ' 400$ & $1 ' 250$ & 150 & - & 2.22 & 0.31 & 0.011 \\
\hline & PHEV & 1'550 & $1 ' 250$ & 100 & 100 & 2.22 & 0.31 & 0.011 \\
\hline & BEV & $1^{\prime} 650$ & $1 ' 250$ & - & 100 & 2.22 & 0.31 & 0.011 \\
\hline \multirow[t]{3}{*}{ Micro "Smart" 2012} & ICE & $1 ' 000$ & 850 & 150 & - & 2.00 & 0.35 & 0.011 \\
\hline & PHEV & 1'130 & 850 & 100 & 100 & 2.00 & 0.35 & 0.011 \\
\hline & BEV & $1^{\prime} 150$ & 850 & - & 100 & 2.00 & 0.35 & 0.011 \\
\hline 3-Wheeler "RAKe" 2012 & BEV & 480 & 350 & - & 50 & 1.44 & 0.25 & 0.010 \\
\hline \multirow[t]{3}{*}{ Fullsize "SUV" 2020} & ICE & 1'932 & 1707 & 225 & - & 2.78 & 0.33 & 0.011 \\
\hline & PHEV & 2'127 & $1 ' 707$ & 135 & 135 & 2.78 & 0.33 & 0.011 \\
\hline & BEV & 2'242 & $1 ' 707$ & - & 135 & 2.78 & 0.33 & 0.011 \\
\hline \multirow[t]{3}{*}{ Compact "Golf" 2020} & ICE & 1'288 & 1'153 & 135 & - & 2.22 & 0.29 & 0.010 \\
\hline & PHEV & $1 ' 433$ & 1'153 & 90 & 90 & 2.22 & 0.29 & 0.010 \\
\hline & BEV & 1'543 & 1'153 & - & 90 & 2.22 & 0.29 & 0.010 \\
\hline \multirow[t]{3}{*}{ Micro "Smart" 2020} & ICE & 919 & 784 & 135 & - & 2.00 & 0.33 & 0.010 \\
\hline & PHEV & 1'044 & 784 & 90 & 90 & 2.00 & 0.33 & 0.010 \\
\hline & BEV & $1^{\prime} 074$ & 784 & - & 90 & 2.00 & 0.33 & 0.010 \\
\hline 3-Wheeler "RAKe" 2020 & BEV & 448 & 323 & - & 45 & 1.44 & 0.23 & 0.009 \\
\hline \multirow[t]{3}{*}{ Fullsize "SUV" 2035} & ICE & 1786 & $1 ' 583$ & 203 & - & 2.78 & 0.31 & 0.010 \\
\hline & PHEV & 1'976 & 1'583 & 122 & 122 & 2.78 & 0.31 & 0.010 \\
\hline & BEV & 2'105 & 1'583 & - & 122 & 2.78 & 0.31 & 0.010 \\
\hline \multirow[t]{3}{*}{ Compact "Golf" 2035} & ICE & 1'191 & 1'070 & 122 & - & 2.22 & 0.27 & 0.009 \\
\hline & PHEV & 1'332 & 1'070 & 81 & 81 & 2.22 & 0.27 & 0.009 \\
\hline & BEV & $1 ' 451$ & $1 ' 070$ & - & 81 & 2.22 & 0.27 & 0.009 \\
\hline \multirow[t]{3}{*}{ Micro "Smart" 2035} & ICE & 849 & 728 & 122 & - & 2.00 & 0.31 & 0.009 \\
\hline & PHEV & 970 & 728 & 81 & 81 & 2.00 & 0.31 & 0.009 \\
\hline & BEV & 1'009 & 728 & - & 81 & 2.00 & 0.31 & 0.009 \\
\hline 3-Wheeler "RAKe" 2035 & BEV & 420 & 300 & - & 41 & 1.44 & 0.21 & 0.008 \\
\hline \multirow[t]{3}{*}{ Fullsize "SUV" 2050} & ICE & 1707 & 1'525 & 182 & - & 2.78 & 0.30 & 0.009 \\
\hline & PHEV & 1'894 & $1 ' 525$ & 109 & 109 & 2.78 & 0.30 & 0.009 \\
\hline & BEV & $2 ' 034$ & 1'525 & - & 109 & 2.78 & 0.30 & 0.009 \\
\hline \multirow[t]{3}{*}{ Compact "Golf" 2050} & ICE & $1 ' 140$ & $1 ' 030$ & 109 & - & 2.22 & 0.26 & 0.008 \\
\hline & PHEV & 1'276 & $1 ' 030$ & 73 & 73 & 2.22 & 0.26 & 0.008 \\
\hline & BEV & $1 ' 403$ & 1'030 & - & 73 & 2.22 & 0.26 & 0.008 \\
\hline \multirow[t]{3}{*}{ Micro "Smart" 2050} & ICE & 810 & 701 & 109 & - & 2.00 & 0.30 & 0.008 \\
\hline & PHEV & 927 & 701 & 73 & 73 & 2.00 & 0.30 & 0.008 \\
\hline & BEV & 974 & 701 & - & 73 & 2.00 & 0.30 & 0.008 \\
\hline 3-Wheeler "RAKe" 2050 & BEV & 405 & 289 & - & 36 & 1.44 & 0.20 & 0.007 \\
\hline
\end{tabular}

Tabelle 33: $\quad$ Schlüsselannahmen für die Fahrzeuge und deren Entwicklungen. 


\begin{tabular}{|c|c|c|c|c|c|c|}
\hline & & \multicolumn{5}{|c|}{ Battery } \\
\hline & & $\begin{array}{c}\text { Battery } \\
\text { mass }\end{array}$ & $\begin{array}{l}\text { Battery } \\
\text { specific } \\
\text { energy } \\
\text { content }\end{array}$ & $\begin{array}{l}\text { Battery } \\
\text { capacity }\end{array}$ & $\begin{array}{c}\text { eta_Battery } \\
\text { in/out }\end{array}$ & $\begin{array}{c}\text { Battery life } \\
\text { assumed }\end{array}$ \\
\hline Vehicle & Drivetrain & $\mathrm{kg}$ & $\mathrm{Wh} / \mathrm{kg}$ & $\mathrm{kWh}$ & $\%$ & $\mathrm{~km}$ \\
\hline \multirow[t]{3}{*}{ Fullsize "SUV" 2012} & ICE & - & - & - & $0 \%$ & - \\
\hline & PHEV & 150 & 100 & 15 & $92 \%$ & $100 ' 000$ \\
\hline & BEV & 400 & 100 & 40 & $92 \%$ & $100 \prime 000$ \\
\hline \multirow[t]{3}{*}{ Compact "Golf" 2012} & ICE & - & - & - & $0 \%$ & - \\
\hline & PHEV & 100 & 100 & 10 & $92 \%$ & $100 ' 000$ \\
\hline & BEV & 300 & 100 & 30 & $92 \%$ & $100 ' 000$ \\
\hline \multirow[t]{3}{*}{ Micro "Smart" 2012} & ICE & - & - & - & $0 \%$ & - \\
\hline & PHEV & 80 & 100 & 8 & $92 \%$ & $100 \prime 000$ \\
\hline & BEV & 200 & 100 & 20 & $92 \%$ & $100 \prime 000$ \\
\hline 3-Wheeler "RAKe" 2012 & BEV & 80 & 100 & 8 & $92 \%$ & $100^{\prime} 000$ \\
\hline \multirow[t]{3}{*}{ Fullsize "SUV" 2020} & ICE & - & - & - & $0 \%$ & - \\
\hline & PHEV & 150 & 154 & 23 & $94 \%$ & $150 \prime 000$ \\
\hline & BEV & 400 & 154 & 62 & $94 \%$ & $150 \prime 000$ \\
\hline \multirow[t]{3}{*}{ Compact "Golf" 2020} & ICE & - & - & - & $0 \%$ & - \\
\hline & PHEV & 100 & 154 & 15 & $94 \%$ & $150 \prime 000$ \\
\hline & BEV & 300 & 154 & 46 & $94 \%$ & $150 \prime 000$ \\
\hline \multirow[t]{3}{*}{ Micro "Smart" 2020} & ICE & - & - & - & $0 \%$ & - \\
\hline & PHEV & 80 & 154 & 12 & $94 \%$ & $150 \prime 000$ \\
\hline & BEV & 200 & 154 & 31 & $94 \%$ & $150 \prime 000$ \\
\hline 3-Wheeler "RAKe" 2020 & BEV & 80 & 154 & 12 & $94 \%$ & $150 \prime 000$ \\
\hline \multirow[t]{3}{*}{ Fullsize "SUV" 2035} & ICE & - & - & - & $0 \%$ & - \\
\hline & PHEV & 150 & 313 & 47 & $95 \%$ & $150 \prime 000$ \\
\hline & BEV & 400 & 313 & 125 & $95 \%$ & $150 \prime 000$ \\
\hline \multirow[t]{3}{*}{ Compact "Golf" 2035} & ICE & - & - & - & $0 \%$ & - \\
\hline & PHEV & 100 & 313 & 31 & $95 \%$ & $150 \prime 000$ \\
\hline & BEV & 300 & 313 & 94 & $95 \%$ & $150 \prime 000$ \\
\hline \multirow[t]{3}{*}{ Micro "Smart" 2035} & ICE & - & - & - & $0 \%$ & - \\
\hline & PHEV & 80 & 313 & 25 & $95 \%$ & $150 \prime 000$ \\
\hline & BEV & 200 & 313 & 63 & $95 \%$ & $150 \prime 000$ \\
\hline 3-Wheeler "RAKe" 2035 & BEV & 80 & 313 & 25 & $95 \%$ & $150 \prime 000$ \\
\hline \multirow[t]{3}{*}{ Fullsize "SUV" 2050} & ICE & - & - & - & $0 \%$ & - \\
\hline & PHEV & 150 & 360 & 54 & $96 \%$ & $150 \prime 000$ \\
\hline & BEV & 400 & 360 & 144 & $96 \%$ & $150^{\prime} 000$ \\
\hline \multirow[t]{3}{*}{ Compact "Golf" 2050} & ICE & - & - & - & $0 \%$ & - \\
\hline & PHEV & 100 & 360 & 36 & $96 \%$ & $150 \prime 000$ \\
\hline & BEV & 300 & 360 & 108 & $96 \%$ & $150 \prime 000$ \\
\hline \multirow[t]{3}{*}{ Micro "Smart" 2050} & ICE & - & - & - & $0 \%$ & - \\
\hline & PHEV & 80 & 360 & 29 & $96 \%$ & $150 \prime 000$ \\
\hline & BEV & 200 & 360 & 72 & $96 \%$ & $150^{\prime} 000$ \\
\hline 3-Wheeler "RAKe" 2050 & BEV & 80 & 360 & 29 & $96 \%$ & $150 \prime 000$ \\
\hline
\end{tabular}

Tabelle 34: $\quad$ Schlüsselannahmen zu den Batterien und deren Entwicklung. Die Batteriemassen werden über die Zeit konstant gehalten, was zu einer erheblichen Verbesserung der elektrischen Reichweite durch die steigende Energiedichte führt. Die Lebensdauer wird ab dem Jahr 2020 als so hoch geschätzt, dass kein Ersatz der Batterien während der Lebensdauer des Fahrzeuges mehr nötig sein wird. 


\begin{tabular}{|c|c|c|c|}
\hline & & \multicolumn{2}{|c|}{$\begin{array}{c}\text { Traction: NEDC energy } \\
\text { demand }\end{array}$} \\
\hline & & $\begin{array}{c}\text { ICE: I/100km } \\
\text { gasoline }\end{array}$ & $\begin{array}{c}\text { EVs and } \\
\text { PHEVs } \\
\text { kWh/100km }\end{array}$ \\
\hline Vehicle & Drivetrain & l/100km & $\mathrm{kWh} / 100 \mathrm{~km}$ \\
\hline \multirow[t]{3}{*}{ Fullsize "SUV" 2012} & ICE & 9.1 & \\
\hline & PHEV & 3.0 & 13.2 \\
\hline & BEV & & 22.2 \\
\hline \multirow[t]{3}{*}{ Compact "Golf" 2012} & ICE & 6.0 & \\
\hline & PHEV & 1.9 & 8.6 \\
\hline & BEV & & 14.5 \\
\hline \multirow[t]{3}{*}{ Micro "Smart" 2012} & ICE & 4.8 & \\
\hline & PHEV & 1.6 & 7.2 \\
\hline & BEV & & 11.8 \\
\hline 3-Wheeler "RAKe" 2012 & BEV & & 5.1 \\
\hline \multirow{3}{*}{ Fullsize "SUV" 2020} & ICE & 7.1 & \\
\hline & PHEV & 1.5 & 14.0 \\
\hline & BEV & & 18.8 \\
\hline \multirow[t]{3}{*}{ Compact "Golf" 2020} & ICE & 4.7 & \\
\hline & PHEV & 1.0 & 9.1 \\
\hline & BEV & & 12.4 \\
\hline \multirow[t]{3}{*}{ Micro "Smart" 2020} & ICE & 3.8 & \\
\hline & PHEV & 0.8 & 7.6 \\
\hline & BEV & & 10.1 \\
\hline 3-Wheeler "RAKe" 2020 & BEV & & 4.3 \\
\hline \multirow[t]{3}{*}{ Fullsize "SUV" 2035} & ICE & 5.7 & \\
\hline & PHEV & 0.7 & 13.5 \\
\hline & BEV & & 16.1 \\
\hline \multirow[t]{3}{*}{ Compact "Golf" 2035} & ICE & 3.8 & \\
\hline & PHEV & 0.5 & 8.9 \\
\hline & BEV & & 10.7 \\
\hline \multirow[t]{3}{*}{ Micro "Smart" 2035} & ICE & 3.1 & \\
\hline & PHEV & 0.4 & 7.5 \\
\hline & BEV & & 8.8 \\
\hline 3-Wheeler "RAKe" 2035 & BEV & & 3.7 \\
\hline \multirow[t]{3}{*}{ Fullsize "SUV" 2050} & ICE & 4.8 & \\
\hline & PHEV & 0.4 & 12.6 \\
\hline & BEV & & 14.2 \\
\hline \multirow[t]{3}{*}{ Compact "Golf" 2050} & ICE & 3.2 & \\
\hline & PHEV & 0.3 & 8.3 \\
\hline & BEV & & 9.5 \\
\hline \multirow[t]{3}{*}{ Micro "Smart" 2050} & ICE & 2.6 & \\
\hline & PHEV & 0.2 & 7.0 \\
\hline & BEV & & 7.8 \\
\hline 3-Wheeler "RAKe" 2050 & BEV & & 3.3 \\
\hline
\end{tabular}

Tabelle 35: $\quad$ Energiebedarf der Fahrzeuge zur Fortbewegung im neuen europäischen Fahrzyklus (NEFZ). 


\begin{tabular}{|c|c|c|c|c|c|}
\hline & & \multicolumn{4}{|c|}{ Motor } \\
\hline & & $\begin{array}{c}\text { eta_ICE } \\
\text { urban }\end{array}$ & $\begin{array}{l}\text { eta_ICE } \\
\text { extraurban }\end{array}$ & $\begin{array}{c}\text { eta_el.Motor } \\
\text { urban }\end{array}$ & $\begin{array}{c}\text { eta_el.Motor } \\
\text { extraurban }\end{array}$ \\
\hline Vehicle & Drivetrain & $\%$ & $\%$ & $\%$ & $\%$ \\
\hline \multirow[t]{3}{*}{ Fullsize "SUV" 2012} & ICE & $17 \%$ & $27 \%$ & $0 \%$ & $0 \%$ \\
\hline & PHEV & $25 \%$ & $28 \%$ & $84 \%$ & $89 \%$ \\
\hline & BEV & $0 \%$ & $0 \%$ & $84 \%$ & $89 \%$ \\
\hline \multirow[t]{3}{*}{ Compact "Golf" 2012} & ICE & $17 \%$ & $27 \%$ & $0 \%$ & $0 \%$ \\
\hline & PHEV & $25 \%$ & $28 \%$ & $84 \%$ & $89 \%$ \\
\hline & BEV & $0 \%$ & $0 \%$ & $84 \%$ & $89 \%$ \\
\hline \multirow[t]{3}{*}{ Micro "Smart" 2012} & ICE & $17 \%$ & $27 \%$ & $0 \%$ & $0 \%$ \\
\hline & PHEV & $25 \%$ & $28 \%$ & $84 \%$ & $89 \%$ \\
\hline & BEV & $0 \%$ & $0 \%$ & $84 \%$ & $89 \%$ \\
\hline 3-Wheeler "RAKe" 2012 & BEV & $0 \%$ & $0 \%$ & $84 \%$ & $89 \%$ \\
\hline \multirow[t]{3}{*}{ Fullsize "SUV" 2020} & ICE & $20 \%$ & $29 \%$ & $0 \%$ & $0 \%$ \\
\hline & PHEV & $28 \%$ & $30 \%$ & $85 \%$ & $90 \%$ \\
\hline & BEV & $0 \%$ & $0 \%$ & $85 \%$ & $90 \%$ \\
\hline \multirow[t]{3}{*}{ Compact "Golf" 2020} & ICE & $20 \%$ & $29 \%$ & $0 \%$ & $0 \%$ \\
\hline & PHEV & $28 \%$ & $30 \%$ & $85 \%$ & $90 \%$ \\
\hline & BEV & $0 \%$ & $0 \%$ & $85 \%$ & $90 \%$ \\
\hline \multirow[t]{3}{*}{ Micro "Smart" 2020} & ICE & $20 \%$ & $29 \%$ & $0 \%$ & $0 \%$ \\
\hline & PHEV & $28 \%$ & $30 \%$ & $85 \%$ & $90 \%$ \\
\hline & BEV & $0 \%$ & $0 \%$ & $85 \%$ & $90 \%$ \\
\hline 3-Wheeler "RAKe" 2020 & BEV & $0 \%$ & $0 \%$ & $85 \%$ & $90 \%$ \\
\hline \multirow[t]{3}{*}{ Fullsize "SUV" 2035} & ICE & $23 \%$ & $31 \%$ & $0 \%$ & $0 \%$ \\
\hline & PHEV & $31 \%$ & $32 \%$ & $86 \%$ & $91 \%$ \\
\hline & BEV & $0 \%$ & $0 \%$ & $86 \%$ & $91 \%$ \\
\hline \multirow[t]{3}{*}{ Compact "Golf" 2035} & ICE & $23 \%$ & $31 \%$ & $0 \%$ & $0 \%$ \\
\hline & PHEV & $31 \%$ & $32 \%$ & $86 \%$ & $91 \%$ \\
\hline & BEV & $0 \%$ & $0 \%$ & $86 \%$ & $91 \%$ \\
\hline \multirow[t]{3}{*}{ Micro "Smart" 2035} & ICE & $23 \%$ & $31 \%$ & $0 \%$ & $0 \%$ \\
\hline & PHEV & $31 \%$ & $32 \%$ & $86 \%$ & $91 \%$ \\
\hline & BEV & $0 \%$ & $0 \%$ & $86 \%$ & $91 \%$ \\
\hline 3-Wheeler "RAKe" 2035 & BEV & $0 \%$ & $0 \%$ & $86 \%$ & $91 \%$ \\
\hline \multirow[t]{3}{*}{ Fullsize "SUV" 2050} & ICE & $26 \%$ & $33 \%$ & $0 \%$ & $0 \%$ \\
\hline & PHEV & $34 \%$ & $34 \%$ & $87 \%$ & $92 \%$ \\
\hline & BEV & $0 \%$ & $0 \%$ & $87 \%$ & $92 \%$ \\
\hline \multirow[t]{3}{*}{ Compact "Golf" 2050} & ICE & $26 \%$ & $33 \%$ & $0 \%$ & $0 \%$ \\
\hline & PHEV & $34 \%$ & $34 \%$ & $87 \%$ & $92 \%$ \\
\hline & BEV & $0 \%$ & $0 \%$ & $87 \%$ & $92 \%$ \\
\hline \multirow[t]{3}{*}{ Micro "Smart" 2050} & ICE & $26 \%$ & $33 \%$ & $0 \%$ & $0 \%$ \\
\hline & PHEV & $34 \%$ & $34 \%$ & $87 \%$ & $92 \%$ \\
\hline & BEV & $0 \%$ & $0 \%$ & $87 \%$ & $92 \%$ \\
\hline 3-Wheeler "RAKe" 2050 & BEV & $0 \%$ & $0 \%$ & $87 \%$ & $92 \%$ \\
\hline \multirow{5}{*}{$\begin{array}{l}\text { Empa Touran Benzin } \\
\text { Empa Avensis Diesel } \\
\text { Mitsubishi iMiev } \\
\text { Empa Prius Benzin } \\
\text { Empa Touran GasHybrid }\end{array}$} & ICE & $21 \%$ & $28 \%$ & $83 \%$ & $88 \%$ \\
\hline & ICE & $26 \%$ & $30 \%$ & $83 \%$ & $88 \%$ \\
\hline & BEV & $0 \%$ & $0 \%$ & $83 \%$ & $88 \%$ \\
\hline & ICE & $30 \%$ & $32 \%$ & $83 \%$ & $88 \%$ \\
\hline & ICE & $30 \%$ & $32 \%$ & $83 \%$ & $88 \%$ \\
\hline
\end{tabular}

Tabelle 36: $\quad$ Annahmen zu den Wirkungsgraden der Antriebe im städtischen und ausserstädtischen Betrieb. 


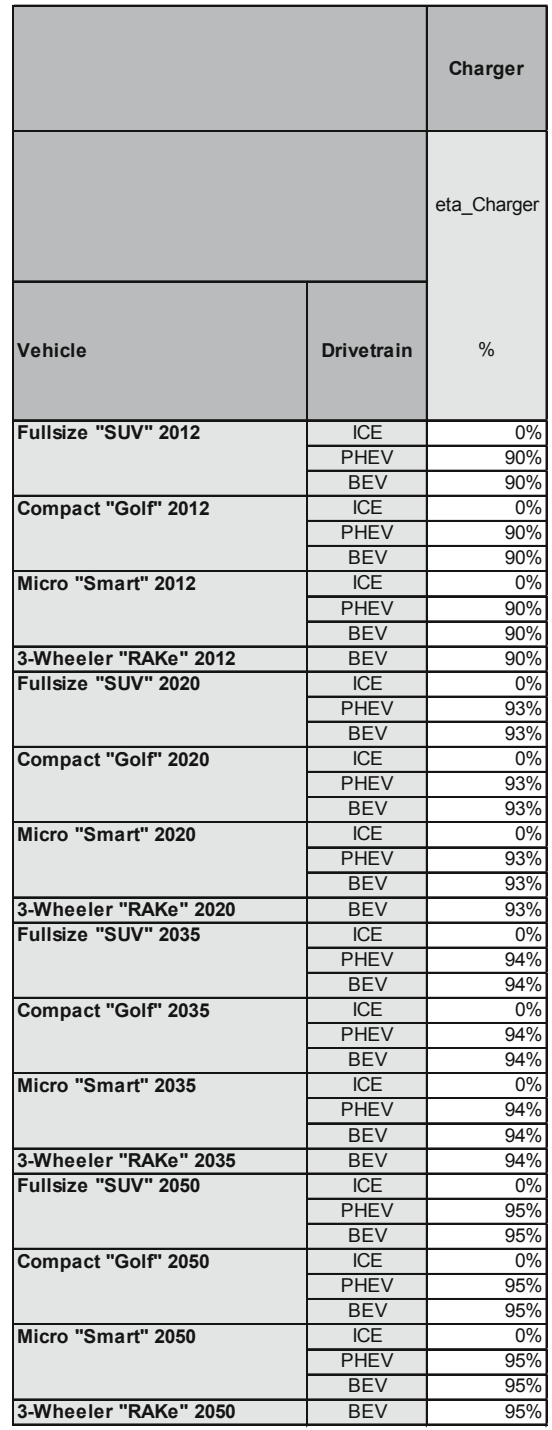

Tabelle 37: $\quad$ Annahmen zur Effizienz der Ladegeräte. 


\begin{tabular}{|c|c|c|c|c|c|c|c|}
\hline & & \multicolumn{2}{|c|}{$\begin{array}{l}\text { Traction: Real-world } \\
\text { energy demand }\end{array}$} & \multirow{2}{*}{\begin{tabular}{|c|}
\multicolumn{1}{c|}{$\begin{array}{c}\text { Traction } \\
\text { @plug }\end{array}$} \\
Plug level: \\
EVs and \\
PHEVs \\
kWh/100km
\end{tabular}} & \multicolumn{2}{|c|}{$\begin{array}{c}\text { Auxiliaries: Real-world } \\
\text { energy demand }\end{array}$} & \multirow{2}{*}{$\begin{array}{c}\begin{array}{c}\text { Auxiliaries } \\
\text { @plug }\end{array} \\
\\
\text { Plug level: } \\
\text { EVs and } \\
\text { PHEVs } \\
\text { kWh/100km }\end{array}$} \\
\hline & & $\begin{array}{c}\text { ICE: I/100km } \\
\text { gasoline }\end{array}$ & $\begin{array}{c}\text { EVs and } \\
\text { PHEVs } \\
\mathrm{kWh} / 100 \mathrm{~km}\end{array}$ & & $\begin{array}{c}\text { ICE: } 1 / 100 \mathrm{~km} \\
\text { gasoline }\end{array}$ & $\begin{array}{c}\text { EVs and } \\
\text { PHEVs } \\
\mathrm{kWh} / 100 \mathrm{~km}\end{array}$ & \\
\hline Vehicle & Drivetrain & l/100km & $\mathrm{kWh} / 100 \mathrm{~km}$ & $\begin{array}{c}\text { kWh/100km } \\
@ \text { plug }\end{array}$ & $\begin{array}{c}\text { (avg.eta_ICE } \\
\text {, eta_gen } \\
0.8)\end{array}$ & $\begin{array}{l}\mathrm{kWh} / 100 \mathrm{~km} \\
\text { from battery }\end{array}$ & $\begin{array}{l}\text { kWh/100km } \\
\text { @plug }\end{array}$ \\
\hline \multirow[t]{3}{*}{ Fullsize "SUV" 2012} & ICE & 10.0 & & & 0.96 & & \\
\hline & PHEV & 3.6 & 15.9 & 17.6 & & 4.5 & 5.0 \\
\hline & BEV & & 26.6 & 29.6 & & 4.5 & 5.0 \\
\hline \multirow[t]{3}{*}{ Compact "Golf" 2012} & ICE & 6.6 & & & 0.96 & & \\
\hline & PHEV & 2.3 & 10.4 & 11.5 & & 4.5 & 5.0 \\
\hline & BEV & & 17.4 & 19.3 & & 4.5 & 5.0 \\
\hline \multirow[t]{3}{*}{ Micro "Smart" 2012} & ICE & 5.2 & & & 0.90 & & \\
\hline & PHEV & 1.9 & 8.6 & 9.5 & & 3.4 & 3.8 \\
\hline & BEV & & 14.1 & 15.7 & & 3.4 & 3.8 \\
\hline 3-Wheeler "RAKe" 2012 & BEV & & 6.1 & 6.8 & & 2.4 & 2.6 \\
\hline \multirow[t]{3}{*}{ Fullsize "SUV" 2020} & ICE & 7.9 & & & 0.81 & & \\
\hline & PHEV & 1.8 & 16.8 & 18.0 & & 2.9 & 3.1 \\
\hline & BEV & & 22.6 & 24.3 & & 2.9 & 3.1 \\
\hline \multirow[t]{3}{*}{ Compact "Golf" 2020} & ICE & 5.1 & & & 0.81 & & \\
\hline & PHEV & 1.2 & 10.9 & 11.7 & & 2.9 & 3.1 \\
\hline & BEV & & 14.8 & 16.0 & & 2.9 & 3.1 \\
\hline \multirow[t]{3}{*}{ Micro "Smart" 2020} & ICE & 4.2 & & & 0.76 & & \\
\hline & PHEV & 1.0 & 9.1 & 9.8 & & 2.3 & 2.5 \\
\hline & BEV & & 12.1 & 13.0 & & 2.3 & 2.5 \\
\hline 3-Wheeler "RAKe" 2020 & BEV & & 5.2 & 5.6 & & 1.6 & 1.7 \\
\hline \multirow[t]{3}{*}{ Fullsize "SUV" 2035} & ICE & 6.3 & & & 0.70 & & \\
\hline & PHEV & 0.9 & 16.2 & 17.2 & & 2.3 & 2.5 \\
\hline & BEV & & 19.3 & 20.5 & & 2.3 & 2.5 \\
\hline \multirow[t]{3}{*}{ Compact "Golf" 2035} & ICE & 4.1 & & & 0.70 & & \\
\hline & PHEV & 0.6 & 10.6 & 11.3 & & 2.3 & 2.5 \\
\hline & BEV & & 12.9 & 13.7 & & 2.3 & 2.5 \\
\hline \multirow[t]{3}{*}{ Micro "Smart" 2035} & ICE & 3.4 & & & 0.66 & & \\
\hline & PHEV & 0.5 & 9.0 & 9.5 & & 1.9 & 2.1 \\
\hline & BEV & & 10.5 & 11.2 & & 1.9 & 2.1 \\
\hline 3-Wheeler "RAKe" 2035 & BEV & & 4.4 & 4.7 & & 1.3 & 1.3 \\
\hline \multirow[t]{3}{*}{ Fullsize "SUV" 2050} & ICE & 5.3 & & & 0.64 & & \\
\hline & PHEV & 0.5 & 15.2 & 16.0 & & 2.3 & 2.5 \\
\hline & BEV & & 17.1 & 18.0 & & 2.3 & 2.5 \\
\hline \multirow[t]{3}{*}{ Compact "Golf" 2050} & ICE & 3.5 & & & 0.64 & & \\
\hline & PHEV & 0.3 & 10.0 & 10.5 & & 2.3 & 2.5 \\
\hline & BEV & & 11.4 & 12.0 & & 2.3 & 2.5 \\
\hline \multirow[t]{3}{*}{ Micro "Smart" 2050} & ICE & 2.9 & & & 0.61 & & \\
\hline & PHEV & 0.3 & 8.4 & 8.9 & & 1.9 & 2.0 \\
\hline & BEV & & 9.4 & 9.9 & & 1.9 & 2.0 \\
\hline 3-Wheeler "RAKe" 2050 & BEV & & 3.9 & 4.1 & & 1.3 & 1.3 \\
\hline
\end{tabular}

Tabelle 38: $\quad$ Geschätzter Energiebedarf zur Fortbewegung in einer durchschnittlichen realen Fahrsituation und Energiebedarf für Zusatzverbraucher. 


\begin{tabular}{|c|c|c|c|c|c|c|c|c|c|c|}
\hline & & \multicolumn{9}{|c|}{ Auxiliaries } \\
\hline & & $\begin{array}{l}\text { demand: } \\
\text { light, wipers, } \\
\text { ventilation, } \\
\text { radio, navig. }\end{array}$ & $\begin{array}{l}\text { provision: } \\
\text { light, wipers, } \\
\text { ventilation, } \\
\text { radio, navig. }\end{array}$ & $\begin{array}{l}\text { demand: } \\
\text { heating } \\
\text { thermal }\end{array}$ & \begin{tabular}{|l} 
Heat pump \\
COP \\
(Jahresarbeit \\
szahl)
\end{tabular} & $\begin{array}{l}\text { provision: } \\
\text { heating } \\
\text { electric or } \\
\text { mechanical }\end{array}$ & $\begin{array}{l}\text { demand: } \\
\text { cooling } \\
\text { thermal }\end{array}$ & \begin{tabular}{|l} 
Heat pump \\
COP \\
(Jahresarbeit \\
szahl)
\end{tabular} & $\begin{array}{l}\text { provision: } \\
\text { cooling } \\
\text { electric or } \\
\text { mechanical }\end{array}$ & total \\
\hline Vehicle & Drivetrain & $\begin{array}{l}\text { kWh_el/100k } \\
\mathrm{m} \\
\text { electric }\end{array}$ & $\begin{array}{l}\mathrm{kWh} / 100 \mathrm{~km} \\
\text { (mech.,electri } \\
\text { c) }\end{array}$ & $\begin{array}{l}\text { kWh_th/100k } \\
m \text { thermal }\end{array}$ & $\begin{array}{l}\text { COP } \\
{[\text { W_th/W_el }]}\end{array}$ & \begin{tabular}{|l|}
$\mathrm{kWhl} / 100 \mathrm{~km}$ \\
(mech, \\
resistor, heat \\
pump)
\end{tabular} & $\begin{array}{l}\mathrm{kWh} \text { _th/100k } \\
\mathrm{m} \\
\text { heat pump }\end{array}$ & $\begin{array}{l}\text { COP } \\
{[\text { W_th/W_el] }}\end{array}$ & $\begin{array}{l}\mathrm{kWhl} / 100 \mathrm{~km} \\
\text { (mech, heat } \\
\text { pump) }\end{array}$ & $\begin{array}{l}\text { kWh/100km } \\
\text { (mech. } \\
\text { elect.) }\end{array}$ \\
\hline \multirow[t]{3}{*}{ Fullsize "SUV" 2012} & ICE & 1.00 & 1.00 & 3.00 & 1.00 & 0.00 & 1.00 & 2.00 & 0.50 & 1.50 \\
\hline & PHEV & 1.00 & 1.00 & 3.00 & 1.00 & 3.00 & 1.00 & 2.00 & 0.50 & 4.50 \\
\hline & BEV & 1.00 & 1.00 & 3.00 & 1.00 & 3.00 & 1.00 & 2.00 & 0.50 & 4.50 \\
\hline \multirow[t]{3}{*}{ Compact "Golf" 2012} & ICE & 1.00 & 1.00 & 3.00 & 1.00 & 0.00 & 1.00 & 2.00 & 0.50 & 1.50 \\
\hline & PHEV & 1.00 & 1.00 & 3.00 & 1.00 & 3.00 & 1.00 & 2.00 & 0.50 & 4.50 \\
\hline & BEV & 1.00 & 1.00 & 3.00 & 1.00 & 3.00 & 1.00 & 2.00 & 0.50 & 4.50 \\
\hline \multirow[t]{3}{*}{ Micro "Smart" 2012} & ICE & 1.00 & 1.00 & 2.00 & 1.00 & 0.00 & 0.80 & 2.00 & 0.40 & 1.40 \\
\hline & PHEV & 1.00 & 1.00 & 2.00 & 1.00 & 2.00 & 0.80 & 2.00 & 0.40 & 3.40 \\
\hline & BEV & 1.00 & 1.00 & 2.00 & 1.00 & 2.00 & 0.80 & 2.00 & 0.40 & 3.40 \\
\hline 3-Wheeler "RAKe" 2012 & BEV & 0.60 & 0.60 & 1.50 & 1.00 & 1.50 & 0.50 & 2.00 & 0.25 & 2.35 \\
\hline \multirow{3}{*}{ Fullsize "SUV" 2020} & ICE & 1.00 & 1.00 & 3.00 & 2.00 & 0.00 & 1.00 & 2.50 & 0.40 & 1.40 \\
\hline & PHEV & 1.00 & 1.00 & 3.00 & 2.00 & 1.50 & 1.00 & 2.50 & 0.40 & 2.90 \\
\hline & BEV & 1.00 & 1.00 & 3.00 & 2.00 & 1.50 & 1.00 & 2.50 & 0.40 & 2.90 \\
\hline \multirow[t]{3}{*}{ Compact "Golf" 2020} & ICE & 1.00 & 1.00 & 3.00 & 2.00 & 0.00 & 1.00 & 2.50 & 0.40 & 1.40 \\
\hline & PHEV & 1.00 & 1.00 & 3.00 & 2.00 & 1.50 & 1.00 & 2.50 & 0.40 & 2.90 \\
\hline & BEV & 1.00 & 1.00 & 3.00 & 2.00 & 1.50 & 1.00 & 2.50 & 0.40 & 2.90 \\
\hline \multirow[t]{3}{*}{ Micro "Smart" 2020} & ICE & 1.00 & 1.00 & 2.00 & 2.00 & 0.00 & 0.80 & 2.50 & 0.32 & 1.32 \\
\hline & PHEV & 1.00 & 1.00 & 2.00 & 2.00 & 1.00 & 0.80 & 2.50 & 0.32 & 2.32 \\
\hline & BEV & 1.00 & 1.00 & 2.00 & 2.00 & 1.00 & 0.80 & 2.50 & 0.32 & 2.32 \\
\hline 3-Wheeler "RAKe" 2020 & BEV & 0.60 & 0.60 & 1.50 & 2.00 & 0.75 & 0.50 & 2.50 & 0.20 & 1.55 \\
\hline \multirow[t]{3}{*}{ Fullsize "SUV" 2035} & ICE & 1.00 & 1.00 & 3.00 & 3.00 & 0.00 & 1.00 & 3.00 & 0.33 & 1.33 \\
\hline & PHEV & 1.00 & 1.00 & 3.00 & 3.00 & 1.00 & 1.00 & 3.00 & 0.33 & 2.33 \\
\hline & BEV & 1.00 & 1.00 & 3.00 & 3.00 & 1.00 & 1.00 & 3.00 & 0.33 & 2.33 \\
\hline \multirow[t]{3}{*}{ Compact "Golf" 2035} & ICE & 1.00 & 1.00 & 3.00 & 3.00 & 0.00 & 1.00 & 3.00 & 0.33 & 1.33 \\
\hline & PHEV & 1.00 & 1.00 & 3.00 & 3.00 & 1.00 & 1.00 & 3.00 & 0.33 & 2.33 \\
\hline & BEV & 1.00 & 1.00 & 3.00 & 3.00 & 1.00 & 1.00 & 3.00 & 0.33 & 2.33 \\
\hline \multirow[t]{3}{*}{ Micro "Smart" 2035} & ICE & 1.00 & 1.00 & 2.00 & 3.00 & 0.00 & 0.80 & 3.00 & 0.27 & 1.27 \\
\hline & PHEV & 1.00 & 1.00 & 2.00 & 3.00 & 0.67 & 0.80 & 3.00 & 0.27 & 1.93 \\
\hline & BEV & 1.00 & 1.00 & 2.00 & 3.00 & 0.67 & 0.80 & 3.00 & 0.27 & 1.93 \\
\hline 3-Wheeler "RAKe" 2035 & BEV & 0.60 & 0.60 & 1.50 & 3.00 & 0.50 & 0.50 & 3.00 & 0.17 & 1.27 \\
\hline \multirow{3}{*}{ Fullsize "SUV" 2050} & ICE & 1.00 & 1.00 & 3.00 & 3.00 & 0.00 & 1.00 & 3.00 & 0.33 & 1.33 \\
\hline & PHEV & 1.00 & 1.00 & 3.00 & 3.00 & 1.00 & 1.00 & 3.00 & 0.33 & 2.33 \\
\hline & BEV & 1.00 & 1.00 & 3.00 & 3.00 & 1.00 & 1.00 & 3.00 & 0.33 & 2.33 \\
\hline \multirow[t]{3}{*}{ Compact "Golf" 2050} & ICE & 1.00 & 1.00 & 3.00 & 3.00 & 0.00 & 1.00 & 3.00 & 0.33 & 1.33 \\
\hline & PHEV & 1.00 & 1.00 & 3.00 & 3.00 & 1.00 & 1.00 & 3.00 & 0.33 & 2.33 \\
\hline & BEV & 1.00 & 1.00 & 3.00 & 3.00 & 1.00 & 1.00 & 3.00 & 0.33 & 2.33 \\
\hline \multirow[t]{3}{*}{ Micro "Smart" 2050} & ICE & 1.00 & 1.00 & 2.00 & 3.00 & 0.00 & 0.80 & 3.00 & 0.27 & 1.27 \\
\hline & PHEV & 1.00 & 1.00 & 2.00 & 3.00 & 0.67 & 0.80 & 3.00 & 0.27 & 1.93 \\
\hline & BEV & 1.00 & 1.00 & 2.00 & 3.00 & 0.67 & 0.80 & 3.00 & 0.27 & 1.93 \\
\hline 3-Wheeler "RAKe" 2050 & BEV & 0.60 & 0.60 & 1.50 & 3.00 & 0.50 & 0.50 & 3.00 & 0.17 & 1.27 \\
\hline
\end{tabular}
Tabelle 39: Annahmen zum Energiebedarf von Sicherheits- und Komfort-
systemen. 


\begin{tabular}{|c|c|c|c|c|c|c|c|}
\hline & & \multicolumn{3}{|c|}{$\begin{array}{l}\text { TOTAL: Real-world energy demand } \\
\text { of vehicle and @plug }\end{array}$} & \multicolumn{3}{|c|}{ Pure electric mode } \\
\hline & & $\begin{array}{c}\text { Vehicle level: } \\
\text { ICE: I/100km } \\
\text { gasoline }\end{array}$ & $\begin{array}{c}\text { Vehicle level: } \\
\text { EVs and } \\
\text { PHEVs }\end{array}$ & $\begin{array}{l}\text { Plug level } \\
\text { incl. charge } \\
\text { losses for } \\
\text { EVs and } \\
\text { PHEVs }\end{array}$ & $\begin{array}{l}\text { Fraction of } \\
\text { plug-in } \\
\text { operation }\end{array}$ & $\begin{array}{c}\text { EVs and } \\
\text { PHEVs in } \\
\text { pure electric } \\
\text { mode }\end{array}$ & $\begin{array}{l}\text { Electric drive } \\
\text { autonomy }\end{array}$ \\
\hline Vehicle & Drivetrain & l/100km & $\mathrm{kWh} / 100 \mathrm{~km}$ & $\begin{array}{c}\text { kWh/100km } \\
\text { @ plug }\end{array}$ & $\begin{array}{c}\%(\mathrm{el} . \mathrm{km} \text { per } \\
\text { total } \mathrm{km})\end{array}$ & $\mathrm{kWh} / 100 \mathrm{~km}$ & $\mathrm{~km}$ \\
\hline \multirow[t]{3}{*}{ Fullsize "SUV" 2012} & ICE & 10.9 & & & $0 \%$ & & \\
\hline & PHEV & 3.6 & 20.4 & 22.6 & $60 \%$ & 30.9 & 48 \\
\hline & BEV & & 31.1 & 34.6 & $100 \%$ & 31.1 & 128 \\
\hline \multirow[t]{3}{*}{ Compact "Golf" 2012} & ICE & 7.5 & & & $0 \%$ & & \\
\hline & PHEV & 2.3 & 14.9 & 16.5 & $60 \%$ & 21.8 & 46 \\
\hline & BEV & & 21.9 & 24.3 & $100 \%$ & 21.9 & 137 \\
\hline \multirow[t]{3}{*}{ Micro "Smart" 2012} & ICE & 6.1 & & & $0 \%$ & & \\
\hline & PHEV & 1.9 & 12.0 & 13.3 & $60 \%$ & 17.7 & 45 \\
\hline & BEV & & 17.5 & 19.5 & $100 \%$ & 17.5 & 114 \\
\hline 3-Wheeler "RAKe" 2012 & BEV & & 8.5 & 9.4 & $100 \%$ & 8.5 & 94 \\
\hline \multirow[t]{3}{*}{ Fullsize "SUV" 2020} & ICE & 8.7 & & & $0 \%$ & & \\
\hline & PHEV & 1.8 & 19.7 & 21.2 & $75 \%$ & 25.3 & 91 \\
\hline & BEV & & 25.5 & 27.4 & $100 \%$ & 25.5 & 241 \\
\hline \multirow{3}{*}{ Compact "Golf" 2020} & ICE & 6.0 & & & $0 \%$ & & \\
\hline & PHEV & 1.2 & 13.8 & 14.8 & $75 \%$ & 17.4 & 88 \\
\hline & BEV & & 17.7 & 19.1 & $100 \%$ & 17.7 & 260 \\
\hline \multirow[t]{3}{*}{ Micro "Smart" 2020} & ICE & 4.9 & & & $0 \%$ & & \\
\hline & PHEV & 1.0 & 11.5 & 12.3 & $75 \%$ & 14.5 & 85 \\
\hline & BEV & & 14.4 & 15.5 & $100 \%$ & 14.4 & 214 \\
\hline 3-Wheeler "RAKe" 2020 & BEV & & 6.7 & 7.2 & $100 \%$ & 6.7 & 183 \\
\hline \multirow[t]{3}{*}{ Fullsize "SUV" 2035} & ICE & 7.0 & & & $0 \%$ & & \\
\hline & PHEV & 0.9 & 18.5 & 19.7 & $85 \%$ & 21.4 & 220 \\
\hline & BEV & & 21.6 & 23.0 & $100 \%$ & 21.6 & 579 \\
\hline \multirow[t]{3}{*}{ Compact "Golf" 2035} & ICE & 4.8 & & & $0 \%$ & & \\
\hline & PHEV & 0.6 & 13.0 & 13.8 & $85 \%$ & 14.9 & 211 \\
\hline & BEV & & 15.2 & 16.2 & $100 \%$ & 15.2 & 618 \\
\hline \multirow[t]{3}{*}{ Micro "Smart" 2035} & ICE & 4.0 & & & $0 \%$ & & \\
\hline & PHEV & 0.5 & 10.9 & 11.6 & $85 \%$ & 12.5 & 200 \\
\hline & BEV & & 12.5 & 13.2 & $100 \%$ & 12.5 & 503 \\
\hline 3-Wheeler "RAKe" 2035 & BEV & & 5.7 & 6.1 & $100 \%$ & 5.7 & 440 \\
\hline \multirow[t]{3}{*}{ Fullsize "SUV" 2050} & ICE & 5.9 & & & $0 \%$ & & \\
\hline & PHEV & 0.5 & 17.5 & 18.4 & $90 \%$ & 19.2 & 281 \\
\hline & BEV & & 19.4 & 20.4 & $100 \%$ & 19.4 & 741 \\
\hline \multirow[t]{3}{*}{ Compact "Golf" 2050} & ICE & 4.1 & & & $0 \%$ & & \\
\hline & PHEV & 0.3 & 12.3 & 13.0 & $90 \%$ & 13.5 & 268 \\
\hline & BEV & & 13.8 & 14.5 & $100 \%$ & 13.8 & 784 \\
\hline \multirow[t]{3}{*}{ Micro "Smart" 2050} & ICE & 3.5 & & & $0 \%$ & & \\
\hline & PHEV & 0.3 & 10.4 & 10.9 & $90 \%$ & 11.3 & 255 \\
\hline & BEV & & 11.3 & 11.9 & $100 \%$ & 11.3 & 637 \\
\hline 3-Wheeler "RAKe" 2050 & BEV & & 5.2 & 5.5 & $100 \%$ & 5.2 & 555 \\
\hline
\end{tabular}

Tabelle 40: $\quad$ Totaler Energiebedarf (ab Zapfsäule bzw. AC-Steckdose) sowie totale rein elektrische Reichweite (bei hundertprozentiger Ausnutzung der Batteriekapazität). 


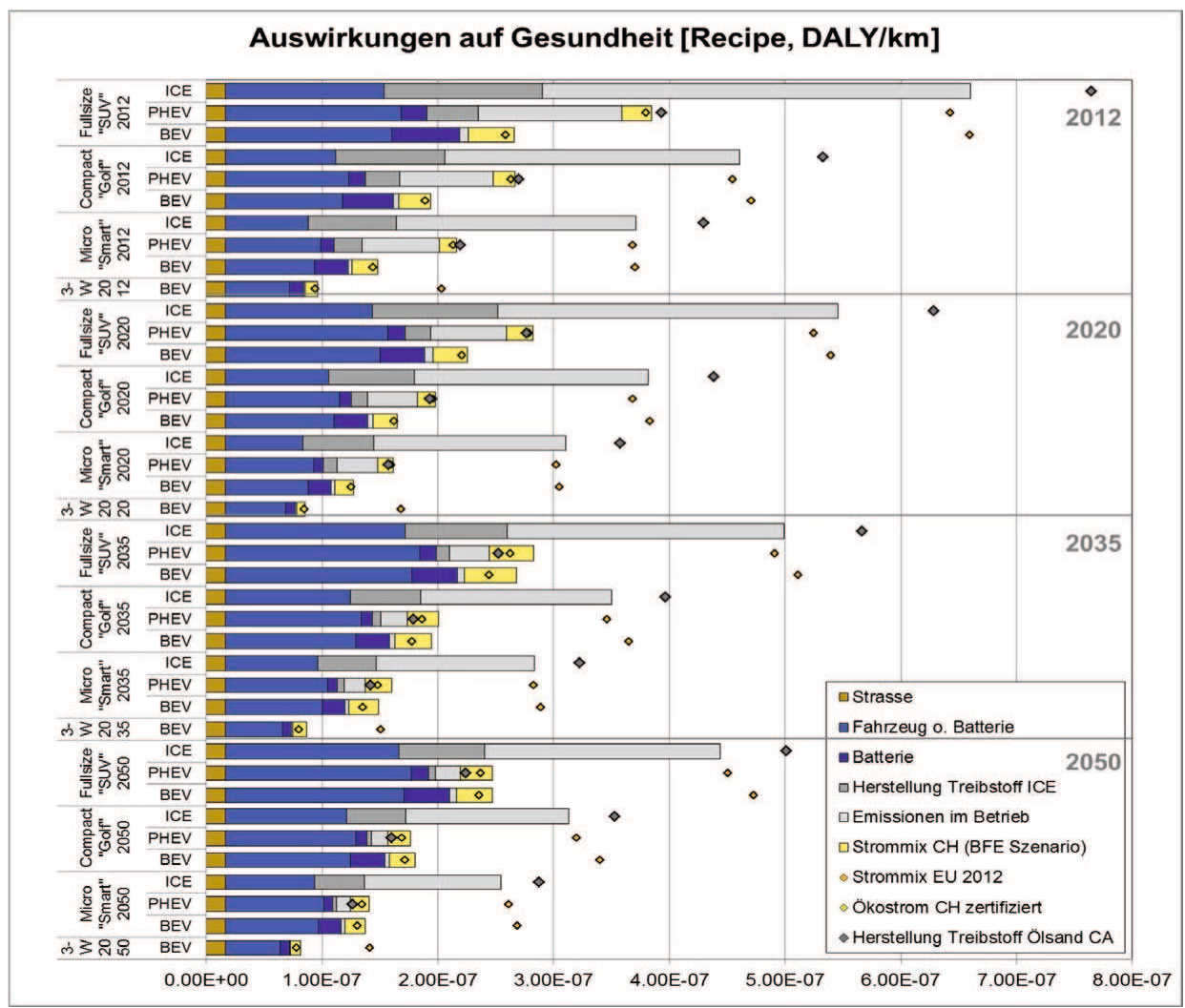

Abbildung 55: Lebenszyklus Human Health Impact (Recipe) für die drei untersuchten Antriebsstränge (ICE, PHEV, BEV) und die vier Zeitpunkte (2012/20/35/50) für die vier Fahrzeugklassen (Fullsize, Compact, Micro, 3-Wheeler). Die Punkte bezeichnen jeweils die Veränderung bei Anpassung der Benzinqualität (bei ICE, Benzin aus kanadischen Ölsanden) und bei Veränderung des Strommixes für PHEV/BEV-Fahrzeuge (europäischer Strommix bzw. zertifizierter $\mathrm{CH}$-Ökostrom). 


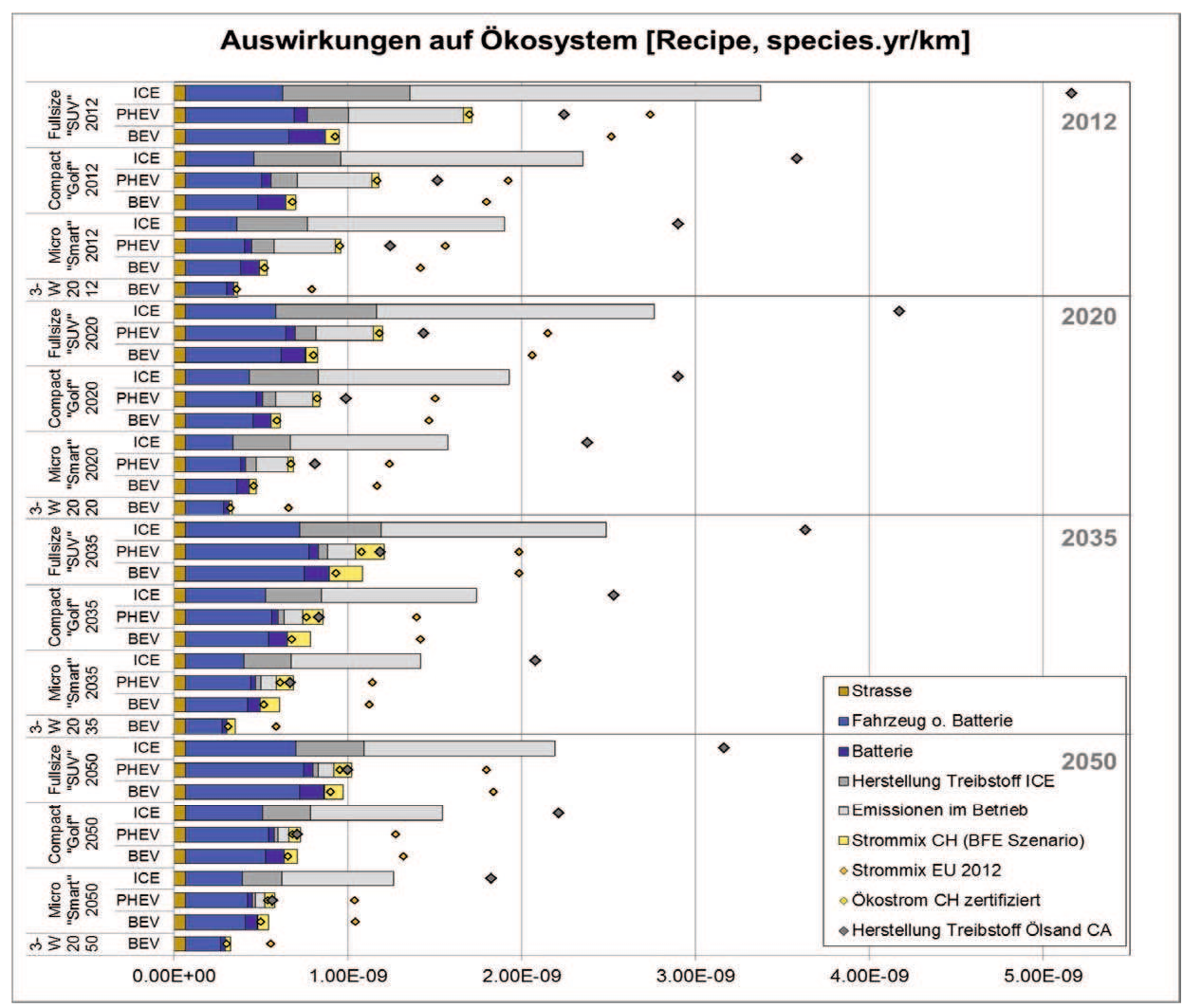

Abbildung 56: Lebenszyklus Ecosystems Impact (Recipe) für die drei untersuchten Antriebsstränge (ICE, PHEV, BEV) und die vier Zeitpunkte (2012/20/35/50) für die vier Fahrzeugklassen (Fullsize, Compact, Micro, 3-Wheeler). Die Punkte bezeichnen jeweils die Veränderung bei Anpassung der Benzinqualität (bei ICE, Benzin aus kanadischen Ölsanden) und bei Veränderung des Strommixes für PHEV/BEV-Fahrzeuge (europäischer Strommix bzw. zertifizierter $\mathrm{CH}$-Ökostrom). 


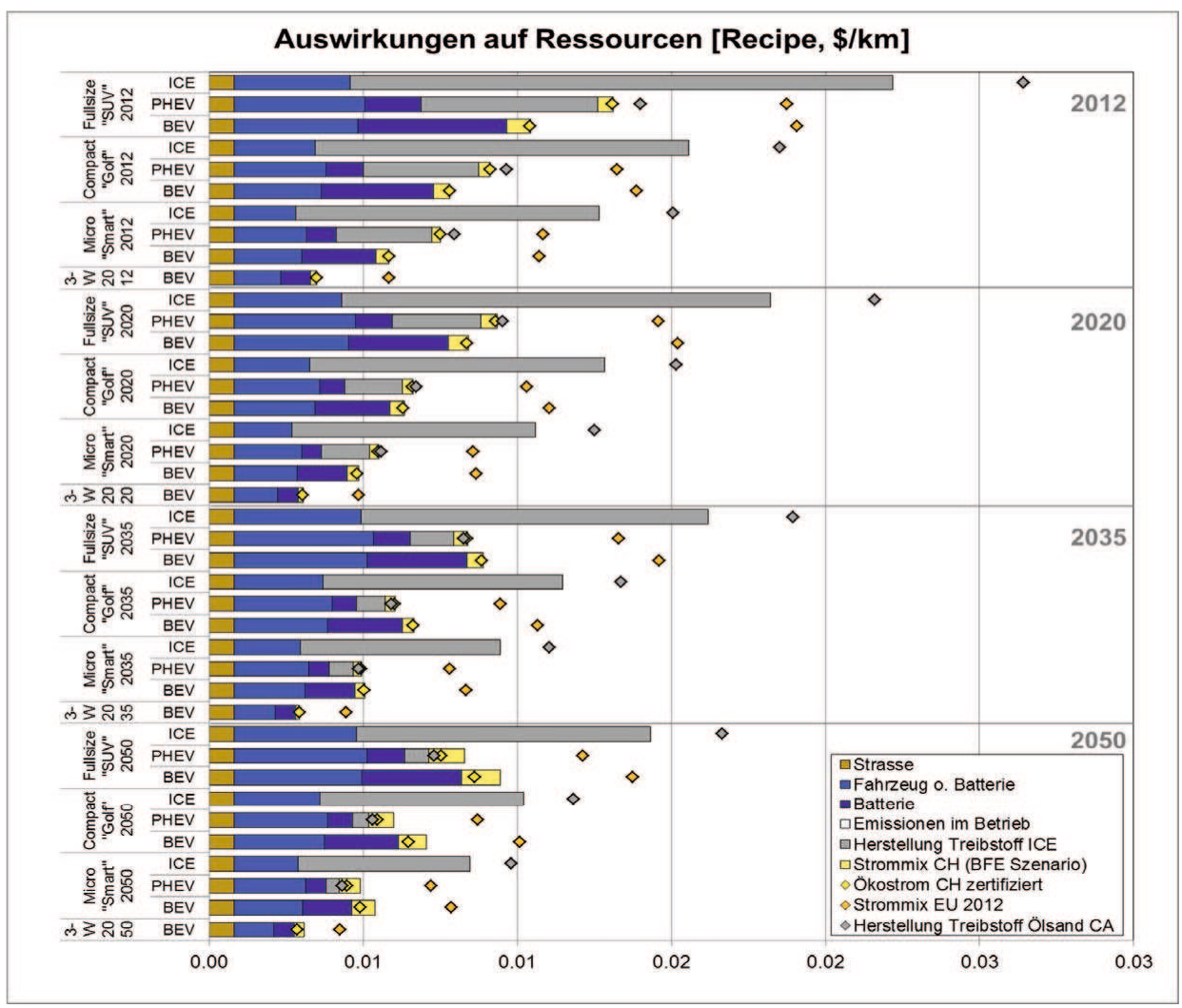

Abbildung 57: Lebenszyklus Resources Impact (Recipe) für die drei untersuchten Antriebsstränge (ICE, PHEV, BEV) und die vier Zeitpunkte (2012/20/35/50) für die vier Fahrzeugklassen (Fullsize, Compact, Micro, 3-Wheeler). Die Punkte bezeichnen jeweils die Veränderung bei Anpassung der Benzinqualität (bei ICE, Benzin aus kanadischen Ölsanden) und bei Veränderung des Strommixes für PHEV/BEV-Fahrzeuge (europäischer Strommix bzw. zertifizierter $\mathrm{CH}$-Ökostrom). 


\section{Verzeichnis der Projektbeteiligten}

Projektleitung TA-SWISS:

Lucienne Rey, TA-SWISS

Projektgruppe:

Rainer Zah, Empa, Dübendorf, Projektleiter Empa

Hans-Jörg Althaus, Empa, Dübendorf

Marcel Gauch, Empa, Dübendorf

Patrick Wäger, Empa, Dübendorf

Rolf Widmer, Empa, Dübendorf

Peter de Haan, Ernst Basler + Partner AG, Zollikon, Projektleiter EBP

Katrin Bernath, Ernst Basler + Partner AG, Zollikon

Frank Bruns, Ernst Basler + Partner AG, Zollikon

Denise Fussen, Ernst Basler + Partner AG, Zollikon 


\section{Verzeichnis der Begleitgruppenmitglieder}

Ruedi Jörg-Fromm, Zürich, TA-SWISS Leitungsausschuss (Vorsitzender der Begleitgruppe)

Heidi Blattmann, Wissenschaftsjournalistin, Herrliberg, TA-SWISS Leitungsausschuss

Christian Bühlmann, Bundesamt für Energie BFE, Ittigen

Volker Fröse, Bundesamt für Strassen ASTRA, Bern

Lino Guzzella, ETH Zürich, Zürich

Stefan Hirschberg, Paul Scherrer Institut PSI, Villigen

Kurt Hug, Berner Fachhochschule, Vaufflin

Christoph Jahn, Bundesamt für Strassen ASTRA, Bern

Mario Keller, INFRAS, Bern

Dieter Kraft, Bosch, Stuttgart

Cornelia Moser, Bundesamt für Strassen ASTRA, Bern

Tobias Ott, ETH Zürich, Zürich

Felix Reutimann, Bundesamt für Umwelt BAFU, Bern

Martin Schiess, Bundesamt für Umwelt BAFU, Bern

Fridolin Stähli, Fachhochschule Nordwestschweiz, Windisch

Daniel Wachter, Bundesamt für Raumentwicklung ARE, Bern

Michael Weber, Bundesamt für Umwelt BAFU, Bern 


\section{Weitere Publikationen von TA-SWISS}

Rainer Zah, Claudia Binder, Stefan Bringezu, Jürgen Reinhard, Alfons Schmid, Helmut Schütz

\section{Future Perspectives of 2nd Generation Biofuels}

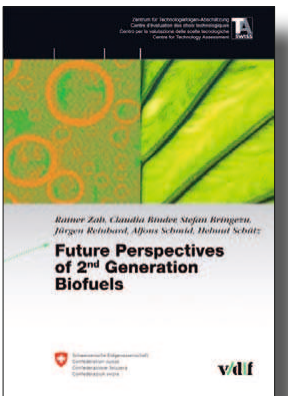

2010, 328 Seiten, zahlreiche Grafiken und Tabellen, durchg. farbig, Format $16 \times 23 \mathrm{~cm}$, broschiert ISBN 978-3-7281-3334-2 auch als eBook erhältlich

Lorenz Hilty, Britta Oertel, Michaela Wölk, Kurt Pärli

\section{Lokalisiert und identifiziert}

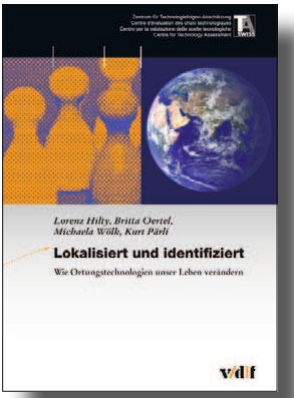

2012, 294 Seiten, zahlreiche Abbildungen und Grafiken, Format $16 \times 23 \mathrm{~cm}$, broschiert ISBN 978-3-7281-3460-8

auch als eBook (Open Access) erhältlich

\section{$\mathbf{v} / \mathbf{l} \mathbf{f}$}

Anne Eckhardt, Andreas Bachmann, Michèle Marti, Bernhard Rütsche, Harry Telser

\section{Human Enhancement}

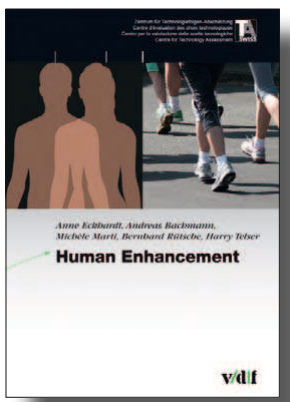

2011, 300 Seiten, zahlreiche Abbildungen und Tabellen, Format $16 \times 23 \mathrm{~cm}$, broschiert ISBN 978-3-7281-3396-0 auch als eBook (Open Access) erhältlich

Heidrun Becker, Mandy Scheermesser, Michael Früh, Yvonne Treusch, Holger Auerbach, Richard Alexander Hüppi, Flurina Meier

\section{Robotik in Betreuung und Gesundheitsversorgung}

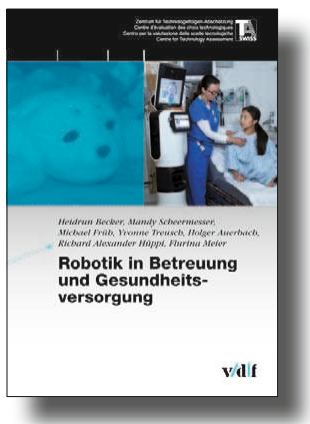

2013, 252 Seiten, zahlreiche Abbildungen, Format $16 \times 23 \mathrm{~cm}$, broschiert ISBN 978-3-7281-3520-9 auch als eBook (Open Access) erhältlich

vdf Hochschulverlag AG an der ETH Zürich, Voltastrasse 24, VOB D, CH-8092 Zürich Tel. +41 (0)44 63242 42, Fax +41 (0)44 63212 32, verlag@vdf.ethz.ch, www.vdf.ethz.ch 


\section{www.ta-swiss.ch}

— Ein Kompetenzzentrum der

Akademien der Wissenschaften Schweiz

Elektrofabrzeuge können einen Beitrag zu umweltfreundlicherem Verkehr leisten - Voraussetzung ist, dass sie mit $\mathrm{CO}_{2}$-armem Strom betrieben werden. Aber auch Fabrzeuge mit Verbrennungsmotor werden in Zukunft deutlich effizienter werden. Der $\mathrm{CO}_{2}$-Ausstoss der schweizerischen Autoflotte wird daher trotz Bevölkerungs- und Mobilitätswachstum zurückgehen. Dieser Trend zu weniger Energieverbrauch wird durch die Elektromobilität zusätzlich gefördert.

Vor allem kleinere und leichtere Fahrzeuge lassen sich gut elektrifizieren - dank Fortschritten in der Batterietechnik zunehmend ohne Kompromisse. Energieversorger und ÖV-Betreiber fördern den Trend, indem sie die Nutzung urbaner Kleinwagen und gemischter Mobilitätsformen erleichtern.

Mebr Elektromobilität führt zu geringeren Treibstoffzoll-Einnabmen, was Finanzierungslïcken bewirkt und den Wechsel hin zu einer fabrleistungs- und effizienzabbängigen Besteuerung beschleunigt. Sie reduziert auch die Abhängigkeit von Erdöl und erlaubt die Integration erneuerbarer, lokal erzeugter Elektrizität. Demgegenüber beschleunigt sich die Verlagerung der Umweltbelastung von der Strasse hin zu Herstellung und Entsorgung. Nachbaltige Elektromobilität erfordert daher auch die Ausweitung des energiepolitisches Sichtfeldes auf den ganzen Lebenszyklus und ein Schliessen der Ressourcen-Kreisläufe.

\section{$\mathbf{V} / \mathbf{l} \mathbf{f}$}

TA-SWISS 59/2013

ISBN 978-3-7281-3487-5

(Printausgabe)

Download open access:

ISBN 978-3-7281-3488-2

DOI 10.3218/3488-2
Schweizerische Eidgenossenschaft

Confédération suisse

Confederazione Svizzera

Confederaziun svizra
Bundesamt für Energie BFE Bundesamt für Strassen ASTRA Bundesamt für Umwelt BAFU 